\title{
SUZELLE BLAIS
}

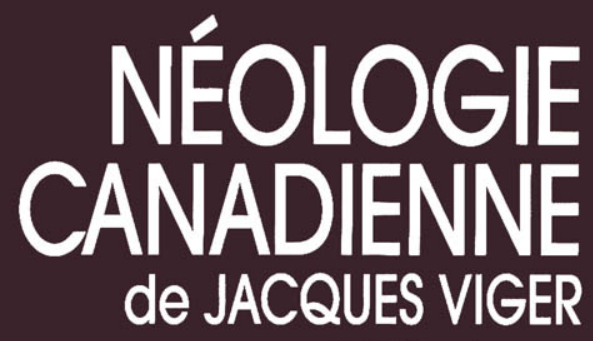

(MANUSCRIIS DE 1810)

\author{
Préface de \\ ANDRÉ LAPIERRE
}

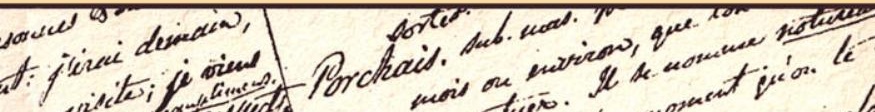

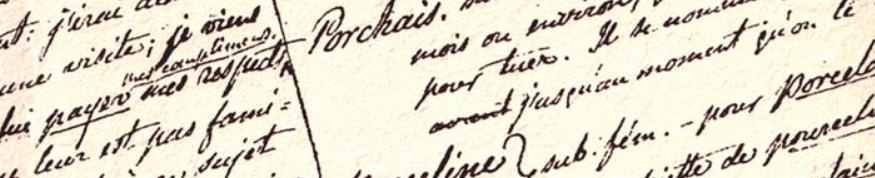

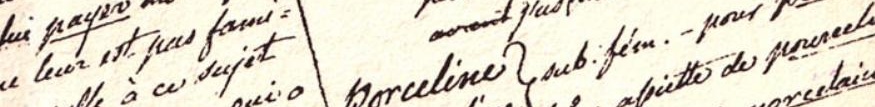

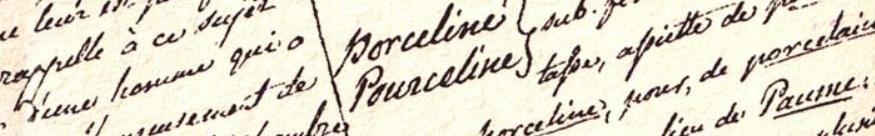

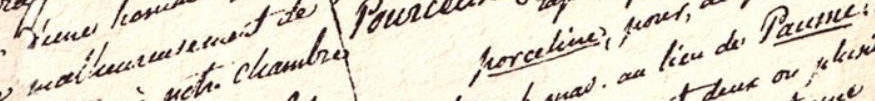

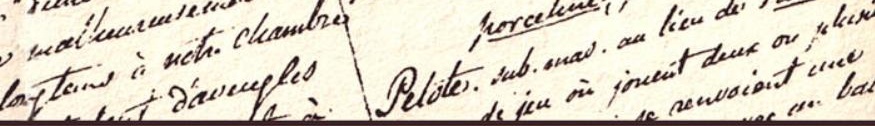

Collection AMÉRIQUE FRANÇAISE -
Les Presses

de l'Université

d'Oltawa 


\section{NÉOlogie CANADIENNE}




\title{
COLLECTION «AMÉRIQUE FRANÇAISE *
}

La collection «Amérique française» regroupe des ouvrages portant sur le fait français en Amérique, en particulier à l'extérieur du Québec, soit en Ontario, en Acadie, dans l'Ouest canadien et aux États-Unis. La collection, conforme à la philosophie de la maison d'édition, accueille des manuscrits de langues française et anglaise.

\author{
Directeur: $\quad$ André Lapierre \\ Comité éditorial : Roger Bernard \\ Chad Gaffiel
}

Déja parus :

Artisans de la modernité. Les centres culturels en Ontario français

Diane Farmer

2-7603-0427-2, 239 pages

Aux origines de l'identité franco-ontarienne. Éducation, culture, économie Chad Gaffield

2-7603-0255-5, 284 pages

Les Écrits de Pierre Potier

Robert Toupin

2-7603-0426-4, 1329 pages

Le Franģais des Canadiens à la veille de la Conquête.

Témoignage du père Pierre Pbilippe Potier, s.j.

Peter W. Halford

2-7603-0271-7, 380 pages 
SUZELLE BLAIS

\section{Néologie CANADIENNE,}

ou

\section{DICTIONNAIRE}

des mots créés en Canada

$\&$ maintenant en vogue;

- des mots dont la prononciation \& l'ortographe sont différentes de la prononciation

$\&$ ortographe françoises, quoique employés dans une acception semblable ou contraire; et des mots étrangers qui se sont glissés dans notre langue.

de

JACQUES VIGER (MANUSCRITS DE 1810)

ÉDITION AVEC ÉTUDE LINGUISTIQUE

Préface d'André Lapierre

Collection AMÉRIQUE FRANÇAISE

$$
N^{0} 5
$$

Les Presses de l'Université d'Ottawa 


\section{DONNÉES DE CATALOGAGE AVANT PUBLICATION (CANADA)}

Viger, J. (Jacques), 1787-1858

Néologie canadienne, ou Dictionnaire

(Collection Amérique française)

Texte intégral des deux versions manuscrites de Jacques Viger.

Comprend des références bibliographiques et un index.

ISBN 2-7603-0479-5

1. Français (Langue)-Canada-Idiotismes-Dictionnaires. 2. Français (Langue)Canada-Mots et locutions. 3. Français (Langue)-Néologismes-Dictionnaires. 4. Viger, J. (Jacques), 1787-1858. 5. Français (Langue)-Canada-Lexicographie. 6. Français (Langue)-19e siècle. I. Blais, Suzelle. II. Titre. III. Titre : Dictionnaire. IV. Collection.

Cet ouvrage a été publié grâce à une contribution de la Fédération canadienne des sciences humaines et sociales, dont les fonds proviennent du Conseil de recherches en sciences humaines du Canada.

Les Presses de l'Université d'Ottawa remercient le Conseil des Arts du Canada, le ministère du Patrimoine canadien et l'Université d'Ottawa de l'aide qu'ils apportent à leur programme de publication.

Maquette de la couverture : Robert Dolbec

Illustration de couverture : Extrait d'une page du manuscrit 1 de la Néologie canadienne de Jacques Viger. Manuscrit conservé au Musée de la civilisation, fonds d'archives du Séminaire de Québec.

Mise en pages : Danielle Péret

"Tous droits de traduction et d'adaptation, en totalité ou en partie, réservés pour tous les pays. La reproduction d'un extrait quelconque de ce livre, par quelque procédé que ce soit, tant électronique que mécanique, en particulier par photocopie et par microfilm, est interdite sans l'autorisation écrite de l'éditeur. »

ISBN 2-7603-0479-5

(C) Les Presses de l'Université d'Ottawa, 1998

542, King Edward, Ottawa (Ont.), Canada K1N 6N5

press@uottawa.ca http://www.uopress.uottawa.ca

Imprimé et relié au Canada 
Et comme les mots sont plus mystérieux que les faits, il me reste dans les oreilles des sons d'une intelligence secrète.

PterRe Mac OrLan, La petite cloche de Sorbonne

\section{REMERCIEMENTS}

J'adresse mes remerciements très sincères à André Lapierre, de l'Université d'Ottawa, qui m'a proposé de publier dans la collection "Amérique française" la Néologie canadienne de Jacques Viger. Grâce à ses encouragements et à la confiance qu'il m'a témoignée, cet ouvrage voit enfin le jour.

Je dois beaucoup à Ludmila Bovet, linguiste, qui a accepté avec enthousiasme de lire un premier état de mon texte. Ses commentaires linguistiques et lexicographiques judicieux et sa connaissance de l'histoire de la langue me furent des plus précieux et je tiens à lui exprimer toute ma reconnaissance.

Merci à Peter W. Halford, de l'Université de Windsor (Ontario), qui a eu l'amabilité de me faire profiter de son expérience d'éditeur, en relisant mon manuscrit et en me fournissant des renseignements importants. Qu'il soit assuré de ma profonde gratitude.

Je suis reconnaissante à ceux et celles qui, comme Madeleine Faucher, du Musée de la Civilisation, aux archives du Séminaire de Québec, m'ont donné accès aux documents manuscrits de Jacques Viger et qui ont facilité mes recherches. 
This page intentionally left blank 


\section{PRÉFACE}

La collection «Amérique française» accueillait en 1994 (Peter W. Halford : Le français des Canadiens à la veille de la Conquête) le manuscrit du jésuite Pierre-Philippe Potier, premier document lexicographique franco-canadien du milieu du XVIII ${ }^{\mathrm{e}}$ siècle. Il ne restait plus alors qu'un seul témoignage d'importance sur le français canadien qui ne fût publié dans sa totalité. Avec la parution du présent ouvrage, c'est maintenant chose faite. Suzelle Blais nous livre ici le texte in extenso du premier dictionnaire réalisé par un locuteur natif du pays canadien, Jacques Viger.

Le futur historien et maire de la ville de Montréal s'est vraisemblablement mis à la rédaction d'un dictionnaire du parler canadien à l'époque où, encore dans la jeune vingtaine, il était au journal Le Canadien à Québec. Peut-être en avait-il déjà conçu le projet au cours de ses études chez les Sulpiciens de Montréal, alors que son esprit curieux pouvait déjà noter les différences entre le parler de ses éducateurs européens et celui qu'il entendait tous les jours dans son entourage. Peut-être avait-il même commencé la rédaction de certains articles. Quoi qu'il en soit, le projet de publication est resté à l'état de manuscrit. Tout comme dans le cas du travail du père Potier, c'est encore une fois la Société du parler français au Canada qui a porté à la connaissance du grand public l'existence du dictionnaire de $V$ iger en publiant de larges extraits du manuscrit quelque cent ans plus tard dans son Bulletin.

Lédition du texte intégral de la Néologie canadienne de Viger constituait un défi particulier en raison de l'existence, en fait, de deux manuscrits représentant deux versions du même travail : le premier étant le premier jet, le second, une version soignée, revue et corrigée. En choisissant de publier l'ensemble des cahiers de l'œuvre, Suzelle Blais nous livre ici non seulement le texte fidèle du dictionnaire tel que Viger l'a conçu mais aussi, grâce à la comparaison des deux manuscrits, des indices précieux sur l'élaboration même de l'ouvrage.

Ce dictionnaire est le reflet de l'idéologie dominante dans la classe cultivée francophone du Bas-Canada au début du XIX siècle. On notera qu'il s'agit d'un dictionnaire différentiel, rédigé dans une perspective normative par rapport à l'usage européen de l'époque. Viger traduit une préoccupation de l'élite de son temps, celle du nécessaire alignement du 
français canadien sur la norme de Paris pour en assurer la défense et la promotion. Ce n'est que vers la fin du XIX ${ }^{\mathrm{e}}$ siècle, alors que se multiplient en Europe les glossaires des dialectes français, que les lexicographes canadiens seront en mesure de mieux apprécier les origines des particularismes que Viger affecte de l'appellatif néologies canadiennes.

Le travail de Viger mérite l'attention des spécialistes à plus d'un titre. En faisant abstraction du discours normatif qui caractérise l'ouvrage, on devinera aisément l'importance que revêt ce dictionnaire pour l'étude du lexique français en général et franco-canadien en particulier. On y trouve, en premier lieu, les attestations d'origine de quelque 400 lexies ou de modifications sémantiques de mots français en terre canadienne. En outre, les indications sur la distribution géographique de mots comme travail, menoirs, canard, et bombe intéresseront les dialectologues. Les historiens de la langue y trouveront des datations nouvelles, certaines surprenantes, comme l'adjectif loucbeur, dont la première attestation recule maintenant de plus de 125 ans. Les emprunts à l'anglais que note Viger révèlent que les processus classiques d'intégration lexicale étaient déjà à l'ouvre dès le début du $\mathrm{XIX}^{\mathrm{e}}$ siècle. Certains emprunts attestés chez Viger éclairent même nos connaissances sur le lexique anglais au Canada. Le mot dram, par exemple, déjà noté comme emprunt dans la terminologie du flottage du bois en 1810, ne sera attesté en lexicographie anglo-canadienne qu'en 1967. D'ailleurs, en prenant un peu de recul, on voit que c'est tout un volet de la société québécoise du début du XIX ${ }^{\mathrm{e}}$ siècle qui se profile à travers les mots et expressions que Viger a choisi de consigner dans son recueil. Dans ce sens, le travail de Suzelle Blais dépasse largement la linguistique et éclaire de façon inédite et originale l'histoire sociale du Canada français de la première moitié du siècle dernier.

Avec la publication des manuscrits de Viger, la collection «Amérique française» est fière de reconstituer aujourd'hui le maillon manquant de cette chaine précieuse de témoignages lexicographiques sur le français canadien du XIX ${ }^{\mathrm{e}}$ siècle. Il faut savoir gré à Suzelle Blais, par son patient labeur et son souci de perfection, d'avoir ainsi fourni à la communauté scientifique un ouvrage inestimable sur l'histoire de la langue française en même temps que sur l'élaboration du lexique français en terre canadienne.

André Lapierre

Département de linguistique Université d'Ottawa Directeur de la collection «Amérique française» 


\section{TABLE DES MATIÈRES}

Préface d'André Lapierre $\ldots \ldots \ldots \ldots \ldots \ldots \ldots \ldots$

Introduction $\ldots \ldots \ldots \ldots \ldots \ldots \ldots \ldots \ldots \ldots \ldots \ldots$

L'auteur ........................... 13

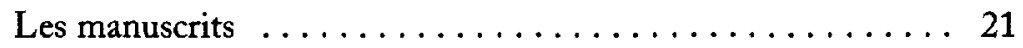

L'édition de la Société du parler français au Canada ....... 28

La présente édition $\ldots \ldots \ldots \ldots \ldots \ldots \ldots \ldots \ldots \ldots \ldots \ldots \ldots \ldots$

Établissement du texte $\ldots \ldots \ldots \ldots \ldots \ldots \ldots \ldots \ldots . \ldots 30$

Liste des termes, signes conventionnels et abréviations . . . . 33

Édition des manuscrits . . . . . . . . . . . . . . 37

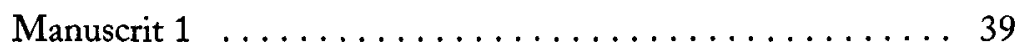

Notes du manuscrit $1 \ldots \ldots \ldots \ldots \ldots \ldots \ldots$

Manuscrit $2 \ldots \ldots \ldots \ldots \ldots \ldots \ldots \ldots \ldots$

Notes du manuscrit $2 \ldots \ldots \ldots \ldots \ldots \ldots \ldots \ldots \ldots$

Étude linguistique $\ldots \ldots \ldots \ldots \ldots \ldots \ldots \ldots \ldots \ldots \ldots \ldots$

Aspects phonétiques . . . . . . . . . . . . . 133

Vocalisme ........................ 133

Consonantisme ..................... 139

Agglutination . . . . . . . . . . . . . . . 144

Étymologie populaire $\ldots \ldots \ldots \ldots \ldots \ldots \ldots \ldots \ldots$

Aspects morphologiques et syntaxiques ............ 146

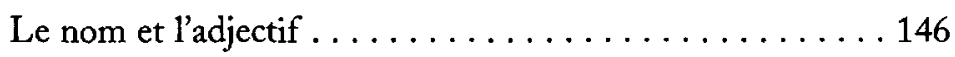

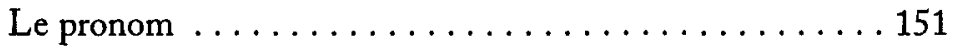

Le verbe .................... 151

L'adverbe $\ldots \ldots \ldots \ldots \ldots \ldots \ldots \ldots \ldots \ldots \ldots \ldots \ldots \ldots$

La préposition $\ldots \ldots \ldots \ldots \ldots \ldots \ldots \ldots \ldots \ldots \ldots$ 


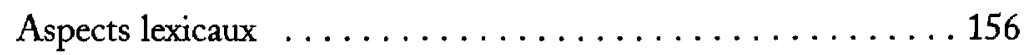

Archaïsmes, dialectalismes et innovations formelles

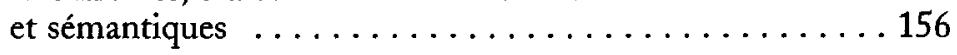

Emprunts aux langues amérindiennes $\ldots \ldots \ldots \ldots \ldots 232$

Emprunts à l'anglais . . . . . . . . . . . . 235

Datations .........................240

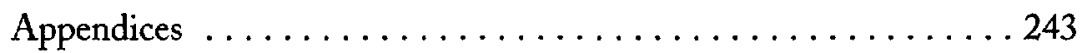

I. Listes des mots et des expressions relevés par Jacques Viger . . . . . . . . . . . . . . . . 245

II. Listes des mots et des expressions relevés par Ross Cuthbert . . . . . . . . . . . . . . . . . 252

Regroupement onomasiologique $\ldots \ldots \ldots \ldots \ldots \ldots \ldots . \ldots 267$

Les comparaisons $\ldots \ldots \ldots \ldots \ldots \ldots \ldots \ldots \ldots \ldots \ldots \ldots \ldots$

Bibliographie . . . . . . . . . . . . . . . . . 279

I. Manuscrits ....................... 279

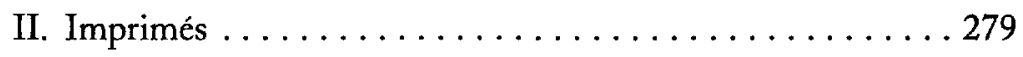

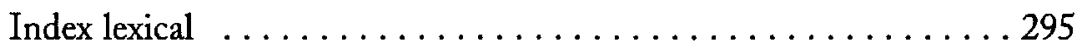




\section{INTRODUCTION}

\section{L'AUTEUR}

\section{NOTES BIOGRAPHIQUES}

Jacques Viger est né à Montréal le 7 mai 1787. Il est le fils de Jacques Viger et d'Amaranthe Prévost. Il eut pour parrain Joseph Papineau, notaire, député et père de Louis-Joseph Papineau, et pour marraine Marianne Cherrier. En 1799, Viger est inscrit au Collège Saint-Raphaël (fondé en 1773, il prendra en 1806 le nom de Collège de Montréal), dirigé par les Sulpiciens, où il reçoit une formation classique. C'est durant ses années d'études qu'il se lie d'amitié avec l'un de ses condisciples, Michel Bibaud, qui fera carrière comme professeur, journaliste et auteur et avec qui il restera en contact toute sa vie. Bibaud fera paraitre dans les revues qu'il fondera, telles que La Bibliothèque canadienne et L'Encyclopédie canadienne, de nombreux extraits d'ouvrages de Viger sur l'histoire du Canada dont La Saberdache et Les Tablettes.

Nommé rédacteur au journal Le Canadien, en 1808, Viger y restera de novembre 1808 à mai 1809. Fondé à Québec en 1806 par Pierre Bédard et François-Xavier Blanchet, Le Canadien s'intéresse principalement à la politique et à la défense des droits des Canadiens français et sert de tribune aux poètes et aux prosateurs.

Si l'on en juge par la lettre de félicitations que Viger a reçue d'un ami au moment de sa nomination, le poste n'était pas de tout repos et demandait beaucoup de jugement :

Je vous fais mon compliment de la place que vous venez d'avoir; - mais, mon jeune ami, permettez-moi de vous dire qu'elle est bien dangereuse, car il faut satisfaire le public (ou ses amis), et pourtant aussi ne pas offenser le gouvernement, qui a le bras long et fort. Il faut donc trouver un milieu, qui est quelquefois un peu difficile à trouver. (La Saberdache bleue, vol. 1, p. 51.)

C'est à cette époque que Viger conçoit l'idée d'un ouvrage sur les particularismes du français canadien. Il fait paraître dans l'édition du 7 janvier 1809 du Canadien un article, qu'il intitule déjà Néologie, dans lequel il traite du verbe bourgogner. Ce verbe, qui avait le sens de «battre», tire son origine du nom du général anglais Burgoyne, qui fut défait par les Américains en 1777 à Saratoga. Lorsqu'on connaît l'intérêt de Viger pour les faits d'armes, on ne peut s'étonner du choix de ce néologisme. L'article bourgogner figurera, remanié, dans la Néologie canadienne. 
La Néologie canadienne, qui constitue tant par la qualité des données qu'elle contient que par la date de sa rédaction un ouvrage lexicographique essentiel pour l'étude du français au Canada, reste cependant le seul travail de Viger sur l'analyse de la langue. Ce dictionnaire semble lui avoir tenu à coeur; en effet, les listes de mots et d'expressions que son ami le députépoète Ross Cuthbert lui a fournies pour enrichir sa nomenclature montrent à l'évidence qu'il a parlé de son projet et qu'il s'est intéressé à l'étude de la langue pendant quelques années.

Pourquoi n'a-t-il pas terminé cette étude alors qu'il avait déjà commencé une rédaction au propre qu'il comptait probablement publier? Des occupations ayant trait aux affaires publiques et d'autres études consacrées surtout à l'histoire, ont retenu son intérêt et l'ont sans doute détourné de son projet initial. On peut le regretter, car son activité lexicographique témoigne de la même ardeur et de la même minutie que celles qu'il a mises dans ses travaux sur l'histoire canadienne. Lors de la publication de la Néologie de Viger dans le Bulletin du parler fraņais au Canada, Camille Roy écrivait dans sa présentation : "Il fut lexicographe parce qu'il fut whistoriomane», parce qu'il eut toutes les sollicitudes d'un historien, et parce que le vocabulaire d'un peuple constitue l'une des pages les plus vivantes et les plus significatives de son histoire» (RoyViger 42).

C'est également en 1808 qu'il commence à colliger les documents qu'il utilisera pour écrire La Saberdache, son principal ouvrage. Ce dernier se divise en deux parties. La première, appelée Saberdache rouge, comprend 30 volumes qui portent sur l'histoire du Bas et du Haut-Canada; la seconde, la Saberdache bleue, totalisant 13 volumes, contient la correspondance de Viger, des notes et des papiers divers.

Après avoir décrit l'apparence physique de Viger, J.-G. Barthe nous explique la passion de Viger pour sa Saberdache:

Jacques [...] ne visait qu'à l'originalité à laquelle son visage sarcastique se prêtait beaucoup. Ses yeux, quelque peu fauves ou obliques, provoquaient le rire, et sa bouche enfantait l'épigramme qui en sortait parfois un peu brûlante, quand il s'agissait surtout de sa Saberdache, son enfant de prédilection, auquel il n'était pas permis d'attenter de près ni de loin. (Barthe, Souvenirs d'un demi-siècle, 402.)

L'inventaire de la Saberdache qui a été dressé par Fernand Ouellet et qui a été publié dans le Rapport de l'arcbiviste de la province de Québec facilite grandement l'accès à cette œuvre monumentale.

Bien qu'il n'ait pas voulu faire carrière comme député à la «Chambre d'assembléem, Viger, qui comptait des hommes politiques parmi ses proches - son père notamment fut élu député de Kent en 1796 (comté de Chambly depuis 1829) -, a toujours porté un grand intérêt à la politique. 
Comme l'écrit Jean-Claude Robert dans l'article qu'il lui consacre dans le Dictionnaire biographique du Canada: "Jacques Viger appartient au puissant réseau familial des Viger -Papineau - Lartigue - Cherrier. Cousin de Denis-Benjamin Viger, de Louis-Joseph Papineau, de Jean-Jacques Lartigue et de Côme-Séraphin Cherrier, il se tient durant toute sa vie en contact constant avec eux [...]” (RobViger 1011). Il a fait de la politique à sa manière en jouant auprès de ses amis engagés dans ce domaine le rôle de conseiller et d'informateur.

Viger fit néanmoins une brève incursion en politique municipale de 1833 à 1836, en se faisant élire à la mairie de Montréal dont il fut le premier maire.

Toujours très présent et très actif dans les différents domaines de la vie montréalaise, il fut le premier président de la Société Saint-Jean-Baptiste, fondée en 1834 par Ludger Duvernay. En outre, il fonda en 1858 avec quelques érudits, tels que les abbés Verreau, Ferland et Desaulniers, la Société historique de Montréal et en fut le premier président. À la mort de Viger survenue quelques mois plus tard, l'abbé Verreau lui succéda à la présidence.

Enfin, l'auteur de la Néologie, qui exerça tour à tour les fonctions de journaliste, de militaire, de fonctionnaire et d'épistolier fut avant tout un archiviste et un collectionneur infatigable. Il a légué à la postérité de nombreux écrits et documents sur l'histoire du Canada qui sont encore en grande partie inédits. Comme le fait remarquer Jean-Claude Robert : «Il n'est pas facile de saisir les multiples facettes de l'existence de Jacques Viger. Tant sa personnalité que l'ampleur de son activité et la diversité de ses intérêts en font un individu qui sort nettement de l'ordinaire» (ib. 1014). Il s'éteignit, après une vie bien remplie, dans sa ville natale, le 12 décembre 1858.

Tous les papiers et les écrits de Viger, dont la Néologie canadienne, furent acquis et classés par son confident et ami l'abbé Hospice-Anthelme Verreau. Á la mort de ce dernier survenue en 1901, ils furent déposés aux fonds d'archives du Séminaire de Québec.

\section{UN PIONNIER DE LA LEXICOGRAPHIE CANADIENNE}

Viger est, avec le père Pierre-Philippe Potier, le pionnier des études sur le français canadien. Leurs glossaires respectifs se situent à la charnière de deux époques. En effet, le travail de Potier intitulé Façons de parler qui a été rédigé de 1743 à 1758 , est de la fin du Régime français, alors que Viger a écrit la Néologie canadienne cinquante ans après la Conquête. 
Bien que la méthodologie et les objectifs des deux auteurs soient différents - le travail de Potier est essentiellement descriptif, tandis que Viger a des préoccupations d'ordre normatif -, ils ont cependant en commun l'amour des mots et le souci du détail. Rien n'est laissé de côté dans ces ouvrages; les auteurs consignent, en effet, des lexies qui touchent de nombreux domaines de la vie humaine, animale et végétale; enfin, toutes les classes de mots sont représentées. En outre, fidèles à la tradition dictionnairique des $\mathrm{XVII}^{\mathrm{e}}$ et XVIII ${ }^{\mathrm{e}}$ siècles, les auteurs n'hésitent pas à émailler leurs articles de commentaires, d'expressions, de proverbes et de comparaisons qui enrichissent considérablement leurs dictionnaires et en rendent la lecture agréable, réalisant ainsi le vou que formait $\mathrm{A}$. Boyer dans la préface de l'édition de 1773 de son dictionnaire : "Il seroit à souhaiter que l'on pût faire d'un Dictionnaire un ouvrage amusant.»

Viger connaissait-il les Fafons de parler du père Potier? Comme le fait remarquer Marcel Juneau, dans son étude qui traite des deux auteurs, certains indices le laissent à penser (JunLex 26). En effet, une trentaine de mots relevés par Viger figurent déjà dans Potier. Si tel était le cas, force est de constater que, s'il s'en est inspiré, il ne l'a pas copié. Les définitions et les exemples sont beaucoup plus développés dans le travail de Viger et témoignent d'une connaissance et d'une observation personnelles de la langue. Citons par exemple, en comparant avec Potier, le verbe bredasser auquel il ajoute un sens figuré et un mot de la même famille, soit bredas. En outre, il est difficile d'attribuer à Potier les mots dont la graphie est différente chez les deux auteurs, comme c'est le cas pour drigaille, ébrayer, otocas, que Potier orthographie drigail, ébreuiller, atoka.

Il convient de remarquer également que leurs préoccupations ne sont pas les mêmes. Alors que Potier qui partage la vie des Amérindiens consigne 42 termes et expressions d'origine amérindienne, Viger n'en retient que sept. Ce sont des mots déjà bien intégrés au français canadien et qui, pour la plupart, sont attestés depuis le XVII ${ }^{\mathrm{e}}$ siècle ou le début du $\mathrm{XVIII}^{\mathrm{e}}$ siècle, tels que acbigan, apichimon, caribou, maskinongé, micoine, mitasse et otocas.

Ces mots sont encore en usage, à l'exception d'apichimon qui semble avoir eu une existence éphémère; il est absent, entre autres, du glossaire de Clapin. Il faudra attendre ce dernier, qui partageait sûrement l'intérêt de Potier pour la culture amérindienne, pour que figure dans un glossaire un nombre aussi considérable d'amérindianismes.

Nous disposons maintenant du texte complet et fidèle du manuscrit du père Pierre-Philippe Potier qui a été édité par Peter W. Halford sous le titre Le franfais des Canadiens à la veille de la Conquête (v. bibliogr. HalPot). 


\section{LOBSERVATEUR DU LANGAGE}

La Néologie canadienne apporte aux études sur le français canadien le premier ouvrage à caractère normatif. Pour comprendre la démarche lexicographique de Viger, il faut se remettre dans l'esprit de l'époque et le milieu dans lequel il a vécu. Imprégné de culture française durant ses études au collège (les Sulpiciens qui y enseignaient étaient tous, à cette époque, natifs de France), Viger a fréquenté assidûment, par la suite, les gens de lettres; il eut comme amis, entre autres, les poètes français Joseph Quesnel et Joseph D. Mermet. Un article de Michel Bibaud sur la prononciation nous aide à saisir le type d'enseignement que Viger a reçu. Il nous apprend, d'une part, qu'au Collège de Montréal l'enseignement de la prononciation était dispensé par des professeurs français et que, d'autre part, la norme était celle de Paris. Il écrit :

Je regarde même ces fautes [de prononciation] comme presque impardonnables dans ceux qui ont eu occasion d'étudier au collége de Montréal, où l'on a l'avantage d'avoir des professeurs qui, s'ils ne sont pas nés à Paris, ont du moins résidé assez longtems dans cette capitale, pour prendre le ton de la belle prononciation Française. ( $L a$ Bibliothèque canadienne, $\mathrm{t} . \mathrm{I}, \mathrm{n}^{\circ} 5$, octobre 1825, p. 158.)

Par ailleurs, son travail de rédacteur au Canadien qui l'obligeait à réfléchir sur la langue, a certainement contribué à développer chez lui un esprit critique et à lui faire prendre conscience, en même temps, de l'écart qui existait entre le langage des gens instruits et celui de la majorité de ses compatriotes.

En outre, comme bon nombre de ses contemporains, Viger sentait sa langue menacée. Il faut savoir qu'à l'époque où il travaille au Canadien, l'un des sujets brûlants de l'actualité était la lutte que menaient les députés pour la reconnaissance officielle du français au Parlement. La menace était, en effet, réelle et venait de deux sources différentes. Le français canadien était menacé, d'une part, par son caractère archaïsant et l'était, d'autre part, par la présence de plus en plus envahissante de l'anglais. Ce sont, d'ailleurs, les mots appartenant à ces deux catégories qui seront la cible de ses critiques. Il était donc naturel que par son éducation, son milieu et son travail, Viger éprouvât le besoin de s"intéresser à la langue et surtout de prendre parti.

L'auteur ne se contente donc pas de décrire la langue de ses contemporains, il la confronte à une norme qui est celle du français de France consigné dans les dictionnaires. Les lexies qui ne figurent pas dans les dictionnaires, et en particulier dans celui de l'Académie, trouvent difficilement grâce à ses yeux, et les formules qui les condamnent sont sans appel. Ainsi il écrit sous attisée : «ce mot ne se dit pas"; sous beurrer: «Il n'est pas du tout françois»; sous ébrâiller: "Se débrâiller est le seul mot que la 
lang[u]e permette.» Comme nous le verrons, cette attitude intransigeante pour des mots et des sens qui pourtant viennent des parlers français s'explique, en partie, par un manque de connaissances historiques. Les glossaires dialectaux qui auraient permis à l'auteur de reconnaitre, parmi les mots retenus, ceux qui étaient d'origine dialectale et, qui lui auraient ainsi permis d'en retracer l'histoire, étaient inexistants au début du XIX ${ }^{\mathrm{e}}$ siècle. De plus, les dictionnaires des $\mathrm{XVII}^{e}$ et XVIII ${ }^{e}$ siècles en général et celui de l'Académie en particulier n'étaient pas très accueillants pour les termes et les expressions de la langue familière.

\section{L'OBJECTIF DE L'AUTEUR}

D'entrée de jeu, Viger énumère dans le titre qu'il donne à son ouvrage les catégories de mots et d'expressions qu'il compte retenir: Néologie Canadienne, ou Dictionnaire des mots créés en Canada Ė maintenant en vogue; - des mots dont la prononciation E' l'ortographe sont différentes de la prononciation $\mathcal{E}^{2}$ ortographe françoises, quoique employés dans une acception semblable ou contraire; et des mots étrangers qui se sont glissés dans notre langue.

La première catégorie comprend les innovations formelles ou sémantiques du français canadien. Viger accepte cette catégorie de mots qui répond à la nécessité d'innover tant du point de vue lexical que sémantique pour décrire les réalités canadiennes. C'est ainsi que les mots canotée, poudrer, poudrerie, tire, tuque, entre autres, sont analysés avec objectivité. Cette catégorie semble faire l'unanimité chez les glossairistes canadiens; ainsi Maguire en 1841 écrit :

Tous les lexicographes conviennent de la nécessité d'incorporer à la langue les termes de relation qui expriment les choses et les objets qui n'existent que dans les pays lointains, nouvellement découverts, ou avec lesquels l'on a eu peu de communications. D'où il résulte pour le Canada le droit de créer des termes pour les objets et les choses qui lui appartiennent exclusivement. (Maguire sous Néologie 70.)

La deuxième catégorie englobe les mots français qui ont en français canadien des variantes phonétiques, orthographiques ou sémantiques; enfin, la troisième comprend les emprunts à l'amérindien et à l'anglais.

Une lecture attentive du titre fait découvrir une lacune dans le classement des lexies. En effet, cette énumération laisse dans l'ombre les archaïsmes ainsi que les dialectalismes qui forment pourtant dans le manuscrit un groupe important de mots d'origine gallo-romane. Si l'absence de classement n'altère en rien la qualité et la pertinence des informations que nous livre Viger dans les articles, elle se fait néanmoins sentir dans les commentaires dont l'auteur est prodigue et conduit quelquefois 
à des conclusions incomplètes sinon erronées. Ainsi, il écrit sous gueusaille, gueusasse (Ms. 2) : "On se sert également de ces deux mots, quoique gueusaille soit le seul françois»; de même, sous ramancher au sens de «conter, ou raconter avec diffusion", le verbe est donné comme «canadien», alors que dans cette acception ramancher est un dialectalisme attesté dans les parlers du Nord-Ouest et de l'Ouest.

Pour les raisons que nous venons d'énumérer, Viger a dû travailler en synchronie. Dès lors, il était inévitable que le classement des lexies, coupé du lien historique, comporte des erreurs d'interprétations. C'est ainsi que des mots comme attisée, beurrer, gueusasse, ramancher et beaucoup d'autres ne sont pas classés ou figurent dans une catégorie qui n'est pas la leur.

C'est également ce qui explique que les mots espérer, marier et plaisant, figurent parmi les anglicismes, alors qu'il s'agit d'archaïsmes qui vivent encore dans certains parlers français. Viger est le premier d'une très longue liste de commentateurs de la langue à fournir la preuve que, pour être scientifique, le classement des mots doit s'appuyer sur une solide analyse historique des données linguistiques. Cette analyse est d'autant plus nécessaire que la distinction entre anglicismes et archaïsmes ou dialectalismes n'est pas toujours facile à faire.

Il ne fait aucun doute que l'anglais a joué un rôle dans le maintien de certains archaïsmes et dialectalismes en français québécois et canadien, mais cela ne fait pas pour autant de ces derniers des anglicismes. Une analyse historique approfondie est toujours nécessaire dans ce domaine, et spécialement lorsque le mot français qui fait l'objet de l'étude a un paronyme en anglais comme c'est le cas pour les trois exemples cités précédemment. Bref, l'avancement des recherches historiques, soutenu par un nombre important d'ouvrages remarquables sur le sujet rend peu pertinentes, de nos jours, les remarques de Viger en ce qui a trait au classement des lexies. Lintérêt de son travail réside avant tout dans la somme d'informations qu'il fournit et dans ses qualités d'observateur de la langue.

Les premiers glossaires sur les dialectes français ont vu le jour dans la deuxième moitié du XIX ${ }^{\mathrm{e}}$ siècle. Ceux-ci vont apporter, en France comme au Canada français, une dimension nouvelle aux études historiques du français. Ils vont pour ainsi dire donner leurs lettres de noblesse à des lexies que certains glossairistes, dont Viger, jugeaient inacceptables.

Oscar Dunn, dans son Glossaire franco-canadien publié en 1880, sera le premier à faire ressortir l'origine dialectale des mots et des expressions qu'il a relevés. Il écrit à ce sujet :

Nous employons un bon nombre de mots qui, rejetés par l'Académie, nous sont venus toutefois de France; ils appartiennent à quelque patois. On trouvera dans ce glossaire le premier relevé qui en ait été 
fait. [...].Toutes ces expressions prouvent notre origine; elles sont autant de certificats de nationalité. Aussi je me flatte qu'au point de vue ethnologique, ce travail aura un certain intérêt. (Dunn XIX-XX.)

La comparaison de quelques articles qui paraissent respectivement dans Viger et dans Dunn illustre de façon éloquente le changement d'attitude qu'a provoqué, à la fin du XIX ${ }^{\mathrm{e}}$ siècle, la prise de conscience de l'origine gallo-romane de nombreux mots et expressions qui constituent le français québécois et canadien. Alors que dans l'article attisée Viger écrit : «ce mot ne se dit pas», Dunn, qui a trouvé à ce mot une origine dialectale, plaide pour son maintien dans l'usage : "Une bonne attisée" est un mot bien formé qui existe dans quelques provinces en France; conservons-le»; de même, sous étriver (Ms. 2), Viger termine son article par cette phrase : «Etrivant \& Etriver ne sont françois ni l'un ni l'autre»; sous le même terme Dunn écrit: «Vieux mot qui signifie Lutter. Nous disons comme en Normandie, Faire étriver quelqu'un dans le sens de Plaisanter, gouailler, gausser, railler, taquiner. En Picardie, Contrarier.»

Enfin, comme la plupart des intellectuels de son temps, Viger s'attaque à l'anglicisme et plus particulièrement à l'anglicisme sémantique qui compte pour environ la moitié de la trentaine d'anglicismes que l'auteur a retenus. Pour décrire l'anglicisme sémantique, il a cette phrase, sous marier (Ms. 2), qui sera reprise maintes fois sous différentes formulations : "C'est employer, on ne peut pas mieux, des mots françois à parler anglois.»

Viger voit l'anglicisme comme une manie qui l'agace et il l'attaque par le sarcasme. Il ressort des commentaires qui émaillent ses articles, que l'anglicisme est une mode qui relève d'un certain snobisme : "mot anglois en vogue», «c'est un adjectif à la modem, «il commence à être beaucoup en vogue ici", "c'est un mot favori", «il est du haut ton", «il est de mode chez grand nombre de personnes de dire [...]».

Il déplore que cette mode atteigne les personnes qui devraient soit par leur instruction, soit par leur milieu, l'éviter : «Beaucoup de nos puristes du bon ton disent avec satisfaction [...]", "et l'on dit dans la bonne compagnie même $[. .$.$] », «plusieurs personnes et surtout du bon ton vous disent [. .$.$] »,$ «il [le verbe originer] n'est pas françois, quoique la magistrature même s'en serve ici, et qu'on ait tout lieu de croire qu'il ne vient point d'une source ignoble; car ce mot n'est d'usage que parmi nos gens comme il fautm.

Il y voit un goût pour la nouveauté, pour ce qui vient d'ailleurs; après avoir dénoncé le sens anglais du terme office, il ajoute ironiquement : «Les mots étude, bureau, cabinet, sont trop vieux \& trop communs!»; et dans l'article payer: «Dans ce sens, Faire est trop vieux, sans doute! de plus il est françois! Et Rendre n'est pas familier à ces gens». 
En 1802, le poète Joseph Quesnel abonde dans le même sens. Dans sa pièce intitulée L'Anglomanie ou le Diner à l'angloise, il dénonce, dans un style badin, l'engouement que manifeste une certaine classe de la société pour la langue et la culture anglaises.

Quelques années plus tard, Michel Bibaud parlera à son tour de «manie d'anglifier le français»:

Rien ne dépare tant un idiome que les mots et les tours barbares qu'on y introduit mal à propos; et les personnes qui ont à cœur la pureté de leur langue, devraient reprouver de tout leur pouvoir, et tourner en ridicule, cette manie d'anglifier le français, qui parait devenir plus générale de jour en jour. (L'Aurore, 18 avril 1818, p. 62.)

C'est dans le même style qu'en 1841 Thomas Maguire critiquera l'attitude des Canadiens français face à l'anglais :

Quant à l'emploi de mots purement anglais, là où il y a des termes en français qui leur correspondent, c'est une manie insupportable, c'est le comble du ridicule; et cependant combien de personnes, même d'éducation, qui tombent dans ce défaut! (Maguire 71.)

Bien qu'empreintes de subjectivité, caractéristique de la pensée et du style de Viger, ces formules ont néanmoins le mérite de nous faire connaitre les habitudes langagières des citadins canadiens-français de l'époque et leur perception de l'anglais. Comme cette langue était véhiculée, au début du XIX ${ }^{\mathrm{e}}$ siècle, par les gens instruits et par les journaux, son influence se faisait d'autant plus sentir, d'où les dénonciations répétées et virulentes des intellectuels. Il faut cependant spécifier que ces auteurs, y compris Viger, décrivent principalement le parler urbain, c'est-à-dire celui de Québec et surtout celui de Montréal.

Dans la seconde moitié du XIX $\mathrm{X}^{\mathrm{e}}$ siècle, l'industrialisation fera proliférer les anglicismes, sous l'influence de l'anglo-américain. Ces anglicismes se répandront dans toutes les sphères de l'activité économique et dans toutes les classes de la société.

\section{LES MANUSCRITS}

Le travail lexicographique que nous a laissé Jacques Viger sur un certain nombre de particularismes de la langue française au Canada, au début $\mathrm{du} \mathrm{XIX}^{\mathrm{e}}$ siècle, comprend deux grands cahiers et vingt petits cahiers manuscrits; à ces cahiers s'ajoutent quinze feuilles volantes où figurent des listes de mots et d'expressions, dont une grande partie n'est pas de Viger. Ces documents sont classés au fonds Verreau, $\mathrm{n}^{\mathrm{O}} 67$, liasses I B et 2 , au Musée de la civilisation, fonds d'archives du Séminaire de Québec. 
La Néologie canadienne est contenue dans deux manuscrits : le manuscrit de travail que nous appelons manuscrit 1 et qui comprend deux grands cahiers, et un second que l'auteur voulait peut-être définitif, que nous nommons manuscrit 2 et qui comprend vingt petits cahiers.

\section{LE MANUSCRIT 1}

Les deux cahiers du manuscrit 1, que l'auteur appelle Livre A et Livre $B$, sont identiques dans leur présentation et se complètent. Le format est le même; les articles sont traités de la même façon et ne se répètent pas. Lorsqu'une lexie est consignée dans les deux cahiers (c'est le cas de bougon, côte, fine-boutique et moindrement), cette répétition a toujours pour but d'ajouter des précisions, des sens nouveaux ou des exemples. Leur complémentarité est évidente; l'auteur n'a recours au Livre $B$ que lorsque l'espace fait défaut dans le Livre $A$.

Les feuilles des cahiers mesurent $36 \mathrm{~cm}$ de large sur $30 \mathrm{~cm}$ de haut; elles sont pliées dans le sens de la largeur, ne sont pas paginées et comportent des pages vierges. Les feuillets sont écrits au recto et au verso, sur deux colonnes. Le texte, qui contient de nombreuses ratures, est écrit à l'encre et quelquefois au crayon; l'écriture est tantôt très fine et régulière, tantôt plus relâchée, de lecture généralement assez facile. Le Livre $A$ porte la date du 12 novembre 1810 ainsi que le titre. Viger n'a pas signé son texte. La signature qui figure au-dessous du titre n'est pas de lui. Lécriture est d'un module différent de celle de Viger; en outre, elle est à l'encre bleue, contrairement au reste du texte qui est à l'encre brune.

\section{LE MANUSCRIT 2}

Par sa présentation, le manuscrit 2 a l'apparence d'un texte définitif. La disposition des articles et l'écriture sont soignées ainsi que la rédaction de l'ouvrage dans son ensemble qui comporte peu de ratures. Les articles sont généralement beaucoup plus développés que ceux des Livres $A$ et $B$; les sens sont mieux classés, le style est plus recherché. Tous les articles, à l'exception de celui qui porte sur le mot finition, qui est ajouté dans ce manuscrit, figurent dans le manuscrit 1 . Viger a indiqué par le signe $\mathbf{x}$ devant le terme vedette, les articles du manuscrit 1 qui figurent dans le manuscrit 2. Ils sont beaucoup moins nombreux, l'auteur n'ayant retenu dans ce manuscrit qu'environ le tiers des articles consignés dans le manuscrit 1 , soit 144 entrées.

Le manuscrit 2 comprend vingt petits cahiers. Chacun des cahiers est composé de deux feuilles de $34 \mathrm{~cm}$ de large sur $20,5 \mathrm{~cm}$ de haut pliées dans le sens de la largeur. Les articles sont insérés dans un encadré de $15,5 \mathrm{~cm}$ sur $11,5 \mathrm{~cm}$ tracé au crayon. Chaque cahier est consacré à une lettre de l'al- 
phabet. La pagination qui figure sur les feuillets n'est pas de Viger. Ce manuscrit ne porte ni titre ni date.

Si l'auteur se montre accueillant dans le manuscrit 1 , il est, par contre, beaucoup plus sélectif dans le manuscrit 2 . Sont écartés systématiquement de ce manuscrit les termes qui ne constituent qu'un écart de prononciation, tels que âbre, cartron, chardron, deusse, troisse, etc. Lauteur précise sa pensée à ce sujet dans l'article amancher (Ms. 2) : «Si ce verbe n'étoit jamais employé que dans son sens propre, celui d'emmancher, - et que l'on ne commit d'autre faute que de le prononcer mal, je ne m'arrêterois peut-être pas à en faire ici mention.» Néanmoins, le souci de rendre compte de la prononciation ne le quitte pas. Cela se traduit dans la graphie de certains mots, dans lesquels l'accent circonflexe qui indique le [o] postérieur sombre a été conservé, comme dans abât, brâsser, ébrâiller, gâa, gouliâ.

Les termes qui ne constituent qu'un écart de genre ou de nombre n'ont pas été retenus dans ce manuscrit; n'y figurent pas les mots araignée, argent, incendie; confessionnals, fanals, etc.

Exception faite des mots fenouil, fenouillette, ginseng (ce dernier a été retenu parce qu'il figure dans l'expression tomber comme le ginseng) et orignal, les termes qui se rapportent à l'histoire naturelle n'entrent pas dans le manuscrit 2. D'ailleurs, la place qu'ils occupent dans le manuscrit 1 -ils sont toujours en bas de page, ne sont pas définis ou le sont sommairement - incite à penser que l'auteur ne comptait pas, au début de son travail, les intégrer à son dictionnaire.

Les anglicismes lexicaux et sémantiques sont au nombre d'une trentaine dans le manuscrit 1; ne seront retenus dans le manuscrit 2 que les anglicismes sémantiques. Déjà, dans le manuscrit 1, l'auteur fait une distinction dans la présentation des deux types d'anglicismes. Alors que l'anglicisme lexical est généralement décrit comme un "mot anglois», l'anglicisme sémantique a droit à des commentaires souvent assez longs.

Le texte des articles est plus soigné, plus explicite. L'auteur a refait un grand nombre de définitions, notamment dans les articles côte, butin. Il a ajouté des sens et les a mieux classés en les numérotant, comme dans les articles canotée, débarquer, démancher, fine-boutique, peter pour n'en citer que quelques-uns. Les exemples sont également plus nombreux, notamment sous boucane, boucaner, exemple, mistimus, quitte, vulgaire. Le style est plus soigné; les articles bombe et tinton sont caractéristiques à cet égard.

\section{LES SOURCES}

Une bonne partie de la nomenclature de Viger repose sur l'observation personnelle de la langue orale; les nombreux commentaires sur la 
fréquence d'emploi et sur les niveaux de langue l'attestent. Il a puisé également dans quelques sources écrites parmi lesquelles nous avons pu identifier les Fafons de parler du père Potier; les listes de mots et d'expressions fournies par Ross Cuthbert et que nous avons reproduites dans les appendices; Travels and Adventures in Canada d'Alexander Henry dont il recopie des extraits (v. document III des appendices); en outre, comme Viger était aussi journaliste, on peut citer parmi les sources certains journaux.

\section{ORGANISATION DES ARTICLES}

Le travail de Viger se présente comme un dictionnaire. L'auteur s'est inspiré, à maints égards, des dictionnaires des XVII et XVIII ${ }^{e}$ siècles. Cela ressort notamment dans l'organisation et la présentation des articles et dans les formules utilisées dans la métalangue; cette influence se manifeste également en ce qui a trait à la calligraphie, comme par exemple l'emploi du $s$ long $(S)$ lorsque cette consonne est redoublée à l'intérieur d'un mot, de la perluète $\left(\xi^{*}\right)$ qui remplace généralement la conjonction et dans les manuscrits et de et cetera, abrégé en Eंc.

Nous ne connaissons des dictionnaires quil a consultés que les trois qui sont nommés dans le corps de son travail; il cite le dictionnaire de l'Académie (sans donner l'édition) et deux dictionnaires bilingues soit celui de Pierre Danet, Grand Dictionnaire franfois et latin (qui connut six éditions de 1673 à 1742) et celui d'Abel Boyer, Dictionnaire royal franfoisanglois et anglois-franfois (qui eut de nombreuses éditions avant le XIX ${ }^{\mathrm{e}}$ siècle).

Bien que Viger n'ait pas donné l'édition du dictionnaire de l'Académie qu'il a consultée, certains indices nous portent à croire qu'il s'est servi de la cinquième édition, publiée en 1798 (à noter que l'abrégé de cette édition qui date de 1821 figure dans la liste des livres de sa bibliothèque dressée en 1835). En effet, dans l'article ouète, il écrit que le dictionnaire de l'Académie indique deux prononciations pour ce mot, soit ouate et ouète ; or, la mention de ces deux prononciations figure pour la première fois dans la cinquième édition.

Pour notre part, nous avons pu consulter, outre les éditions de l'Académie, l'édition de 1735 du dictionnaire de Danet et l'édition de 1773 de celui de Boyer.

Viger connaissait-il le dictionnaire de Louis-Sébastien Mercier publié en 1801? On peut le penser, car le début du titre que Viger a donné à son travail, soit Néologie canadienne, ou Dictionnaire des mots créés en Canada, rappelle étrangement celui de Mercier, Néologie, ou Vocabulaire de mots nouveaux, à renouveler, ou pris dans des acceptions nouvelles. 
Les mots vedettes sont regroupés par lettres, mais ne sont pas rangés dans un ordre alphabétique rigoureux. Cela ne devait pas être une préoccupation pour l'auteur puisque, dans le manuscrit 2 , alors qu'il avait toute latitude de classer soigneusement par ordre alphabétique les mots qu'il retenait du manuscrit 1 , il ne l'a pas fait.

La graphie des mots vedettes suit généralement l'orthographe actuelle. En outre, il convient de signaler, à ce sujet, la normalisation que pratique l'auteur dans certains mots au détriment de la forme usuelle. $⿱ 亠 乂$ titre d'exemple, citons les noms et les adjectifs qui ont une désinence en $e u x$ et que l'auteur donne avec celle en -eur, peut-être influencé par l'orthographe des dictionnaires, notamment pour quêteur, qui est attesté depuis le $\mathrm{XV}^{\mathrm{e}}$ siècle. Il s'agit de bourasseur, fignoleur, quêteur, que les principaux glossaires consignent avec la désinence en -eux. Ne serait-ce pas que déjà, au début du XIX ${ }^{\mathrm{e}}$ siècle, cette désinence était sentie comme vieillie?

La graphie de certains mots vedettes tient compte de la prononciation; c'est le cas notamment de mots tels que âbre, gâa, gouette, etc. Ce procédé n'est pas propre à Viger; il est, en effet, très ancien et a fait l'objet d'âpres discussions chez les lexicographes des XVII ${ }^{\mathrm{e}}$ et XVIII ${ }^{\mathrm{e}}$ siècles. À ce sujet, Bernard Quemada remarque : «Ce sont les dictionnaires anglaisfrançais qui furent les premiers à pratiquer systématiquement ces notations. En 1792, par ex., la $16^{\mathrm{e}}$ éd. du Boyer fondait l'essentiel de sa publicité sur la présence d'indications phonétiques [...]» (QuemDict 119). D'autres lexicographes ont tenté l'expérience; citons notamment Féraud et Gattel, qui donnent boâ pour rendre la prononciation de bois (ib.).

Les indications étymologiques sont inexistantes et nous n'avons pas à le regretter, vu la difficulté que présente, pour l'amateur, cette partie de la linguistique. En revanche, l'origine des mots est généralement donnée. Les mots d'origine amérindienne sont accompagnés de la mention «mot sauvage», «vient du sauvage», «mot sauvage reçu dans la langue françoise». Les anglicismes sont identifiés comme "mot anglois", "ce mot est Anglois" ou simplement «anglois». Quant aux origines françaises des lexies et des sens, l'auteur n'est pas très explicite; il se contente d'indiquer que tel mot ou tel sens "n'est pas français" ou "ne se dit pas"; les termes archaïsme, dialectalisme, innovation ou création lexicale ne font pas partie de sa terminologie. Au lieu d'employer le mot archaïsme, l'auteur emprunte la terminologie de l'époque et écrit notamment sous flambe : «Vieux mot».

Les catégories grammaticales sont systématiquement indiquées et l'auteur utilise la terminologie employée dans les dictionnaires du XVIII siècle; ainsi, il écrit substantif, abrégé en sub. ou s., plutôt que nom, et verbe actif pour transitif, verbe neutre pour intransitif. 
Les définitions sont généralement assez précises ; le développement de certaines d'entre elles apporte un éclairage précieux, particulièrement dans le cas des termes qui ont connu ou connaissent encore des nuances sémantiques. Il en est ainsi du verbe bavasser qui a eu le sens de "parler à tort et à travers» et qui est connu de nos jours au sens de «bavarder» et «dire des médisances»; Viger précise : «Il ne se dit guères que d'une personne ivre ; et l'on dit: laissons-là, elle ne fait plus que bavasser, pour exprimer en même tems qu'elle parle beaucoup, confusément, sans savoir ce qu'elle dit, en prononçant imparfaitement $\&$ articulant avec peine». De même, sous cabrouet : «Voiture à deux roues, longue et étroite dont nos charetiers se servent»; sous pipe : «espéce de mesure de distance chez nos habitans : c'est à peu-près trois lieues»; sous sucet : «On appelle ainsi le tuyau du blé d'Inde, ou sa tige, dégarnie de ses épis, et que l'on fait manger au bétail à corne[s].»

Pour d'autres termes, l'équivalent français tient lieu de définition; ainsi sous trempe : «au lieu de trempé, ée»; il renvoie également à un synonyme français sous mècher: «Il équivaut à Rosser».

Les synonymes remplacent généralement la définition dans les mots qui décrivent la faune et la flore; par exemple, sous caribou: "pour, le renne»; sous oiseau blanc: «pour Ortolan»; sous passe-rose : «pour rosed'outremer, ou rose-tremièren. Bien qu'il ne soit pas satisfaisant, ce procédé permet néanmoins d'identifier les animaux et les plantes étudiés, puisque l'auteur ne fait jamais appel à la nomenclature scientifique dans son travail de définition de cette catégorie de mots.

Par ailleurs, Viger met la définition entre parenthèses lorsque le terme vedette est un mot du français général qui connaît une extension sémantique en français canadien; cette définition est celle du mot en français général, comme par exemple dans les articles appointement, brâsser, cabot, cantine, démancher, escabeau, espérer, fesser, flandrin, gaspiller, etc. La définition entre parenthèses est aussi celle du français général lorsque le mot vedette ne représente qu'une variante phonétique ou formelle; il est généralement suivi de l'équivalent français; on peut citer ici les entrées bèr, brunante, dégelée, écopeau, fard, lévier, racérer, retontir, etc. En résumé, sauf quelques exceptions, comme écrapoutir, graffigner, manchon[n]ier, qui sont des dialectalismes, ce procédé n'est utilisé que pour définir des mots du français général. Cette présentation est empruntée notamment à Boyer qui met systématiquement la définition des mots vedettes entre parenthèses.

Des exemples nombreux et bien choisis viennent éclairer la définition. L'auteur avait compris la pertinence de mettre en situation contextuelle les mots et expressions qu'il retenait. Ainsi, sous moucher, les exemples viennent préciser que l'action de corriger est dirigée vers un enfant : "Son père l'a bien mouché ; fanfan, je te moucherai, si tu ne te tais.» 
Par ailleurs, les exemples nous renseignent sur les mœurs de l'époque; ainsi sous fesser au sens de "fouetter» : «Un tel est condamné par la Cour à être fessé tel jour, sur la place du marché.»

Les exemples qui s'appliquent aux termes payer et style notamment mettent le lecteur dans l'ambiance sociale du début du XIX ${ }^{\mathrm{e}}$ siècle : «j'irai demain, Monsieur vous payer une visite»; «je viens de chez Madame lui payer mes respects mes complimens»; «la table de Monsieur étoit servie dans le plus haut stile»; «sa maison est meublée dans le grand stile»; "madame est habillée dans le grand stile"; sous rôle, "petits pains que l'on vent [sic] dans les rues le matin \& le soir, pour le thé»; «le crieur de miches est à la porte».

La syntagmatique tient une place également importante; parmi les nombreux exemples, on peut citer abât de neige, de pluie ; beurrée de graisse, de sirop, de crème; bougon de chandelle, de pipe; sieau ferré, des sieaux de bois; de bonne tire, de la tire pleine d'yeux.

La polysémie des mots n'a pas échappé à l'auteur. Il a vu l'importance de bien distinguer les différents sens d'un terme, en particulier dans le manuscrit 2 , où il les a numérotés, comme dans les articles comfortable ( 5 sens), débarquer (4 sens), démancher (6 sens), peter (6 sens).

En outre, lorsqu'un mot du français général connait une extension sémantique en français québécois, l'auteur a souvent recours au sens français afin de faire ressortir l'écart sémantique du mot relevé comme, par exemple, dans l'article mouiller (Ms. 2) : «Mouiller. v. n. On l'emploie ici presque exclusivement à celui de pleuvoir [...]. Dans ce cens, mouiller ne peut absolument pas se dire. Mouiller est un verbe actif, qui ne s'emploie jamais en françois, que pour marquer l'action de tremper, humecter, rendre humide."

Les rapprochements onomasiologiques ont également leur place dans les articles; l'auteur cherche constamment à établir un lien entre les mots, soit à l'intérieur des articles, soit par le système des renvois. Citons par exemple bombe, canard et bouilloire; abât, bordée et giboulée; bicleux, loucheur et vire-l'ail; bougon, nijon et bout d'bomme; bombarde, trompe et guimbarde; berline, bordel, cariole et traineau; cage, cajeux, crible et dram; moucher et mècher; cordeaux, courroies, guides et rênes.

En outre, l'auteur indique pour certains de ces groupes de mots les nuances d'emploi qui les caractérisent; ainsi, on peut lire sous bordée (Ms. 2) : «On dit aussi absolument : il a tombé hier une grande bordée. Car la bordée s'applique à la nége seule; tandis que l'abât se dit de la nége et de la pluie"; sous moucher : "Il a la même signification que mècher, avec cette différence pourtant qu'on l'emploie plutôt contre un enfant que contre un homme fait, ou un animal»; sous berline, bordel : «Le Bordel est la plus grossière de ces voitures». 
Les niveaux de langue sont quelquefois indiqués; les formules utilisées sont celles des lexicographes des XVII et XVIII siècles : «ces mots sont bas», «mot bas \& populaire», etc. Cependant, l'auteur n'en fait pas un emploi généralisé, préférant se servir de phrases personnelles : «mot employé par les habitans», «on lui donne parmi le peuple», «plusieurs personnes \& surtout du bon ton vous disent", "ce mot est de la bonne compagnie», etc.

La fréquence d'emploi retient également son attention et ajoute encore à la qualité du travail : sous abât (Ms. 2) : "Il est fort commun d'entendre dire»; sous fanals: "pluriel de fanal usité communément au lieu de fanaux "; sous fard, l'auteur commente ainsi l'emploi du mot farce : «Bien peu de personnes employent ici le vrai mot»; il fait une remarque similaire pour le mot bouilloire sous bombe (Ms. 2): «Le dernier de ces mots n'est pas du tout en usage»; sous improuver (Ms. 2) : «On dit fort généralement dans nos villes»; sous joli-caur : «Ce mot [...] s'emploie tous les jours dans les phrases suivantes"; sous mondaine (orge mondaine) : «Il n'est employé [que] par quelques personnes»; sous payer (Ms. 2) : «Il est de mode chez grand nombre de personnes de dire"; sous plairie : "quelquefois usité pour Prairie»; sous prouvable (Ms. 2) : «L'emploi de ce mot est très commun ici».

Enfin, Viger s'est intéressé également à la géographie linguistique. Son passage à Québec lui a certainement donné l'idée de localiser certains synonymes; ainsi sous amarrer: «A Québec surtout ce mot est employé à tout moment»; sous amont la côte: "Manière de parler plus particulière au district de Québec"; sous bombe: "mot usité plus particulièrement dans le District de Québec [...]. Dans le District de Montréal, on employe dans la même signification le mot Canard"; sous travail: "Le mot travail est employé à Montréal \& celui de menoirs au pluriel à Québec». Les enquêtes effectuées pour l'Atlas linguistique de l'Est du Canada (PPQ), dans les années 1970, montrent encore pour les mots canard et bombe une distribution géographique qui se rapproche de celle donnée par Viger (PPQ170).

\section{LÉDITION DE LA SOCIÉTÉ DU PARLER FRANÇAIS AU CANADA}

La Néologie canadienne a été publiée au début du siècle par la Société du parler français, soit cent ans après sa rédaction. Le texte a paru, par tranches, dans le Bulletin du parler franf̧ais au Canada, vol. VIII, 19091910 , p. $101-103$; 141-144; 183-186; 234-236; 259-263; 295-298; 339342. Malheureusement, l'effort qui, en soi, était louable manque de rigueur scientifique et rend cette édition peu fiable et, par conséquent, pratiquement inutilisable en raison de sa conception même qui repose sur une erreur de méthodologie dans la présentation et la rédaction des articles. 
L'éditeur explique ainsi le choix qu'il a fait :

Quoi qu'il en soit, on a cru devoir ici, pour plus de commodité, et aussi pour tirer du travail de Viger le meilleur parti possible, fondre ensemble les trois manuscrits en observant rigoureusement l'ordre alphabétique. Lorsque sur les cahiers de Viger on a trouvé deux définitions d'un même mot, ce qui est rare, c'est la plus claire, la plus précise, celle qui a paru la meilleure que l'on a choisie. (RoyViger 54.)

L'erreur la plus grave et qui dénature le texte de Viger est, sans contredit, celle qui a consisté à retoucher un grand nombre d'articles. En adoptant le principe de fusion des manuscrits, l'éditeur a dû récrire certains articles. C'est ainsi qu'un grand nombre d'entre eux furent soit refaits, soit amputés de mots, voire de phrases complètes.

En pratique, le texte qui fut généralement retenu est celui du manuscrit 1 qui n'est pas toujours le meilleur ni le plus complet. Le texte de l'article $a b a t$, tiré du manuscrit 1 , ne donne comme emploi que le syntagme abât de neige, tandis que dans le manuscrit 2 la syntagmatique s'enrichit d'abât de pluie; l'article confortable (Viger a écrit comfortable suivant en cela la graphie anglaise) est amputé également des précisions données par Viger sur l'emploi du mot en français et en anglais; dans l'article embarquer, tiré également du manuscrit 1 , le lecteur est privé d'un commentaire essentiel de Viger qui figure dans le manuscrit 2: "Parler ainsi n'est pas parler françois». On pourrait multiplier les exemples.

Certains articles n'ont pas été retenus. Ainsi ont été écartés, sans explication, les mots quêter et quêteur; flasquer au sens de «repasser» que Viger donne dans fer à flasquer; frète, frède adjectifs, etc.

Pourquoi avoir adopté, dans les entrées comme dans les articles, l'orthographe actuelle alors que celle de Viger ne présente aucune difficulté de lecture? Les variantes anciennes du texte de Viger portent sur un certain nombre de mots tels que françois, anglois, habitans, enfans, ortographe, byver, etc., qu'il était indispensable de conserver en raison de l'intérêt qu'elles présentent pour l'histoire de l'orthographe.

En outre, le texte contient un nombre incalculable d'erreurs de lecture qui surprennent d'autant plus que l'éditeur a travaillé sur des manuscrits rédigés avec soin et bien conservés. Marcel Juneau en a relevé un certain nombre dans son étude critique de cette édition et nous y renvoyons le lecteur (JunLex 24).

Signalons enfin que l'éditeur n'a pas tenu compte des listes de mots et d'expressions qui accompagnent les manuscrits de Viger et qui auraient certainement figuré dans son ouvrage s'il l'avait complété. Ces mots et ces expressions, fruits d'une observation méticuleuse du parler des Canadiens 
français du début du XIX ${ }^{\mathrm{e}}$ siècle, représentent souvent des premières attestations en français québécois et canadien.

\section{LA PRÉSENTE ÉDITION}

La difficulté que présente l'édition de la Néologie canadienne tient au fait que ce travail comporte deux manuscrits. Fallait-il publier le manuscrit 2, le mieux rédigé mais le moins complet, ou valait-il mieux publier le premier manuscrit, le manuscrit de travail, beaucoup plus complet, et renvoyer le manuscrit 2 dans l'apparat critique? Il s'agit ici de deux manuscrits dont l'un, le second, puise dans le premier, il est vrai, mais en le modifiant tant dans le contenu que dans la forme. La question ne se poserait pas si les deux manuscrits étaient de même niveau; il aurait été facile et tout indiqué de les fusionner. Comme Viger, dans le manuscrit 2, a rédigé un texte soigné en y ajoutant des précisions et des commentaires, il était, dès lors, difficile de reléguer ce manuscrit au second plan.

Par fidélité au texte et afin de ne pas retomber dans les erreurs de la Société du parler français, nous avons choisi de publier intégralement les deux manuscrits. Nous présentons d'abord le manuscrit 1 , le moins parfait mais le plus complet et le plus intéressant par la richesse de sa nomenclature qui comporte de nombreux termes se rapportant notamment au monde rural ainsi qu'aux sciences naturelles et par les nombreuses informations phonétiques, morphologiques et syntaxiques qu'il fournit; suit le manuscrit 2 présenté intégralement.

Ce choix permet de prendre connaissance, en peu de temps, de la composition de chacun des manuscrits et de suivre le travail de l'auteur au cours de la rédaction de son ouvrage. La comparaison des deux manuscrits permet de mieux saisir les préoccupations linguistiques du début du XIX ${ }^{\mathrm{e}}$ siècle. Par exemple, le fait que Viger a retenu, dans le manuscrit 2, tous les anglicismes sémantiques du manuscrit 1 alors qu'il a éliminé un très grand nombre de mots de ce manuscrit, est déjà une piste intéressante à suivre.

La Néologie canadienne de Jacques Viger que nous éditons aujourd'hui pour la première fois dans son intégralité s'inscrit dans la tradition des travaux de recherches historiques en français québécois qui ont vu le jour à l'Université Laval dans les années 1970, sous l'impulsion de Marcel Juneau.

\section{ÉTABLISSEMENT DUTEXTE}

Jacques Viger est un lettré et un érudit du début du XIX ${ }^{\mathrm{e}}$ siècle. Il a l'habitude des dictionnaires et cela se voit dans son travail. Il connaît bien la grammaire, ce qui signifie que les fautes d'accord du nom, de l'adjectif 
ou du participe notamment ne sont pas très nombreuses et sont dues plutôt à l'inattention qu'à l'ignorance. Les écarts orthographiques que nous signalons sont pour la plupart des coquilles ou des oublis; cependant, un bon nombre d'entre eux relèvent de graphies anciennes qui s'expliquent d'ailleurs historiquement.

Nous présentons le texte de Viger tel qu'il l'a rédigé. Nous n'avons rien retranché comme nous n'avons rien ajouté dans la nomenclature et dans les articles. Même les articles raturés par l'auteur ont été conservés; nous le signalons chaque fois dans les notes.

Les termes vedettes sont donnés dans l'ordre que Viger a adopté, c'est-à-dire que les mots sont classés par lettres, mais ne sont pas rangés dans un ordre alphabétique rigoureux. Un changement dans cet ordre aurait séparé des lexies que l'auteur a réunies dans un même article et sous une seule définition; elles constituent soit des variantes phonétiques ou formelles, comme araignée, arignée ;écolter, escolter; écrapoutir, écrapoutiller; porceline, pourceline; port-épic, porc-épic; soit des équivalents ou des synonymes, comme berline, bordel; menoirs, travail; trompe, bombarde.

Ces groupes de mots sont réunis dans le manuscrit par une accolade que nous avons conservée.

Le signe $\mathbf{x}$ placé devant certains mots vedettes du manuscrit 1 est de Viger et indique que les termes ainsi marqués figurent dans le manuscrit 2; font exception les mots créature, gaspiller et bombarde (sous trompe) qui, bien qu'ils soient marqués, ont été écartés.

Le soulignement est employé par Viger dans le but précis de mettre en valeur le mot vedette, ainsi que les synonymes, les équivalents, les dérivés, les mots de la même famille et même certaines définitions.

Il fait également un grand usage de la majuscule qui joue le même rôle que le soulignement; en revanche, après le point, la première lettre est quelquefois une minuscule, ce qui était fréquent à cette époque, même dans les dictionnaires (v. Académie 1718, notamment).

Chaque fois que l's est redoublé, à l'intérieur d'un mot, l'auteur écrit le premier avec un $s$ long $(\delta)$, suivant en cela une tradition calligraphique qui a été usuelle dans les dictionnaires jusqu'à la fin du XVIII ${ }^{e}$ siècle. Nous avons préféré le remplacer par l's actuel. Nous avons, par ailleurs, conservé la perluète (\&) et l'abréviation du mot et cetera $(\& \mathrm{xc}$.); ces signes qui sont généralisés dans les manuscrits l'étaient également dans les dictionnaires des $\mathrm{XVII}^{\mathrm{e}}$ et $\mathrm{XVIII}{ }^{\mathrm{e}}$ siècles. Les abréviations ont été résolues. La résolution est en italique.

L'orthographe de Viger, qui écrit au début du XIX ${ }^{\mathrm{e}}$ siècle, est encore dans un certain nombre de mots ou de groupes de mots celle des XVII et 
XVIII ${ }^{e}$ siècles. Nous avons conservé cette orthographe ancienne en raison des précieuses informations qu'elle apporte sur les habitudes orthographiques de cette époque.

La graphie ancienne qui revient le plus souvent dans les manuscrits est celle de oi pour ai dans des mots comme anglois, françois, foible, monnoie, roide, etc., et dans les désinences verbales de l'imparfait et du conditionnel comme dans pourroit, faisoit, etc. La graphie ai n'a été admise dans les dictionnaires français qu'au début du XIX ${ }^{\mathrm{e}}$ siècle; la variante foible, notamment, est encore consignée dans Littré.

La chute du $t$ ou du $d$ au pluriel dans les mots en ant et ent représente une autre graphie usuelle à l'époque de Viger. Citons dens, enfans, habitans, instrumens, piquans, etc. Les exemples sont nombreux et généralisés chez l'auteur, bien que l'on trouve de temps à autre l'orthographe actuelle dans enfants et babitants, entre autres. L'hésitation entre le maintien ou l'abolition du $t$ et du $d$ avait cours depuis le XVI siècle : "Etienne Dolet est l'un des premiers à restituer systématiquement dans les formes du pluriel ces consonnes finales muettes devant sn (LarOrth 78); il n'empêche qu'il faudra attendre Académie 1835 pour que la même forme soit retenue pour le singulier et le pluriel.

Enfin, un certain nombre de graphies étaient en concurrence aux $\mathrm{XVII}^{\mathrm{e}}$ et XVIII $\mathrm{X}^{\mathrm{e}}$ sìcles; ainsi cens et sens, guère et guères, byver et hiver, phiole et fiole, orthographe et ortographe, sinonime et synonime, style et stile, ustensile et ustencile, etc.

La graphie du mot temps, que Viger orthographie temps ou tems, était encore fluctuante à la fin du XVIII e siècle. Féraud écrit dans son Dictionnaire critique de la langue française de 1787 : «Temps ou tems, s.m. Le $1^{\text {er }}$ est plus conforme à l'étymologie : le $2 \mathrm{~d}$ comence pourtant à prévaloir : mais lors même qu'on écrit temps, on ne prononce pas le p.»

Certaines graphies étymologiques comme bled (Viger écrit également $b l e ́)$ et $n u d$ sont encore courantes au XVII ${ }^{\mathrm{e}}$ siècle.

On trouve également dans les manuscrits un grand nombre de mots qui comportent un redoublement de consonnes qui sont simples de nos jours comme, par exemple appeller, appercevoir, applanir, fripponner, jetter; et l'inverse, bute, canelé, charetier, désapointé, etc. Les lexicographes ne s'entendaient pas sur ces graphies. Par exemple, Trévoux met deux $p$ à friper alors qu'Académie n'en met qu'un. Féraud notamment relève de très nombreux cas qui témoignent de l'incertitude qui régnait aux $\mathrm{XVII}^{\mathrm{e}}$ et $\mathrm{XVIII}^{\mathrm{e}}$ s. à ce sujet.

Les signes orthographiques : La graphie qui s'écarte le plus de l'orthographe actuelle dans le texte de Viger est celle qui a trait aux accents. 
Nous les avons reproduits fidèlement en raison de l'intérêt qu'ils présentent pour la prononciation. Il n'y avait pas d'accent en latin et le français a conservé longtemps cette tradition, ce qui explique la grande confusion qui existait encore dans ce domaine à l'époque de Viger. "Il faudra attendre Académie 1740 et 1762 pour que soient définitivement adoptés, en français, les usages du système d'accentuation actuel, dont les bases étaient déjà en place en 1550, soit deux siècles auparavant» (LarOrth 1127). Viger n'échappe pas à cette influence, et c'est probablement ce qui dérange le plus le lecteur. Nous avons été très attentive à reproduire fidèlement les accents; lorsqu'ils sont absents, et c'est fréquemment le cas, nous n'avons pas cherché à les rajouter afin de donner une idée exacte de la façon dont on écrivait.

L'accent est souvent absent dans des mots tels que melasse, secher, secretaire, peter, ebranlé, etc.

Ailleurs, l'accent grave est remplacé par l'accent aigu dans les mots caléche, espéce, piéce, siége, mécbe, séche, etc.

L'accent circonflexe est présent dans des termes qui n'en comportent pas, comme dans crême, guerêt, mêts, et inversement dans gout, maitre, ragout, traine, traineau, etc.

Le mot poëte que Viger écrit avec un tréma, a été orthographié ainsi jusqu’à Académie 1878.

Enfin, nous avons utilisé le mot [sic] pour signaler des erreurs d'orthographe et de grammaire ou des graphies aberrantes qui peuvent être le reflet de la prononciation.

\section{Liste des termes, signes conventionnels et abréviations}

[ ]

$\ll \gg$

$\sim$

$£$

\#

8

a. ou act. indiquent une intervention de l'éditeur.

servent à présenter soit un sens, soit une citation.

remplace un mot ou des mots qu'on ne veut pas répéter.

livre anglaise (unité monétaire).

livre (unité de masse).

signe qui a été utilisé par les auteurs du $X V \mathrm{XI}^{\mathrm{e}}$ siècle pour représenter le son [u] dans l'écriture des langues amérindiennes. 
absolt

abstrait

adj.

adv.

anciennt

angl.

antiphrase

app.

archaïsme

augm.

bibliogr.

c.-̀̀-d.

cf.

concret

corr.

de

dém.

dialectal

dialectalisme

éd.

ex.

fam. absolument (en construction absolue : sans le complément attendu).

qualifie un sens (s'oppose à concret).

adjectif.

adverbe; adverbial.

anciennement (présente un mot ou un sens courant qui désigne une chose du passé disparue). Ne pas confondre avec vieux, avec hist.

anglais.

par antiphrase : en exprimant par l'ironie l'opposé de ce que l'on veut dire.

appendice.

mot, expression, tour ancien qu'on emploie alors qu'il n'est plus en usage.

augmenté.

bibliographie.

c'est-à-dire.

confer «comparez» : sert à présenter un mot de sens différent, mais comparable; une expression, un terme de formation semblable.

qualifie un sens (s'oppose à abstrait).

corrigé.

précédant une forme, dans une étymologie, signifie que le mot est formé par dérivation ou composition à partir de cette forme.

démonstratif.

qualifie un mot ou un emploi provenant d'un dialecte, d'un patois, qui n'est pas employé comme un mot du français général.

mot, expression qui provient d'un dialecte ou d'un patois. édité, édition.

exemple (par ex. : par exemple).

familier (usage parlé et même écrit de la langue quoti- 
dienne : conversation, etc., mais ne s'emploierait pas dans les circonstances solennelles.

f. ou fém. féminin.

fig.

figuré : sens issu d'une image (valeur abstraite correspondant à un sens concret).

hist. terme d'histoire, historique.

ib. ou ibid. ibidem (dans le même ouvrage, dans le même passage d'un ouvrage déjà cité).

id. idem (s'emploie généralement pour éviter la répétition d'un nom dans une énumération, une liste).

innovation création d'un mot, d'un sens.

lat. latin.

m., mas., masc. masculin.

Ms., ms. manuscrit.

n. note; neutre.

$n^{\circ} \quad$ numéro.

nouv. nouveau.

p. page.

part. participe.

pl., plur. pluriel.

pop. $\quad$ populaire : qualifie un mot ou un sens courant dans la langue parlée des milieux populaires.

pron. pronom.

publ. publié.

qqch. quelque chose.

qqn quelqu'un.

r. réfléchi.

réimpr. réimpression.

rem. remarque.

rev. revu.

s. siècle. 
s., sub. ou subst. substantif.

sing.

singulier.

spécialt

spécialement.

t.

tome.

V.

verbe; voir.

vieilli

mot, sens ou expression encore compréhensible de nos jours, mais qui ne s'emploie plus naturellement dans la langue parlée courante.

vol.

volume.

$\mathrm{vx}$

vieux (mot, sens ou emploi de l'ancienne langue, incompréhensible ou peu compréhensible de nos jours et jamais employé, sauf par effet de style : archaïsme). 


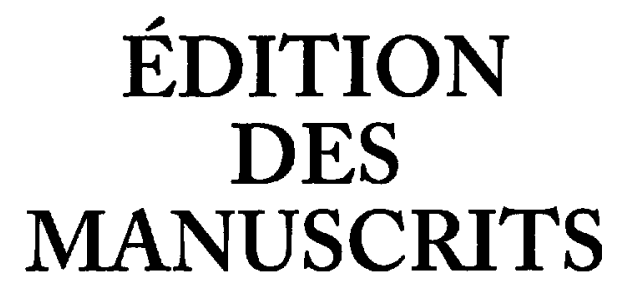


12. Naventins 1810.

\section{Fo, Verrean 67 No}

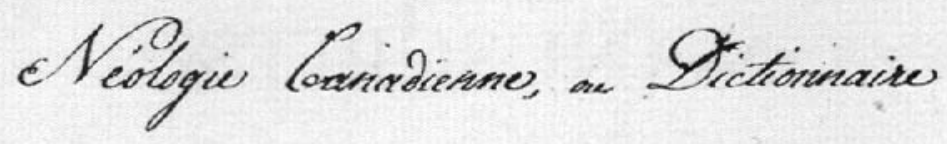

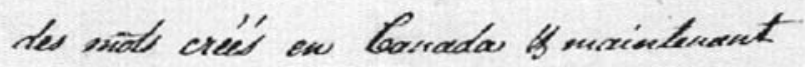

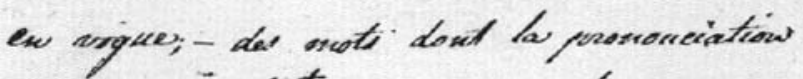

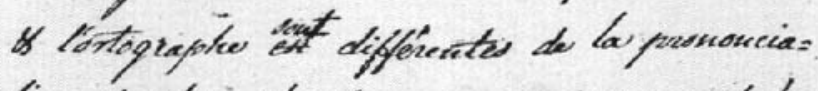

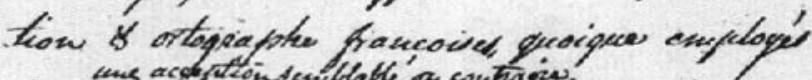

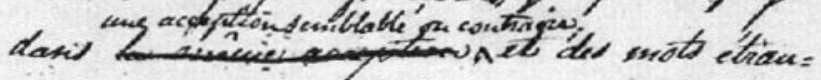

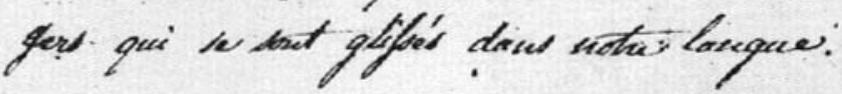

qaequear Diger

Page titre de la Néologie canadienne de Jacques Viger, datée du 12 novembre 1810. Manuscrit conservé au Musée de la civilisation, fonds d'archives du Séminaire de Québec. 


\title{
[MANUSCRIT 1]
}

12. Novembre, 1810.

\section{Néologie Canadienne,}

\author{
ou \\ Dictionnaire
}

des mots créés en Canada $\&$ maintenant en vogue ; - des mots dont la prononciation $\&$ l'ortographe sont ${ }^{1}$ différentes de la prononcia $\approx$ tion \& ortographe françoises, quoique employés dans une acception semblable ou contraire ${ }^{2}$; et des mots étrangers qui se sont glissés dans notre langue. 
[CAHIER A]

Appointer. V. act. Ce verbe qui signifie en bon françois - donner des appointe mens, c'est-à-dire, une pension ou des gages à quelqu'un, signifie ici nom $\approx$ mer à une place : Il a plu à Monsieur le Gouverneur d'appointer Monsieur... Grand Voyer du district. Anglois.

Appointement. sub. mas. (pension, gages.) Ce mot est employé ici pour emploi, charges, place. Monsieur D. risque par sa conduite de perdre ses appoin temens. Anglois.

Amarrer. V. act. (terme de marine) A Québec surtout ce mot est employé à tout moment, et dans tout ce qu'il faut lier ou attacher. Ainsi un domes $\approx$ tique amarre les courroies des souliers de son maitre; on amarre une guenille autour d'un doigt blessé.

$x$ Amancher pour Emmancher, V. act. (mettre un manche). Outre sa signification propre, on se sert encore d'aman cher pour raccommoder (Voyer ${ }^{(3)}$ raman $\approx$ cher): Il a bien amanché ce ciseau. Il veut dire aussi refaire : mon lit est ${ }^{(4)}$ défait, vous direz à la fille de l'amancher ou le ${ }^{(5)}$ ramancher. ${ }^{(6)}$

Avisse. sub. fém. - pour vis que l'on pros nonce visse; piéce ronde de fer ou de bois canelée en ligne spirale, et qui entre dans un écrou.

$x$ Allumer. v. a. Quand on ne joint point de régime à ce verbe, il est toujours sous entendu que c'est de la pipe dont on veut parler. Ex : Entrons allumer; veux-tu allumer ${ }^{()}$; quand 
tu auras allumé, tu me donneras du feu. Il signifie aussi se reposer, comme dans ces phrases : mes en fans, dira un maitre à ses employés, quand vous aurez fini cette partie de votre ouvrage, je vous permêts ${ }^{(8)}$ d'allumer, parce qu'en effet ces mo $\approx$ ments de repos sont mis à profit par les fumeurs. Un habitant dira à un de ses amis : si tu passes dans ma paroisse, arrête ${ }^{(9)}$ allumer chez moi. Quand je serai au bout de ce guerêt, j’allumerai.

Argent. sub. mas. Comme métal et comme monnoie, argent est toujours masculin. ainsi l'on doit dire : argent poli, faux argent, ${ }^{(10)}$ bon argent, et non pas polie, fausse, bonne.

$x$ Amidon. sub. mas. Certaine pâte qui est faite de fleur de froment sèche, $\&$ qu'on délaie pour en faire de l'em $\approx$ pois. L'Amidon n'est pas générale ment connu sous ce nom, mais sous celui d'empois, et il se vend et se débite sous ce nom.

Amont la côte. Manière de parler plus particulière au district de Québec. Aller amont la côte, grimper \&c.

Âbre, sub. mas. - pour Arbre. faute généralement commise par les habitans. $x$ Ahurissant, te. ${ }^{(11)}$ adj. ${ }^{(12)}$ du verbe françois ahurir, qui veut dire, interdire, éton $\approx$ ner, rendre stupéfait. Il signi[fi]e ici ennuyant. cet homme est ahurissant. C'est une chose ahurissante. Il est quelque ${ }^{(13)}$ fois substantif. finis donc, ahurissant.

x Abât. sub. mas. Mot employé comme celui de bordée, en hiver, pour expriz mer une grande quantité de neige. 
Nous ${ }^{(14)}$ aurons certainement sous peu un amât ${ }^{(15)}$ de neige. Voyez Bordée. Araignée, \} sub. mas. pour Araignée, s. fém. Arignée - $\}$ On employe ${ }^{(16)}$ le plus ordinairement le mot d'araignée ou d'Arignée au mas. Un gros arignée, pour une grosse araignée.

$x$ Apichimon. sub. mas. Espèce de bourlet [sic] de vieux linges, ou guenilles que les habitans mettent sur le cou du bœuf qui labourre, pour le lui garantir du mal que ${ }^{(17)}$ pourroit faire le joug. Ils appellent aussi apichimon, un morceau d'étoffe, ou une petite peau de mouton dont ils se servent en guise de selle, lorsqu'ils montent à cheval. On l'emploie aussi pour méchant lit, grabat.

Allemand. Nom de nation dont on se sert dans cette phrase : c'est une tête d'Allemand, il a une tête d'Allemand, pour, qu'il est entêté, opiniâtre.

[CAHIER B]

Affaire. - avoir affaire de, Ex. j'ai affaire de vous, entrez donc. pour j'ai affaire à vous, \&c. ou j'ai besoin de vous.

$x$ Arse. sub. Ce substantif s'emploie dans ${ }^{(18)}$ cette phrase : cette chambre est $\mathrm{si}$ petite qu'on n'a pas l'arse de se remuer, pour la facilité, le moyen. J'aime à avoir de l'arse partout où je suis, pour n'être pas gêné. Il n'y a pas dans ce lit l'arse de se retourner, ou assez d'arse pour y coucher deux, pour il n'est pas assez large, il n'y a pas l'espace de...

à même - boire à même - vous

êtes à même de faire cela, d'aller là, si vous voulez je l'ai mis à même ${ }^{(19)}$. acquet - vous avez plus d'acquet de vous arranger que de plaider ${ }^{(20)}$. 
Attisée ${ }^{(21)}$. sub. fém: du verbe attiser. ce mot ne se dit pas. faites une bonne attisée pour la nuit. -

Achigan - sub. mas. (poisson).

\section{B.}

[CAHIER A]

Bourgogner. v. act. - Battre d'une manière honteuse, battre à plates coutures. Je l'ai Bourgogné.

On le fait préc[éd]er aussi du Verbe Faire \& l'on dit : il s'est fait bourgogner, pour exprimer qu'il a été battu d'une manière deshonorante. Ce verbe doit sa naissance à la défaite du Général Burgoyne, que les Canaz diens nomment encore Bourgogne.

$x$ Bombe. sub. fém. mot usité plus particu lièrement dans le District de Québec, au lieu de celui de Bouilloire, sub. fém, ou le vase dans lequel on fait bouillir l'eau pour le thé. Dans le District de Montréal, on employe dans la même signification le mot Canard. Ex. Mettez la bombe au feu : apportez le ${ }^{(2)}$ canard.

$x$ Boucane, Sub. fém. - pour fumée. Quelle bou cane il y a ici!

$x$ Boucaner. V. n. pour fumer. La cheminée de cette maison boucane trop pour l'habiter; à un fumeur, ne boucane donc pas tant !

Berline - sub. fém. Sortes de voitures Bordel - sub. mas. d'hyver pour la com $\approx$ modité des voyages. Ce sont des voitures plus grandes et plus pé santes [sic] que les carioles. Le Bordel est la plus grossière de ces voitures \& ne se voit que dans la Campagne, chez les habitans ${ }^{(2))}$ pauvres. 
x Berlancille. sub. fém. pour Balançoire, Escarpolette ${ }^{(24)}$, ou Brandilloire. Jeu d'enfant ; corde, branche d'arbre ou planche avec quoi on se balance on se brandille.

$\mathbf{x}$ Berlanciller. v. n. pour se balancer, ou se brandiller. v. r. viens berlanciller. Les enfans ${ }^{(25)}$ ont berlancillé ${ }^{(26)}$ tout le jour.

Bombarde. Sub. fém. Voyez Trompe.

Bouquin. sub. mas. C'est ou une plume teinte, ou un petit cilindre, percé aux extrémités, soit d'étain, de fer, de cuivre ou d'argent, ou même un os, dont ${ }^{(27)}$ nos habitans se servent pour orner leurs pipes, ou en alon $\approx$ ger ce qu'ils appellent le manche.

Balier. V. a. au lieu de Balayer. Ex. dites à la fille de balier la chambre.

Boyard. sub. mas. pour brancard (civière)(28). sub. mas. Ex : porter quelqu'un sur un boyard; trans $\approx$ porter des pierres sur un boyard.

Brancard. sub. mas. On n'emploie ce mot que pour la calèche ${ }^{(29)}$; le brancard de la calèche, pour les autres voitures à brancard, voyez les mots travail \& menoirs.

$\mathbf{x}$ Butin, sub. mas. sous ce mot on entend quelquefois les effets, meubles $\&$ hardes d'une personne quelconque. Il s'est échappé et a emporté tout son butin. C'est mon butin. (Gaspiller) ${ }^{(30)}$

Bourguignon. sub. mas. On appelle ainsi les gros glaçons que l'on ${ }^{(31)}$ apperçoit soulevés à la surface de la rivière, lorsqu'elle est prise. Que de bourguig $\approx$ nons sur la rivière ! Ce bourguignon est bien transparant [sic] !

$\mathbf{x}$ Bordée, sub. fém. Une bordée de neige; nous avons essuyé une furieuse bordée de neige ${ }^{(32)}$, ou absolument, une grande bordée. C'est a dire qu'il est tombé beaucoup de neige. On emploie aussi le mot $\underline{\text { Abât. }}$. 
Bèr ${ }^{(33)}$, sub. mas. pour Berceau (le $\mathrm{l}^{(34)}$ petit lit où l'on couche les enfans à la mammelle.)

Berloque sub. fém. On appelle ainsi une montre sujette à aller mal. C'est une berloque.

$x$ Bicler. V. n. au lieu de Loucher. V. $n$.

Il bicle, elle a le malheur de bicler.

$x$ Bicleux. se. adj. pour Louche, adj. -

Il est bicleux, sa sœur est bicleuse.

Il est aussi sub. c'est un bicleux, une bicleuse.

Banal. sub. mas. Il se dit exclusivement d'un taureau, où est le banal? Je vais couper mon banal; et d'un homme extrêmement fort $\&$ de grande taille, bon Dieu! quel banal!

Bougon. sub. mas. On dit d'un petit homme, c'est un bougon; un bougon de chandelle, pour un petit bout; on nomme ${ }^{(35)}$ aussi ${ }^{(36)}$ une pipe bien courte, un bougon, un bougon de pipe. (37)

Buffet. sub. mas. On l'emploie quelquefois au lieu de bureau. (espèce de table à plusieurs tiroirs et tablettes, où l'on enferme des papiers, et sur laquelle on écrit[)].

[CAHIER B]

Bord. sub. mas. on l'emploie pour le versò d'un livre. vous trouverez ce mot de l'autre bord. ${ }^{(38)}$ $2^{\circ}$ côté, - regardez l'autre bord de cette médaille; cette indienne est aussi bien empreinte d'un bord que de l'autre.

Batailleur, se. sub. pour sujet, enclin à $^{(39)}$ se battre. c'est un batailleur de profes $\approx$ sion. Il est le plus grand batailleur qui existe.

Bandon. sub. fém. Saison de l'année où il est permis et d'usage de laisser aller les animaux par toute la campagne. Ex. La Bandon commence au mois de... $\&$ finit au mois de... Les animaux ont bandon, de ce jour. Don $\approx$ ner bandon aux animaux, pour dire les laisser partir pour courir les champs. 
Biner. v. n. On l'employe pour exprimer qu'une personne $\underline{a}^{(40)} \mathrm{du}$ dépit, qu'elle ${ }^{(41)}$ enrage. Il bine; on l'a fait biner. Voyez corner.

Brouscailler. V. a. Maltraiter ${ }^{(4)}$ de paroles ou autrement ${ }^{(43)}$. c'est ${ }^{(44)}$ le précurseur de battre.

Ne me brouscaille pas tant. Il l'a bien brouscaillé ; après s'être longtems brouscaillés, ils en sont venus aux mains. ${ }^{(45)}$

Bourasser. v. a. s'emploie pour gourmander; gronder continuellement. Si je lui parle, il va me bourasser. Il n'a fait que bourasser tout le jour. pourquoi me bourasser, je ne vous dit [sic] rien. Bourasseur ${ }^{(46)}$ - se. sub. c'est un bourasseur, une bourasseuse : c-a-d. qu'ils grondent sur tout; qu'ils ont l'humeur à la gron $\approx$ derie, d'une humeur hargneuse.

Bourasse. sub. fém. Mauvaise humeur.

j'ai éprouvé toute sa bourasse; il est aujourd'hui d'une bourasse peu com $\approx$ mune. sa bourasse est passée.

Allez passer votre bourasse ailleurs. Il a passé sa bourasse sur ce pauvre chien, qui ne lui faisoit rien, pour dire qu'il a maltraité son chien par mauvaise humeur. ${ }^{(47)}$

Basque. sub. mas. Nom de Nation dont on [se] sert dans cette façon de parler, malin comme un Basque; c'est un malin Basque. Le Dictionnaire de l'Académie dit Aller comme un Basque, courir comme un Basque, pour dire aller fort vite, courir fort vite.

$x$ Brâsser. V. a. (remuer avec les bras, à force de bras. - Brasser de l'or, de l'argent fondu) on dit ici, brassez \& remuez ${ }^{(48)}$ le poële, pour le feu du poële; brassez la poële, le chaudron, pour, ce qui est dedans. \&c. on dit aussi : je l'ai brassé d'importance, de la même manière que l'on dit en françois; je l'ai bien savonné, (réprimendé) [sic]. 
Baiser. V. a. On dit en style badin; il a été baisé dans telle occasion, pour retapé $^{(49)}$. On se sert aussi de ce dernier. Il s'est fait baiser ${ }^{(50)}$ en pincettes, il l'a baisé en pincettes en guedou ${ }^{(51)}$. Baiser en pincettes est françois, pour exprimer l'action de prendre doucement les deux joues de celui qu'on baise. C'est une des caresses auxquelles on accoutume les enfans; baisez-moi en pincettes.

Bredas ${ }^{(52)}$. sub. mas. On l'emploie ici pour

remue-ménage, dont on se sert aussi quel brédas! On appelle faire le bredas, faire les gros ouvrages de l'intérieur de la maison, la lessive, le blanchissage, le lavage du linge, des meubles \&c. On dit aux enfans ou autres personnes qui par leurs jeux, par nécessité, par faute d'attention ou autrement dérangent les meubles avec bruit ou les entassent avec confusion, quel brédas faites vous! bon dieu quel bredas ${ }^{(53) !}$ (confusion.).

Bredasser ${ }^{(54)}$. V. n. c'est s'occuper à des ouvrages de ménage inutiles. Il a bredas $\approx$ $\underline{\text { sé }}^{(55)}$ toute la journée. Vous ne faites que bredasser, occupez-vous donc de votre lavage. Il veut aussi dire, maltrair ter ${ }^{(56)}$ légèrement ${ }^{(57)}$; attends - attends, je vais te bredasser; il l'a bredassé (c'est peut-être tracasser.)

Bredassier, re. sub. - (tracassier, re.)

Bredasserie, sub. fém. (tracasserie.)

Braye. sub. fém. Instrument dont on se sert $\grave{a}^{(58)}$ brayer le lin, le chanvre.

Brayer. V. a. brayer du lin, du chanvre; ${ }^{(59)} \mathrm{en}$ séparer les filets de la partie ligneuse.

Belt, sub. fém. mot anglois en vogue au lieu du françois - Baudrier. sub. mas. Avez-vous nétoyé $\left[\right.$ sic] $\mathrm{ma}^{(60)}$ belt, pour mon baudrier.

Bougon. sub. mas. mot qu'on emploie par

dérision, ou mépris, dans le [même] sens que celui 
de bout d'homme, en france. C'est un petit bougon, pauvre bougon, je ne vou $\approx$ drois pas te frapper. Quelque ${ }^{(61)}$ fois on dit Nijon. ${ }^{(62)}$

Boudin. sub. mas. on l'emploie dans cette phrase :

faire du boudin, il fait du boudin, pour dire d'une personne qu'elle boude.

$x$ Beurrer. V. a. Il n'est pas françois, on l'emploie pour étendre du beurre sur... beurrez le pain. Voyez plus loin. ${ }^{63)}$

Beurrer V.a.

beurrez ce plat, beurrez-vous les mains, avant que d'y mettre, ou de prendre telle chose; beurrez-lui du pain pour sa collation. On dit même, beurrer ${ }^{(64)}$ de la graisse, des confitures, de la crême \&x.

$x$ Beurrée. sub. fém. Ce nom qui ne doit s'appliquer qu'à une tranche de pain, sur laquelle on a étendu du beurre, se donne ici, encore à toute tranche de pain sur laquelle on a étendu de

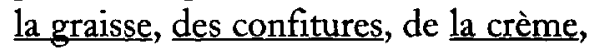
de la melasse, ou autre substance grasse, onctueuse ou liquide. $\underline{\text { Beurrée }}$ de graisse, de sirop, de crème \&c.

Blonde. sub. fém. pour Amante. On donne pour blonde à $N$. Mademoiselle $\mathrm{O}$. est-ce vrai?

Il est allé voir sa blonde. c'est un amoureux de 36 blondes, il est fort sur la blonde. il a une jolie blonde.

Bavasser. V. n. Il ne se dit guères que d'une personne ivre; et l'on dit : laissons-là [sic], elle ne fait plus que bavasser, pour exprimer en même tems qu'elle parle beaucoup, confusé ment, sans savoir ce qu'elle dit, en prononçant imparfaitement $\&$ artiঞ culant avec peine. c'est, je crois, bavarder $\&$ balbutier. ${ }^{(65)}$

Beauté. sub. fém. On emploie très souvent ce mot d'une manière adverbiale - 
pour exprimer beaucoup; il y en a une beauté. Ils sont une beauté de spec tateurs, pour un grand nombre. \& ironi quement pour peu, petit nombre, oui, je crois qu'il y aura une beauté de monde ce soir, au bal ${ }^{(66) !}$ !l y en avoit une beauté!

faire Brun. V. n. On dit en parlant de l'obscurité de la nuit : il fait bien brun, il faisoit un peu brun. Cette expression paroitroit venir des marins, qui disent le brun de la nuit, qui se dit sur mer dans le même sens. Le tems est brun pour sombre, est francois.

Brunante. sub. m. pour la Brune (le tems entre le soleil couché et la nuit.) A la brunante pour sur la brune, c-à-d. sur le soir, entre chien et loup. toutes ces expressions que nous employons sont bonnes, à l'exception de la première.

Bagage - sub. mas. pour Ménage.

Brillant - Proverbe - il prend du café comme la Brillant.

Bleuet. sub. mas. - (plante). Voyez différence entre celui du Canada \& celui d'Europe.

\section{C.}

[CAHIER A]

x Canard. sub. mas. mot usité plus parti culièrement dans le district de

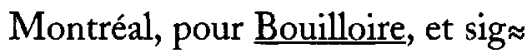
nifiant exclusivement le vase qui sert à bouillir l'eau pour le thé. Dans le District de Québec on se sert du mot Bombe. Ex. Rem $\approx$ plissez d'eau le Canard : ôtez la bombe du feu.

$x$ Cordeau, sub. mas. mots employés le plus ${ }^{(67)}$ Courroie, sub. fém. $\int$ communément au pluriel à la place du mot propre Guide, usité 
avec justesse à Montréal, pour exprimer les longues rênes attaz chées à la bride d'un cheval attelé, et pour le conduire. On doit donc dire : donnez moi les guides, et non les cordeaux ou les courroies.

Coup - Voyer ${ }^{(68)}$ Faire coup.

$x$ Couette. sub. mas. (lit de plume). Ce mot n'est point connue $[$ sic] ici dans cette acception. Couette signifie ici la queue de cheveux que porte [sic] les hommes. Faites-moi la couette, c'est ${ }^{(69)}$ dire, entourrez ce ruban autour de ma ${ }^{(0)}$ queue de cheveux.

Cariole. Sub. fém. Voiture d'hyver des villes et des campagnes, pour le plaisir de la promenade ou l'utilité des voyages. C'est une voiture élégante \& peinturée. C'est le traîneau en bon françois.

Charger. V. n. Employé parmi les habia tans sans régime, pour dire mettre le tabac dans la pipe. Ex : quand tu aura [sic] chargé, donne $e^{(71)}$ moi le tabac.

Cabrouet. sub. mas. Voiture $\grave{a}^{(72)}$ deux roues, longue et étroite dont nos charetiers se servent.

$x$ Cadre. sub. mas. (bordure de tableau). On nomme ${ }^{(73)}$ Cadre le tableau même. Voilà un beau cadre, pour : voilà une belle peinture ou tableau.

Chardron, pour Chardon, sub. mas. Plante. Caribou, pour, le Renne, animal sauvage. Castonade, pour Cassonade, sub. fém. Sorte de sucre. -

Cahot, sub. mas. (espéce de saut que fait une voiture, en roulant sur un chemin raboteux $\&$ mal uni.) Dans cette acception on dit, faire des cahots, 
éprouver des cahots, nous avons trouvé des chemins qui font faire bien des cahots. Le cahot est toute autre chose que cela ici. On dit : il y a bien des cahots sur ce chemin, pour dire qu'il est beaucoup ${ }^{(74)}$ rabo $\approx$ teux et il n'est employé que pour les chemins d'hyver. Monsieur l'Inspec teur a donné ordre d'abattre les cahots, c-à-d. d'applanir le chemin en coupant les petites butes de neige. (75)

Couper pour couper les bleds, au lieu de scier les bleds, ce qui se fait à l'aide de la faucille. (76)

Crocheter, v. a. (qui dans son acception propre veut dire, ouvrir une porte \&c. avec un crochet, avec in $\approx$ tention de voler, ou à quelque autre mauvais dessein, mais que nous n'employons pas dans cette signifi $\approx$ cation[)]. On ne se sert ici(7) de crocheter que dans cette phrase : crocheter des pois, c.-à-d. les couper ${ }^{(78)}$ avec un petit crochet au bout d'un bâton. Tous mes pois sont crochetés. \&c.

Crocheteur, sub. mas. Celui qui crochette [sic] des pois.

Couronel, sub. mas. - pour Colonel.

Corporal, sub. mas. très usité pour Caporal.

Cartron, sub. mas. pour Carton.

$x$ Côte. sub. fém. Ce mot signifie le $\mathrm{e}^{(7))}$

penchant d'une montagne, $\&$ d'une colline \& les ${ }^{(80)}$ rivages de la mer : Côte d'une telle montagne, les Côtes de l'Océan. Mais on l'applique à tort ici à toute éminence, hauteur ou élévation.

Cheniquer. V. n. craindre de se faire battre. $\mathrm{Tu}$ as cheniqué, un tel t'a fait cheniquer. Il emporte avec lui la honte $\&$ le déshonneur. 
Cheniqueur, euse. sub. qui chenique.

Casseau. sub. mas. Sorte de petit meuble d'écorce ou de bois, pour mettre des fruits, ou autres choses.

[CAHIER B]

x Comfortable. adj. cet adjectif a beaucoup de significations. Il est employé pour $1^{\circ}$ consolant : c'est une nouvelle com $[\approx]$ fortable. $2^{\circ}$ agréable, tems, jour com $[\approx]$ fortable. $3^{\circ}$ doux, content, mener une vie comfortable. $4^{\circ}$ qui réjouit, qui fait plaisir, une liqueur comfortable. $5^{\circ}$ qui fortifie, confortatif, une nourria ture, un mett $[s i c]$ comfortable. Anglois.

$x$ Capuche. sub. fém. Ce mot est quelque ${ }^{(22)}$ fois usité dans la campagne au lieu de chapeau de femme : une capuche de paille, de castor; apporte à ta mère sa capuche; où est la capuche de ta sœur?

Corner. V. n. employé comme $\underline{B \text { iner, }}$ pour exprimer qu'une personne, enrage, éprouve un dépit, un déplaisir grand et sensible. Il a corné comme il faut. c'est trop corner. ce contretems le fera corner. Voyez Biner. \& Ebrayer ${ }^{(83)}$

$x$ Chienneter. V. n. pour chienner, (faire des chiens.) ma chienne a chienneté, pour chienné.

$x$ Cree, nom d'une nation Sauvage du Canada, dont on se sert dans cette phrase; malin comme un Cree. prononcez $\mathrm{Cris}^{(84)}$.

Casque. sub. mas. On donne ce nom à un bonnet de fourrure dont [on] se sert ici en hyver.

Crible. sub. mas. pour train de bois. ${ }^{(85)}$

Cage - \& Cajeux -(86)

Cajeux. sub. mas.

Cage - sub. fém. $\}$ pour trains de bois flottés ${ }^{(87)}$

Crible - sub. mas.) 
x Côte. sub. mas. [sic] On se sert aussi de ce mot

pour marquer une rangée de terres concédées, ou d'habitans de la campagne.

La Côte de la visitation, c'est un habitant de la côte Saint Luc; je viens de la grand' côte. \{on dit : courir les côtes pour les campagnes ${ }^{(88)}{ }^{(89)}$

$x$ Chatoner. V. n. qui se dit ici d'une chatte qui fait ses petits, - au lieu de chatter qui est le verbe françois. La chatte est au moment de chatoner, pour de chatter; elle a chatoné cette nuit, pour chatté.

$x$ Cavalier. sub. mas. pour Amant. Le cavalier de Mademoiselle P. est Monsieur V. - cette petite parle ${ }^{(90)}$ déjà de cavaliers, elle en désire un.

$x$ Cantine. sub. fém. (petit coffre divisé par compartimens, pour porter des bouteilles et des phioles en voyage.) On lui donne ici ce nom et celui de cannevette, qui n'est pas françois.

$x$ Cantine. sub. fém. On emploie indiffére[m]ment ici ce mot pour celui de cabaret ou taverne ${ }^{(91)}$. Ce mot nous vient de l'armée. (La cantine est, à l'armée, le lieu où l'on vend du vin et de la bière aux soldats, sans payer aucun droit.) Viens à la cantine.

$x$ Cantinier. re. sub. mas. Cantinier, en françois, est celui qui tient la cantine à l'armée ${ }^{(92)}$. Nos Canadiens donnent ce nom à tout cabaretier ou tavernier ${ }^{(93)}$ ou détailleur de boissons fortes. Le cantinier n'est pas à la maison, mais nous y trouve $\approx$ rons la cantinière. ce dernier mot ne se dit pas en françois. - c'est cabaretier ou tavernier (94).

$x$ Canoter. v. n. ${ }^{(5)}$ conduire un

canot. il se dit des enfans qui s'amusent à se promener ${ }^{(96)}$ en canot à peu de distance du rivage $\&$ c. ils ont canoté, tout le jour; l'enfant est à canoter. \&c. Viens canoter. 
$\mathbf{x}$ Canoteur, euse. sub. qui aime ou qui sait bien canoter. Il ne se dit guère que des enfans.

$x$ Canotée. sub. fém. Autant qu'un canot peut contenir ${ }^{(97)}$ de marchandises ou autres choses. Il a passé une canotée d'enfans. Je viens d'acheter une canotée de volailles. une canotée de marchan[ $[\approx]$ dises. On appelle aussi canotée, la charge d'un canot ${ }^{(98)}$, j'ai monté à Michillimakinac, avec 6 canotées, ( $\&$ aussi 6 canots.)

Coppre. sub. fém. pour Sou s. m. (monnoie de compte, la $20^{\mathrm{e}}$ partie de la livre, valant 12 deniers.) Une coppre, dix ou douze coppres pour un sou, dix ou douze sous.

Coton. sub. mas. c'est le nom que l'on donne à l'épi de bled d'Inde, lorsqu'on en a mangé ou ôté les grains. ramassez le[s] cotons de bled-d'Inde et les donnez à la vache on dit proverbialement : sec comme un coton de bled d'Inde. - Et il a eu un beau bled d'inde, ou il lui a donné un beau bled d'inde ;(99) pour un pied de nez.

Clairon. sub. mas. On l'emploie aussi pour tirans. Voyez ce mot.

Chandelle - Il a évité une belle chandelle pour un grand malheur, péril. ${ }^{(100)}$

$x$ Créature. sub. fém: pour femme, comme femelle de l'homme. Ex : il y avoit là de jolies créatures, beaucoup de créatures. On dit aussi : il va voir, il est allé voir la créature, il fré quente la créature. autres sens.

Confessionnals. sub. mas. plur. de confession $\approx$ nal, employé généralement au lieu de confessionnaux. Il y a foule aux confessionnals, de ce tems-ci.

Civière - que nous nommons - Boyard. 


\section{D.}

[CAHIER A]

x Désapointer - v. act. - vieux verbe qui

étoit employé dans ce sens : ôter les

appointemens à un homme de guerre.

Ex. On a dé[s]apointé plusieurs officiers

réformés. Il n'est plus d'usage.

Cependant nos ${ }^{(101)}$ aimables lont pour $\approx$

tant ressuscité à l'aide d'un auxi

liaire. Ainsi l'on dit : j'ai été gran $\approx$

dement désapointé aujourd'hui, je n'ai

pas trouvé Madame $D$. chez elle. Je

devois recevoir $£ 100$ hier, mais j'ai

été désappointé, mon débiteur est

enfui. \&c. De sorte qu'un homme a qui l'on a manqué de parole, qui éprouve quelque contretems ou quelque traverse, qui manque son coup, qui ne vient point à bout de son dessein qui est trompé dans son attente, \&c. qui échoue dans ses plans ou ses projets est dans tous ces cas désapointé. Ce mot est Anglois.

$x$ Démancher. V. a. (ôter le manche.) - Outre sa vraie signification, on l'emploie aussi ainsi. J'ai le bras, le doigt démanché, pour démis. J'ai fait démancher mon écurie, pour exprimer ou que je l'ai fait jetter bas, ou que je l'ai fait défaire en parti [sic]. Mon horloge est démanchée, pour dire qu'elle n'est plus règlée, plus en ordre. Sa voiture s'est démanchée, cette chaise est démanchée, pour dire qu'elles sont brisées. J'ai démanché ma robe, pour dire qu'on l'a décousue.

$x$ Débarquer. v. a. \& n. On l'emploie, outre sa signification ordinaire, pour descendre de voiture : Monsieur B. 
est arrivé de sa campagne, il débar quoit de sa caléche comme je passois devant chez lui. Vite, vite, débarquez, on vous attend. Il est débarqué de bateau ${ }^{(102)}$ de cheval. Avez-vous débarqué ma malle?

$x$ Décent - te. adj. On dit : il a eu des funérailles très décentes, pour honorables. Cette maison est bien décente, pour belle ou bien meublée. cet homme est bien décent dans son habillement, pour dire qu'il est vêtu bien proprement. \&c. Anglois.

$x$ Diriger. V. Il est employé avec le verbe Etre, pour exprimer, être enjoint, avoir ordre. Je suis dirigé par son Excellence de vous dire... pour je suis enjoint, j'ai ordre. \&cc.

Deusse. - pour Deux. nombre cardinal. Dram. sub. mas. V. cage - cajeux - crible. Dégelée - sub. fém. Nous employons ce mot ici au lieu de dégel, s. m. (fonte de la glace, de la neige, par l'adoucissement de l'air.) Il y a eu hier une grande dégélée, les érables ont du [sic] couler. On fait aussi dégélée ${ }^{(103)}$ sinonime de volée de coups; il lui a donné une bonne dégelée, pour une bonne volée de coups. Dans cette acception on se sert aussi du mot Ramasse. Voyez Fricasser.

$x$ Dégobillage. sub. mas. pour dégobillis. ${ }^{(104)}$ Dégobiller et dégobillage sont employés ici indifféremment de ${ }^{(105)}$ vomir; $c e \approx$ pendant ils ne se disent que du vin et des viandes qu'on a prises avec excès. dégobiller son diner; cela sent le dégo: billis. (ces mots sont bas.)

x Décaniller. V. n. Se retirer promptement de quelque lieu; ces enfans décanillèrent bon train, quand ils surent que vous veniez à eux. A peine vous vit-on, que toute la maisonnée décanilla. 
On dit faire décaniller quelqu'un d'un lieu, dans le même sens. Si j'étois de vous je ferois décaniller ces gens de votre maison. ${ }^{(106)}$

Décanillé, ée, part. Elle est décanillé [sic] de ce matin, sans m'en prier. \&c.

Déboutonner - V. a. On l'emploie au figuré dans ces phrases : il est difficile de le faire déboutonner, $\mathrm{c}-\grave{\mathrm{a}}-\mathrm{d}$. de le faire expliquer, de lui faire dire sa façon de penser. On a réussi à le faire débou tonner, à savoir sa juste opinion. Il s'est à la fin déboutonné, il a à la fin dit ce qu'il pensoit ${ }^{(107)}$ du sujet. On dit encore, en badinant, las des sottises qu'on débitoit à ses oreilles, ou n'y pouvant plus tenir, notre homme se déboutonna pourtant \& $\&$. pour exprimer qu'il parla d'une manière éloquente \& triomphante.

Drigaille. synonime - Bagage \& Butin.

\section{$\underline{E}$.}

[CAHIER A]

x Embarquer. V. act. \& n. ${ }^{(108)}$ Il est bien commun ${ }^{(109)}$ d'entendre dire ici : ${ }^{(110)}$ la voiture étant à la porte, nous embarquâmes \&c. pour montâmes dedans. Il signifie quelques fois partir en voiture pour voyage. Ainsi, de ${ }^{(111)}$ deux personnes qui sont convenues d'aller faire une promenade, l'un dira à l'autre : soyez prête à embarquer à 2 heures. Embarquer à cheval. On l'emploie aussi pour Mettre dedans : avez-vous embarqué ma cassette dans la voiture?

$x$ Empois. sub. mas. Espèce ${ }^{(112)}$ de colle faite avec de l'amidon, et dont on se 
sert pour rendre le linge plus ferme et plus clair. Ex : emplois [sic] blanc, empois bleu, mettre le linge dans l'empois. On donne aussi le nom d'empois, ici, à l'amidon même, quoiqu'improprement. Voyez Amidon.

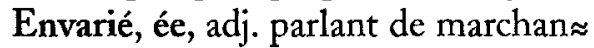
dises gâtées dans un vaisseau. C'est avarié. Etoffes avariées ${ }^{(113)}$.

Espérer - v. a. pour attendre. (espérer une récompence [sic], une succession, la vie éternelle. Il est françois.) mais c'est bien improprement que l'on dit : j'es $\approx$ père une visite aujourd'hui, ou Monsieur un tel; pour dire s'attend à avoir ou recevoir la ${ }^{(114)}$ visite de quelqu'un, ou qu'on attend Monsieur un tel. Dites à cet homme de m'espérer dans cette chambre, (115) espérez-moi ici un instant, ${ }^{(116)}$ je suis à vous dans la minute, pour de m'attendre, attendez-moi, ou (pour se servir du mot même) en l'employant absolument : dites à cet homme d'espérer, espérez un instant, Monsieur va paroitre. - Anglois.

(I have expected you these two hours, je vous ai attendu deux heures; et non pas je vous ai espéré \&c.) -

Ecroi. sub. mas. pour les petits des animaux, \& particulièrement des bêtes à corne[s]. Ma vache a eu un bel écroi. Cette vache est à son premier écroi.

Etage. sub. mas. On emploie ici le mot de premier étage, pour celui de rez-dechaussée. Ainsi l'on dit d'une maison qui a deux rangées de fenêtres, qu'elle est à deux étages. Monsieur M. reste au premier étage de cette maison, pour au rez-de-chaussée; et que le second étage est à louer, pour le premier étage. 
Etau. sub. mas. (sorte de table sur laquelle un boucher expose de la viande en vente.) On le dit au lieu d'étal. Allez à cet étau pour à cet étal. mais au pluriel on dit comme on le doit, des étaux.

Escabeau, sub. mas. (simple siège ${ }^{(117)}$ de bois sans bras ni dossier.) On appelle ainsi un meuble à plusieurs degrés, portatif, dont on se sert dans la maison, pour atteindre à quelque chose d'élevé, en montant dessus.

Epiochon. sub. mas. Epi de blé d'Inde, ou maiis, petit, cassé, ou peu ${ }^{(118)}$ garni de grains.

Ecopeau. s. m. pour Copeau, s. m. (Eclat, morceau de bois que la hache ou quelque autre instrument tranchant font tomber du bois qu'on abat, ou qu'on met en œuvre). gros copeaux, menus copeaux, brûler des copeaux, au lieu d'écopeaux. (sec comme un écopeau.) -

$x$ Ecœurer. V. a. pour ${ }^{(119)}$ faire mal au coeur, faire soulever le coeur pour estomac. La moindre chose l'écoure, pour lui donne le mal de cœur. Il est facile à écoeurer. ce ragout m'a écœuré. Cette femme est si mal propre qu'elle écoure. Ecœuré. ée. part. On l'emploie aussi comme sub. C'est un écœuré, un dégouté.

x Ecœurant, te. adj. ce couteau est écœurant, pour malpropre. \&cc. vous avez des mains écoeurantes \&c. On dit aussi d'un homme extrêmement sale $\&$ malpropre, quel écourant morceau!

On l'emploie aussi comme sub. quel est cet écourant? ce malpropre -

Ençà. Interjection - pour Çà. Ex. Ença, travaillons. Ençà, part [sic] vite. Ençà, depêche-toi. $x$ Ecolter $\}$ V. a. Avoir l'estomac découvert d'une Escolter $\}$ manière indécente. Qui vous a écoltée? 
elle est toute escoltée. Il ne se dit guère que des femmes.

x Ebrâiller. V. a. Il a même signification qu'écolter elle est ébraillée, il est revenu tout ébraillé. il se dit d'un homme qui vient de se battre et dont l'estomac est tout à découvert; voyez comme le voilà ébraillé. On dit aussi substantivement, d'une fille publique c'est une ébraillée, et aussi d'une fille trop découverte. (se débrailler est francois - $)^{(121)}$

\section{[CAHIER B]}

Ecossois. sub. mas. Nom de Nation dont on se sert dans cette phrase, ${ }^{(122)}$ galeux comme [un] Ecossois.

x Exemple - par exemple façon de parler adverbiale usitée ainsi : oh par exemple, c'est trop mentir, au lieu de - oh pour le coup \&c.

$x$ Ebrayer. v. a. Il se dit du bétail à corne[s] quand il frappe de ses cornes. La vache a ébrayé ou corné ${ }^{(123)}$ l'enfant. prends garde que le bouf ne t'ébraye. Il se dit aussi d'un homme qui a été battu bien rudement, un tel l'a ébrayé.

$x$ Etriver. V. a. pour agacer, taurmenter [sic]. $\mathrm{Ne}$ m'étrive ${ }^{(124)}$ pas tant. Il l'a fait étriver.

$x$ Etrivant. te. sub. Il est étrivant. c'est ${ }^{(125)}$ une étrivante.

Equilibre. sub. mas. on dit en francois cela est dans l'équilibre, mettre dans l'équi[li]bre, $\&$ figurément, mettre, tenir dans l'équi libre, pour mettre, tenir dans l'égalité. Ici nos habitans font souvent usage de cette phrase : Je suis dans lin $\approx$ quilibre [sic], si je ferai ceci ou non, \& tout simplement, je suis dans l'inquilibre; pour ${ }^{(126)}$ exprimer qu'ils sont indécis, irrésolus, sur le choix, ou qu'ils ${ }^{(127)}$ doutent du sort de telle affaire. \&c. 
Epicailles. sub. fém. mot employé dans cette phrase, et qui n'est pas françois. Il lui en a donné sur les épicailles, il t'en don $\approx$ nera sur les épicailles, pour dire, qu'il l'a bien ${ }^{(128)}$ grondé, qu'il te grondera bien.

$x$ Ecrapoutir.

Ecrapoutiller ${ }^{(129)}$ par le poids de quelque chose, ou par quelque effort.) Je t'écrapoutirai le nez d'un coup de poing. Si je vais à toi je t'écrapoutis. il a mis le pied sur cette araignée, (130) cette grenouille et les a écrapoutillé [sic] On dit aussi d'une personne qui en a écrasé ${ }^{(131)}$ une autre de ses coups, ${ }^{(132)}$ il l'a écrapoutillé [sic], ou ${ }^{(133)}$ écrapoutillé [sic] comme un crapaud. On y joint le pronom personnel il s'est écrapoutillé la main avec le marteau, elle s'est écrapoutillée contre le mur dans la place. ${ }^{(134)}$ Ecrapoutillé, ée. part. nez, ${ }^{(135)}$ écrapoutillé, pour aplati, court; doigt écra $[\approx]$ poutillé, écrasé par un coup.

s'Ejârer. V. - on l'emploie, avec ${ }^{(136)}$ écartiller pour écartiller \& écarquiller, dans le vrai sens de ces derniers vers, $c$-à $d$. écarter, ouvrir les jambes. il s'est éjâré il a tombé tout éjâré.

s'Ebarouir. V. - Il se dit des ouvrages de tonnellerie qui s'ouvrent, sechés par(137) le soleil ou la chaleur du feu, ou ${ }^{(138)}$ autrement. La cave est humide, mettez-y les seaux, ils ne s'ébarouiront pas là. Vous avez laissé sécher la cuve, la voilà ébarouie; mettez-la vite dans l'eau.

Empocheter. V. a. (mettre en poche.) II ${ }^{(139)}$ a empocheté aujourd'hui 15 minots d'avoine.

Equiper V. a. \& se faire équiper. V. pour maltraiter, battre. Il l'a mal ${ }^{(140)}$ équipé; il s'est fait équiper comme 
il faut. J'ai été mal équipé à ce combat, pour estropié. ${ }^{(141)}$

Equipage. sub. m. On dit dans le même sens que l'on emploie équiper : dans quel équipage ${ }^{(142)}$ reviens-tu ! qui t'a mis dans cet équipage? pour, où as-tu été maltraité, qui t'a battu de la sorte?

Epotraillé (adj:)(143) la poitrine décou[ $[\approx]$ verte. Il est épotraillé. ${ }^{(144)}$

Eborgner - V. act - (rendre borgne) Il l'a éborgné. - il a failli l'éborgner. ${ }^{(145)}$

Epinette. - pour Arbre de vie - ou - du paradis. ${ }^{(146)}$

\section{$\underline{\text { F. }}$}

[CAHIER A]

Flasque. (Flaque) mot bas $\&$ populaire. ${ }^{(147)}$ $x$ Flasque, Sub. mas. (un paresseux - Dictionnaire de Boyer). on l'emploie ici pour peureux - On dit d'un homme qui a manqué de courage dans quelque occasion, c'est un flasque. Il est aussi employé dans sa vraie signification, ou plutôt, pour expri mer un homme qui n'a pas de cœur au travail. - (mot populaire.)

x Flasque, adj. (qui est sans force, sans vigueur - Dictionnaire Boyer) Cette acception n'est pas usitée ici. On dit d'une étoffe qui n'a point ou qui a perdu son apprêt, qu'elle est flasque, ${ }^{(148)}$ cette soie est flasque, cette indienne est flasque, $\mathrm{c}-\mathrm{à}-\mathrm{d}^{(149)}$ est mollasse.

x Flasquer. V. n. Avoir peur. Ex : il a flasqué dans telle occasion. (populaire)

$x$ Flasquer. V. qui n'est employé qu'à

l'infinitif. \& dans cette seule phrase : 
fers à flasquer, pour fers à repas ser le linge.

Faire coup. manière de parler qui nous vient de nos pères et qui signifie aller combattre. Quand partironsnous pour faire coup?

Factionnaire, sub. mas. Nous faisons de ce mot un substantif, quoiqu'il ne soit qu'un adjectif. Ainsi au lieu de dire, un soldat factionnaire - obli gé à faire faction - on dit simplement un factionnaire : on a trouvé le factĩ onnaire mort dans sa guérite. ${ }^{(150)}$

$x$ Fesser. V. act. (Fouetter, frapper sur les fesses avec des verges ou la main) Ceci ne se dit que d'un enfant : fesser un enfant. Cependant on dit tous les jours, sans attention, d'un homme qui a été fouetté (sur le dos) par la main du bourreau; qu'il a été fessé. Un tel est condamné par la Cour à être fessé tel jour, sur la place du marché. C'est improprement parler. Fréte, ${ }^{(151)}$ sub. mas. pour froid. Il géle de fréte. pour froid. Le fréte est bien grand. II fait fréte. Je l'écris de cette manière pour exprimer qu'on prononce le ${ }^{(152)} \mathrm{T}$ final.

Fréte, Fréde. adj. pour froid, froide.

Ex. tems fréte, froid; fréte comme glace; mains frédes, froides.

x Fenouil. sub. fém. pour Fenouil sub. mas. (plante aromatique.) On dit : Il y a de la fenouil dans ce tabac, pour du fenouil.

$x$ Fenouillette. sub. fém. (espèce de pomme qui

a le gout du fenouil.) Cette acception n'est pas reçue ici, mais on dit fenouil lette pour fenouil. Ce tabac sent la fenouillette.

Fard. sub. mas. employé pour Farce sub. fém. (mélange de diverses viandes, ou 
seulement d'herbes, d'œufs et d'ingrédiens, hachés menu et assaisonnés, qu'on met dans le corps de quelques animaux, ou dans quelque autre viande.) ce fard est excellent, pour cette farce est excel $\approx$ lente. Fard de haut goût, pour Farce. Bien peu de personnes employent ici le vrai mot.

Foin. sub. mas. On dit ici : faner pour fener le foin. Une Veuilloche, un mulon de foin. Voyez Veuilloche - Mulon.

x Fàro. sub. mas. petit maitre, et plus particu $\approx$ lièrement, petit maitre de campagne. C'est un faro; il est beau comme un faro de campagne ; il fait le fâro.

$x$ Fàroder. V. a. faire le faro, il farode rudement. faire l'amour, il farode Mademoiselle $\mathrm{N}$.

$x$ Fine-boutique. sub. fém. terme de dérision pour ${ }^{(153)}$ signifier un pauvre esprit, un homme dont l'esprit est borné. C'est une fine boutique; tu peux bien en parler, tu es une fine-boutique pour cela!(154)

Fièrement. adv. On l'emploie quelquefois, pour beaucoup, bien - fièrement riche, fière $[\approx]$ ment bon, fièrement sot; il a fière $[\approx]$ ment du grain; il en a fièrement.

Flâner. V. n. pour paraisser, faire le pares $\approx$ seux. Il a flâné toute la journée. Il n'est bon qu'à flâner.

Flandrin. sub. mas. ce mot est françois, (c'est un ${ }^{(155)}$ sobriquet que l'on donne aux hommes élancés et qui n'ont pas une contenance ferme, c'est un grand flandrin.) On l'ems ploie ici pour paresseux; tu ne seras jamais qu'un flandrin; c'est le plus grand flandrin que \&c.

Fricasser V. a. On emploie ici ce verbe, pour faire cuire, - dissiper (il a fricassé tout son bien en moins de rien) - on dit aussi, cet argent est fricassé, ${ }^{(156)}$ c'est autant de fricassé, et toutes ces acceptions sont françoises. Mais on lui 
donne d'autres significations. Ex. il lui a fricassé une bonne ramasse, une bonne dégelée, phrase populaire qui signifie qu'il l'a battu rudement. on dit encore je me fricasse de tes menaces, je m'en fricasse, pour je m'en moque. ${ }^{(157)}$

\section{[CAHIER B]}

$x$ Fine-boutique. sub. fém. On emploie ce mot pour exprimer aussi, un matois, un rusé. $\mathrm{Ne}$ vous y jouez pas, c'est une fine bou $\approx$ tique, ou un fin matois, que l'on emploie aussi. (158)

x Fignoler. V. n. On dit d'un jeune garçon qu'il fignole, qu'il commence à fignoler, quand il se met à faire des dépenses d'éclat, soit en habits, voitures \&c. et faire le galant. Eh! comment, il voudroit fignoler.

$\mathbf{x}$ Fignoleur. sub. mas. un homme qui fignole, c'est un fignoleur, il devient fignoleur.

Finir. V. a. - on l'emploie avec la préposition avec pour signifier - n'avoir plus besoin, ou $^{(159)}$ achever, terminer \& simplement finir. J'ai fini avec cela, je n'en ai plus besoin; j'ai fini avec votre livre, je l'ai lu, j’ai achevé de le lire ; j'ai fini avec cet ouvrage, j'ai achevé, fini cet ouvrage. Ils ont fini avec leur dispute, ils ont terminé, leur dispute. expression Angloise. ${ }^{(160)}$

Fectif, ive. adj. certain, sûr, vrai. c'est une chose fective; Monsieur est ici, je l'ai vue [sic], c'est fectif. Il est un fectif honnête homme \&cc.

Flambe. sub. fém: - (Vieux mot qui signifioit autrefois la flamme du feu) On l'emploie encore ici dans ce sens.

$x$ Flambant, flambante - adj. Comme participe de Flamber (jetter des flammes), ce mot est françois, mais comme adjectif il ne l'est pas. - On le joint le plus communément à l'adj. neuf : il avoit hier un habit tout flambant neuf, un cha 
peau tout flamblant ${ }^{(161)}$ neuf. Ces expressions sont sans doute figurées \& veulent exprimer ${ }^{(162)}$ le lustre de l'habit et du chapeau; de même que ces phrases - un couteau tout flambant neuf, une épée toute flambante neuve, font allusion au poli de ces instrumens. On dit dans ce même sens figuré, en bon françois - des épées flamboyantes. tout flambant nud, se dit aussi d'une personne entièrement nue. ${ }^{(163)}$ On dit encore : il lui a passé son épée toute flambante au travers du corps, pour toute entière, ou j'usqu'à la garde.

Fripper. V. actif. Il n'est pas françois dans le sens de fripponner, voler, tromper quelqu'un, comme on le dit quelque ${ }^{(164)}$ fois. Ex : Il a frippé son hôte d'un mois de pension, ou, il l'a frippé de la bonne manière, de telle somme \&c.

Ferlasser. V. n. Il se dit du bruit que fait une étoffe roide que l'on manie. Voilà une indienne qui ferlasse beaucoup.

Ferlassement. s. m. de Ferlasser. c'est bien incommode d'entendre toujours ce ferlassement. Ma robe fait un tel ferlassement, quand je marche, que j'en suis dégouté (165).

Fanals - sub. mas. plur. de fanal usité communément au lieu de fanaux. Il y a beaucoup de fanals dans les rues.

\section{G.}

[CAHIER A]

x Gâ.- sub. m. Ce mot est employé par tous les habitans de la campagne pour signifier un jeune garçon. On ne se sert guère de ce mot sans $y$ ajouter 
l'adjectif petit. Où est le petit gâa?

Je vous enverrai mon petit gâ.

Vous donnerai $[s i c]$ ceci au petit gâ.

Viens ici, mon gâ.. ${ }^{(166)}$

$x$ Guide - sub. fém. longue rêne attachée

à la bride d'un cheval attelé. Voyez

cordeau \& courroie. -

x Gavache. sub. mas. Ce mot qui dans

sa vraie acception signifie coquin

misérable, est employé ici comme

synonime de poltron. On le fait

même féminin et l'on dit : c'est une

vraie gavache.

Garde-corps. sub. mas. Mot que l'on employe ici indifféremment avec celui de Garde-fou qui est le mot propre, pour exprimer les balustres que l'on met au bord des ponts, des quais \&c.

Gouette, sub. fém. Sa gouette grossit tous les jours, pour son goêtre $8 \mathrm{c}$.

Gérémium. sub. mas. pour Géranium, Géra niome, Bec-de-grue. - plante.

Guedou - baiser en guedou, pour se faire retaper, style badin. Voyez Baiser.

$x$ Gueusasse $^{(167)}$, sub. fém. que nous employons

pour gueusaille, s. f. c-a-d. Canaille.

Nous nous servons aussi de gueusaille.

Cé [sic] n'est que de la gueusasse; il ne loge chez lui que de la gueusasse.

Galipote. sub. fém. Pour bordel, boucan.

Ce jeune homme court la galipote.

Graissoux, se. adj. il n'est pas françois. ${ }^{(168)}$

il signifie gras, grasse (sali, imbu de graisse ou de quelque matière onctu $\approx$ euse.). où as-tu mis ton chapeau, qu'il est si graissoux; ôtez ce linge graissoux, joues, mains graissouses. Il se dit aussi substantivement d'une personne mal-propre, c'est un graissoux, une graissouse. 
x Gaspiller. V. a. (dissiper par toutes sortes de dépenses inutiles le bien dont on a la disposition : - il a gaspillé son bien en peu de tems.) On dit aussi à peu près dans le même sens, gaspiller des hardes, du linge, du fruit, [(] $\underline{\text { style }}$ familier.) On dit ici gaspiller son butin pour toutes ces choses. - $\underline{B u t i n}^{(169)}$

$x$ Gouliâ. sub. mas. Ce substantif répond à ce qu'on appelle en françois - gouliafre, goulu, tous deux adj. (Il se dit d'une personne qui mange avidement \& malproprement) il mange comme un gouliâ, c'est un gouliâ. On dit en francois dans ce sens, c'est un vrai gouliafre, c'est un homme extrêmement goulu.

Graffigner. V. a. (Entamer et déchirer légèrement la peau avec les ongles.) il lui a tout graffigné le visage, il l'a graffigné inhumainement. - Je crois qu'il y a cette différence entre graffigner et égratigner, que l'égratignure peut se faire avec toute autre chose que les ongles, une épingle par exemple, au lieu que par égrafignure nous n'enten dons que la blessure faite avec les ongles.

$x$ Georges. sub. fém. pour orges. s. f. employee [sic] dans cette phrase proverbiale faire ses georges, faire bien ses georges, pour orges, - pour dire faire son profit, faire bien ses affaires. Style familier.

Glumer. V. a. On dit d'un joueur qui a beaucoup perdu au jeu qu'il s'est fait glumer, qu'on lui a glumé tout, ou simplement qu'on l'a glumé; dans ce dernier sens il signifie perdre beaucoup. On dit d'un fils ou d'une maitresse 
prodigues, qu'ils ont glumé, qu'ils glument le bon-homme, pour dire qu'ils font beaucoup de dépenses.

Grichou. s. m. Ce substantif signifie tantôt malin et tantôt laid; c'est un petit griz chou, il est malin comme un grichou. Dieu! quel grichou! n'est-elle pas laide comme un grichou.

Griche-poil. s. m. sinonime de grichou comme malin - espiègle. Voyez ce grichepoil!

Gricher. V. a \& n. pour grincer les ou des dens.

[CAHIER B]

$x$ Ginseng. s. mas. - plante. La chute de cette branche de commerce en Canada, a donné naissance à cette manière proverbiale ${ }^{(170)}$ de parler : tombé comme le ginseng, $c-\grave{a}-d$. tout-à-coup \& sans espérance de se relever - On dit d'un homme qui n'est plus en faveur, qui est tombé dans le discrédit, qui n'a plus de popularité, et qui a éprouvé ces inconstances inopinément, $\underline{\text { il est tombé }}$ comme le ginseng.

Graisse - Graisser - Voyez - Méz

cher \& moucher - (sinonime) - (171)

Game - Game-coq. sub. mas. (prononcez guiéme) mot absolument Anglois.

$x$ Giboulée de neige : Voyez Bordée - abât. ${ }^{(172)}$

\section{H.}

[CAHIER A]

x Habitant - Sub. mas. Ce [sic] le nom que

l'on donne à notre agriculteur, ou laboureur. Il y avoit bien des habitants au marché d'hier.

x Habitante, sub. fém. La femme de l'Habitant. 
x Houiller. V. a. Verbe trivial usité parmi les habitans pour changer de che vaux. Un habitant à cheval ou en voiture, qui en rencontre un autre à cheval ou en voiture, lui crie : houille, houille ${ }^{(173)}$; changeons, chan $\approx$ geons. Veux-tu houiller? Houiller. V. a. s'emploie dans ${ }^{(174)}$ ces phrases : je suis houillé de ma femme, j'en suis las, dégouté. Je suis houillé de mon hôte, il me déplait, me pèse sur les épaules. Je suis houillé de la vie, las de vivre. Se Houiller. V. r. Il se houilla de vin, il se gorgea de vin.

x Hausse, sub. fém. Sorte de ${ }^{(1 / 5)}$ vêtement en usage chez nos sauvages, qui sert à couvrir la jambe. On l'appelle aussi Mitasse. voilà de jolies hausses ou mitasses.

Herbe à dinde.

Herbe à la puce.

\section{J.K.}

[CAHIER A]

Incendie. sub. mas. Beaucoup de personnes disent : il y a eu une grande incen $\approx$ die. c'est une faute. On doit dire, un grand incendie.

$x$ Improuver. V. n. Cette terre improuve tous les jours, pour dire qu'elle s'amé $\approx$ liore; ce jeune homme improuve à vue d'œil, pour exprimer, qu'il fait de rapides progrès, qu'il $\underline{\text { se }}$ perfectionne bien sensiblement.

$x$ Jument. sub. fém. nom que les voyageurs dans le Nord Ouest donnent à une caisse de fusil[s]. 
x Juifrèsse. sub. fém. pour Luive. c'est

une juifrèsse, pour une femme Luive.

Icit. - pour Ici. adv. de tems \& de lieu.

Impropre, adj. des 2 genres (qui ne se dit que

du langage, mot, expression impropres, ${ }^{(176)}$

$\mathrm{c}-\mathrm{a}-\mathrm{d}$. qui ne convient pas, qui n'est

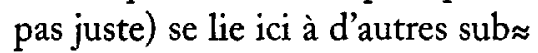

stantifs, ${ }^{(177)}$ Improprement.

x Immatériel, elle. adj. (qui est sans mélange ${ }^{(178)}$

de matière, ${ }^{(179)}$ - les substances, les

formes immatérielles. terme didactique)

Cette faute est bien immatérielle, pour

légère \&c.

$x$ Inconsistant, te. adj. Il n'est pas françois -

pour incompatible, contraire, contradictoire.

On dit aussi - c'est bien inconsistant de

votre part de... pour inconsidéré. Anglois -

$x$ Jouquer. V. n. pour Jucher. V. n. On ne

l'emploie guères qu'avec le pronom per $\approx$

sonnel. Les poules se jouquent, où

cet homme s'est-il allé jouquer,

pour se juchent, jucher.

x Jouquoir. sub. mas. pour juchoir. s. m.

$x$ Joli-cœur - Ce mot n'est employé qu'avec

le verbe s'appeller dans ce sens :

à vous voir disposer de ces fruits, je

vois que je m'appellerai joli-cour,

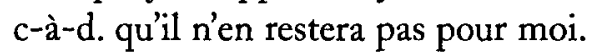

Tout le monde se servit, et je m'appellai

joli-coeur, c-à-d. je n'eus rien.

\section{$\underline{\mathbf{L}}$}

\section{[CAHIER A]}

$x$ Licher. - V. a. pour lécher. licher un plat, s'en licher les barbes; (passer la langue sur quelque chose.) 
On dit familièrement d'un fils qui est dépensier ${ }^{(180)}$, il en a bien liché, il en lichera bien à son père, il lui en liche!

$x$ Lichefrite, sub. fém. pour Lèchefrite. $s$. f. Lévier. sub. mas. pour Evier, (conduit par où s'écoulent les eaux, les lavures et les autres immondices d'une cuisine \&c.) On dit : le trou d'un lévier, au lieu d'un évier. Un lévier de pierre, pour un évier de pierre. Jetter des ordures par le lévier, au lieu de, jetter des or $\approx$ dures par l'évier.

$x$ Légerte, pour légère, féminin de léger. Ex :

la compagnie légerte. c'est une fille bien légerte. Il a la tête extrêmement légerte.

$x$ Loucheur-se -(181)

\section{M.}

[CAHIER A]

Moindrement. adv. pour Le moins du monde. ${ }^{(182)}$

Matelot. - sub. mas. Nom que l'on donne à un joli petit insecte.

x Mouiller. V. n. employé presque exclua sivement ici à (183) celui de pleuvoir. Il mouille, il a mouillé, il mouil lera demain; au lieu de il pleut, il a plu(184), il pleuvra. \&c.

Le verbe ${ }^{(185)}$ mouiller en bon françois n'est jamais employé que comme v. a. tremper, humecter, rendre humide. Ex. la pluie a mouillé les chemins; mouiller un linge dans l'eau; mouiller l'ancre, ou sim $\approx$ plement mouiller, pour dire jeter l'ancre pour arrêter le vaisseau. On l'emploie aussi dans ce cens, ici. 
x Marier. V. a. assez souvent employé pour Epouser, prendre en mariage. Ex. Monsieur O. a marié Mademoiselle P. pour a épousé ou s'est marié à ... Monsieur marie une telle \&c. Il se dit toujours de l'Epoux. Ce mot est de la bonne compagnie. - Anglois.

$x$ Mondaine, adj. pour Mondée. Il n'est employé par quelques personnes que dans cette phrase : orge mondaine, pour orge mondée. -

x Micoine. sub. fém. vase de bois qui sert de cuiller aux Sauvages.

Malin. gne. adj.- c'est malin. Voyez Rustique. c'est malin de plaider avec son Seigneur. ${ }^{(186)}$

Méchant. te. adj. - c'est méchant. Voyez Rustique.

Micmac. sub. mas. Ce mot qui signi[fi]e en bon françois - Intrigue, manigance, pratique secrète $^{(187)}$ dans quelque mauvaise vue, Ex : il y eut bien du micmac dans cette af $\approx$ faire - n'est point employé ici dans cette acception; mais au lieu de baragouin, baragouinage, mots dont on se sert aussi, dans leur vrai sens. Je n'entends rien à ce micmac; il parle micmac; bon Dieu ! quel micmac! c'est du micmac pour moi. On dit aussi d'un baragouineur, c'est un micmac.

Marinage. sub. mas. pour Marinade sub. fém. chose marinée. (bouillie dans le vinaigre) Je n'ai pas de marinages, cette année. maria nage de ${ }^{(188)}$ cornichons, d'oignons.

Mulon. s. m. mot dont on se sert ici au lieu de Meule, dans cette phrase, un mulon de foin. Mulon est toujours une grosse meule. Voyez Veuilloche.

Manche. sub. mas. (partie d'un instru $\approx$ ment par où on le tient.) On emploie encore ce mot au lieu de ${ }^{(189)}$ queue. Ex. le manche de la poêle, d'un poë lon. \&c. le manche d'une pipe. ${ }^{(190)}$ 
$\left.\begin{array}{l}\text { Menoirs. sub. fém. Pl } u r . \\ \text { Travail. sub. mas. Sing. }\end{array}\right\}$ Voyez Travail.

$x$ Mèche. sub. fém. Pour exprimer la grande distance qu'il y a d'un endroit à un autre, on dit : $\mathrm{Oh}$ ! il y [a] une mèche; il a une mèche à faire.

$x$ Mèche. sub. fém. Nom que l'on donne à l'extrémité la plus déliée d'un fouet, et qui est d'ordinaire une petite ficelle rapportée - Il a usé, il a perdu la mèche de son fouet.

$x$ Mècher, V. a. Il équivaut à Rosser.

$\mathrm{Si}$ je vais à toi, je te mècherai d'importance. Il mèchoit ${ }^{(191)}$ son cheval sans pitié.

Moucher. V. a. Il a la même significaz tion que mècher, avec cette diffé $\approx$ rence pourtant qu'on l'emploie plutôt contre un enfant que contre un homme fait, ou un animal. Son père l'a bien mouché; fanfan, je te moucherai, si tu ne te tais.

x Mitasse. sub. fém. Voyez Hausse. Mitasses neuves.

x Mâle. sub. mas. Vêtement de tête. C'est ${ }^{(192)}$ le nom que donnent nos habitans à un bonnet de laine, de couleur, qu'ils portent journellement. Ils l'appellent plus communément Tuque. Où est mon mâle? As-tu vu ma tuque?

Matelat. sub. mas. Terme de chasseur. Nom que l'on donne ici à un [sic] espèce de trait qui se décoche avec un arc. Il est différent de la flèche, en ce qu'il y a une grosse tête à une de ses extrémités. Ex. l'arc, le matelat et les flèches. Vous avez l'arc et les flèches, il ne vous manque plus $\mathrm{qu}^{\mathrm{r}^{\prime(193)}}$ un matelat. 
[CAHIER B]

Manchonier [sic], sub. mas. (artisan qui travaille en pelleterie) il n'est pas françois, c'est Fourreur, marchand pelletier. Envoyez mes mitaines chez le manchonnier, chez le fourreur.

Moindrement. adv. Il a plusieurs significations. Il n'est pas le ou la moin $\approx$ d[r]ement fatigué, orgueilleux \&c. pour pas du tout. - Le moindrement que vous lui en parlerez, il se fâchera, pour pour peu que vous lui en parliez. \&c. quand il vente la moindrement, pour un peu. -

Major, re - adj. pour Majeur, re adj. dans ces phrases. la force majore, affaire majore, intérêt major, la majore partie et au jeu de carte tierce majore.

x Mistimus - en mistimus. c'est écrit en mistimus, il a fait cela en mistimus. Matériel, le ${ }^{(194)}$. adj. On dit c'est une affaire bien matérielle ${ }^{(195)}$, pour, de grande conséquence; question matérielle, pour importante. C'est un point matériel de savoir... pour c'est un point essentiel \&c. Anglois.

Mal. sub. mas. On se sert de l'expression, tomber d'un mal, pour signifier l'Epilepsie, que l'on nomme aussi mal caduc, haut mal, mal de Saint Lean, mal de Saint. Il tombe du mal-caduc, du haut mal.

Manquer - V. - (pour faillir) il a manqué périr. il l'a manqué belle. il a manqué mourir. ${ }^{(196)}$

Maskinongé - sub. mas. (poisson). Espèce de Brochet.

Montréaliste. - de Montréal.

Mauvaisete ${ }^{(197)}$. sub. Synonime de méchanceté.

Ex : Il est d'une mauvaiseté à toute épreuve.

Il vous fait là des mauvaisetés, sans doute. 


\section{N.}

[CAHIER A]

Notureau, sub. mas. C'est le nom que l'on donne à un jeune cochon, que l'on engraisse pour tuer. Lorsqu'il est tué il prend le nom de porchais. Ex : Vous ${ }^{(198)}$ avez-là de beaux notureaux; ce notureau est bon à tuer, il fera un beau porchais. Voyez Porchais.

Niveleux, se. adj. Il se dit, en fait d'ou $\approx$ vrages, de ceux qui requièrent beau coup d'application d'esprit, de tems, et ${ }^{(199)}$

de dextérité. Ex. peindre en miniature, démêler de la soie, faire un calcul ${ }^{(200)}$ difficile, éta blir le compte particulier de chaque héritier d'une succession où(201) il y a des enfans de plusieurs lits \&c. sont des ouvrages niveleux.

$x$ Nijon, sub. mas. - employé pour bout d'homme

Voyez Bougon.

Nix, ou Nix for stein, mots pris de l'Allemand employés de cette manière; il croyoit la trouver à la maison, ma[i]s Nix, elle étoit déjà partie, pour mais point. On dit aussi, Nix-nix, tu ne l'auras pas \&c.

$x$ Naturel, elle, adj. pour salubre, adj. qui contribue à la santé. C'est un air naturel, une nourriture naturelle. cette tisanne est bien naturelle, prenez-en. \&c.

Niveleux. on applique cette epithéte a des ouvrages, difficiles par leur tènuite [sic], qui exigent beaucoup de dextérité, de temps et de patience, par exemple on dira ce doit être un ouvrage niveleux que de faire, des Eguilles, des Epingles : parce qu'on s'imagine, que c'est difficile, et long.(20) 


\section{O.}

[CAHIER A]

Office. Sub. mas. \& fém. Ce mot qui ne doit s'employer que dans ces phrases, rendre de bons offices, c'est l'office d'un bon père de..., assister à l'office divin, $j$ 'ai tel office dans l'administration, le Saint Office (l'inqui[ $\approx]$ sition[)], et qui dans tous ces cas est masculin; ou comme voulant désigner le lieu ou ${ }^{(203)}$ l'on garde la vaisselle, le linge et ce qui concerne la table, ou les cuisines et qui alors est féminin, - a cependant ici une acception plus étendue. Ainsi les archives d'un Notaire sont mon office; un avocat vous envoye à son office trouver ses clercs. Un homme en place vous prie de passer à son office, qu'il vous y donnera audience à telle heure. Tout homme public, tout homme d'affaire [sic], le marchand même a ${ }^{(204)}$ son office : c'est le coin de sa ${ }^{(205)}$ maison où chacun de ces Messieurs barbouille du papier. Les mots étude, bureau, cabinet, sont ${ }^{(206)}$ trop vieux \& trop communs! - Anglois - (201)

$\mathbf{x}$ Orignal, pour, Elan, - animal sauvage.

Oreilles. sub. fém: - de souliers; mot usité pour les tirans des souliers.

Ouète ${ }^{(208)}$. s. f. pour ouate, s. f. (espèce de coton, plus fin que le coton ordinaire.) Le Dictionnaire de l'académie admet ces deux manières d'écrire $\&$ de prononcer ce mot. Il dit aussi qu'on écrit $\&$ prononce ${ }^{(209)}$ communément, de la ouate, de la ouète, comme on le dit ici.

Mais il écrit : une camisole d'ouate, 
une jupe doublée d'ouate, une couver $\approx$ ture d'ouate; tandis que nous ne faisons pas l'élision et que nous disons de ouète. Nous ne [nous] servons ici que du mot ouète \& nous faisons toujours l'hiatus, quand le mot est précédé d'une voyelle. -

Obligé, ée. part. On dit souvent : je vous serois obligé pour ce couteau, au lieu de, si vous me donniez \&c. je vous serai obligé pour ce livre \&c. Anglois.

On emploie de la même manière remercier. ${ }^{(210)}$

$x$ Originer. V. n. provenir, tirer son origine; son Etymologie, dériver. Ex. d'où faitesvous originer ce mot, pour dériver? Je ne sais d'où cet homme origine, pour, d'ou [sic] il tire son origine, ou quelle est son origine. Cette maladie origine d'un amas d'humeur; d'où croyez-vous qu' origine cet abus? pour provenir.

x Ordonné, ée, part. Je suis ordonné par

Monsieur S. de vous dire... pour j'ai ordre de Monsieur S. de... Elle est ordonnée d'aller à Québec, elle a ordre, ou reçu ordre \&c.

Otocas. - sub. - (plante.)

Oiseau blanc - pour Ortolan.

\section{$\underline{\mathbf{P}}$}

[CAHIER A]

x Paré - part. du verbe parer v. act. On se sert par toute la Campagne et assez géné ralement dans la bonne Compagnie de ce participe pour l'adjectif prêt, prête. Etes-vous paré à partir?

Madame est parée à monter en voiture.

x Payer. v. act. Plusieurs personnes \& surtout du bon ton vous disent : j'irai demain, Monsieur vous payer une visite; je viens de chez Madame ... lui payer mes respects mes complimens. 
L'action de ${ }^{(212)}$ rendre ne leur est pas famiz lière : Je me rappelle à ce sujet d'une phrase d'un ${ }^{(213)}$ homme qui a eu l'honneur malheureusement de siéger trop longtems à notre Chambre d'Assemblée, et dont d'aveugles léche-crachats s'enrumoient [sic] à préconiser les prétendues lumières, dire un jour de son siége : "On me verra toujours paré à payer le regard dû à l'objet en question.» Anglois.

Poudrerie. sub. fém: On dit qu'il a fait une grandre $[s i c]$ poudrerie, une poudre rie affreuse, quand en hyver la neige soulevée par un gros vent -

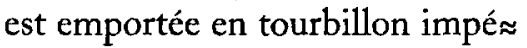
tueux. C'est, si l'on peut le dire, une bourasque de neige.

Poudrer. v. n. - Ce verbe n'est usité que dans cette phrase : il poudre, (214) il a poudré bien fort; pour exprimer qu'il y a, ou qu'il y a eu une grande poudrerie.

Pesâ. sub. mas. sing. C'est le nom que les habitans donnent à la tige des pois, lorsqu'ils en ont ôté le grain en le battant. Le pesâ sert alors de nourriture aux animaux.

Plairie, sub. fém. quelquefois usité pour Prairie. $x$ Pipe, sub. fém. sorte de mesure de distance chez quelques-uns de nos habitans. Combien $y-a-t-i l$ d'ici à tel endroit? - environ 5 pipes, c'. a.d. 15 lieues, la pipe comptant pour environ 3 lieues. On dit encore : il y a d'ici là une pipe! une belle pipe ! pour dire que la distance est grande. Voyez Mèche.

$x$ Poudine $\}$ sub. fém. Mêts anglois du \& Poutine $\}$ nom de pouding. Il se dit 
aussi en françois mais il est masculin \& se prononce poudingue quoiqu'il s'écrive pouding. Ex. Un pouding au $\underline{\text { riz }}$; il y a des ${ }^{(215)}$ poudings ${ }^{(216)}$ de plusieurs sortes.

Porchais. sub. mas. Petit cochon mort, ${ }^{(217)}$ de quatre mois ou environ, que l'on a engraissé pour tuer. Il se nomme notureau, j'usqu'au moment qu'on le tue.

Porceline sub. fém. - pour Porcelaine.

Pourceline $\int$ tasse, assiette de pourceline ou porceline, pour, de porcelaine.

Pelote. sub. mas. [sic] au lieu de Paume. s. f. (sorte de jeu où jouent deux ou plusieurs per $\approx$ sonnes qui se renvoient une balle avec une raquette ou avec un battoir, dans un lieu préparé exprès.) On emploie ici le mot de pelote, non seulement pour expriz mer le lieu où on joue, un jeu de pelote, mais encore pour la balle même, on a perdu la pelote, ${ }^{(219)}$ et pour le jeu même, viens jouer à la pelote. on doit dire paume, dans tous ces cas. Pelote est françois dans ces expressions : pelote de fil, de soie, de neige, ${ }^{(20)}$ de laine, ou pelotons.

Palette. sub. fém. on s'en sert au lieu de battoir il est françois. Voyez Pelote.

$x$ Peter. v. a. pour claquer. ce fouet péte bien, faire peter son fouet. (habit, mains, vitres -) pour crever - son fusil lui a ${ }^{(221)}$ peté dans la main. Je crains qu'il en péte.

Papier-nouvelle, sub. mas. pour papier-public. (222) Pistolage $\}$ sub. fém. [sic] sorte de limaçon du genre Pucelage $\int$ de la porcelaine. Pucelage est admis en françois.

$x$ Prouvable. adj. 2 genres (qui peut être prouvé[)]. Ce fait est prouvable, au lieu de dire qu'on peut prouver ce fait.

Port-épic \}ub. mas. nom que l'on donne ici à un Porc-épic animal qui, comme le Porc-épic, porte des piquans au lieu de poil, mais 
qui est plus ${ }^{(22)}$ petit $^{(24)}$ que le vrai porcépic. - C'est une sorte d'Hérisson, ou le coendou, ou urson.

$x$ Plaisant. te. adj. employé mal-à-propos, pour beau ou agréable, dans ces phrases : un tems est plaisant, une plaisante après dinée. - Anglois.

[CAHIER B]

Pincettes. sub. fém. plur. Baiser en pincettes, pour se faire retaper, Voyez Baiser.

Plated. adj. anglois dont on se sert commu $\approx$ nément au lieu de fouré [sic], ée participe. pour ${ }^{(225)}$ les vases et autres meubles de cuivre dorés ou argentés d'un côté. J'ai acheté deux chandeliers plated, pour fourrés. Il a un beau pot plated, fourré.

Pelleter. V. a. remuer, jetter avec une pelle. pelleter la neige, ou de la neige; pelleter du blé, pour mettre du blé dans un sac avec la pelle. Il est aussi neutre il a pelleté, tout le jour.

Pesant. sub. mas. pour Cauchemar s. m. qui se prononce comme cochemar. (sorte d'oppression ou d'étouffement qui survient quelquefois durant le sommeil, en sorte qu'on croit avoir un poids sur l'estomac, et qui cesse dès qu'on vient à se réveiller.) il est sujet au pesant, avoir le pesant, pour le cauchemar; il a eu le pesant toute la nuit.

Pochetée, sub. fém. pour Poche (226) le contenu d'une poche[D]. Il lui a été volé, cette nuit, près de 3 pochetées de bled. On dit aussi poche dans ce sens.

Passe-rose. sub. fém. - pour -(227) rose-d'outremer, ou rose-tremière; c'est la Mauve des jardins.

Poisson doré. -

Poisson blanc. -

Perdrix - pour Faisan sauvage. 


\section{Q.}

[CAHIER A]

x Quêteur, euse. sub. qui ne se dit en françois que d'une personne qui quête pour quelqu'un, est non seule $\approx$ ment employé ici dans ce sens, mais plus généralement au lieu de Mendiant, sub. mas. \& de Quémandeur, euse, sub.

$x$ Quêter. v. pour mendier, quémander.

$x$ Quitte - [(]pour acquet) vous avez plus de quitte d'aller là -(28)

Quitter. V. (pour laisser) je l'ai quitté chez lui, à la maison. il l'a quitté mourir. (229)

Québecquois ou Québéquois. - Gens de Québec.

\section{$\underline{\mathbf{R}}$.}

[CAHIER A]

x Relevée. sub: fém. (tems de ${ }^{(230)}$ l'après dinée).

Ce terme est employé ici dans sa vraie signification, mais comme il ne doit être d'usage que dans le Bareau et qu'on ne s'en sert guères qu'à Québec, il seroit bon de le restituer, de peur de chicane. à deux heures de rele $\approx$ vée, pour de l'après-dinée.

$\mathrm{x}$ Ramancher. V. act. et canadien. Il a plusieurs significations.

$\left(1^{\circ}\right.$ - conter avec diffusion $\left.{ }^{(231)}\right)$ Que me ramanchez-vous?

Il me ramancha une histoire qui n'avoit ni queue ni tête. ${ }^{(232)}$

( $2^{\circ}$ raccommoder réparer $\left.{ }^{(233)}\right)$ Il a bien ramanché ce chandelier. La caléche est bien ramanchée.

Robe de bœuf. sub. fém. (terme de commerce)

On appelle ainsi la peau du buffle 
préparée d'un seul côté. Elles servent l'hyver de lit et de couvertures de lit aux gens de la Campagne, et dans les carioles à couvrir ou envelopper les jambes des Voyageurs.

$x$ Retraiter. v. n. ${ }^{(234)}$ terme de

guerre pour exprimer qu'une armée se retire, qu'elle fait sa retraite. Ex : L'armée a retraité de deux lieux [sic]. Le Général se vit contraint de retraiter, pour dire qu'il a été obligé de faire retraite, de se retirer. - Anglois.

x Retraiter v. act. terme de pratique; pour (235)

Retrayer $\int$ retraire, $c-a ̀-d$ retirer par droit de parenté, ou par droit seig $\approx$ neurial, un héritage qui a été vendu. Ces deux verbes ne ${ }^{(236)}$ sont employés par les notaires qu'à l'infinitif \& au partiz cipe. Ex : avec le droit de retraiter ou retrayer, pour retraire. J'ai retraité, ou retrayé cette terre, pour j'ai retrait \&c.

Remou ${ }^{(23))}$. sub. mas. C'est le mouvement rétrograde que font les eaux, après un courant rapide. (238) Racérer. V. a. (mettre de l'acier avec du fer, afin de rendre celui-ci propre à couper.) on doit dire acérer. acérer une hache, et non racérer.

Racéré, éé. [sic] adj. pour acéré, éé [sic]. Une lame, Une pointe, une hache acérées.

Rôle - sub. mas. mot pris de l'anglois pour exprimer ces petits pains que l'on vent $[$ sic $]$ dans les rues le matin \& le soir, pour le thé. - c'est proprement la miche (pain d'une grosseur médiocre, pesant au moins une livre, \& quelque [sic] fois deux[)]. Voulez-vous des miches? Le crieur de miches est à la porte. 
x Résous. - participe du verbe Résoudre. Il est bien souvent employé et mal-à-propos pour Résolu. (ce participe n'est d'usage qu'en parlant des choses qui se changent, qui se convertissent en d'autres; et il ne se dit point au féminin. Brouillard résous en pluie.) - Ex : Je suis résous à plaider. Je l'ai résous à venir avec moi, à se battre; pour je suis résolu à plaider; je l'ai résolu à venir \&c. Rustique. adj. 2 genres. Cet homme est rus $\approx$ tique, pour dire qu'il est impossible de gagner quelque chose sur lui : la colline est rustique à monter, pour difficile, c'est un cas rustique, difficile à débrouiller. les chemins sont rustiques, c.à.d. mauvais. On dit familièrement : c'est rustique, pour exprimer, difficile, épineux, fatiguant [sic]. On emploie dan[s] ces mêmes sens malin \& méchant.

Retontir. V. n. pour Rebondir. v. n. (faire des bonds.) On dit d'une pelote que l'on jette contre le plancher - qu'elle retontit bien, pour rebondit. - On l'emploie aussi pour éprouver des secousses, le coup de canon a été si fort, ou le canon a peté si fort, que toute la maison en a retonti. Le bois a retonti de ses cris, pour expria mer l'effet de l'écho. Les vitres ont retonti du coup de terre [sic], pour ont été ebranlées, ont tinté. (retentir.)

Remercier. V. a. Voyez obligé.

Ramasse. sub. fém. Synonime ici de volée de coups. Il lui a donné une bonne ramasse. Voyez dégelée, \& fricasser.

Ratapiat. sub. mas. pour baragouin, ou langage qu'on n'entend point. quel ratapiat!

$x$ Reintier. s. m. mot employé par les habitans pour les reins, quelquefois ${ }^{(239)} \mathrm{de}$ l'homme, $\&$ toujours $^{(240)}$ des animaux. Du reintier d'un cochon on 
peut faire trois socs. il est foible du reintier.

à (Re)brousse-poil ${ }^{(241)}$. façon de parler adv. à contre-poil.

On dit ici figurément; ne parlez pas à Monsieur il est à rebrousse-poil aujourd'hui, c-à-d. de mauvaise humeur.

[CAHIER B]

Rhimb - pour Rhumb de vent. sub. mas.

Cette ligne suit ou court ${ }^{(242)}$ tel $\underline{\mathrm{Rhimb}}$ de vent.

$x$ Rapiester. V. a. pour rapiécer (remettre des piéces à un habit ou à du linge -).

Je suis à ${ }^{(243)}$ rapiester ses chemises.

On emploie aussi ${ }^{(24)}$ ce verbe absolu $\approx$ ment : on n'est occupé qu'à le

rapiester, pour dire - rapiécer le linge de telle personne. Aussi rapiester quelqu'un.

x Rapiesté - tée - part. habit rapiesté, veste rapiestée. cet homme est tout rapiesté.

Regricher - Les cheveux lui regrichoient sur la tête. (245)

à Regriche ou griche-poil -(246)

\section{$\underline{\mathbf{S}}$}

[CAHIER A]

Sentinelle. sub. fém. (soldat qui fait le guet.) On dit ici assez généralement :

Le sentinelle doit avoir froid par ce tems; on devroit dire, la sentinelle \&c. Voyez Factionnaire.

Style. - sub. mas. Nos faiseurs de mots ont donné à ce mot en françois toute la latitude ${ }^{(24)}$ qu'il a dans la langue Angloise. Ainsi l'on dit : la table de Monsieur ${ }^{(248)}$ étoit servie dans le plus haut stile; sa maison est meublée dans le grand stile; madame est habillée 
dans le grand stile, pour exprimer que rien n'est ${ }^{(249)}$ épargné, que tout est de meilleur gout et de la der $\approx$ nière mode. Ce sont les rabats de la bonne faiseuse du Marquis de Molière. ${ }^{(250)}$ Anglois.

x Sieau. Sub. mas. pour seau; un seau ferré, des seaux de bois.

Sorcière. sub. fém. ${ }^{(251)}$ tourbillon de neige ou de poussière, ${ }^{(252)}$ occasionné subite ment par un fort ${ }^{(253)}$ vent, et de peu de durée. Fermez les fenêtres, de crainte que la sorcière ne les brise. Avez-vous vu passer la sorcière? Etiez-vous dehors durant la sorcière, pour : quand elle passoit ${ }^{(234)}$.

$x$ Soulier. sub. mas. On donne ici le nom de souliers françois, aux souliers de cuir ${ }^{(255)}$ qui viennent de l'étranger, ou que les cor $\approx$ donniers manufacturent; celui de sou $\approx$ liers de boeuf, à ceux que les habitans se font de la peau du bœuf; et celui de souliers sauvages, à ceux que font ces derniers de la peau du chevreuil.

$x$ Suspect. te. adj. (qui est soupçonné, ou qui mérite de l'être). Nos habitans em $\approx$ ployent aussi ce mot pour susceptible. dans ce cens : Elle est suspecte, $c^{\prime}-\grave{a}-d$. Elle est facile à blesser.

Stellci - Stellcit. pron. démonstratif celui-ci. pron. dém. Stellà, pour celui-là. pron. dém.

Souris-chaude, sub. fém. pour Chauve-souris. oiseau.

$\mathbf{x}$ Sur. préposition de lieu. - Employée très souvent pour $\underline{\text { Chez, }}$, préposition. Je viens de sur mon oncle; je vais sur le notaire. Je dine sur ma tante.

$x$ Sarpidon. sub. mas. pour tapageur; c'est un petit sarpidon, quel sarpidon.

Soigner. V. a. (traiter avec beaucoup de soin, il a été bien soigné durant sa maladie.) on dit aussi soigner des enfans, 
Sur. ure. adj. que l'on emploie communé ment pour aigre; du vin sur, du lait sur, pour aigres. aigre signifie ici vice; mais pour marquer la qualité, on dit sur, des pommes sures, des fruits surs, l'oseille ronde est fort sure.

$\mathbf{x}$ Surir. V. a. pour aigrir (rendre aigre, faire devenir aigre.). La chaleur ${ }^{(257)}$ surit, ou fait surir le lait. \& $\mathrm{x}$.

$x$ Sauvagesse, sub. fém. pour Sauvage. -

voilà une sauvagesse, pour une sauvage.

Sucet. sub. mas. On appelle ainsi le tuyau du blé d'Inde, ou sa tige, dégarnie de ses épis, et que l'on fait manger au bétail à corne[s]. Les sucets, coupés un peu verts, font de bons fourages. Quand les sucets sont trop secs, les animaux ne les manche $[s i c]$ point. ${ }^{(258)}$ tu as là de beaux sucets. On l'appelle aussi coton de bled d'Inde, quoiqu'on donne plus particulièrement ce nom à l'épi dégarni de ses grains.

Soupe. - Il dort comme une Soupe.

Sorel. habitant de Sorel. Ex. C'est un $\underline{\text { Sorel. }}$ Voilà des sorels assurément, leur habillement le dit.

\section{T.}

[CAHIER A]

$\mathbf{x}$ Tuer - V. act. Ce verbe est quelques fois employé pour éteindre, assez improprement. Tuez la chan $\approx$ delle; avez-vous tué le feu du poele ${ }^{(25)}$, au lieu de éteignez, avez-vous éteint ${ }^{(260)}$. \&ce.

Traîne. sub. fém. Sorte de voiture d'hyver, qui sert aux habitans pour charrier le bois, les denrées, le foin, \&c. Voyager On l'appelle en françois ${ }^{(261)}$ traineau. 
Traîne de clisse. sub. fém. Voiture d'hyver des Sauvages, qui leur sert à trans $\approx$ porter leur venaison $\&$ autres choses. C'est une planche très mince, courbée par devant.

Traineau. sub. mas. C'est la traine des Canadiens, mais d'une dimension beaucoup plus petite, assez souvent avec des bras. Elle sert aux enfans à courir et glisser sur la neige.

Trompe. Sub. fém. petit instrument de $x$ Bombarde. S. fém. fer, qui a une lan guette au milieu dont on tire un son en le mettant entre les dents et en le touchant avec le bout du doigt. On l'appelle ordinaire ment trompe à laquais, ou Guim $\approx$ barde. ${ }^{(262)}$ Nos habitans le nomment tantôt Trompe et tantôt Bombarde.

Travail. sub. mas. Ce mot qui ne s'emploie qu'au singulier, se dit des deux piéces de bois qui se prolongent en avant d'une charette, ou que l'on attache par une chaîne de fer aux ${ }^{(263)}$ voitures d'hyver en Canada et entre lesquels [sic] est placé le cheval qui les traine; c'est proprement le brancard. Le mot travail est employé à Montréal \& celui de menoirs au pluriel à Québec. Le travail de la traîne est cassé; les menoirs de la cariole ${ }^{(264)}$ sont neuves.

Thétière, sub. fém. pour Théière, vase pour faire infuser le thé.

Tondre. sub. mas.

$x$ Tuque. sub. fém. vêtement de tête. Voyez Mâle. Tauraille. sub. fém. Nom sous lequel les habitans désignent en général les jeunes veaux et ${ }^{(265)}$ genisses (taure). Ce sont de belles taurailles, où sont les taurailles. une jeune tauraille. 
Tire. sub. fém. Sorte de sucre en bâton que l'on procure ${ }^{(267)}$ de la melasse, en la faisant bouillir. De bonne tire; de la tire pleine d'yeux; la tire se vend un sol le bâton.

Tapisserie. sub. fém. ${ }^{(268)}$

Tirans. sub. mas. plur. pour aurore boréale.

Il y a beaucoup de tirans dans le

Nord. - Voyez Clairon -

$x$ Tapon. sub. mas. Ce mot s'emploie ici pour Tas, Paquet. touffe ${ }^{(269)}$. Un Tapon de laine, tapon de neige, la neige tombe en tapon, pour dire qu'elle tombe par flocons. - Un tapon de graisse. On dit d'un petit enfant gros et gras, c'est un tapon, c'est un tapon de graisse.

$x$ Train. Sub. mas. On appelle train les occupations quotidiennes du ménage. Mettez vous à votre train; vous n'avez pas encore commencé votre train, vous êtes bien paresseuse. Vous ne finirez pas votre train aujourd'hui. (270)

On dit être en train, pour ivre. Il commençoit à se mettre en train, Il n'étoit pas mal en train, il est revenu en train. Ils sont tous en train.

Trèfe. sub. mas. pour Trèfle (herbe, et une des quatre couleurs d'un jeu de cartes[)].

Troisse. - pour Trois. nombre cardinal.

$x$ Tricoler. v. $n$. Il se dit d'un ${ }^{(271)}$ homme ivre, qui chancelle. Il tricole, je l'ai vu dans la rue tricolant de la belle sorte.

$x$ Tapin. sub. mas. synonime de tape ou soufflet. il n'est pas françois.

x Trempe adj. 2 genres au lieu de trempé, ée, part. Il est ${ }^{(272)}$ tout trempe, Son habit est tout trempe, pour dire qu'il a été extreme[ [ ] ment ${ }^{(273)}$ mouillé; il est tout trempe de sueur, pour dire qu'il a beaucoup sué.

On dit proverbialement, il est revenu trempe comme une ${ }^{(274)}$ soupe, pour trempé, pour dire très mouillé. 
$\mathbf{x}$ Touche. sub. fém. terme en usage parmi les fumeurs, dans ces phrases. Veux-tu fumer une touche, viens fumer une touche, après avoir fumé une touche, pour dire fumer un peu et tout au plus une pipe.

Tête. sub. mas. [sic] On dit d'une personne entêtée, opiniatre, c'est une tête d'escargot, une tête de boule.

[CAHIER B]

$x$ Tinton. sub. mas. Il n'est pas françois. On dit le tinton sonne, on sonne le tinton, pour exprimer (le son lent d'une cloche dont le battant ne touche que d'un côté.) on doit simplement dire : on tinte à la Paroisse, on tinte la messe, le sermon, ou absolument, la cloche tinte; on peut aussi dire tinter la grosse, la petite cloche.

Tourniquet - sub. mas. - tournoiement d'eau dans un trou fait dans la glace d'une rivière, particulièrement le printems. Voyez Remous. - (27)

\section{V.}

[CAHIER A]

x Voyage. sub. mas. J'ai payé 2 sols pour un voyage d'eau, au lieu de dire pour une voie d'eau. On appelle voie d'eau $\&$ non pas voyage, les deux seaux d'eau que porte un homme. On dit aussi Voie, pour charretée. Ex : voie de bois, voie de pierre, voie de sable.

$x$ Vulgaire. adj. 2 genre[s]. Outre son vrai sens, nos habitans entendent par vulgaire visible, évident. c'est vulgaire qu'il est midi. Il est vulgaire que tu perdras ton procès.

x Vire-l'œil. sub. mas. pour louche d'un œil. 
Il est employé par dérision. Veuxtu te cacher, vire-l'oeil!

Veuilloche. sub. fém. On nomme ici une petite meule de foin. Mettre le foin en veuilloches, c'est le mettre en petits tas dans les prairies, avant de le charrier. ${ }^{(276)}$ On appelle Mulon une grosse meule de foin.

x Verbalement. adv. Il a plusieurs accep tions. Il lui a parlé verbalement pour sec. On dit qu'un postillon mène ses chevaux verbalement, pour bien vite, bon train. il l'a battu verbalement pour bien fort.

Vaches (plancher des) c'-à-d. la terre. Ex :

Une fois sur le plancher des vaches, tout ira bien, dira quelqu'un voyageant sur l'eau.

\section{Notes du manuscrit 1}

1. sont surcharge est raturé.

2. une acception semblable ou contraire; surcharge la même acception raturé.

3. Orthographié ainsi dans le ms.

4. est surcharge étoit raturé.

5. le ajouté.

6. Amanchure non défini ajouté au verso de la première page, en regard de l'article Amancher.

7. Accent aigu raturé sur le e.

8. Orthographié ainsi dans le ms.

9. arrête surcharge ne passe raturé.

10. et non raturé.

11. te. ajouté.

12. adj. surcharge sub. mas. raturé.

13. Orthographié ainsi dans le ms.

14. avons raturé.

15. ânât écrit ainsi dans le $\mathrm{ms}$.

16. employé dans le ms.

17. sub. mas. Espèce de bourlet de vieux linges, ou guenilles que les habitans mettent sur le cou du bouf qui labourre, pour le lui garantir du mal que surcharge sub. mas. Ce mot est quelque fois usité dans la Campagne, pour chapeau de 
femme : Un apichimon de paille, le reste illisible se termine par mon apichimon, le tout raturé.

18. dans surcharge pour aise raturé.

19. Article au crayon.

20. Article au crayon raturé.

21. attisèe dans le ms.

22. e surcbarge a; bombe raturé.

23. habitans ajouté dans l'interligne au-dessus de les pauvres.

24. Escarpolette, ajouté.

25. Les enfans surcharge Ta sœur ber[lancille] raturé.

26. s final raturé.

27. dont surcharge que raturé.

28. (civière) ajouté au-dessus de brancard.

29. L'accent grave remplace l'accent aigu dans les deux occurrences de calèche d'abord orthographié caléche.

30. Sous-entendre : voir l'article Gaspiller dans lequel figure le mot butin.

31. que l'on surcharge qui et début de mot illisible, le tout raturé.

32. s final raturé.

33. e final raturé.

34. lit raturé.

35. nomme surcharge dit raturé.

36. d' raturé.

37. (V.L.B.) [= Voyez Livre B] écrit verticalement dans la marge.

38. $2^{\circ}$ le rivage opposé raturé.

39. enclin à surcharge porté facile[ment] raturé.

40. Accent grave supprimé.

41. elle remplace il.

42. maltraitrer dans le ms.

43. ou autrement écrit au crayon surcharge et de légers coups raturé.

44. le prélude de battre raturé.

45. C'est - Brusquer écrit verticalement au crayon dans la marge.

46. $\mathrm{r}$ final surcharge $\mathrm{x}$.

47. pour bourasque écrit verticalement au crayon dans la marge.

48. \& remuez ajouté.

49. rétapé dans le $m s$.

50. s'est fait baiser remplace a été baisé.

51. en guedou ajouté.

52. er écrit au crayon surcharge re de la première syllabe.

53. Accent supprimé sur e.

54. er écrit au crayon surcharge re de la première syllabe.

55. a bredassé remplace n'a fait que bredasser. 
56. maltraiter surcharge gronder raturé.

57. de coups raturé.

58. à surcharge pour raturé.

59. le raturé.

60. ma remplace la raturé.

61. Orthographié ainsi dans le ms.

62. (V.L.A) [= Voyez Livre A] écrit verticalement dans la marge.

63. Voyez plus loin renvoie pour la suite à la page suivante dans le ms.; c'est le même article Beurrer qui se continue à la page suivante; Beurrer V. a. n'est pas une autre entrée, mais un rappel dans le haut de la nouvelle page dans le $\mathrm{ms}$.

64. r surcharge $\mathrm{z}$.

65. c'est Bavarder écrit verticalement au crayon dans la marge.

66. bat dans le ms.

67. le plus surcharge pour raturé.

68. Orthographié ainsi dans le ms.

69. c'est surcharge veut raturé.

70. ma surcharge la raturé.

71. donne surcharge prete raturé.

72. à surcharge un début de mot illisible raturé.

73. nomme surcharge donne aussi raturé.

74. qu'il est beaucoup surcharge que le chem[in] raturé.

75. de neige. surcharge pour en jetter la neige dans le suivi d'un mot illisible, le tout raturé.

76. Article raturé.

77. ne et ici ajoutés.

78. couper surcharge arracher de terre raturé.

79. signifie le surcharge ne peut s'appliquer qu'au raturé.

80. les surcharge aux raturé.

81. (V. Livre B.) écrit verticalement dans la marge.

82. Orthographié ainsi dans le ms.

83. Ebrayer ajouté.

84. prononcez Cris ajouté au crayon.

85. Article raturé.

86. Cage \& Cajeux raturé.

87. On dit nomme cajeux raturé.

88. on dit : courir les côtes pour les campagnes ajouté.

89. (V. Livre A.) écrit verticalement dans la marge.

90. Virgule supprimée.

91. de cabaret ou taverne. surcharge auberge raturé.

92. à l'armée. surcharge dans une can[tine] raturé.

93. cabaretier ou tavernier surcharge aubergiste raturé. 
94. Même remarque qu'à la note précédente.

95. s'amuser à raturé.

96. se promener surcharge faire aller raturé.

97. Virgule supprimée, suivie de en ajouté au crayon puis raturé.

98. la charge d'un canot, surcharge le canot \& son chargement, raturé à l'exception de le qui a été oublié, et que nous n'avons pas restitué.

99. bled d'inde, ou il lui a donné un beau bled d'inde; surcharge l'article Clairon raturé et recopié plus bas.

100. Article au crayon.

101. nos surcharge on l'a raturé.

102. de bateau ajouté.

103. Cette occurrence et la précédente sont orthographiées ainsi dans le ms.

104. En raturé.

105. de surcharge pour raturé.

106. On l'emploie avec le pron. pers. Elle est raturé.

107. sur raturé.

108. act. \& n. surcharge act. raturé.

109. commun ajouté.

110. je raturé.

111. de ajouté.

112. L'accent grave remplace l'accent aigu,

113. éé dans l'entrée et dans avariéés.

114. la surcharge une raturé.

115. dans cette chambre, ajouté.

116. là raturé et ici un instant, surchargent un peu, je suis raturé.

117. L'accent aigu et l'accent grave se croisent au-dessus du e dans le ms.

118. peu surcharge moins raturé.

119. dégouter raturé.

120. On dit d'une personne raturé.

121. (se débrailler est francois-) écrit verticalement dans la marge.

122. fier \&c. raturé surcharge fier comme un Ecossois raturé.

123. ou corné ajouté au crayon.

124. m'étrivez surcharge m'agacez raturé.

125. c'est surcharge Il est aussi adj. raturé.

126. dire raturé.

127. ont des raturé.

128. bien surcharge beaucoup raturé.

129. Ecrapoutiller ajouté au crayon.

130. ce supprimé par nous.

131. écrasé surcharge battu raturé.

132. de ses coups, surcharge d'une manière horrible raturé. 
133. ou surcharge \& raturé.

134. dans la place. ajouté.

135. doigt raturé.

136. avec surcharge au lie[u] raturé.

137. qui s'ouvrent, sechés par surcharge lorsqu'ils s'ouvrent par la raturé.

138. simplement raturé.

139. se dit raturé.

140. mal surcharge bien raturé.

141. pour estropié. ajouté.

142. équivage dans le $\mathrm{ms}$.

143. Epotraillé remplace Epotrailler; (adj:) ajouté.

144. Article au crayon.

145. Article au crayon.

146. Article au crayon.

147. Article raturé.

148. pour signifier qu'elle raturé.

149. c-à-d surcharge pour raturé.

150. Article raturé.

151. adj. raturé.

152. te dans le $m s$.

153. exprimer raturé.

154. (V.L.B.) [= Voyez Livre B] écrit verticalement dans la marge.

155. c'est un surcharge il se dit des raturé.

156. e final raturé.

157. À partir de et toutes ces acceptions jusqu'à moque., le texte se trouve au bas de la première colonne de la lettre $G$ qui est en regard.

158. (V.L.A.) [= Voyez Livre A] écrit verticalement dans la marge.

159. avoir fini de raturé.

160. expression Angloise au crayon écrit verticalement dans la marge.

161. tout ajouté; flamblant ortbographié ainsi dans le ms.

162. veulent exprimer remplace expriment.

163. tout flambant nud, se dit aussi d'une personne entièrement nue. ajouté au bas de l'article; signe de renvoi dans le texte.

164. Orthographié ainsi dans le ms.

165. e final raturé.

166. Voyez Gars écrit verticalement au crayon dans la marge.

167. Gueusasse remplace gueusaille.

168. il n'est pas françois. surcharge plein, trempé de graisse raturé.

169. Article raturé. Le renvoi à Butin était inutile puisque gaspiller ne figure pas dans les exemples donnés dans l'article Butin.

170. proververbiale dans le $\mathrm{ms}$. 
171. Article au crayon.

172. Article au crayon.

173. s final raturé dans les deux occurrences.

174. dans surcharge pour raturé.

175. de surcharge d'habill[ement] raturé.

176. Parenthèse fermante supprimée.

177. et a d'autres raturé.

178. L'accent aigu remplace l'accent circonflexe dans mélange d'abord écrit mêlange.

179. Parenthèse fermante supprimée.

180. est dépensier surcharge a beaucoup dépensé d'argent raturé.

181. Entrée au crayon.

182. Article raturé.

183. la raturé.

184. Accent circonflexe supprimé.

185. franco[is] raturé.

186. c'est malin de plaider avec son Seigneur. ajouté dans la marge; signe de renvoi dans le texte.

187. L'accent grave remplace l'accent aigu.

188. cocombres raturé.

189. au lieu de surcharge pour exprimer la raturé.

190. On dit (nomme au-dessus raturé) aussi le manche d'une (de la au-dessus raturé) pipe, la partie la plus déliée de la pipe : toute cette phrase est raturée.

191. L'accent grave remplace l'accent aigu.

192. un b[onnet] raturé.

193. ne et plus qu' ajoutés.

194. el raturé.

195. s final raturé.

196. Article au crayon.

197. il n'y a pas d'accent aigu sur le e final de mauvaiseté dans l'entrée.

198. que l'on engraisse pour tuer. Lorsqu'il est tué il prend le nom de porchais. Ex: Vous surcharge Vous avez là de beaux notureaux. Il est en usage parmi les habitans. On se sert aussi du mot raturé.

199. d'adresse raturé.

200. compte raturé; un peu raturé.

201. le raturé.

202. Article ajouté sur un bout de papier collé au bas de la page.

203. ou sans accent ajouté.

204. Accent grave supprimé.

205. coin de sa surcharge lieu où cha[cun] raturé.

206. cabinet, sont surcharge est raturé.

207. Boutique de Notaire écrit verticalement au crayon dans la marge. 
208. L'accent grave remplace l'accent aigu.

209. Soulignement supprimé sous on écrit \& prononce.

210. remercier. surcharge un mot illisible raturé.

211. mes complimens. ajouté.

212. L'action de surcharge Le mot raturé.

213. une dans le ms.

214. Virgule ajoutée; bien fort raturé.

215. il y a des surcharge les raturé.

216. sont raturé.

217. mort, ajouté.

218. avant raturé.

219. mais raturé.

220. \& pelote ou peloton ou raturé.

221. a surcharge est raturé.

222. Article raturé.

223. plus surcharge trop raturé.

224. pour raturé.

225. pour surcbarge des cha[ndeliers] raturé.

226. plu[tôt] raturé.

227. Grande Mauve, ou raturé.

228. Article au crayon.

229. Article au crayon.

230. tems de surcharge après diner raturé.

231. avec diffusion ajouté.

232. Que me ramanchez-vous? raturé.

233. réparer ajouté.

234. pour exprimer raturé.

235. dire raturé.

236. ne ajouté.

237. s final rature.

238. C'est le mouvement rétrograde que font les eaux, après un courant rapide. surcharge au crayon Tournoiement d'eau dans une rivière raturé et suivi de Voy Tourniquet au crayon raturé.

239. quelquefois surcharge tant raturé.

240. \& toujours surcharge que raturé.

241. Préfixe Re raturé.

242. ou court ajouté.

243. le raturé.

244. emploie aussi surcharge se sert de raturé.

245. Article au crayon.

246. Article au crayon. 
247. l'étendue ajouté au crayon dans l'interligne au-dessous de latitude.

248. est raturé.

249. rien n'est remplace tout est sans raturé.

250. Cet homme peint dans le grand stile, pour de la [mot illisible] vouloir exprimer qu'il peint d'une manière supérieure, mais bien de la manière la plus à la mode. raturé.

251. léger raturé.

252. ou de vent ajouté au crayon et raturé.

253. un fort au crayon surcharge le raturé.

254. passoit remplace a passé.

255. de cuir ajouté.

256. Article raturé.

257. La chaleur surcharge Le tonnerre raturé.

258. point. surcharge plus raturé.

259. Accent grave supprimé sur le premier e de poele.

260. éteignez, et éteint surchargent tuez et tué raturés.

261. On l'appelle en françois surcharge deux mots illisibles suivis de on appelle, le tout raturé.

262. ou Guimbarde. surcharge Bombarde est le nom raturé.

263. aux surcharge aux traîneaux, traînes et autres raturé.

264. s final raturé.

265. et surcharge ou.

266. On s'en raturé.

267. procure surcharge retire raturé.

268. Entrée raturée.

269. touffe surcharge \&c.

270. On appelle train les occupations quotidiennes du ménage. Mettez vous à votre train ; vous n'avez pas encore commencé votre train, vous êtes bien paresseuse. Vous ne finirez pas votre train aujourd'hui. surcharge Ce mot s'emploie ici pour dépenses de luxe, d'éclat, telles que voitures $8 x$. Cet homme mene un gros train. Il fait beaucoup de train. raturé.

271. e final raturé.

272. Il est surcharge Je suis raturé.

273. extremement surcharge mouillé raturé.

274. une surcharge la raturé.

275. Voy. - Remous. - raturé. Article au crayon.

276. Ce so[nt] raturé. 


\section{[MANUSCRIT 2]}

Amancher pour Emmancher, V. actif (Mettre un manche.)

$\mathrm{Si}$ ce verbe n'étoit jamais employé que dans son sens propre, celui d'emmancher, - et que l'on ne commit d'autre faute que de le prononcer mal, je ne m'arrêterois peut-être pas à en faire ici mention; mais les signiz fications qu'on lui donne, et qu'il n'a pas, autorisent sans doute l'annotation que j'en fais. -

Il sert à exprimer Raccommoder. Ex : Ce Ciseau, cette porte sont bien amanchés ou ramanchés. Voyez ce verbe. Il a amanché cela, on ne peut pas mieux. Il signifie encore Refaire. Ex : Oh bien! s'il l'a défait, il peut bien l'amancher ou le ramancher. Allumer. v. actif.

Quand on ne joint point de régime à ce verbe, il est toujours entendu que c'est de la pipe dont on veut parler. Ex : - Entrons allumer. Veux-tu allumer? Quand tu auras allumé, tu me donneras du feu. Il signifie aussi se reposer, comme dans ces phrases : Quand je serai au bout de ce guérêt, j’allumerai. Mes enfants, quand vous aurez $z^{(1)}$ achevé cette partie de votre ouvrage, vous pourrez allumer; je vous le per $\approx$ mêts [sic]. Ce dernier cens du verbe vient de [ce] que nos fumeurs mettent toujours à profit leur loisir \& leur repos. Allumer s'emploie aussi pour visiter, mais alors il est toujours régi par quelque verbe. Ex : Un habia tant dira à un de ses amis : si tu passes dans notre Paroisse, arrête allumer chez moi, ou entre, viens allu mer à la maison; c'est-à-dire, viens me voir.

Ahurissant. - te. Adjectif verbal.

Cet Adjectif, formé sans doute du participe présent d'Ahurir, (verbe François qui veut dire interdire, 
étonner, rendre stupéfait,) n'est pas lui-même François, et est d'ailleurs employé ici comme synonime d'ennuy ant. Ex : - Cette personne est bien ahurissante. On ne peut se figurer combien il est ahurissant. Dieu! que ce séjour est ahurissant!

Il est quelquefois substantif, - Finis donc, ahurissant;

Apichimon. sub. mas. $1^{\circ}$ Bourrelet de

linge ou de paille, que l'on met sur le cou du bouf qui labourre, pour empêcher que le joug ne le blesse. $2^{\circ}$ Morceau d'étoffe, peau de mouton, ou autre chose semblable, que nos habitans mettent en guise de Selle, lorsqu'ils montent à cheval.

$3^{\circ}$ Méchant lit, grabat. Ex : Faites-lui un apichi mon. Le moindre apichimon fera mon affaire.

Ce mot vient du Sauvage.

Arse. sub.(2)

qui n'est nullement françois. On l'emploie dans ces phrases : - Cette chambre est si petite, qu'on n'a pas l'arse d'y mettre un lit; - pour qu'il n'y a pas moyen \&c. J'aime à avoir de l'arse, partout où je suis ; - pour être à mon aise, ou point gêné. Il n'y a pas dans ce lit l'arse de se retourner, ou assez d'arse pour y coucher deux; - pour exprimer qu'il est bien étroit, $o u^{(3)}$ qu'il n'est pas assez large.

On voit par ces exemples que ce mot est le synonime Canadien de place, espace.

Abât. sub. mas.

Il est fort commun d'entendre dire : nous avons eu, depuis quelque tems, de grands abâts de pluie, - pour de grandes pluies, ou de fréquens orages. Nous aurons sous peu quelque abât de nége, ou quelque Bordée. Voyez ce mot. - Il n'est pas François.

Appointement. sub. mas. (pension, gages ${ }^{(4)}$ des Officiers d'un Grand.) -

Appointer. - Verbe actif. (Donner des appoin temens.) Ce Verbe, françois dans cette acception, est employé ici pour - Nommer à une charge ou place. Ainsi l'on dit : Il a plu à Monsieur le Gouverneur d'ap $\approx$ pointer Monsieur C. D. Grand-Voyer du District, au 
lieu de nommer Monsieur C. D. Grand-Voyer. \&c. On apprend que le Chevalier P. est appointé Gouverneur de telle Province, pour nommé Gouverneur.

Bombe. sub. fém. ou Canard. sub. mas. pour bouilloire, sub. fém. C'est le vase dans lequel on fait bouillir l'eau pour le thé. Le dernier de ces mots n'est pas du tout en usage, qu'oiqu'il [sic] soit celui dont on devroit se servir. On dit donc: Mettez la bombe au feu. Apporz tez le canard. Remplissez d'eau la bombe, le canard; au lieu de la bouilloire.

La bombe se dit plus communément dans le district de Québec, et le canard dans celui de Montréal.

Boucane. sub. fém. au lieu de Fumée. Ex :

Quelle boucane il y a ici! Exposer quelque chose à la boucane. La boucane étoit si forte, si épaisse, qu'on l'auroit coupé [sic] avec un couteau. manière de parler très commune.

Quoique les François aient le verbe Boucaner, ils ne se servent pas du mot boucane.

Boucaner. V. a. (Faire secher du poisson et de la chair ${ }^{(5)}$ au soleil ou à la cheminée, comme font les Sauvages \&c.) Il est françois dans ce sens; ainsi on peut dire, boucaner des peaux. De la viande boucanée. Des jambons boucanés. Mais quelque fois [sic] on emploie ce verbe comme neutre, et l'on dit : cette maison boucane trop, pour l'habiter. votre poële boucane beaucoup. Cette cheminée a le défaut de boucaner. Toutes ces phrases ne se peuvent dire; il faut employer Fumer.

Berlancille. sub. fém. pour Balançoire,

Escarpolette ou Brandilloire. Jeu d'enfant. (Corde, branche d'arbre ou planche avec quoi on se balance, on se brandille. .) On ne peut donc plus dire : viens voir ma berlancille.

Berlanciller. V. n. pour Se Balancer ou se Brandiller, Verbes Réfléchis. -

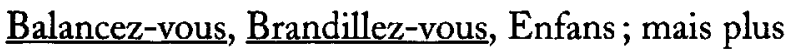
de berlancille.

Butin. sub. mas.

Mot François dont on fait ici un emploi abusif. Les effets, les meubles, les hardes d'une personne sont chez nous 


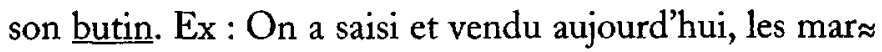
chandises, les meubles, les hardes, les livres, enfin tout le butin d'un tel. Votre butin (vos effets) restera en ma possession, j'usqu'à [sic] ce que vous m'ayez payé. Il s'est échappé de sa pension et a emporté tout son butin; c'-à-d. tout ce qui lui appartenoit. C'est mon butin, pour mes hardes \&c. Il gaspille tout son butin, $c^{\prime}-\mathrm{à}-\mathrm{d}$. son linge, ses habits. Butin est un de ces mots revenus des Camps avec nos Ancêtres, et qui joint à beaucoup d'autres de ce genre, épars dans cet ouvrage, seroient seuls, aux yeux d'un penseur, une preuve presque suffisante du génie militaire \& de la vie guerrière des Canadiens d'alors; si l'histoire ne nous avoit transmis leurs actions. Tout le monde sait que butin ne se dit que de ${ }^{(6)}$ ce qu'on prend sur les ennemis.

Bordée. sub. fém. Terme marin que nous employons dans cette phrase : nous avons essuyé une furieuse bordée de nége. On dit aussi Abât dans le même sens. Voyez ce mot. On dit aussi absolument : il a tombé hier une grande bordée. Car la bordée s'applique à la nége seule; tandis que l'abât se dit de la nége et de la pluie. On ne dit donc pas une bordée de pluie, mais un abât de nége se dit tous les jours.

Bicler. V. n. au lieu de Loucher. v. n. Ex : Il bicle; elle a le malheur de bicler. En substituant loucher à ce verbe, on parleroit correctement.

Bicleux. se. adj. pour Louche. adj. des 2 genres. Il est bicleux. Sa sœur est bicleuse, pour louche. On l'emploie aussi comme substantif. Ex : C'est un bicleux, - une bicleuse. - Louche n'est jamais substantif. Cet adjectif a pour synonime Loucheur, se. adj. et virel'œil, sub. ${ }^{(7)}$ Voyez ces mots. Il est sans doute inutile de remarquer que ${ }^{(8)}$ ces mots sont impropres, et que louche est le seul bon.

Brâsser. V. actif. (Remuer avec les bras, à force de bras. Ex. Brasser de l'or, de l'argent fondu.) On donne ici une autre acception à ce verbe, et l'on dit : je l'ai brassé d'importance, - dans le même sens que l'on dit en françois, - je l'ai savonné (reprimandé(9).) A l'aide d'une petite figure de Rhétorique, et 
de ce verbe, les Canadiens font un bon feu, Ex : brassez ou remuez donc le poële. Ils disent encore : brassez le chaudron, la poële, - pour - brassez ce qui est dedans; - et le rôti n'est pas brulé. Quoique cette manière de parler soit générale et a d'aussi bons effets journellement, on doit remarquer qu'elle est vicieuse.

Beurrée. sub. fém.

Ce nom, qui ne doit se donner qu'à (une tranche de [pain] sur laquelle on a étendu du beurre,) - s'applique encore, ici, à toute tranche de pain sur laquelle on a étendu quelque substance grasse, onctueuse ou liquide. Ex : Une beurrée de graisse, de crème, de confiture.

Beurrer. V. actif. Il n'est pas du tout françois.

Il signifie ici - étendre du beurre sur... Ex : beurrezmoi du pain. Prenez garde de vous beurrer les mains. On dit même, beurrer de la graisse, des confitures \&c. Beurrer se trouve dans le Dictionnaire de Danet ${ }^{(10)}$

Comfortable. adj. des 2 genres. La lang[u]e fran $\approx$ çoise n'a pas un semblable mot; il est entièrement anglois. C'est un adjectif à la mode et qui signifie tout ce que l'on veut. Voici ses principales acceptions. $1^{\circ}$ - C'est une nouvelle comfortable, - consolante.

$2^{\circ}$ - Tems comfortable, journée comfortable, - agréable.

$3^{\circ}$ - Mener une vie comfortable, - douce, contente.

$4^{\circ}$ - Liqueur comfortable, - qui fait plaisir, agréable à boire.

$5^{\circ}$ - Une nourriture, un mêt $[s i c]$ comfortable ${ }^{(11)}$, - qui fortifie, confortatif.

Capuche. sub. mas. ${ }^{(12)}$ C'est le mot usité dans

la Campagne pour un chapeau ou bonnet de femme.

Ex. Une capuche de paille, de castor. Apporte la capuche de ta mère. Où est la capuche de ta sœur?

Couette. sub. fém. C'est la queue de cheveux que portent les hommes. Faites-moi la couette. Il n'est pas françois.

Couette se dit il est vrai pour (lit de plume) mais alors il est masculin. Ex. On me donna un assez bon couette. Nous ne faisons pas usage de ce mot dans ce dernier sens.

Cree ou Cris. Nom d'une nation Sauvage du

Canada, dont on se sert dans cette phrase : malin comme un $\underline{\text { Cree. }}$ (on prononce toujours Cris.) 
Chienneter. V. n.

On l'emploie à tort au lieu de chienner, (faire des chiens.) Ma chienne a chienneté, pour chienné.

Côte. sub. fém. Ce mot, qui signifie le penchant d'une montagne, d'une colline et les rivages de la mer, Ex : les côtes de l'Océan \&c., ne sauroit s'appliquer à toute éminence, hauteur et élévation; et c'est un des abus que nous en faisons.

Nous nommons aussi côte, dans nos campagnes, une rangée de terres concédées, ou une suite d'habitations. Ex : La côte de la Visitation. Un habitant de la côte Saint Luc. Je viens de la Grand'côte.

Côtes, pluriel signifie campagnes dans cette phrase très usitée, il va courir les côtes. Il a fait sa fortune à courir les côtes. Ceci se dit de certains Merciers qui vont vendant des marchandises de campagne en campagne.

On dit encore d'un vagabond, il court les côtes; c'est un coureur de côtes; pour exprimer qu'on ne lui connoit pas d'azile fixe. On dit en françois, dans ce même sens, c'est un coureur.

Chatoner [sic]. V. n. Il s'emploie ici au lieu ${ }^{(13)} \mathrm{de}$ chatter, (mettre bas des chats.) La chatte est au moment de chatonner. Elle a chatonné cette nuit, - pour - chatté.

Cavalier. sub. mas. pour Amant. sub. mas. (celui qui aime.) Ex : voilà votre Cavalier qui passe. Cette petite fille parle déjà d'avoir des Cavaliers.

Cantine. sub. fém. ou Cannevette. sub. fém.

Nous donnons également ces deux noms, à ce que les

François appellent simplement Cantine. C'est un (petit coffre divisé par compartimens, pour porter des bouteilles et des phioles en voyage.)

La Cantine est à l'armée (le lieu où l'on vend du vin et de la bière aux soldats. $)^{(14)}$ Les Canadiens ont encore appliqué ce nom à tout cabaret ou taverne. Il est à la cantine.

Cantinier. sub. mas. C'est celui qui à l'armée tient la cantine, et le mot est françois dans cette seule acception. On doit donc dire le Cabaretier, le Taver nier, et non pas le Cantinier, quand on veut parler d'un 
homme qui vend, ailleurs qu'à l'armée, de la boisson au détail.

Cantinière. sub. fém. Synonime Canadien de

Cabaretière, tavernière. Il n'est ${ }^{(15)}$ usité dans la langue françoise.

Canotée. sub. fém. On entend par ce mot :

$1^{\circ}$ Tout ce qu'un canot peut contenir, un canot plein.

Ex : une canotée de volailles, de sucre. Il vient de partir une canotée d'enfans, ou plein un canot d'enfans.

$2^{\circ}$ Dans le commerce des pelleteries, la canotée est la charge d'un canot d'écorce. J'ai monté à Michillimaki nac avec six canotées ou canots; car dans ce sens ces mots sont synonimes. - La charge d'un canot employé dans ce commerce est de 60 pièces ou paquets de marchandise, du poids de $90 \#$ [= livres] à $100 \#$ chacun, et de $1000 \#$ pesant de munitions de bouche. C'est ce qui s'appelle un canot ou une canotée.

Canoteur. sub. mas. Canoteuse. sub. fém.

Qui aime à canoter. Qui sait bien canoter. Il se dit plus particulièrement des enfans.

Canoter. V. n. - pour - Conduire un canot. Ex. Il sait bien canoter.

On dit encore, les enfants ont canoté tout le jour; il aime bien à canoter; ; $^{(16)}$ allons canoter; et ce verbe sig $\approx$ nifie alors - aller, se promener en canot.

Cordeaux. plur. sub. mas. Courroies. sub. fém. plur. ou Guides. sub. fém. plur.

Mots employés le plus communément au pluriel, pour exprimer (les longues rênes attachées à la bride d'un cheval attelé,) et pour le conduire. Guides est le mot propre; et l'on doit dire : donnez-moi les guides, et non pas les cordeaux, ou les courroies.

Câdre. sub. mas. Mot dont on ${ }^{(17)}$ se sert assez inconsidé rément au lieu ${ }^{(18)}$ de Tableau ou Peinture; - voilà un beau câdre, pour une belle peinture, un beau tableau. Le cadre d'un tableau c'est sa bordure.

Désapointer. v. actif. Vieux verbe qui étoit employé dans ce sens : (ôter les appointemens à un homme de guerre. Ex : on a désapointé plusieurs Offi ciers réformés.) Il n'est plus d'usage. Cependant il 
commence à être beaucoup en vogue ici, c'est un mot favori. Il est toujours accompagné de l'auxiliaire être. Ex : J'ai été grandement désapointé aujourd'hui, je n'ai pas trouvé Madame chez elle. Je devois recevoir hier $£ 100$, mais combien j'ai été désapointé, ${ }^{(19)}$ en apprenant que mon débiteur étoit enfui! \&c. En un mot, un homme à qui l'on a manqué de parole, qui éprouve quelque contres tems, ou quelque traverse, - qui manque son coup, qui ne vient point à bout de son dessein, qui est trompé dans son attente, ou qui échoue dans ses plans ou ses projets, est dans tous ces cas désapointé. Toutes ces acceptions sont purement Angloises.

Démancher. V. actif. (ôter le manche.)

Outre sa vraie signification, on lui en donne d'autres ici. Ex : -

$1^{\circ}$ Démettre, - j’ai le bras démanché, le doigt démañ ché. - pour - démis.

$2^{\circ}$ J'ai fait démancher mon écurie ${ }^{(20)}$, ma maison, pour exprimer ou que je les ai fait jetter bas, ou que je n'en ai fait défaire qu'une partie.

$3^{\circ}$ Mon horloge est démanchée, c'-à-d., ou qu'elle est démontée, ou qu'elle n'est plus réglée, plus en ordre. $4^{\circ} \mathrm{Sa}$ voiture s'est démanchée entièrement en versant, au lieu de s'est brisée. Il est ici réfléchi. Je vous prêterois volontiers ce fauteuil, mais vous voyez qu'il est tout démanché - (brisé, cassé.)

$5^{\circ} \mathrm{Ma}$ robe étoit devenue trop juste, je l'ai déman chée, - pour décousue. Je serai contraint de faire déman cher cet habit, c'-à-d. découdre, ou défaire. $6^{\circ}$ Qui a donc démanché le lit? Ne vous asseyez pas sur ce lit, vous le démancheriez, - au lieu de dire, défait, déferiez.

Débarquer. V. a. \& n. (Sortir, descendre d'un vaisseau.) Terme de marins ${ }^{(21)}$ qu'on emploie dans sa signification ordinaire, et pour $1^{\circ}$ descendre de voiture. Ex : Monsieur B... débarquoit de sa caléche, comme je passois devant chez lui; ou simplement Il débarquoit, comme je passois. Allons, vite, débarque. $2^{\circ}$ descendre de cheval. Il est débarqué de cheval, \& simplement je débarquerai chez toi, en passant. 


\section{$3^{\circ}$ descendre ou sortir de bateau, de canot. -}

Ces manières de parler sont sans doute impropres, et il seroit très facile de s'en corriger.

$4^{\circ}$ On dit encore assez mal-à-propos : débarquez

le fouet, de la caléche. Avez-vous débarqué(2) mon porte-manteau de dessus le cheval? On seroit aussi bien entendu, si l'on disoit, - ôtez le fouet de la voiture, entrez le, - descendez mon porte-manteau, ${ }^{(2)} \&$ c. et l'on parleroit au moins notre langue.

Décent. - te. adj. -

Beaucoup de nos puristes du bon-ton disent avec satisfaction : Monsieur $\mathrm{D}$. a eu des funérailles très décentes, pour honorables. Cette maison est bien décente, c'-à-d. belle, bonne, bien meublée. Cet ${ }^{(24)}$ homme est bien décent dans son habillement, ou en françois - il est vêtu bien proprement. Il est extrêmement ou très décent chez lui, ou dans sa maison, pour exprimer que cette personne est bien polie au monde, - qu'elle reçoit bien la compagnie.

Il est aisé de voir que toutes ces expressions sont angloises.

Dirigé. part. passé. C'est un mot de Cour, que

le verbe être régit toujours et qui n'est jamais françois dans l'emploi qu'on en fait dans cette phrase. Un secretaire écrit : je suis dirigé par son Excellence de vous informer que 8 c. - Il signifie, je suis enjoint par... j’ai ordre de... Il est anglois dans ce sens.

Dégobillage. sub. mas. pour Dégobillis sub. mas.

(C'est un mot bas, qui ne ${ }^{(25)}$ se dit que du vin et des viandes vomis, et qu'on a pris avec excès) Ex. Cela sent le dégobillis, et non le dégobillage.

Dégobiller, V. actif. Synonime de vomir, est aussi un terme bas. Il est françois.

Décaniller. V. n. Il signifie s'enfuir préciz

pitamment, se retirer ${ }^{(26)}$ promptement, - sortir en hâte de quelque lieu. Ex : Ces enfans décanillèrent bon train, quand ils surent que vous veniez à eux. A peine vous vit-on, que toute la maisonnée décanilla. On dit faire décaniller quelqu'un, dans le même sens. Si j'étois de vous, je ferois ${ }^{(27)}$ décaniller bien vite 
ces gens de votre maison.

On se sert aussi de ce verbe absolument. - Allons, qu'on décanille à l'instant. ${ }^{(28)} V$ ite, décanillez, bande de coquins. -

par Exemple. Façon de parler adverbiale usitée $^{(29)}$ dans le langage famillier. Ex : Oh, par exemple, c'est trop mentir! - au lieu de - Oh, pour le coup. Cette expression n'a souvent aucun sens distinct, comme lorsqu'elle est précédée de ${ }^{(30)}$ mais. Ex : je ne puis vous prêter mon cheval, mais par exemple ma voiture est à votre disposition.

Ebrayer. V. a. Donner des coups de corne, de manière à faire mal ou dommage. Il se dit du bétail à corne[s]. Ex. La vache t'ébrayera quelque jour, tu la maltraites trop. Elle a ébrayé l'enfant.

On dit au figuré, d'un homme qui a été bien rude $\approx$ ment battu, - il s'est fait ébrayer; il l'a ébrayé.

Ebrayer a quelquefois Corner pour synonime; mais il faut pourtant remarquer qu'il y a cette différence entre les deux verbes - qu'Ebrayer entraine toujours avec lui l'idée de blessure, - tandis que corner a une signification moins mauvaise, et s'applique seul au jeu que font souvent les vaches en se heurtant de leurs cornes. Voyez Corner.

Etrivant. te. sub. \& adj. pour - qui fait des

agaceries, qui tourmente. Ex. Finis donc, étrivante. C'est le plus grand étrivant que je connoisse. Elle est étrivante au delà de ce qu'on peut dire. Oh! qu'il étoit étrivant, quand il étoit petit!

Etriver. v. a. pour Agacer, Tourmenter par

ses jeux \& . Ex : Ne m'étrivez donc plus. Il l'a fait étriver tout le jour.

Etrivant \& Etriver ne sont françois ni l'un ni l'autre.

Ecrapoutir. v. a. Ecrapoutiller. v. a. Ces

verbes qui sont synonimes, signifient - aplatir, écraser, briser par le poids de quelque chose, ou par quelque effort. Ex : Je t'écrapoutirai le nez d'un coup de poing, si je me fâche. Si je vais à toi je t'écrapoutille. Il a mis le pied sur cette arraignée, cette grenouille et les a écrapoutillées. On dit d'une personne qui en a écrasé 
une autre de ses coups, - il l'a écrapouti, ou écrapoutillé comme un crapaud.

En joignant le pronom personnel à ce verbe, on dit alors : il s'est écrapouti, ou écrapoutillé la main avec le marteau. Nez, - doigt écrapoutillés - du part. passé.

Embarquer. v. a. \& n. On fait de ce verbe le même abus que de Débarquer. Ex. Si la voiture est prête, embarquons vite, - pour - montons dedans. Soyez prête à embarquer à 2 heures, c.-à-d. à monter en voiture. On embarque également une cassette, des effets dans une voiture, pour mettre dedans. Si vous voulez embarquer, Monsieur votre cheval est sellé et à la porte.

Parler ainsi n'est pas parler françois.

Empois. sub. mas. (Espéce de colle faite avec de

l'amidon, et dont on se sert à rendre le linge plus ferme.)

Ex : Empois ${ }^{(31)}$ blanc. Empois bleu. Mettre le linge dans l'empois.

On donne ${ }^{(32)}$ ici le nom d'empois indifférem $\approx$ ment à l'amidon et à l'empois même. La définition de l'amidon suffira seul [sic] pour faire voir que ce n'est pas parler avec justesse. L'Amidon est (une certaine pâte qui est faite de fleur de froment séche, et qu'on délaie pour en faire de l'emplois $[s i c]$.) -

Ecourer. V. a. Employé pour Faire soulever le

cour, faire mal au coeur. Ex : La moindre chose l'écoure. Il est facile à écœurer. Ce ragout m’a écouuré. Cette femme est si mal-propre qu'elle en écoure.

Ecceuré. ée. sub. c'est un écceuré du premier ordre, c'-à-d. un dégouté, difficile. Madame fait bien l'écourée, dans le même sens.

Eccurant. te. adj. Il s'emploie pour malpropre.

Ce couteau est réellement écoeurant. Vos mains sont écœurantes, allez les laver. On dit encore d'une personne sale \& malpropre, - quel écourant morceau!

On l'emploie aussi comme substantif. Quel est cet écœurant, (malpropre.)?

Ecolter. V. a. s'Ecolter. V. a. pour - se décou vrir l'estomac avec indécence. Il s'applique particulière $\approx$ ment aux femmes. - Ex : Qui vous a écolté de la sorte? Elle est toute écoltée. Que je vous voie vous écolter de la sorte. - Voyez Ebrâiller. 
Ebrâiller. V. a. pour se Débrailler. V. a.

(se découvrir l'estomac avec indécence.)

Ce verbe a la même signification qu'Ecolter, mais il s'applique aussi bien des hommes que des ${ }^{(33)}$ femmes.

Ex : Elle est toujours ébrâillée. Il est revenu tout ébrâillé.

On dit aussi substantivement d'une fille indécemment mise, et d'une fille publique, - quelles ébrâillées! Ce sont des ébrâillées.

Se débrâiller ${ }^{(34)}$ est le seul mot que la lang[u]e permette. -

Flasque. sub. mas. (un paresseux. Dictionnaire de Boyer.)

On l'emploie ici dans ce sens \& de plus dans celui de Peu reux. Il a manqué de courage dans cette occasion, c'est un flasque. La plus petite fille est moins flasque que lui.

Flasque. adj. des 2 genres (qui est sans force, sans

vigueur.) Cette acception qui est la seule françoise n'est pas ${ }^{(35)}$ usitée ici; mais on se sert de ce mot au lieu de mola lasse, dans ces phrases. Cette indienne est bien flasque.

Cette soie est flasque, je n'en veux pas.

Mots ${ }^{(36)}$ bas et populaires.

Flasquer. V. n.

Il signi[fi]e avoir peur. ${ }^{(37)} \mathrm{Ex}$ : -

Il a flasqué hier. Un enfant peut le faire flasquer, lui faire peur. Mot populaire.

Flasquer. V. a. Qui n'est employé qu'à l'infinitif

et dans cette seule phrase, - des fers à flasquer, (à repasser le linge.) Il n'est pas françois.

Fesser. v. a. (Fouetter, frapper sur les fesses

avec des verges ou la main.) Il ne se dit que d'un enfant. Ex. Fessez cet enfant.

Cependant on dit ici, tous les jours, d'un homme qui a été fouetté sur le dos par le bourreau, - il a été fessé. Le bourreau l'a fessé impitoyablement. La Cour I[']a condamné à être fessé en plein marché. C'est parler improprement; on doit dire fouetté.

Fenouil. sub. fém. pour - Fenouil. sub. mas.

(plante aromatique.) Nous disons, il y a de la fenouil dans ce tabac; il faut dire du fenouil, car il est masculin. Nous donnons à fenouil le synonime fenouillette, qui n'est rien moins que tel. Voyez ce mot. 
Fenouillette. sub. fém. Nous employons ce mot pour fenouil, plante aromatique; mais la fenouil $\approx$ lette est proprement (une espéce de pomme qui a le gout du fenouil.) On ne peut donc pas dire : Il y a de la fenouillette, (pour du fenouil,) dans le tabac que vous me présentez.

Fàro. sub. mas. Gallant - petit-maitre. Il se dit des gens de la campagne. Ex : C'est un farro. Il fait le fâro. Il est beau comme un faro de campagne, en parlant d'un homme extrêmement recherché dans ses habillemens.

Fàrôder. V. a. ${ }^{(38)}$ C'est faire le fâro, ou la cour aux Dames. Ex : Qui farôdez-vous? Il y a longtems qu'il fârôde Mademoiselle N., il devroit bien l'épouser. Il commence à farôder. Eh quoi, ne se mêle-t-il pas de farôder, à son âge!

Fine-boutique. sub. fém. Ce terme désigne un matois, un rusé, en même tems qu'il se dit d'un $\underline{\underline{\text { diotiot}}}$, d'un esprit borné. Exemples : $1^{\circ}$ - Ne vous y jouez pas, c'est une fine-boutique. Si vous traitez avec lui, ${ }^{(39)}$ soyez bien sur vos gardes; car c'est une fine-boutique, et vous pourriez être sa dupe. (gravement.) $2^{\circ}$ - Ne me cite donc jamais l'opinion de P.; en voilà t-il pas une fine-boutique! C'est une fine-boutique, pour se mêler de cela! Oh! la fine-boutique! (ironiquement.) On sent bien que le ton dont on prononce ce mot, détermine ${ }^{(40)}$ le sens qu'on veut lui donner; et c'est ainsi que matois ou fin-matois, rusé ou rusé-compère, [sont] synonimes de fine-boutique.

Fignoler. v. n. On dit d'un jeune homme qu'il fignole, qu'il commence à fignoler, quand il se met à faire des dépenses d'éclat, soit en habits, voitures \&c. \& à faire le galant. Ex : - C'est trop fignoler, tu verras bien vite la fin de tes écus. Il n'a pas le sou, et il voudroit fignoler.

Fignoleur. sub. mas. Celui qui fignole. Ex. C'est un fignoleur. Il devient fignoleur.

Flamblant $^{(41)}$. te. adj. Cet adjectif n'est pas françois. On lui donne ici, ${ }^{(42)}$ dans l'usage qu'on en fait, la signification de brillant, reluisant, et il est presque toujours accompagné de l'adjectif neuf, neuve. Exemples : 
Il avoit hier un habit tout flamblant neuf. Un chapeau flambant neuf. Il m'a donné un couteau tout flamblant neuf. Une épée toute flambante neuve. ${ }^{(43)}$ Ces expressions sont sans doute figurées et servent à exprimer le lustre de l'habit et du chapeau, ${ }^{(4)}$ le poli de l'épée et du couteau; aussi ${ }^{(45)}$ dit-on en bon ${ }^{(46)}$ françois, dans ce même sens, ${ }^{(4)}$ des épées flamboyantes, en allusion au poli de ces armes.

On dit d'une personne habillée tout ${ }^{(48)}$ en neuf, qu'elle est flamblante. Comme il est flamblant! Te voilà tout flamblant. Il est tout flamblant nud, pour entièrement nud; je te passerois mon épée toute flamblante a ${ }^{(49)}$ travers le corps, pour toute entière, ou j'usqu'à la garde, sont encore ${ }^{(50)}$ des manières de s'exprimer très familières. -

Finition. s. f. pour fin sub. f. - à la finition de la Messe. ${ }^{(51)}$

Ginseng. sub. mas. (plante.) La chute de cette

branche de commerce en Canada a donné naissance à cette manière de parler proverbiale, - tombé comme le Ginseng; c'-à-d. tout-à-coup, et sans espoir apparent de se relever. C'est pourquoi l'on dit d'un homme qui n'est plus en faveur, qui est tombé dans le discrédit, qui n'a plus de popularité, \&c. et qui a éprouvé ces inconstances inopi nément, - il est tombé comme le ginseng.

Giboulée. sub. fém. (petite pluie froide, qui tombe à plusieurs reprises.) C'est dans ce cens qu'on le dit ici de la nége. - Nous aurons aujourd'hui quelques giboulées, ou quelques giboulées de nége. Laissez passer la giboulée. Ce n'est rien que cela, ce ne sera qu'une giboulée. Il équivaut à petite bordée, que nous employons aussi. Voyez Bordée.

Gâ. sub. mas. Ce mot signi[fi]e à la campagne - $\underline{\text { un }}$ jeune garçon. Ecoute, écoute, mon gâ. viens ici, mon gâ. Il est le plus souvent précédé de petit. Ex. Où est le petit gâa ? Je vous enverrai mon petit gâa. Vous donnerez ceci au petit gâ.

Guide. sub. fém. (Longue rêne attachée à la bride d'un cheval attelé.) Voyez Cordeaux \& Courroies.

Gavache. sub. ${ }^{(52)}$ mas.

Ce mot, qui dans sa vraie acception signifie Coquin, misérable, est employé parmi nous comme synonime 
de poltron. On le fait même féminin, et l'on dit : c'est une vraie gavache, une grande gavache.

Gueusaille. sub. fém. Gueusasse. sub. fém. (Canaille.)

Ex : Ce n'est que de la geusasse [sic]. C'est la plus franche gueu sasse que je connoisse. Il ne loge chez lui que de la gueusasse. Retire-toi de moi, gueusasse, $c^{\prime}-\grave{a}-d$. coquin. Point de ta gueusaille ici. On se sert également de ces deux mots, quoique gueusaille soit le seul françois.

Gouliâ. sub. mas. Ce substantif répond ici aux adjectifs françois - gouliafre \& goulu (qui mange avidement \& malproprement.) Ex. Il mange comme un gouliâ, c'-à-d. beaucoup. C'est un gouliâ, un vrai gouliâ. On dit en fran çois dans ce cens, c'est un homme extrêment [sic] goulu; c'est un vrai gouliafre.

Gouliâ se dit aussi d'une femme. Ex. Elle a un appetit dévorant, c'est un vrai gouliâ que cette servante.

Georges. sub. fém. plur. Employé ici pour Orges.

sub. fém. plur. dans cette phrase proverbiale - faire ses georges, pour dire faire son profit, faire bien ses affaires. Orges, ${ }^{(53)}$ est françois et usité dans ce même sens, - il y a fait ses Orges. Il est du stile familier.

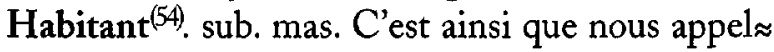
lons notre agriculteur, ou laboureur. Ex. Il y avoit bien des habitans au marché. Je suis habitant. Ce n'est pas un homme de la Ville, c'est un habitant, ou un homme de la Campagne. - On ne connoit point ici de paysans, ce sont tous des habitants. - Fils d'habitant.

Habitante. sub. fém. Celle qui habite la Campagne, qui cultive la terre. On voit bien à son teint qu'elle est ${ }^{(55)}$ habitante, ou une habitante.

Hausse. sub. fém. Sorte de vêtement en usage chez nos Sauvages, qui sert à couvrir la jambe. On le nomme aussi Mitasse. sub. fém. Ex. Voilà de jolies hausses ou mitasses. Hausses de cuir. Mitasses d'étoffe. Ce dernier est françois.

Houiller. V. a. Verbe trivial usité parmi le peuple pour changer ${ }^{(56)}$, troquer une chose contre une autre. Il s'ap plique plus particulièrement à l'échange de chevaux. Ex : Veux-tu houiller ton cheval, ou absolument, - veux-tu ${ }^{(57)}$ houiller? Si tu veux payer chopine, je houillerai avec toi. Houillons, 
houillons vite. Il ne doit pas s'employer dans ce sens, mais il est françois dans les phrases suivantes.

Ex : - Je suis houillé de ma femme; j'en suis las, dégouté. Je suis houillé de mon hôte; il me déplait, il me pèse sur les épaules. Je suis houillé de la vie, las de vivre. Ce verbe ${ }^{(58)}$ est quelquefois refléchi, - il se houilla de vin, - pour - se gorgea de vin.

\section{Improuver. v. $\mathrm{n}$.}

On dit fort généralement dans nos Villes - ce jeune homme improuve à vue d'oil, pour exprimer qu'il fait des pro grès rapides, ou qu'il se perfectionne bien sensiblement. Cette terre a beaucoup improuvé, depuis 2 ans, pour s'est beaucoup ameliorée. Ces acceptions sont prises de l'Anglois -

Improuver est un verbe françois il est vrai, mais son sens propre est désapprouver. De plus il est actif - Ex : Le Roi improuva très fort la conduite de ce général.

Immatériel. elle. adj. Autre mot françois auquel on donne des significations qu'il n'a pas. Ex : On dit souvent, et surtout au Barreau, c'est une faute immatérielle, - pour - légère, peu grave. Tout ce que Monsieur dit est absolument immatériel, $c^{\prime}-\grave{a}-\mathrm{d}$. inutile, ou hors du Sujet, ou d'aucun poids. Ces phrases sont de purs anglicismes.

Immatériel, elle, adj. est un terme ${ }^{(59)}$ didactique, qui signifie en françois (qui est sans mélange de matière.) Ainsi l'on dit les substances, les formes immaterielles.

Inconsistant. te. adj. C'est l'adjectif Anglois Inconsistent, qu'il étoit si facile de franciser! Il signifie incompatible, contraire, contradictoire.

On dit aussi, c'est bien inconsistant de votre part de... pour - c'est bien inconsidéré de votre part que de... Il est du haut ton.

Jument. sub. fém. Terme du Commerce des pelleteries. C'est une caisse de fusils. Voilà une jument qui pése beaucoup. Cette jument m'a meurtri l'épaule en la portant.

Juifrèsse. sub. fém. au lieu de Juive, qui est beaucoup plus rarement employée. C'est une Luifresse, une Juive, une femme juive.

Il ne doit pas se dire. 
Jouquer. V. n. pour Jucher. V. n. On ne l'em $\approx$

ploie guère qu'avec le pronom personnel. Ex : Les poules

se jouquent à cette heure, pour se juchent à cette heure.

Où cet homme s'est-il allé jouquer! (s'est-il allé jucher.)

pour exprimer - quel séjour il a pris! où va-t-il se mettre! en marquant de la surprise.

On dit ici - les poules sont jouquées, plus communéঞ ment que - les poules se sont jouquées ou juchées.

Jouquoir. sub. mas. pour Juchoir sub. mas.

Joli-cœur. Ce mot, qui ne va jamais qu'en

compagnie des verbes - s'appeller, se nommer, s'emploie tous

les jours dans les phrases suivantes : ${ }^{(60)}$ à vous voir dispo $\approx$ ser de ces fruits, je vois que je me nommerai joli-cœur; c'-à-d. je vois qu'il n'en restera pas pour moi. Ils man gèrent à eux seuls toutes les pommes, et je m'appellai joli-cœur. Tout le monde se servit, et je m'appellai jolicoeur, c'-à-d. je n'eus rien.

Licher. V. a. pour lécher V. a. (passer la langue

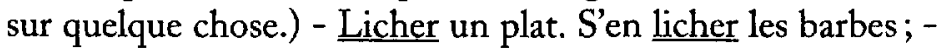
au lieu de lécher.

On dit familièrement - d'un fils dépensier - il en a bien liché, il en lichera bien à son père.

Lichefrite. pour Lèchefrite. sub. fém. (ustencile de cuisine[)].

Légerte. féminin de Léger, re. adj.

L'emploi de légerte pour légère, est une de ces fautes qui se commettent ici journellement. Ex. La compagnie légerte. C'est une fille bien légerte. Il a la tête extrêmement légerte.

Loucheur. euse. adj. pour Louche. adj. des 2 genres

Ex. Cet homme est loucheur, pour louche.

Il est aussi substantif : Ex. je ne veux point d'une $\underline{\text { lou } \approx}$ cheuse. Un loucheur. - On ne sauroit employer louche, dans ces deux phrases, parce qu'il n'est jamais substantif; mais il faudroit dire : Je ne veux point d'une femme louche. Un homme louche.

Notre loucheur a pour synonimes Bicleux \& Virel'œil. Voyez ces mots.

Mouiller. V. n. On l'emploie ici presque ex $\approx$ clusivement à celui de pleuvoir, comme : il mouille, il 
a mouillé, il mouillera; au lieu de - il pleut, il a plu, il pleuvra. Dans ce cens, mouiller ne peut absolu $\approx$ ment pas se dire.

Mouiller est un verbe actif, qui ne s'emploie jamais en françois, que pour marquer l'action de tremper, $\underline{\text { hu}} \approx$ mecter, rendre humide. Ex : la pluie a mouillé les chemins. Mouiller un linge dans l'eau. Mouiller l'ancre, ou simplement - mouiller; c'-à-d. jetter l'ancre pour arrêz ter le vaisseau.

Marier. V. a. pour Epouser. V. a. Dans ce nouveau sens, que la langue françoise ne sauroit $\mathrm{ad} \approx$ mettre, marier est déjà bien en vogue, et l'on dit dans la bonne compagnie même - Monsieur marie mama selle; cela veut dire que Monsieur épouse Mademoi selle. Nicolas a marié Lisette, ou a épousé Lisette. C'est employer, on ne peut pas mieux, ${ }^{(61)}$ des mots françois à parler anglois.

Mitasse. sub. fém. Mot ${ }^{(62)}$ Sauvage reçu dans la langue françoise. Nous disons aussi Hausse. Voyez ce mot.

Mondaine. adj. pour Mondée. part. Ex : de l'orge mondaine, pour de l'orge mondée. Mondaine est plus usité dans la campagne que mondée, qui y est aussi connu.

Micoine ou Micouène. sub. fém. Petit vase de bois qui sert de cuiller à nos Sauvages. Une grande micoine. une micouène de bois.

Méche. sub. fém. L'extrémité la plus déliée d'un fouet, et qui souvent est ${ }^{(63)}$ une ficelle rapportée. Ex : il a usé, il a perdu la méche de son fouet. Ce fouet n'a plus de méche. On doit employer - Claque..$^{(64)}$ Méche ou Pipe. sub. fém. Pour exprimer que la distance est grande d'un lieu à un autre, on dit : Oh! il y a une méche d'ici là. J'ai encore une bonne méche à faire. - Voyez Pipe.

Mécher. V. a. ${ }^{(65)}$

Ce verbe, quant au sens, répond au verbe françois Rosser. Ex : Si je vais à toi je te mécherai d'importance. Il méchoit son cheval sans pitié.

Mâle. sub. mas. Vêtement de tête de nos habitans; c'est un bonnet de laine tricottée. Ils lui donnent 
aussi le nom de Tuque. Où est mon mâle? As-tu vu mon mâle. Voyez Tuque.

Mistimus, en Mistimus. Mots employés pour bien, très bien. Ex. Ceci est écrit en mistimus. C'est ce qu'on appelle du mistimus, du bon, du parfait. Il a fait cela en mistimus, c'-à-d. très bien.

Naturel. elle. adj. On lui donne ici sou vent la signification de salubre, qui contribue à la santé. Ex : c'est un air bien naturel. Nourriture naturelle. Cette tisanne est très naturelle, - prenez-en. ${ }^{(66)}$

Nijon. sub. mas. $\mathrm{Il}^{(6)}$ répond ici au mot françois

Bout-d'homme. On lui donne aussi pour synonime - Bougon. Voyez ce mot.

Originer. V. n. Il n'est pas françois, quoique la

Magistrature même s'en serve ici, et qu'on ait tout lieu de croire qu'il ne vient point d'une source ignoble; car ce mot r'est d'usage que parmi nos gens comme il faut. Voici de leurs phrases : - Savez-vous d'où ce mot origine, - pour Savez-vous d'où dérive ce mot, ou quelle est l'étymologie de ce mot? Je ne sais d'où cet homme origine, - au lieu de dire, - je ne sais d'où il tire son origine, d'où il sort, quelle est son origine. D'où originez-vous? pour - de qui, ou de quel lieu sortez vous; - d'où ${ }^{(68)}$ tirez-vous votre origine?

Cette maladie - origine d'un amas d'humeurs; d'ou [sic] croyez-vous qu'origine cet abus? - pour provient (de provenir.)

Originer. V. n. C'est encore un de ces mots pris de l'Anglois, (to originate), et qu'on emploie dans le sens $\mathrm{de}^{(69)}$ - provenir, tirer son origine ou son étymologie, dériver. ${ }^{(7)} \mathrm{Ex}:-\mathrm{D}$ 'où faites-vous origiz ner ce mot? - pour - dériver, ou quelle est ${ }^{(7)} \underline{\text { l'etymologie de }}$ ce mot? Je ne sais d'où cet homme origine, au lieu de dire je ne sais d'où il tire son origine, ou quelle est son origine. D'ou [sic] originez-vous? pour - de qui ou de quel lieu sortez-vous, tirez-vous votre origine ? Cette maladie origine d'un amas d'humeurs; d'ou [sic] croyez-vous qu'origine cet abus ? pour provient.

Ordonné. ée. part. Autre mot de cour. Ex. Ie suis ordonné par Son Honneur ou Son Excellence de vous dire que..., pour - j'ai ordre de Son Honneur ou de Son Excellence de vous dire que... Le régiment est ordonné de se rendre à Quebec, pour, 
a ordre, a reçu ordre ${ }^{(72)}$ d'aller à Québec. Cette brigade est ordonnée pour Montréal, ou plutôt - cette brigade a reçu l'ordre de se rendre à Montréal.

Toutes ces phrases sont angloises.

Orignal. sub. On donne ici ce nom à l'animal sauvage

que les Naturalistes nomment Elan. Ex. Un Orignal. Des Orignaux. Une mère Orignal.

Office. sub. mas \& fém.

Ce mot, qui ne doit s'employer que dans ces phrases rendre de bons offices; c'est l'office d'un bon père de...; assister à l'office divin; j'ai tel office dans l'administration; le Saint-Office, ('inquisition.) et qui dans tous ces cas est masculin : ou comme désignant le lieu où l'on garde la vaisselle, le linge ou ce qui concerne la table, ou enfin les cuisines, - et qui alors est féminin; ce mot, répéterai-je, a cependant ici une acception plus étendue.

Ainsi, les archives d'un Notaire sont, en Canada, son office. Un Avocat vous envoie à son office trouver ses clercs. Un homme en place vous prie de passer à son office; il vous $y$ donnera audience à telle heure. Tout homme public, tout homme d'affaire $[$ sic $],{ }^{(73)}$ le marchand même ${ }^{(74)}$ - a son office : c'est le lieu où chacun de ces Messieurs travaille.

Paré. ée. adj. verbal pour Prêt. te. adj.

Ex : Etes-vous paré à partir, pour prêt à partir? Madame est parée à monter en vouture [sic], pour prête à monter \&c. Si vous êtes parés, partons. Quand vous serez parés, vous me ferez avertir; au lieu de prêts, dont on se sert aussi, ${ }^{(7)}$ moins généralement pourtant que de paré.

Payer. V. a. Il est de mode ${ }^{(76)}$ chez grand nombre de personnes de dire : j'irai, Monsieur vous payer demain une visite. Il faut pourtant que j'aille payer une visite à Madame. Voulez-vous ${ }^{(7)}$ bien lui payer mes complimens, mes respects? Dans ce sens, Faire - est trop vieux, sans doute! de plus il est françois! et Rendre n'est pas familier à ces gens.

Je me rappelle à ce sujet d'une phrase qui mérite place ici; je l'ai entendue [sic] proférer de mes propres oreilles. Elle est d'un homme qui malheureusement a fait trop de bruit ici, et dont les prétendues ${ }^{(78)} \underline{\text { lumières et le savoir }}$ étoient, à l'époque dont je parle, tellement préconisés, que 
les mettre en doute eût presque été un crime d'état. Il disoit, un jour, à la Chambre d'Assemblée, dont il étoit Membre : «On me verra toujours paré à payer le regard dû à l'objet en question...» $\mathrm{O}$ honte! Est il ignorance plus crasse que celle-là?

Pipe. sub. fém. Espéce de mesure de distance

chez nos habitans : c'est à peu-près trois lieues. Ex : Combien y a-t-il d'ici chez-vous? - Il peut y avoir cinq pipes, ou environ quinze lieues. On compte d'ici à la première habitation deux bonnes pipes, pour - six fortes lieues. Voilà une bonne pipe de faite.

Pipe s'emploie aussi simplement pour grande distance, et n'exprime pas alors ${ }^{(79)}$ de mesure fixe. Ex : Il y a une pipe, d'ici - là ! et une belle pipe! c'-à-d. - d'ici - là il y a loin, et bien loin! Au reste, le ton dont on prononce déter $\approx$ mine, le plus souvent, le sens différent de deux phrases semblables.

Dans ce dernier sens, la Pipe a ${ }^{(80)}$ pour synonime la Méche. Voyez ce mot.

Poudine \& Poutine. sub. fém. pour Pouding.

(Mêt ${ }^{(81)}$ anglois.)

Les françois en adoptant le mot Pouding des Anglois, n'ont rien changé à son ortographe, mais ils le prononcent Poudingue \& le font masculin. Ex. Un poudingue au riz. Un excellent pouding. Le pouding est un bon mêt anglois.

Prouvable. adj. des 2 genres. L'emploi de ce mot est très commun ici. Il signifie - facile à prouver.

Ex. Ce que j'avance est prouvable. C'est prouvable, pour - on peut en donner la preuve.

Il n'est pas françois.

Plaisant. te. adj. Employé mal-à-propos pour beau ou agréable, comme dans ces phrases : c'est un tems bien plaisant. Voilà une plaisante aprèsdinée. - Autre larcin fait à la lang[u]e Angloise.

Peter. V. (faire un pet. -) Outre ce sens, ce verbe en a d'autres ici. On l'emploie par exemple, pour $1^{\circ}$ Claquer; - ce fouet péte bien; faire peter son fouet. $2^{\circ}$ crever; - son fusil lui a peté dans la main. $3^{\circ}$ gercer; - il a la main toute petée; des lévres ${ }^{(22)}$ que le froid a fait peter. 
$4^{\circ}$ déchirer par quelque effort; son habit est tout peté; son pantalon étoit si juste, qu'en se courbant il a peté. $5^{\circ}$ fêler; le froid a fait peter tous mes verres, ou mes vitres. Ce verbe en un mot est d'un usage très commun chez les personnes peu attentives à parler la langue purement ; il n'est banni que de la bonne compagnie, et par décence seule. $6^{\circ}$ Crevasser. - La terre est toute petée. ${ }^{(83)}$

Quêter. V. pour Mendier ou Quémander.

Quêteur. euse. sub. qui ne doit se dire que d'une personne qui quête pour quelqu'un, est non seulement $\mathrm{em} \approx$ ployé ici dans ce sens, mais plus généralement pour Mendiant - \& Quémandeur. sub. -

Quitte. sub. mas. pour Acquet. sub. mas. c'-à-d. avantage, utilité. Ex. puisque vous voulez absolument vous y rendre aujourd'hui, vous aurez plus de quitte de passer par ce chemin que par l'autre. On a plus de quitte de s'accorder que de plaider. Quitte ne se peut ${ }^{(84)}$ nullement dire, il faut employer acquet dans ces phrases. Il suffit d'ailleurs de se rappeller que quitte n'est jamais qu'adjectif, pour se régler dans l'usage qu'on en peut faire.

Relevée. Sub. fém. (tems de l'après-dinée.)

Ce terme est employé ici dans sa vraie signification, mais comme il ne doit être d'usage que dans le Barreau, il seroit peut-être prudent de le lui restituer à jamais, de peur de chicane. Ex. à deux heures de relevée, pour de l'après-dinée.

Ramancher. V. a. On lui donne plusieurs significations, telles que $1^{\circ} \underline{\text { conter, }} \mathrm{ou}^{(85)}$ raconter avec diffusion. Ex. Que me ramanchez-vous? Je ne vous entends pas. Il me ramancha une histoire qui n'avoit ni queue, ni tête. $2^{\circ}$ raccommoder, réparer. - Ex. Il a bien ramanché ce chandelier. La caléche est bien ramanchée. Dans ce sens on emploie aussi Amancher. Voyez ce verbe.

Retraiter. V. n. - quand on l'emploie pour signiz fier faire retraite, se retirer. Ex. L'armée a retraité de deux lieues. Le Général se vit contraint de retraiter, au lieu de dire, qu'il a été obligé de faire retraite, de se retirer. C'est ${ }^{(86)}$ un anglicisme bien visible.

Ce verbe est aussi employé comme actif, mais alors il signifie $\underline{\text { Retraire, }}, c^{\prime}-\mathrm{à}-\mathrm{d}$. retirer par droit de parenté 
ou par droit Seigneurial, un héritage qui a été vendu; c'est alors un terme de pratique. On dit donc : j'ai retraité ou je vais retraiter cette terre. On trouve aussi dans les actes de concessions de terres de ${ }^{(8)}$ certains notaires, «avec le droit de retraiter ou de retrayer"; car les deux sont usités. Retraiter dans aucun de ces sens ne peut s'employer; il n'est pas lui-même françois. On doit dire, je vais retraire, j'ai retrait \&cc.

Retrayer. V. a. ou Retraiter V. a. pour faire un retrait. - Voyez retraiter.

Résous. participe du verbe Résoudre. Il est françois sans doute, mais comme tel (il n'est d'usage qu'en parlant des choses qui se changent, qui se convertissent en d'autres, et il ne se dit point au féminin - Ex. brouil lard résous en pluie); - au lieu qu'ici on l'emploie égale $\approx$ ment dans le sens de déterminé. Ex. Je suis résous à plaider. Je l'ai résous à venir avec moi. Il s'est enfin résous à se battre. \&c. On doit absolument dire : je suis résolu, je l'ai fait résoudre, il s'est enfin résolu à... Qu'a-t-il été résous dans cette assemblée? - au lieu de - qu'a-t-il été résolu?

Reintier. sub. mas. Mot employé dans nos

Campagnes pour les reins, quelquefois de l'homme, et tou jours des animaux. Ex. ce jeune homme a le reintier foible, ou est encore foible du re[i]ntier; terme badin dans ce sens. Du reintier d'un cochon on peut faire trois socs. Il lui a cassé le reintier à force de coups. Il n'est pas françois.

Rapiester ${ }^{(88)}$. V. a. pour Rapiécer (remettre des

piéces à un habit ou à du linge.) Ex. Je suis à rapiester ses chemises, pour rapiécer.

Et absolument : on n'est occupé qu'à le rapiester, pour dire - rapiécer le linge \&c. de telle personne; ou bien, je rapieste Monsieur.

Rapiesté, ée. part. Ex. habit rapiesté. Veste rapies tée. Il est tout rapiesté.

Souliers. sub. mas.

On donne ici le nom de souliers françois, aux souliers de cuir qui nous viennent de l'étranger et que les cordonniers manufacturent;

Celui de souliers de bouf, à ceux que nos habitans se font de la peau du bouf, et Celui de souliers sauvages, à ceux que nos Sauvages 
font de la peau des bêtes fauves qu'ils tuent, telles que le chevreuil, le caribou, l'orignal.

Sur. - préposition de lieu employée bien communément dans le sens de chez, et bien improprement. Ex : Je viens de sur mon oncle. Je vais sur le Notaire. Je dine sur ma tante. On doit dire chez mon oncle, shez le notaire, chez ma tante. Sarpidon. sub. mas. pour Tapageur. Ex. C'est un vrai Sarpidon. Quel Sarpidon, bon Dieu!

Suspect. te. adj. (Qui est soupçonné, ou qui mérite de l'être.) On le $\mathrm{e}^{(89)}$ fait signifier ici en outre, susceptible. Ex : elle est extrêmement suspecte, $c^{\prime}-\grave{a}-d$. susceptible, ou facile à blesser. vous êtes bien suspect, Monsieur.

On dit aussi substantivement : c'est une suspecte comme on n'en trouve guère; pour exprimer qu'elle est bien suscep $\approx$ tible, qu'elle se formalise d'un rien. Il n'est guère d'usage que parmi le peuple et n'est pas du tout françois dans ce sens.

Sur. ure. adj.

$\mathrm{La}$ langue françoise le fait synonime d'aigre et lui donne ce sens, quand on l'emploie pour marquer la qualité; c'est pourquoi on peut dire - des pommes sures, des fruits surs; l'oseille ronde est fort sure. Mais on ne sauroit dire sans blesser la langue, du vin sur, du lait sur, pour du vin, du lait aigres; parce que sur dans ces phrases exprime le vice de ces liquides. Il faut donc dire du vin aigre, du lait aigre.

Surir. V. a. pour Aigrir - (rendre aigre, faire devenir aigre.)

La chaleur surit ou fait surir le lait, pour aigrit le lait. Il n'est pas françois.

Sauvagesse. sub. fém. Femme Sauvage. - C'est une $\underline{\text { Sauvagesse; voilà une Sauvagesse. }}$ pour une Sauvage, une femme Sauvage.

Sieau. sub. mas. pour Seau. (Vaisseau à puiser ou à

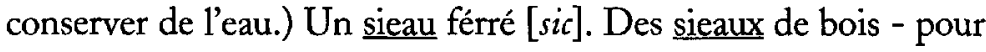
un seau, des seaux.

Tuer. v. a. Employé quelquefois pour Eteindre.

Ex : Tuez la chandelle. Avez-vous tué le feu?

Cette sorte de meurtre est très commune en Canada.

Trois fois heureux, mes Compatriotes, que la corde n'en soit pas la punition!

Tuque. sub. fém. ou Mâle. sub. mas. Vêtement de tête de nos habitans; c'est un bonnet de laine tricottée. Ex : 
Donne-moi ma tuque. Avez-vous des tuques à vendre. Voyez Mâle.

Train. sub. mas. Mot françois que l'on emploie à tort pour signifier : -

$1^{\circ}$ les occupations quotidiennes du ménage. - Ex. Mettezvous à votre train. Quoi! vous n'avez pas encore commencé

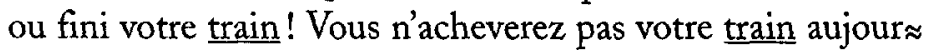
d'hui.

$2^{\circ}$ Ivre. Ex : Ils vont se mettre en train. Il est revenu en train à la maison. Ils sont tous en train. Il faut dire ivres.

Tricoler. V. n. Il se dit ici d'un homme ivre qui chancelle. Ex : Il tricole fortement. Je l'ai vu dans la rue tricolant de la belle sorte. Il n'est pas françois. Un Vendredi-Saint, Piron étoit ivre et dans les rues de Paris. Voltaire le rencontre; il va à lui, l'arrête et lui représente le scandale qu'il cause. - «Comment! lui dit-il, un jour comme celui-ci, - un Vendredi-Saint! vous, Piron, dans cet état! ah, fi donc!» - Oh! Oh! répond le poëte, si à pareil jour la Divinité succomba, l'humanité peut bien chanceler.

Trempe. adj. des 2 genres. Il n'existe pas dans la langue.

Ex. Il est tout trempe. Son habit est tout trempe. Elle est toute trempe de sueur, c'-à-d. qu'elle a beaucoup sué. On dit proverbialement - il est revenu trempe comme une soupe, $c^{\prime}-\mathrm{à}-\mathrm{d}$. très mouillé.

Toutes ces phrases seroient françoises si au lieu de trempe on disoit trempé, trempée.

Touche. sub. fém. Terme de fumeurs en usage dans ces phrases : Veux-tu fumer une touche. Viens fumer une touche. Après avoir fumé quelques touches, nous nous remîmes en route. C'est-à-dire fumer un peu $\&$ tout au plus une pipe pleine.

Tapin. sub. mas. une tape legère, un soufflet.

Il n'est pas françois.

Tapon. sub. mas. Mot que l'on emploie ici comme synonime de tas - paquet - touffe. Ex. Un tapon de laine. tapon de nége. La nége tombe en tapons, pour flocons. Un gros tapon de graisse.

On dit encore d'un petit enfant gros et gras, c'est un tapon, - c'est un tapon de graisse. 
Tinton. sub. mas. On dit ici - le tinton de la

messe sonne, - on sonne le tinton; pour exprimer (le son lent d'une cloche dont le battant ne touche que d'un côté.) Tinton n'étant pas françois, il faut parler ainsi : On tinte à la Paroisse; on tinte la messe, le sermon; ou absolument - la cloche tinte.

Tinter la grosse, ou la petite cloche, se peut aussi dire. Voyage. sub. mas. pour Voie. sub. fém. Ex. -

Deux sols sont d'ordinaire le prix d'un voyage d'eau, au lieu d'une voie d'eau. On appelle voie d'eau, et non pas voyage (les deux seaux d'eau que porte un homme.)

On doit dire ${ }^{(90)}$ aussi voie pour charretée, que nous employons exclusivement. Ex. Une voie de bois, voie de pierre, voie de sable, et non voyage ou charretée.

Vulgaire. adj. des 2 genres. Outre son vrai sens, on lui donne parmi le peuple ${ }^{(91)}$ celui de visible, évident. Ex. Il est vulgaire qu'il est midi. Il est vulgaire que tu perdras ton procès. Tout ce que vous dites là, Monsieur c'est vulgaire. ${ }^{(92)}$

Vire-l'œil. sub. pour Louche. adj. C'est un terme d'injure : - ôte-toi de là vire-l'œil ; point de vire-l'œil ici. Il se dit aussi par dérision, alors le ton détermine le sens qu'on veut lui donner.

Ses synonimes canadiens sont Bicleux et Loucheur. adj. Voyez ces mots. On doit remarquer qu'il se dit particulièrement d'une personne louche d'un seul œil.

Verbalement. adv. Il a plusieurs acceptions.

Ex. Il lui a parlé verbalement, c'-à-d. il lui a parlé

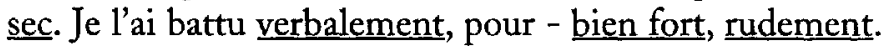
On dit d'un postillon, qu'il méne ses chevaux verbalement, ou qu'il méne verbalement, pour exprimer qu'il méne bien vite, ou bon train.

Cet adverbe est étranger à la langue françoise.

\section{Notes du manuscrit 2}

1. f[ini] raturé.

2. qui a plusieurs significations, et raturé.

3. ou surcharge et. 
4. Point et parenthèse fermante supprimés par nous.

5. du poisson et de la chair ajouté au-dessus de secher.

6. tout raturé.

7. et vire-l'œil, sub. ajouté dans la marge; signe de renvoi dans le texte.

8. tous raturé.

9. d surcharge deux $\mathrm{t}$.

10. Beurrer se trouve dans le Dictionnaire de Danet ajouté au crayon.

11. s raturé.

12. Donné comme tel dans le ms.

13. de celui de chatter qui seul est admis. Ex. La chatte pour raturé à l'exception de de chatter.

14. Mais raturé.

15. pas raturé.

16. il est raturé.

17. on ajouté.

18. au lieu surcharge pour raturé.

19. quand raturé.

20. soulignement enlevé sous écurie.

21. dont raturé.

22. $z$ raturé; accent aigu ajouté sur e.

23. pensez débar[quer] raturé.

24. te raturé.

25. ne ajouté dans l'interligne entre se et dit.

26. en hate raturé.

27. bien raturé.

28. à l'instant. ajouté au-dessus.

29. ainsi raturé.

30. mot illisible raturé.

31. I raturé dans emplois.

32. ainsi raturé.

33. aux et qu'aux remplacés par des et que des.

34. seul raturé.

35. fam[ilière] raturé.

36. Mots remplace Ces mots sont.

37. et il est populaire raturé.

38. \& n. raturé.

39. f[aites] raturé.

40. détermine surcharge decide raturé.

41. L'auteur écrit indifféremment flamblant et flambant dans cet article.

42. la signi[fication] raturé.

43. Il est tout flamblant nud, ou est entièrement nud. raturé. 
44. et raturé.

45. aussi surcharge et.

46. bon ajouté dans l'interligne.

47. dans ce même sens, ajouté.

48. e final raturé.

49. à surcharge au.

50. encore surcharge de ces faç[ons] raturé.

51. Article au crayon.

52. fém. raturé.

53. Il est du stile familier raturé.

54. e final raturé.

55. une raturé.

56. D'abord écrit échanger.

57. veux-tu surcharge allons raturé.

58. Ce verbe surcharge un mot illisible raturé.

59. un terme surcharge françois raturé.

60. Ex raturé.

61. D'abord écrit C'est on ne peut pas mieux employer

62. francisé du raturé.

63. souvent est surcharge est quelquefois raturé.

64. On doit employer - Claque. surcharge pour - Claque - écrit au crayon.

65. On l'emploie dans le même sens raturé.

66. C'est une acception prise de l'Anglois. raturé.

67. Il remplace $\mathrm{Ce}$ mot raturé.

68. d'où ajouté.

69. qu'on emploie dans le sens de surcharge que ceux qui l'emploient veulent faire signifier raturé.

70. Faisons raturé.

71. son raturé.

72. a ordre, a reçu ordre surcharge des mots illisibles raturés.

73. ou qui croit même en avoir raturé.

74. d surcharge $\mathrm{t}$ dans marchand; même surcharge \&c. l'artisan enfin raturé.

75. mais raturé.

76. de dire raturé.

77. vous ajouté.

78. prétendues ajouté.

79. alors ajouté.

80. Accent grave raturé.

81. Mêt dans les deux occurrences.

82. petées raturé.

83. La ligne entière avait d'abord été écrite au crayon. 
84. peut ajouté.

85. conter, ou ajouté.

86. donc raturé.

87. quel[ques] raturé.

88. Le verbe avait d'abord été écrit rapiéceter.

89. le surcharge lui raturé.

90. doit dire surcharge emploie raturé.

91. Virgule supprimée.

92. Point-virgule suivi de c'est raturé. 


\section{P 1.Verreau 67 No}

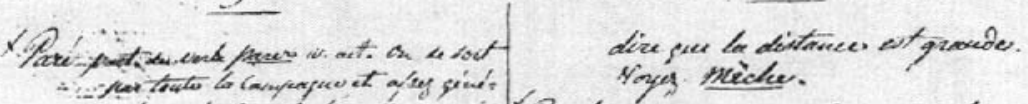

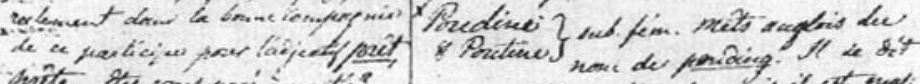

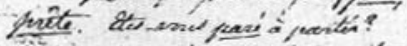

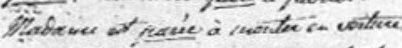

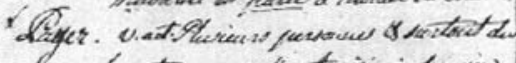

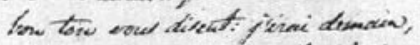

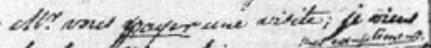

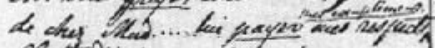

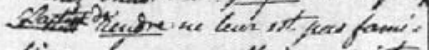

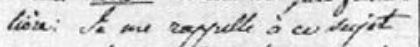

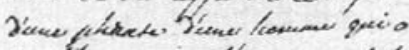

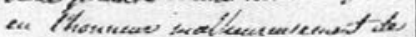

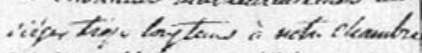

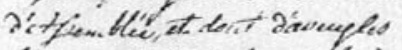

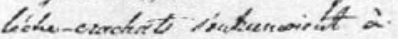

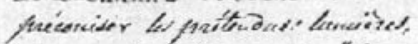

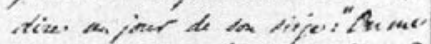

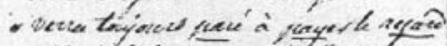
"dá alobirta sucition." Ruploi.

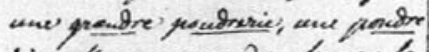
siv sfremes quaral en hyeer ha

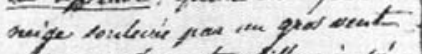

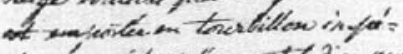

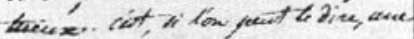
bricresgres der mejp.

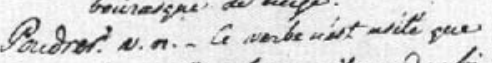

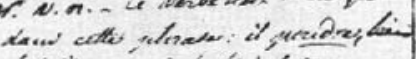

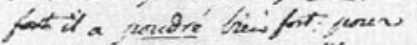

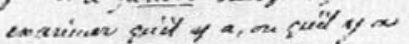

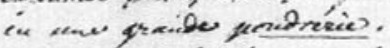

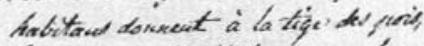

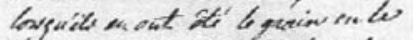

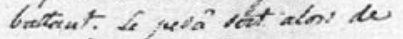

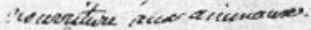

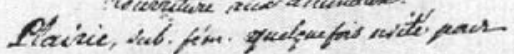
Pine, presta:

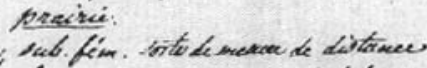

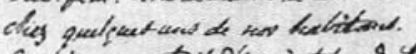
Ambier y.a til gici i tel cushith

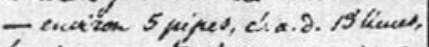

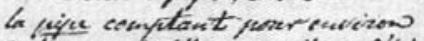

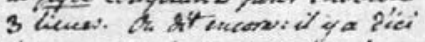

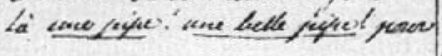

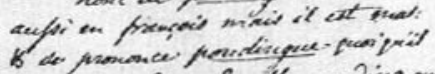

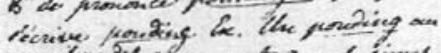

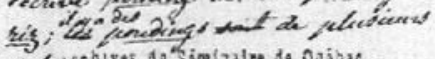

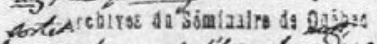

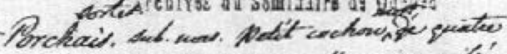

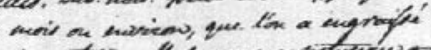

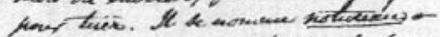

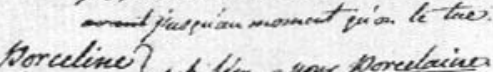

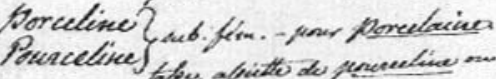

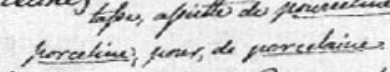

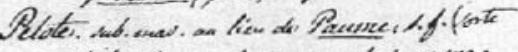

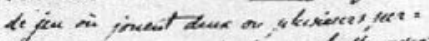

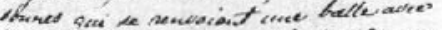

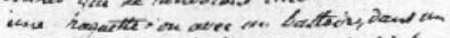

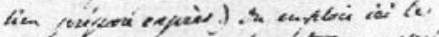

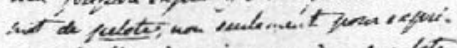

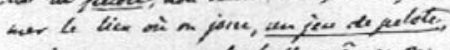

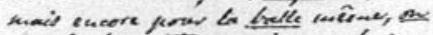

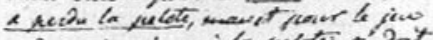

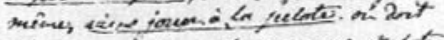

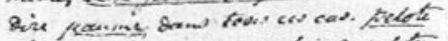

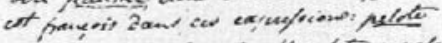

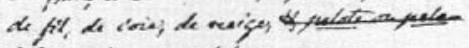
tamelarines me pelations.

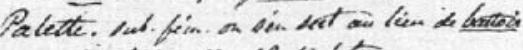

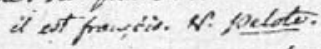

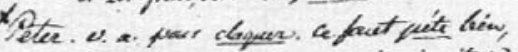

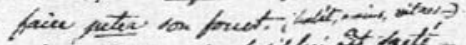

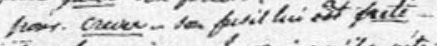

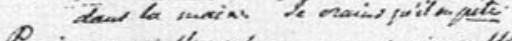

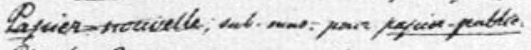

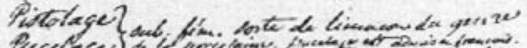

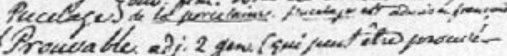

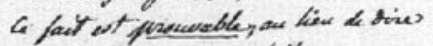

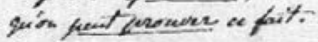

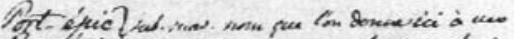

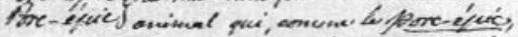

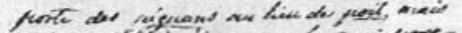

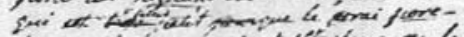

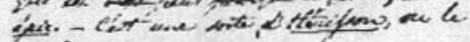
candese, on unvow

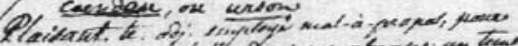

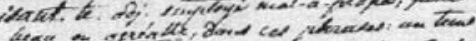

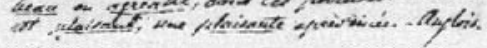

Page du manuscrit 1 de la Néologie canadienne de Jacques Viger. Manuscrit conservé au Musée de la civilisation, fonds d'archives du Séminaire de Québec. 


\section{ÉTUDE LINGUISTIQUE}


This page intentionally left blank 


\section{ÉTUDE LINGUISTIQUE}

Cette partie de l'ouvrage est consacrée à l'étude historique des faits linguistiques (phonétiques, morphologiques, syntaxiques et lexicaux) qui figurent dans la Néologie canadienne de Jacques Viger. Le Französisches Etymologisches Wörterbuch (FEW) nous a généralement permis, tout au long de nos recherches, de trouver l'étymon des mots, la datation de leurs différents sens, ainsi que leur distribution dans les parlers français. Les glossaires dialectaux ainsi que les ouvrages récents sur les parlers régionaux (de France, de Belgique et de Suisse, notamment) nous ont apporté un complément notable d'informations dans cette recherche des origines. Il faut ajouter à cette documentation les nombreux dictionnaires et ouvrages qui traitent de la langue française et qui couvrent une période qui s'étend du $X{ }^{e}$ siècle à nos jours (v. bibliogr.).

Nous avons consulté, en outre, les ouvrages qui traitent des autres variétés de français, notamment du français de l'Acadie, de l'Ontario et des États-Unis; ces études permettent souvent de découvrir le chaînon manquant dans l'étude historique du français québécois. Tout aussi importante est la comparaison avec les créoles français, en particulier avec celui de la Réunion.

En outre, il nous a paru intéressant de suivre jusqu'à nos jours l'évolution en français québécois des faits linguistiques étudiés. Nous avons consulté systématiquement les publications qui sont le fruit d'enquêtes menées dans les années 1970, soit Le parler rural de l'île-aux-Grues de M. Massicotte, Le parler populaire du Québec et de ses régions voisines. Atlas linguistique de l'Est du Canada (PPQ) de G. Dulong et G. Bergeron, Les parlers français de Charlevoix, du Saguenay, du Lac-Saint-Jean et de la Côte-Nord de Th. Lavoie, ainsi que Les parlers français d'Acadie de G. Massignon, basé sur des enquêtes faites en 1946.

Par ailleurs, le Dictionnaire québécois d'aujourd'bui (DQA) nous a fourni des indications sur le sens et l'usage actuels de bon nombre de termes et d'expressions retenus par Viger au début du XIX ${ }^{\mathrm{c}}$ siècle.

Enfin, on trouvera ci-dessous, la localisation des parlers d'oil et francoprovençaux : 


\section{OÏL}

Nord : Picardie, Artois, Flandres, Belgique wallonne.

Nord-Ouest : Normandie, Bretagne romane, Maine, Anjou, Perche.

Ouest : Poitou, Aunis, Saintonge, Angoumois.

Centre : Orléanais, Touraine, Berry, Nivernais, Bourbonnais.

Centre-Nord : Île-de-France, Champagne.

Centre-Est : Bourgogne.

Nord-Est : Lorraine, Alsace.

Est : nord de la Franche-Comté.

\section{FRANCO-PROVENÇAL}

Sud de la Franche-Comté, Suisse romande, Val d'Aoste, Lyonnais, Savoie, nord du Dauphiné. 


\section{ASPECTS PHONÉTIQUES}

\section{VOCALISME}

\section{[ $\mathrm{p}$ ] postérieur sombre à la place de [a] antérieur}

abât (Ms. 1 Cahier A; Ms. 2). Littré laisse le choix de deux prononciations pour ce mot, soit $a b a$ ou $a b a \hat{a}$. Graphie et prononciation attestées, en France, dans les parlers de l'Ouest (RézOuest). Données dans Glossaire.

âbre (Ms. 1 Cahier A). La prononciation âbre est largement attestée dans les parlers d'oïl (FEW 25, 88a sous arbor). Le [D] postérieur sombre constitue l'une des caractéristiques de la prononciation du français québécois ancien et actuel; il est généralisé dans plusieurs positions (JunPron 52-53; GendrRur 183-184; GendrTend 79-86). Cependant, cette prononciation est de plus en plus sentie comme vieillie de nos jours, et certaines catégories de mots échappent à cette prononciation (DumPron 147-149). Consignée dans Glossaire (sous âbe et âbre). Relevée également en Acadie (Massignon 274).

brâsser (Ms. 1 Cahier B; Ms. 2). Graphie et prononciation relevées en Anjou notamment (VerrAnj). Consignées dans Dionne et Glossaire.

câdre (Ms. 2). Graphie et prononciation attestées en Anjou et en Touraine (VerrAnj; Dav'Tour sous câde). Données dans Glossaire.

ébrâiller (Ms. 1 Cahier A; Ms. 2). Cette prononciation a été relevée notamment en angevin et en wallon (FEW 1, 480a sous braca; VerrAnj). Graphie et prononciation qui sont attestées dans Glossaire sous la forme adjectivale ébrâillé, ée.

éjârer (s' ) (Ms. 1 Cahier B). Graphie relevée par Dionne. Glossaire donne cette prononciation sous éjarrer.

gâ (Ms. 1 Cahier A; Ms. 2). Prononciation largement répandue en langue d'oul et en franco-provençal (FEW 17, 617b sous *wrakkjo). Le [p] postérieur sombre est particulièrement fréquent, en français québécois, en finale absolue accentuée (GendrRur 183).

gouliâ (Ms. 1 Cahier A ; Ms. 2). Glossaire donne cette prononciation sous gouliat. Recueillie lors d'enquêtes orales dans les années 1970 (PPQ 250A).

pesâ (Ms. 1 Cahier A). Graphie et prononciation qui sont connues notamment des parlers vendômois et saintongeais (FEW 8, 608a sous pisum; MussSaint). Glossaire a consigné cette prononciation sous pesat. Recueillie lors d'enquêtes orales dans les années 1970 (PPQ 899). 


\section{[ar] pour [cr]}

arse (Ms. 1 Cahier B; Ms. 2). Arse est la forme qu'a prise, en français québécois, le mot normand airse (MoisyNorm). En français, le remplacement de $[\varepsilon r]$ par [ar] est un fait d'origine populaire qui se fait surtout sentir en moyen français; il est encore largement attesté dans le parler populaire de Paris au XVII s. Il a été relevé également dans les parlers de l'Ouest et du Centre. Ce trait phonétique, très largement attesté au Québec, a été relevé dans les documents d'archives dès le $\mathrm{XVII}^{\mathrm{e}}$ s. (JunPron 39-42). Arse est consigné dans Dunn et Glossaire; Clapin et Dionne donnent, en outre, la graphie arce. Prononciation recueillie dans les années 1970 (Lavoie 1914).

sarpidon (Ms. 1 Cahier A; Ms. 2). Le mot n’a été relevé dans les parlers français que sous la forme serpida. Cependant, la permutation de [cr] en [ar] est largement attestée, dans les parlers gallo-romans, dans des mots de la même famille, comme serpent par exemple (FEW 11, 519b sous serpens). Prononciation relevée dans Glossaire qui donne, outre sarpidon, sarpida et serpida.

\section{[e] à la place de [a]}

gérémium (Ms. 1 Cahier A). Prononciation due à l'assimilation; peut-être sous l'influence de la prononciation anglaise, qui n'est pas à écarter dans le cas présent. Consignée dans Dionne; Glossaire la donne sous gérémiôme et gérénium. Recueillie lors d'enquêtes orales en 1970 (PPQ 1688x; Lavoie 343).

\section{[e] à la place de $[\varepsilon]$}

[nége] (Ms. 2 sous abât, bordée et giboulée). «Dans le parler populaire, la voyelle $e$, en syllabe accentuée, fermée par les consonnes $r, z, j$, se prononce ém (GendrRur 187-188). Prononciation enregistrée dans Glossaire; encore bien vivante.

\section{[e] à la place de [ə]}

[pésant] (Ms. 1 Cahier A sous berline). Cette prononciation résulte de l'hésitation qui a eu cours entre [ə] et [e] du XVI ${ }^{\mathrm{e}}$ au XVIII ${ }^{\mathrm{e}} \mathrm{s}$. (JunPron 114-115). Figure dans Clapin (pésant «cauchemar») et Glossaire. Recueillie dans les années 1970 (PPQ1163). 


\section{[ع] à la place de [we]}

braye (Ms. 1 Cahier B). La graphie braye (braie dans certains parlers) pour broie est consignée dans les dictionnaires des $\mathrm{XVII}^{\mathrm{e}}$ et XVIII ${ }^{\mathrm{e}} \mathrm{s}$. (Cotgrave; Encyclopédie; Trévoux 1771). La prononciation braye est attestée dans les parlers du Nord-Ouest, de l'Ouest et du Centre (FEW 15/1, 267a-b sous *brekan). Relevée dès 1715 dans les documents de la Nouvelle-France (JunPron 57). Prononciation consignée dans Glossaire. Relevée lors d'enquêtes orales au Québec (PPQ2310; Lavoie 1611) et en Acadie (Massignon 1010).

brayer (Ms. 1 Cahier B). La prononciation brayer est attestée au XVe ${ }^{\mathrm{e}} \mathrm{s}$. Largement relevée dans les parlers du Nord-Ouest, de l'Ouest et du Centre ainsi qu'en franco-provençal (FEW 15/1, 267a sous *brekan). Consignée dans Glossaire. Recueillie lors d'enquêtes orales au Québec (PPQ 2310; Lavoie 1606) et en Acadie (Massignon 1011).

fréte, fréde (Ms. 1 Cahier A). Survivance de l'ancienne prononciation en [ع] largement attestée dans le domaine d'oil (FEW 3, 797b sous frigidus). Le substantif et l'adjectif avaient respectivement les formes freit et freide au $\mathrm{XI}^{\mathrm{e}} \mathrm{s}$. Froit et froide datent du $\mathrm{XII}^{\mathrm{e}}$ s. et froid du $\mathrm{XIV}^{\mathrm{e}} \mathrm{s}$. (ReyHist sous froid, froide). Vaugelas préconise encore la prononciation fraid, tandis que Ménage considère qu'elle appartient au discours familier (RossPron 197, n. 12). Fret est attesté dès la fin du XVII s. dans des documents de la Nouvelle-France (JunPron 57). Fret et frette sont encore usités en français québécois (DQA qui ajoute sous froid ou fret: :Le mot fret s'emploie aussi et familièrement dans la plupart des sens et expressions»). Prononciation relevée en Acadie (Massignon 1188) et en Louisiane (ReadLouis).

La prononciation fréde a été relevée notamment dans les parlers du Nord-Ouest, de l'Ouest et du Centre (MénAnj sous fraide; MartVend sous fred; Massignon 1188). Elle semble disparue en français québécois.

\section{[Er] pour [a]}

berlancille, berlanciller (Ms. 1 Cahier A; Ms. 2). Le remplacement de [a] par [er] dans berlancille et berlanciller est connu en Orléanais. Cette prononciation est largement attestée, en outre, dans des mots de la même famille, notamment dans les parlers du Centre. Cf. berlancer en Touraine et en Anjou, berlançoire en Touraine; cf. également berloncer en wallon (FEW 1, 362b-363a sous bilanx). Peut-être faut-il y voir une analogie avec bercer. Prononciation consignée dans Clapin et Glossaire. 


\section{Alternance [i] - [e]}

lichefrite (Ms. 1 Cahier A; Ms. 2). La graphie lichefrite pour lèchefrite a figuré dans les dictionnaires français du XVI ${ }^{\mathrm{e}}$ au $\mathrm{XIX}^{\mathrm{e}}$ s.; Trévoux 1704-1771 la relève avec la mention "provincial»; connue notamment en picard, en normand, en poitevin, en wallon et en Suisse romande (FEW 16, 461b sous lekkon; HumbGen). Attestée dès 1685 dans des documents de la Nouvelle-France (JunPron 243-244). Consignée dans Glossaire. La prononciation lichefrite a été recueillie lors d'enquêtes orales dans les années 1970 (Lavoie 2140).

licher (Ms. 1 Cahier A; Ms. 2). Prononciation du français familier, attestée dès le $\mathrm{XII}^{\mathrm{e}} \mathrm{s}$. et probablement due à l'influence de lisser. Usitée en français populaire et dans la plupart des parlers gallo-romans (FEW 16, 459a-b sous lekkon; Bauche; $\mathrm{BW}^{5}$ et ReyHist sous lécher). Consignée dans Potier (HalPot 146). Encore usuelle en français québécois (DQA ajoute sous lécher : «Le mot licher s'emploie aussi et familièrement dans la plupart des sens et expressions"). Relevée en Acadie (Massignon 1482) et en Louisiane (ReadLouis).

\section{[i] à la place de [ع]}

arignée (Ms. 1 Cahier A). Arignée figure sous la forme arigner dans Palsgrave 1530 (cité dans TLF). Elle est consignée dans les dictionnaires des $\mathrm{XVII}^{\mathrm{e}}$ et $\mathrm{XVIII}^{\mathrm{e}}$ s. comme prononciation à corriger (Ménage; Richelet 1728 cité dans FEW). Après avoir énuméré sous araignée quelques formes dont arignée, Féraud 1787 ajoute : «Il n’y a de bon qu'araignée.» Attestée dans les parlers du Nord-Ouest, du Centre ainsi qu'en Bourgogne (FEW 25, 79a sous araneus). Consignée dans Glossaire. Relevée en Acadie (Massignon 454).

porceline, pourceline (Ms. 1 Cahier A). La forme porceline est attestée au $\mathrm{XVI}^{\mathrm{e}}$ s. Elle figure avec la mention "populaire" notamment chez Richelet 1680; Desgranges 1821 relève la forme pourceline et lui donne la mention "paysan» (cité dans FEW). Cette prononciation a été relevée en Picardie, en Normandie, dans le Maine, en Champagne et en wallon (FEW 9,187a sous porcellus). Porceline est bien attestée dans les documents du XVIII ${ }^{e}$ s. (JunPron 97). Consignée dans Glossaire (sous porceline et pourceline).

\section{[jo] pour [o]}

sieau (Ms. 1 Cahier A; Ms. 2). «La prononciation iau, née de la fermeture du premier élément de l'ancienne triphtongue eau, a vécu en français populaire de la fin du XIII ${ }^{e}$ siècle au commencement du XVII ${ }^{e}$ " 
(JunPron 70). Elle est connue des parlers du Nord, du Nord-Ouest, de l'Ouest et du Centre ainsi qu'en Suisse romande (FEW 11, 661a sous * sitellus). Très largement attestée, dès 1686, dans les documents de la Nouvelle-France (JunPron 69-71). Consignée dans Glossaire (sous siau). Recueillie lors d'enquêtes orales dans les années 1970 et très répandue (Massicotte V-189; PPQ 36 notamment; Lavoie 610, 611, 1288). Figure avec la graphie siau et la mention «anciennement ou familier» dans DQA (sous seau). Connue également en Acadie (Massignon 1181) et en franco-américain du Missouri (DorrSteGen).

\section{[o] à la place de [a]}

boyard (Ms. 1 Cahier A). Boyard qui est un apport des parlers de l'Ouest (FEW 1, 207b sous bajulus), s'est imposé en Nouvelle-France au lieu de bayard qui est la forme du français général. Il est relevé dès 1672 chez Nicolas Denys avec la graphie boyar (cité dans Massignon 609). Boyard et boyart figurent dans les documents québécois du XVIII $\mathrm{s}$. (JunPron 58). Potier consigne boyard et bayard (HalPot 75, 146). Relevé sous boyart avec la prononciation [bwejar] dans Glossaire. Recueilli sporadiquement dans les années 1970 avec la prononciation [bojar], le mot étant surtout prononcé [bwejar] (PPQ 2310; Lavoie 928). Connu également en Acadie (Massignon 609) et en francoontarien (LemVieux 25, 276).

otocas (Ms. 1 Cahier A). La prononciation otoca, plus rare qu'atoca et ataca, est due à une assimilation. Elle est attestée dès 1757 chez Bougainville : "Un petit fruit sauvage appelé l'otoka» (JunLex 93). Consignée dans Clapin (app. sous atoca). Recueillie sporadiquement dans les années 1970 lors d'enquêtes orales (PPQ 1655).

\section{[u] à la place de [o]}

couronel (Ms. 1 Cahier A). Sous la forme couronnel, le mot est attesté en français général du XVI ${ }^{\mathrm{e}}$ s. jusqu'au début du XVII ${ }^{\mathrm{e}}$ s. Prononciation également observée dans les parlers du Nord, en Saintonge et en langue d'oc (FEW 2/2, 934b sous columna; TLF sous colonel). La tendance à transformer [o] en [u] a eu cours du XIII ${ }^{\mathrm{e}} \mathrm{s}$. jusqu'au XVI ${ }^{\mathrm{e}} \mathrm{s}$. en français populaire. Ce trait phonétique est bien attesté dans les documents de la Nouvelle-France (JunPron 15).

pourceline (Ms. 1 Cahier A). La prononciation en [u] dans pourceline, attestée dès le XIII ${ }^{e} \mathrm{~s}$, , est plus ancienne que celle en [o] de porcelaine qui date du début du XVI ${ }^{\mathrm{e}} \mathrm{s}$.; Desgranges 1821 consigne pourceline avec la mention "paysan" (cité dans FEW). Les prononciations pourcelaine et pourceline ont été relevées en Normandie, dans le Maine, en 
Champagne et en Franche-Comté (FEW 9, 187a sous porcellus). Pourcelaine figure dès 1732 dans des documents de la Nouvelle-France (JunPron 19). Prononciation consignée dans Glossaire (sous pourcelaine et pourceline).

\section{[u] à la place de [y]}

brouscailler (Ms. 1 Cahier B). Variante phonétique du verbe brusquailler; ce dernier est attesté en normand et en saintongeais (FEW 1, 575b sous bruscum; MussSaint). Cf., en Anjou, la prononciation en [u] attestée dans un verbe de la même famille, se brousquer (FEW $i b$.). L'alternance de $[\mathrm{u}]$ et $[\mathrm{y}]$ était fréquente anciennement en français (RossPron 213). Cf. également dans Potier enfouitt pour [ãfyi] (HalPot 148). Consignée dans Glossaire (sous brousquâiller) qui enregistre également brusquâiller.

\section{[we] à la place de [wa]}

gouette (Ms. 1 Cahier A). Le terme est attesté en français sous la forme gouetre de 1561 au début du XIX ${ }^{\mathrm{e}}$ s. (FEW 4, 353b sous guttur). Bescherelle 1858 consigne les graphies goître et goètre. Relevé en Normandie sous la forme goite et en Touraine sous gouête (FEW ib.; DavTour). Prononciation consignée dans Glossaire (sous goite). Largement attestée lors d'enquêtes orales au Québec (PPQ 2214; Lavoie 2491) et en Acadie (Massignon 1602).

ouète (Ms. 1 Cahier A). La graphie ouète est consignée dans Académie jusqu'en 1878 (TLF sous ouate); Bescherelle 1858, qui critique cette prononciation, fait le commentaire suivant : «L'Académie dit que l'on doit prononcer ouète, ouèter. Nous pensons cependant que cette prononciation, bien qu'assez usitée, n'en est pas moins vicieuse, et qu'elle doit être proscrite.» Donnée avec la mention «vieilli» dans DG. Attestée dès 1732 dans les documents d'archives québécois (JunPron 62). Recueillie dans les années 1970 (PPQ138x).

Quant à la règle qu'évoque Viger au sujet de l'élision ou non de l'article ou de la préposition devant ouate, on peut dire que les deux solutions sont admises, mais que pour des raisons d'euphonie l'élision semble l'emporter (TLF sous ouate).

\section{[o] à la place de [wa]}

épotraillé (Ms. 1 Cahier B). Le remplacement de [wa] par [o] a été observé dans des mots de la même famille, tels que dépotraille, attesté 
en Champagne notamment; potrail a été relevé en Picardie, en Champagne et dans les parlers du Centre (FEW 8, 109a sous pectoralis); cf. également potrine dans les parlers du Nord, du Nord-Ouest et de l'Ouest (FEW 8, 110a sous "pectorinus). Relevé dans Glossaire (sous épotrâillé, ée). Ce trait phonétique est bien attesté en français québécois (Lavoie 1476 sous poitrail et 2363 sous poitrine).

\section{CONSONANTISME}

\section{LES CONSONNES FINALES}

\section{Amuissement de [l] après consonne labiale}

trèfe (Ms. 1 Cahier A). Prononciation relevée dès le XVe s. et largement connue de tout le domaine d'oil ainsi qu'en Suisse romande (FEW 13/2, 293b- 294a sous triphyllon; RollFlore IV, 141-142). Pour une étude détaillée de l'amuïssement du [1] en français québécois, v. JunPron 173-174. Consignée dans Glossaire. Recueillie dans les années 1970 (PPQ790; Lavoie 1117).

\section{Amuïssement de [r] après consonne $+[\mathrm{r} ə]$}

gouette (Ms. 1 Cahier $\mathrm{A})$. L'amuïssement de $[\mathrm{r}(\partial)]$ après une consonne a eu cours dans le parler populaire de l'île-de-France au XVII ${ }^{\mathrm{e}}$ s.; il a été observé dans ce mot notamment en Normandie (goîte) et en Touraine (gouête) (FEW 4, 353b sous guttur; DavTour). Largement attesté en français québécois ancien et actuel (JunPron 205-208). Donné dans Clapin (sous goite), Dionne (sous goitte) et Glossaire (sous goite). Relevé lors d'enquêtes orales au Québec (PPQ 2214; Lavoie 2491) et en Acadie (Massignon 1602).

\section{Amuïssement de [k]}

bourasse (Ms. 1 Cahier B). De bourrasque francisé en -ache et -asse au $\mathrm{XVI}^{\mathrm{e}}$ s. (BW ${ }^{5}$ sous bourrasque; GodCompl; Huguet). Prononciation qui est connue des parlers du Centre et que FEW relève non pas sous l'étymon boreas $(1,441 \mathrm{~b})$, mais sous celui de burra $(1,638 \mathrm{~b})$. Bour(r)asse est probablement à rapprocher de prononciations telles que séminarisse, batisse, cataplas qui attestent l'amuïssement de certaines consonnes finales (JunPron 207-208). Consignée dans Glossaire (sous bourrasse). 


\section{Maintien de [s] final}

deusse (Ms. 1 Cahier A). Les consonnes finales se sont prononcées longtemps en français. Au début du $\mathrm{XVI}^{\mathrm{e}}$ s., elles étaient encore prononcées devant une pause. Cette tendance s'est maintenue, en français moderne, dans des mots tels que dix, six, neuf, plus, tous; deux et trois faisaient partie de ce groupe de mots mais cette prononciation n'est plus acceptée aujourd'hui (Nyrop I, $\mathrm{n}^{\circ} 315$ ). Attestée en Normandie, en Anjou et en Touraine; en Bourgogne, elle désigne le féminin (FEW 3, 181a sous duo; VerrAnj); donnée comme variante populaire et argotique dans TLF (sous deux). Consignée dans Glossaire. En Acadie, deusse désigne le féminin chez quelques informateurs et les deux genres chez d'autres (Massignon 1431).

troisse (Ms. 1 Cahier A). Même explication historique que pour deusse. Prononciation attestée en Normandie et en Bretagne notamment (FEW 13/2, 247b sous tres). Consignée dans Glossaire (sous troisses).

\section{Maintien du [t] final}

fréte (Ms. 1 Cahier A). D'abord écrit freit au $\mathrm{XI}^{\mathrm{e}}$ s. et froit du XII ${ }^{\mathrm{e}}$ au $\mathrm{XIV}^{\mathrm{e}}$ s., puis froid au XIVe s. (ReyHist sous froid, froide). Le maintien $\mathrm{du}[\mathrm{t}]$ final est hérité de l'ancienne prononciation de froid qui, jusqu'au $\mathrm{XVII}^{\mathrm{e}}$ s., faisait entendre le [d] final comme un [t] (RossPron 245). Cette prononciation est connue notamment en Touraine, en Anjou, en Poitou, en Saintonge et en franco-provençal (FEW 3, 797b sous frigidus; VerrAnj sous fret'; MussSaint sous fret). Bien attestée en français québécois ancien et actuel (JunPron 188-194; GendrRur 189; DQA qui fait une double entrée : Froid, froide ou Fret, frette). Trait phonétique connu en Acadie (Massignon 1188) et dans les parlers franco-américains de la Louisiane (ReadLouis) et du Missouri (DorrSteGen).

\section{Ajout d'un [t]}

icit (Ms. 1 Cahier A). Prononciation attestée dans la région parisienne au $\mathrm{XVII}^{\mathrm{e}}$ s. chez Tallemant des Réaux, sous la graphie icyte; surtout connue des parlers du Nord-Ouest, de l'Ouest et du Centre et encore en usage, de nos jours, en Touraine notamment (FEW 4, 423b sous bic; TLF; SimTour). Le [t] non étymologique est probablement le résultat d'une lutte entre plusieurs formes de l'ancien français qui signifiaient "ainsi» et qui ont été léguées à «ici», par une influence analogique, soit issi, issin(t), issit (House 147-150; JunJum 70). Relevée en 1744 au Détroit par Potier (HalPot 151, 273). Consignée dans 
Glossaire (sous icite). Encore usuelle de nos jours au Québec (DQA ajoute sous $i c i$ : «La variante icitte est fréquente et elle appartient au registre familier»).

légerte (Ms. 1 Cahier A; Ms. 2). Relevée en wallon sous la graphie ligerte (FEW 5, 287a sous *leviarius). Cette prononciation s'est imposée au moment où le $[\mathrm{r}]$ de léger se prononçait (il se prononce encore à l'île aux Grues et en Acadie); elle s'oppose à léger comme offerte s'oppose à offert, ouverte à ouvert (Massicotte II-18; DumPron 33). Comme le fait remarquer $\mathrm{M}$. Massicotte l'influence $\mathrm{du}[\mathrm{t}]$ de légèreté n'est peutêtre pas à écarter. Consignée dans Glossaire (sous légearte et légerte). Recueillie lors d'enquêtes orales au Québec (Massicotte ib. ; PPQ 693; Lavoie 128, 129 et 145) et en Acadie (Massignon 1425).

\section{LES CONSONNES À L'INTÉRIEUR DU MOT}

\section{Amuïssement de [r] implosif}

âbre (Ms. 1 Cahier A). L'amuïssement du [r] implosif dans ce mot remonte au XII ${ }^{e}$ s. En usage encore au XVII ${ }^{\text {s. }}$. (RossPron 297), mais condamné par Vaugelas. Anciennement, le $[r]$ disparaissait souvent quand la syllabe suivante ou précédente contenait un autre $[\mathrm{r}]$ (Nyrop I, $\mathrm{n}^{\circ}$ 513). Il est attesté partout dans le domaine d'oil ainsi qu'en Suisse romande (FEW 25, 88a sous arbor; HumbGen). Consigné dans Glossaire (sous âbe et âbre). Recueilli lors d'enquêtes orales au Québec (PPQ1257; Lavoie 352) et en Acadie (Massignon 274).

notureau (Ms. 1 Cahier A). Les graphies de ce dialectalisme de l'Ouest sont nombreuses et comportent généralement un [r]. L'amuïssement du [r] a été relevé en Normandie (FEW 7,253b sous nutritura). Bien attesté dans les documents d'archives du XVII ${ }^{\mathrm{e}}$ s. (JunPron 163, 234).

\section{Hésitation entre [r] et [1]}

couronel (Ms. 1 Cahier A). Prononciation attestée en français de 1542 jusqu'au début du XVII ${ }^{e}$ s.; elle a été relevée dans le parler de Paris (Desgranges 1821, cité dans FEW), dans les parlers de l'Est, en wallon et en langue d'oc (FEW 2/2, 934b sous columna; TLF sous colonel). La substitution réciproque du [r] et du [1] est un trait phonétique très répandu dans le français des $\mathrm{XVI}^{\mathrm{e}}$ et $\mathrm{XVII}^{\mathrm{e}}$ s.; également bien attestée dans les documents d'archives au Québec (JunPron 161-162). Consignée dans Glossaire (sous coronet). 
plairie (Ms. 1 Cahier A). Prononciation relevée au XIII ${ }^{\mathrm{e}}$ s. sous la graphie plaerie (FEW 9, 334a sous pratum). L'hésitation entre [r] et [1], qui a eu cours aux $\mathrm{XVI}^{\mathrm{e}}$ et $\mathrm{XVII}{ }^{\mathrm{e}}$ s., en France, est largement attestée dans les documents québécois (JunPron 159-162). La prononciation plairie est consignée dans Glossaire et a été recueillie lors d'enquêtes orales au Québec (PPQ 519; Lavoie 1114) et en Acadic (Massignon 689). Connue également en Louisiane (DitchyLouis).

\section{[1] et [r] parasites}

flamblant (Ms. 1 Cahier B; Ms. 2). La présence du l étymologique est attestée du XII ${ }^{\mathrm{e}}$ au XVe $\mathrm{X}$. dans le substantif flamble (du lat. flammula); il s'est effacé par dissimilation (Nyrop I, $\mathrm{n}^{\circ} 513$; Bourciez n ${ }^{\circ} 185$, rem. II; ReyHist sous flamber; FEW 3,602a sous flammula). Nisard relève flamblé encore au $\mathrm{XIX}^{\mathrm{e}}$ s. dans le parler populaire de Paris (NisÉt 269).

cartron (Ms. 1 Cahier A). Prononciation relevée en saintongeais et dans les parlers de l'Est (FEW 2/1,627b sous charta). L'ajout d'un [r] parasite après une consonne intérieure par suite d'une assimilation progressive a donné entre autres les formes perdrix, dartre, tertre, et anciennement jardrin (Bourciez n ${ }^{\circ} 178$, rem. III). Cartron figure dans Glossaire.

chardron (Ms. 1 Cahier A). Prononciation bien attestée dans le domaine d'oil (FEW 2/1, 368a sous carduus). Même explication historique que pour cartron. Relevée dès le XVIII ${ }^{\mathrm{e}} \mathrm{s}$. dans les documents québécois (JunPron 216). Consignée dans Glossaire. Recueillie lors d'enquêtes orales au Québec (PPQ1026; Lavoie 319) et en Acadie (Massignon 228).

\section{[3] à la place de [z]}

georges (faire ses $\sim$ ) (Ms. 1 Cahier A; Ms. 2). Faire ses [3or3ə] pour faire ses [zorza] illustre la tendance, d'origine normanno-picarde, à substituer [3] à [z]. Les documents d'archives québécois en donnent quelques exemples, dont deux jomme [sic] pour deux ( $\left.z^{\prime}\right)$ hommes relevé au XVIII s. (JunPron 140-141). Il peut s'agir également ici d'une étymologie populaire.

\section{[m] à la place de [n]}

gérémium (Ms. 1 Cahier A). Le remplacement de [n] par [m] figure, sous la forme géromium, dans Platt 1835; relevé dans les parlers du Nord- 
Ouest, de l'Ouest et du Centre (FEW 4, 118a sous geranion). Consigné dans Glossaire (sous gérémiôme) et recueilli encore dans les années 1970 (PPQ1688x; Lavoie 343).

\section{Ajout d'un [t]}

castonade (Ms. 1 Cahier A). Attestée dès le $\mathrm{XVI}^{\mathrm{e}}$ s., la prononciation castonade est très largement attestée dans les parlers d'oil ainsi qu'en Suisse romande (FEW 2/2,1430b sous quassare; HumbGen). Admise dans Académie 1718. Proscrite dans les dictionnaires des XVIII ${ }^{\mathrm{e}}$ et $\mathrm{XIX}^{\mathrm{e}}$ s. (TLF sous cassonade). La forme castonade serait due à l'influence de bastonnade (Nyrop I, $\mathrm{n}^{\circ}$ 118). Les documents de la Nouvelle-France attestent cette prononciation dès 1676 (JunPron 246). Consignée dans Glossaire, et encore recueillie dans les années 1970 (Lavoie 2205).

thétière (Ms. 1 Cahier A). Prononciation consignée dans Trévoux 1732 qui ajoute dans l'édition de 1771 : «L'usage est pour théière." Relevée dans les parlers du Nord, en wallon et en franco-provençal (FEW 20, 112 a sous teb). La prononciation thétière serait due à l'influence de cafetière (BW ${ }^{5}$ sous the $)$. Les documents d'archives attestent cette graphie dès 1710 en Nouvelle-France (JunPron 245-246). Glossaire consigne cette prononciation (sous thétiére); encore largement répandue dans les années 1970 (Lavoie 2147).

\section{Métathèse [rə] devient [er]}

berdas, berdasser (Ms. 1 Cahier B sous bredas, bredasser). Viger ayant hésité entre les deux formes de cette famille de mots dans son manuscrit (v. notes 52 et 54 du ms. 1), il convient ici d'en faire mention. La prononciation berdas, berdasser pour bredas, bredasser est bien attestée dans les parlers du Nord-Ouest et de l'Ouest (FEW 1,540b sous brittus; MinPoit). Consignée dans Glossaire (sous berda, berdasser). En français québécois actuel, le nom et le verbe sont surtout usités sous les formes barda et bardasser (DQA). Connue en Acadie (Massignon 1219 berdasser, beurdasser) et en Louisiane (ReadLouis beurdasser).

berloque (Ms. 1 Cahier A). Berloque pour breloque est bien attesté dans le domaine d'oil (FEW 8, 567b-568a sous *pir-). Donné dans Dunn, Clapin, Dionne et Glossaire. Recueilli en Acadie (PoirAcad).

ferlasser (Ms. 1 Cahier B). Prononciation relevée dans les parlers du Centre et de l'Ouest (FEW 23, 202b sous bruire; MinPhon 161). P. Rézeau qui relève le mot sous les formes ferlasser et frelasser suggère de le rattacher à FEW 3, 815a-b sous frl-(RézVoc). Consignée dans 
Dunn et Clapin (sous ferdasser, ferlasser), Dionne (sous ferdasser) et Glossaire (sous ferdasser et ferlasser).

\section{Amuïssement du préfixe [rə]}

brousse-poil (à $\sim$ ) (Ms. 1 Cahier A). L'amuïssement du préfixe ne semble pas avoir été relevé ailleurs qu'en français canadien. Consigné dans Dionne et Glossaire. Recueilli en Acadie (Massignon 992).

\section{SEMI-CONSONNE}

\section{[j] à la finale}

braye (Ms. 1 Cahier B). La graphie braye pour broie est attestée en français depuis Cotgrave 1611; relevée en Normandie, dans le Berry, en Anjou, en Poitou et en wallon (FEW 15/1, 267a-b sous ${ }^{*} b r e k a n$ ). Le [j] s'explique, selon M. Juneau, par une analogie des formes de l'indicatif imparfait, de l'infinitif, etc., du verbe broyer (JunPron 205). Glossaire consigne cette prononciation sous braye et breille. Elle a été recueillie lors d'enquêtes orales au Québec (PPQ 2310; Lavoie 1611) et en Acadie (Massignon 1010).

\section{AGGLUTINATION}

avisse (Ms. 1 Cahier A). Cette prononciation a eu cours au XVIII et au $\mathrm{XIX}^{\mathrm{e}}$ s. Donnée avec la mention "vieux" à la fin du XIX ${ }^{\mathrm{e}}$ s.; Bescherelle 1858 écrit : «Vieux mot qui désignait une pièce de fer ou de cuivre, etc., qui était à vis.» Attestée dans les parlers du Nord, du Nord-Ouest, de l'Ouest ainsi qu'en langue d'oc (FEW 14, 558b sous vitis). Résulte soit d'une mauvaise coupure de la dans la vis, soit d'une soudure de la préposition à dans à vis (JunPron 217; MussSaint). Consignée dans Glossaire.

écopeau (Ms. 1 Cahier A). La prononciation de ce mot provient de l'agglutination de l'article; attestée au XVI ${ }^{\mathrm{e}}$ s. Elle a été observée dans de nombreux parlers d'oïl, tels que ceux du Nord-Ouest, de l'Ouest, du Centre, du Nord-Est, ainsi qu'en franco-provençal (FEW 2/2, 1594a sous cuspis). Recueillie au Québec dans les années 1970 (Massicotte V-58; PPQ 129; Lavoie 594). Également connue en Acadie (Massignon 298) et dans les parlers franco-américains de la Louisiane (ReadLouis; DitchyLouis) et du Missouri (DorrSteGen). 
lévier (Ms. 1 Cahier A). Prononciation qui relève du français populaire (Bauche). «Le peuple dit par corruption léviem (Bescherelle 1858 sous évier). La forme lévier (souvent lavier) est connue notamment en Picardie, dans les parlers du Nord-Ouest, en Champagne, en Bourgogne et en Suisse romande (FEW 25, 70b-71a sous aquarius). Recueillie dans les années 1970 (PPQ 100). S'entend encore de nos jours.

\section{ÉTYMOLOGIE POPULAIRE}

corporal (Ms. 1 Cahier A). Forme altérée de caporal sous l'influence de corps; attestée de 1562 à Trévoux 1771. Relevée en normand, en poitevin, en wallon et en franco-provençal (BW ${ }^{5}$ sous caporal; FEW 2/1, 344a sous caput). Consignée dans Clapin, Dionne et Glossaire. La présence du mot anglais corporal a pu contribuer au maintien de cette forme ancienne pendant un certain temps en français québécois. Cette forme semble sortie d'usage aujourd'hui.

mondaine (Ms. 1 Cahier A; Ms. 2). La forme mondaine pour mondé (dans orge mondaine) a été relevée par Dionne. Recueillie à quelques points d'enquête dans les années 1970 (PPQ768s).

port-épic (Ms. 1 Cahier A). Le mot est attesté en français populaire en 1808 et dans Platt 1835, sous les formes porte-pic et porte-pique. Relevé en Anjou (portépic), dans le Maine et dans le Limousin (FEW 9, 191a sous porcus). Attesté en Nouvelle-France dès 1648 sous la forme porte epic (Massignon 388). Recueilli lors d'enquêtes orales au Québec (PPQ 1595; Lavoie 674) et en Acadie (Massignon ib.). 


\section{ASPECTS MORPHOLOGIQUES ET SYNTAXIQUES}

\section{LE NOM ET L'ADJECTIF}

\section{NOMBRE}

\section{Pluriel en -als}

confessionnals (Ms. 1 Cahier B). Probablement par analogie avec un certain nombre de noms courants en -al qui ont leur pluriel en -s tels que bal, carnaval, festival, récital, etc. (Grevisse $\mathrm{n}^{\circ} 278$ ).

fanals (Ms. 1 Cahier B). Dupré précise que le pluriel est en -aux : «Aucune question ne se pose au sujet du pluriel de ce nom : fanaux.» Le pluriel en -als est attesté dans des documents québécois des XVIII et $\mathrm{XIX}^{\mathrm{e}}$ s. : deux fanal; 2 vitre a 2 fanale (TraLiQ 1, 93). Recueilli sporadiquement lors d'enquêtes orales au Québec (Lavoie 2042, 2043) et en Acadie (Massignon 1217).

\section{Pluriel en -aux}

étaux (Ms. 1 Cahier A). Le pluriel régulier de étal est étaux. Cependant, la forme étals tend à remplacer aujourd'hui étaux, en raison de l'homonymie avec le pluriel de étau (Grevisse n ${ }^{\circ} 278$, rem. 3; Dupré; ReyHist sous étal).

\section{GENRE}

\section{Le féminin au lieu du masculin}

argent (Ms. 1 Cahier A). Attesté au féminin dès le XIVe s. Argent est connu au féminin dans tout le domaine gallo-roman (FEW 25, 192b sous argentum). Consigné dans Clapin, Dionne et Glossaire qui donnent les syntagmes de la belle argent, de la bonne argent qui s'entendent encore de nos jours. Recueilli lors d'enquêtes orales au Québec (PPQ 1750x) et en Acadie (Massignon 1426).

fenouil (Ms. 1 Cahier A). Sous la forme fenoille, le féminin a vécu en français, du XIII ${ }^{\mathrm{e}}$ au XVI ${ }^{\mathrm{e}}$ s.; il a été relevé notamment en picard et en saintongeais (FEW 3, 454a sous fenuculum; MussSaint). 
gouette (Ms. 1 Cahier A). Le mot (gouette : goitre) est consigné au féminin dans les dictionnaires, de Furetière 1690 à Trévoux 1732 (FEW 4, $353 \mathrm{~b}$ sous guttur). Les deux genres étaient admis au XVIII $\mathrm{s}$. Furetière 1727, qui le donne au féminin, ajoute : "Quelques-uns le font masculin.» Le genre masculin s'impose au XIX ${ }^{\mathrm{e}}$ s., et c'est la formulation inverse qui est employée, dans Bescherelle 1858, notamment: «Quelques auteurs l'on fait du féminin.» Consigné dans Clapin, Dionne et Glossaire.

incendie (Ms. 1 Cahier A). Le féminin est observé dans ce mot depuis le $\mathrm{XVII}^{\mathrm{e}}$ s.; bien attesté dans les parlers d'oïl et notamment dans ceux du Nord ainsi qu'en Suisse romande (FEW 4, 620a sous incendium; HumbGen). Encore relevé au XIX ${ }^{\mathrm{e}}$ s. par Desgranges 1821 et Platt 1835 (cités dans FEW ib.). Incendie «présentant une voyelle finale longue est parfois fait féminin dans un parler fautif. On entend dire erronément : une grosse incendie» (Dupré).

poudine (Ms. 1 Cahier A). En français général, le mot anglais pudding ou pouding pour plum-pudding, emprunté en 1678 , a pris le genre masculin (FEW 18, 98b-99a sous pudding; ReyDAngl). Dans le même sens, le mot est également du genre masculin en français québécois actuel (DQA sous pouding ou pudding). En revanche, le féminin est largement employé, dans le registre familier, pour désigner le «mets fait de pâte à gâteau déposée sur des fruits» (DQA ib.).

\section{Le masculin au lieu du féminin}

araignée (Ms. 1 Cahier A). Le masculin a été relevé en Anjou ainsi que dans les parlers du Centre (FEW 25, 78a sous araneus). Consigné dans Glossaire.

sentinelle (Ms. 1 Cahier A). Sentinelle est attesté au féminin depuis le $\mathrm{XVI}^{\mathrm{c}}$ s. Le mot a cependant été usité au masculin au XVIII ${ }^{\mathrm{e}} \mathrm{s}$; consigné au masculin dans Académie 1798-1835 (FEW 11, 471b sous sentire). Féraud 1787 fait le commentaire suivant : «Sentinelle est toujours fém[inin]. Quelques auteurs l'ont fait masculin.»

tondre (Ms. 1 Cahier A). Le masculin a été relevé notamment en Normandie et en Saintonge (FEW 17, 387a sous tundr). Consigné dans Clapin, Dionne et Glossaire. Recueilli au masculin au Québec (PPQ91x; Lavoie 2013), en Acadie (Massignon 324) ainsi que dans les parlers franco-américains de la Louisiane (ReadLouis) et du Missouri (DorrSteGen). Le mot ne figure plus dans les dictionnaires usuels. 


\section{PRÉFIXATION}

\section{En $e^{-}$}

épotraillé (Ms. 1 Cahier B). Épotraillé pour dépoitraillé a été relevé en Normandie sous la forme épétrâillé (FEW 8, 109a sous pectoralis). Figure dans Clapin (sous époitrailler) et Glossaire (sous époitrâillé, ée et épotrâillé,ée).

\section{En en-}

envarié (Ms. 1 Cahier A). La forme envarié pour avarié ne semble pas avoir été relevée ailleurs qu'en français canadien. Le remplacement de $a$ - par en-fréquent anciennement, en français québécois, dans de nombreux mots tels que envaler, envec, etc., est probablement hérité des parlers français; cf. en normand envant, envertir, envouer (RobNorm). Envarié paraît dans un document québécois de 1777 . Il peut s'agir également d'un croisement de mots; comme le fait remarquer $\mathbf{M}$. Juneau, «il y a peut-être eu contamination avec enverguer, mot de la langue maritime, tout comme avarien (JunPron 246). La forme envarié est consignée dans Glossaire (sous anvarié, ée).

\section{En in-}

inquilibre (Ms. 1 Cahier B sous équilibre). Le remplacement de é-par inest probablement un apport des parlers saintongeais où il est particulièrement présent et touche un grand nombre de mots : inpouvantable, inducation, ingal, inglise, intranger, inventail, etc. (MussSaint). Il a été relevé également dans des documents québécois du XVIII ${ }^{\text {s. dans des }}$ mots tels que incarlate, inducation (JunPron 242 et 259). Inquilibre figure dans Dionne.

\section{SUFFIXATION}

\section{En -age}

marinage (Ms. 1 Cahier A). Variante de marinade «légumes marinés». Consignée dans Glossaire (sous marinages). Recueillie au pluriel lors d'enquêtes orales au Québec (PPQ 223; Lavoie 2199) et en Acadie (Massignon 1339).

\section{En -ant}

étrivant (Ms. 1 Cahier B; Ms. 2). Étrivant a été relevé anciennement en français, comme substantif, au sens de «concurrent» (Godefroy et Huguet sous étriver). Figure dans Dionne et Glossaire. 


\section{En-ante}

brunante (Ms. 1 Cahier B). Les formes brunant, brunante sont attestées comme adjectifs au XII ${ }^{\mathrm{e}}$ s. Le mot est connu comme substantif sous la forme breunant en haut-manceau et brunant en Touraine (FEW $15 / 1,307 \mathrm{~b}$ sous ${ }^{*}$ brun; Massignon 69 ).

\section{En - au au lieu de $-a l$}

étau (Ms. 1 Cahier A) Un certain nombre de noms en -al ont refait le singulier sur le pluriel et ont pris une nouvelle forme en -au, comme par exemple animau, bestiau, chevau, journau, etc. Les formes en -au pour -al remontent au Moyen Âge. Elles sont bannies de la langue littéraire dès le XVII s., mais ont subsisté dans les parlers d'oil où elles ont été très répandues (Nyrop II, ${ }^{\infty 6} 291,299-300$ ). Pour ce qui est du mot étal, le remplacement de $-a l$ en -au a pu se produire, en outre, sous l'influence de étau (d'abord estoc «presse»). Etau pour étal a vécu en français général de 1564 à Trévoux 1771 : «Quelques-uns disent au singulier étal"; ce dernier consigne dans le même article et sous le même mot vedette les deux mots étau (étau "table» et étau "presse»). La forme étau a été relevée en Orléanais, en Bourgogne et dans les parlers du Nord-Est et de l'Est (FEW 17, 206b sous ${ }^{*}$ stal). Figure dans Dionne.

\section{En -ée}

canotée (Ms. 1 Cahier B; Ms. 2). Ce dérivé de canot n'a pas été retenu par les dictionnaires français. En Nouvelle-France, au XVIII ${ }^{\mathrm{s}}$ s., le suffixe -ée a été particulièrement productif dans la création lexicale. Potier, qui relève canotée, consigne une vingtaine de mots ainsi formés (HalPot 156-158).

\section{En-erie}

bredasserie (Ms. 1 Cahier B). La forme bredasserie a été relevée en Vendée; le mot est également attesté en Touraine sous la variante berdasserie (FEW 1, 541a sous brittus; RézVoc). Potier le relève en 1744 au Détroit (HalPot 242). Figure dans Dionne et Glossaire (sous berdasserie).

$$
\text { En -eur, -eux }
$$

cheniqueur (Ms. 1 Cahier A). Dérivé de cheniquer, ce mot ne semble pas être connu ailleurs qu'en français québécois. Figure dans Clapin (sous cheniqueur, cheniqueux), Dionne et Glossaire (sous cheniqueux). 
bicleux (Ms. 1 Cahier A; Ms. 2). Bicleux pour bicle. Le suffixe en -eux est dialectal dans ce mot; relevé en Touraine (DavTour) et dans les parlers du Centre (JaubCentre sous biclu). Donné dans Glossaire.

bourasseur (Ms. 1 Cahier B). De bourasser; bourasseur et plus généralement bourasseux (Viger lui-même avait d'abord écrit cette dernière forme : v. note 46 du ms. 1) ne semblent être connus qu'en français canadien. Clapin, Dionne et Glossaire consignent bourasseux.

\section{En -ier}

bredassier (Ms. 1 Cahier B). Bien attesté dans les parlers du Nord-Ouest, de l'Ouest et du Centre sous les formes bredassier et berdassier (FEW 1, 540b sous brittus; RézVoc). Potier le relève au Détroit en 1744 (HalPot 242). Donné dans Dionne et Glossaire sous berdassier et bredassier.

\section{En -iste}

Montréaliste (Ms. 1 Cahier B). Le suffixe en -iste a eu cours du XVII au $\mathrm{XIX}^{\mathrm{e}}$ s. : Mon-Realiste 1654 ; Montrealiste 1672 ; Montréaliste 1727 (DugGent; DFP sous montréalais). Quant à la forme actuelle Montréalais, elle n'est attestée que depuis 1859 . À la fin du XIX ${ }^{\mathrm{e}}$ s., la concurrence entre les gentilés Montréaliste et Montréalais est forte. Clapin ne relève que Montréalais; Dionne (sous montréaliste) s'interroge : «Montréalais ou Montréaliste, citoyen de Montréal. Lequel vaut mieux ? [...]. On ne dit plus maintenant que Montréalais.»

\section{En-on}

sarpidon (Ms. 1 Cahier A; Ms. 2). Variante de serpida. Le suffixe -on ne semble avoir été usuel, dans ce mot, qu'en français québécois. Figure dans Glossaire.

\section{En -oux}

graissoux, ouse (Ms. 1 Cahier A). Forme bien attestée dans les parlers du Nord-Ouest et de l'Ouest, ainsi qu'en langue d'oc (FEW 2/2, 1277a sous *crassia). Du suffixe latin -osus qui a donné -eux en français et -ou, -ouse dans certains parlers, dont les parlers poitevins où ce suffixe fut particulièrement productif (MinPhon 122-127). Forme consignée dans Clapin, Dionne et Glossaire. 


\section{LE PRONOM}

stellà (Ms. 1 Cahier A). De cestuy la «celui-là» attesté au XVe s. et largement répandu sous la forme stila; également relevé en français popu-

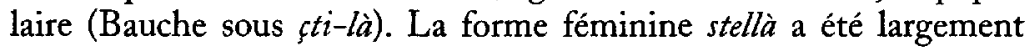
relevée en Normandie, dans le Berry, en Anjou, en Bourgogne et en wallon (FEW 4, 821a sous iste). Consignée dans Glossaire (sous stèla et stel-là). Bien attestée anciennement au Québec (GendrRur 182; JunJum 82) et en Acadie (PoirAcad qui donne stelle-là sous sti-cit).

stellci(t) (Ms. 1 Cahier A). Stellci( $t$ ), de l'ancienne forme cestui-ci «celuici» largement attestée, au masculin, sous la forme stisi tant en langue d'oil qu'en langue d'oc ainsi qu'en franco-provençal; la forme féminine stellci a été relevée notamment en Sologne (FEW 4, 820b sous iste). Consignée dans Glossaire (sous stel-ci et stel-cite).

\section{LE VERBE}

\section{PRÉFIXATION}

\section{En $a-$}

amancher (Ms. 1 Cahier A; Ms. 2). Pour emmancher. Les formes amancher et emmancher, qui datent du $\mathrm{XV}^{\mathrm{e}} \mathrm{s}$., ont coexisté en français jusqu'au XVII ${ }^{\mathrm{e}}$ s. Amancher, qui est sorti de l'usage en français général, s'est maintenu dans de nombreux parlers d'oil et en Suisse romande (FEW 6/1, 222a sous manicus). La forme amancher est encore bien vivante au Québec dans certains contextes (DQA).

\section{En $e^{-}$}

ébrâiller (Ms. 1 Cahier A; Ms. 2). Le verbe, beaucoup moins usuel que l'adjectif, a été relevé en wallon sous la forme abrâyer et sous la forme pronominale dans le Limousin (FEW 1, 480a sous braca).

Ébraillé figure au XVI ${ }^{\mathrm{e}}$ s. chez Montaigne (FEW ib.); il a été relevé en Saintonge (MussSaint). Consigné dans Dionne et Glossaire (sous ébrâillé, ée). Recueilli dans les années 1970 (Lavoie 2533).

écolter, escolter (Ms. 1 Cahier A; s'écolter Ms. 2). Sous la forme escoleter, le verbe est attesté en français des XIII' et XIV es. (FEW 2/2, 917a sous collum). L'emploi pronominal figure dans Bescherelle 1858 (sous écolletter). Au sens général de «décolleter», le verbe figure encore dans DG (sous écolleter) avec la mention «anciennement». Consigné dans Clapin, Dionne et Glossaire (sous écolleter). 
L'emploi adjectival a été observé dans les parlers du Nord-Ouest (FEW ib.).

\section{SUFFIXATION}

\section{En-ailler}

brouscailler (Ms. 1 Cahier B). Attesté en Normandie et en Saintonge sous la forme brusquailler (FEW 1, 575b sous bruscum; MussSaint). Pour une étude détaillée du suffixe -ailler, v. TLF. Consigné dans Glossaire (sous brousquâiller et brusquâiller).

\section{En -eter}

chienneter (Ms. 1 Cahier B; Ms. 2). Forme attestée en français de 1573 à 1665; relevée en normand et en wallon (FEW 2/1, 192a sous canis). Figure dans Dionne et Glossaire. Recueillie lors d'enquêtes orales dans les années 1970 (Massicotte IV-351; PPQ653; Lavoie 1452).

empocheter (Ms. 1 Cahier B). Empocheter a été relevé en normand, en lorrain et en wallon (FEW 16, 641b sous * pokka). Consigné dans Dionne et Glossaire. Recueilli dans les années 1970 , mais moins fréquent que empocher (Lavoie 1108).

rapiester (Ms. 1 Cahier B; Ms. 2). Pour rendre compte de la prononciation,Viger, qui avait d'abord écrit rapiéceter, l'a remplacé par rapiester. Rapiéceter est attesté depuis 1624; il est bien représenté dans les parlers d'oil (FEW 8, 336a sous pettia). Rapiéceter est attesté dans les documents de la Nouvelle-France dès 1700 (JunPron, 264). Dionne l'enregistre sous rapièceter. Encore bien vivant dans les années 1970 (PPQ 323, 327; Lavoie 1724, 1726).

\section{En -ier}

balier (Ms. 1 Cahier A). Forme attestée en ancien et en moyen français; bien représentée dans les parlers d'oil (FEW 1, 232b-233a sous *banatlo). Encore retenue dans Richelet 1710, mais condamnée par Trévoux 1752 (cités dans TLF sous balayer). Platt 1835 précise : «Du temps de Ménage, on ne savait trop lequel valait mieux de balier ou balayer.» Figure dans Potier (HalPot 161). Consignée dans Dunn, Clapin, Dionne et Glossaire. Forme recueillie lors d'enquêtes orales au Québec et largement attestée (PPQ 281, 2051); connue également en Acadie (Massignon 1224), en Louisiane (ReadLouis) et à la Réunion (ChaudRéun II, 696). 


\section{En -iller}

berlanciller (Ms. 1 Cahier A; Ms. 2). Relevé en orléanais (FEW 1, 363a sous bilanx). Consigné dans Glossaire (sous balanciller et berlanciller) et recueilli lors d'enquêtes orales (PPQ2048).

écrapoutiller (Ms. 1 Cahier B; Ms. 2). Forme qui semble n'avoir été relevée qu'au Québec. La substitution de -iller à -ir est cependant attestée dans d'autres mots; cf. époutiller pour époutir en normand (FEW 9, 550a sous puls). Dionne relève s'accroupiller pour s'accroupir. Écrapoutiller est consigné dans Glossaire. Recueilli lors d'enquêtes orales dans la Beauce (LorBeauce).

\section{Résous pour Résolu}

résous (Ms. 1 Cahier $\mathrm{A}$; Ms. 2). Résous (souvent résout aux $\mathrm{XVI}^{\mathrm{e}}$ et $\mathrm{XVII}{ }^{\mathrm{e}} \mathrm{s}$.) est bien attesté, avec des variantes sémantiques, dans les parlers d'oïl (FEW 10, 303b sous resolvere; ReyHist sous résoudre). Etre résous à est attesté depuis Furetière 1690, qui donne également la forme résolu. Dupré (sous résoudre) le condamne : «Le participe résous est un archaïsme conservé artificiellement; on le laissera aux chimistes, s'ils le jugent nécessaire.» Consigné dans Dunn, Clapin, Dionne et Glossaire.

\section{VERBE DE LA PREMIÈRE CONJUGAISON}

retrayer (Ms. 1 Cahier A; Ms. 2). Retrayer pour retraire au sens de "retirern est attesté au XVe s., à la forme pronominale (FEW 10, 341a sous retrahere). Cette forme s'explique probablement par une influence analogique avec les formes du participe présent, de l'indicatif imparfait et des trois personnes du pluriel de l'indicatif présent du verbe retraire.

\section{L'ADVERBE}

même (à ) (Ms. 1 Cahier B sous à même). Mettre qqn à même "[permettre à qqn de faire qqch.; le laisser libre]»; la locution qui s'emploie, en outre, avec être et laisser est attestée en français général de 1640 à Académie 1798 (FEW 4, 807b sous ipse). «A mesme façon de parler adv. qui n'a d'usage qu'avec les verbes, Estre, mettre \& laisser. On dit, Estre à mesme, en parlant d'une personne qui aime extremement quelque chose, \& qui se trouve en estat de se satisfaire pleinement» (Académie 1694). Pour Furetière 1727, à même relève du discours familier et populaire. $\grave{A}$ même a été relevé en Anjou et en Touraine (VerrAnj; DavTour sous minme). Consigné dans Glossaire. 
Boire à même [le verre, la bouteille, etc.] est attesté en français général depuis le XIII s. (FEW 4, 807b sous ipse; Nyrop V, no 414, rem.). Viger relève cet emploi sans doute parce qu'il est donné comme populaire notamment dans Académie 1798.

Être à même de faire qqch. «être en mesure de» est attesté depuis le XVIII' s. (FEW ib.).

En résumé, les locutions être à même, boire à même qqch. et être à même de faire qqch. sont encore critiquées à la fin du XVIII ${ }^{\mathrm{e}}$ s. Féraud 1787 explique : «Mettre à même et être à même de faire quelque chôse; c. à d. mettre ou être à portée de le faire, donner ou avoir des facilités pour le faire, sont des façons de parler qui m'ont toujours paru bien bizârres, et qui certainement ne sont pas du beau style. L'Acad. dit bien être, mettre, laisser à même, mais elle les dit sans régime."

moindrement (Ms. 1 Cahiers A et B). L'adverbe moindrement, donné comme littéraire ou rare en français général, est employé de nos jours avec la négation pas : pas le moindrement, "pas le moins du monde» (ReyHist sous moindre; PRob 1993; PLar 1998). Dans des phrases affirmatives, le moindrement «le moins, très peu, un peu» et le moindrement que "aussi peu que, pour peu que» ont été relevés en Normandie (FEW 6/2,123b sous minor). Consigné dans Dunn, Clapin, Dionne; Glossaire, comme Viger, consigne également la moindrement. Encore usuel au Québec (DQA : «S'ils sont le moindrement intelligents, ils réussiront»). Outre les différences d'emploi et de sens déjà mentionnées, cet adverbe en français québécois s'écarte également de l'usage du français général par sa fréquence; il appartient, par ailleurs, au registre familier.

\section{LA PRÉPOSITION}

amont (Ms. 1 Cahier A). Amont, «vers le haut de», est attesté du XII ${ }^{\mathrm{e}}$ au $\mathrm{XVI}^{\mathrm{e}}$ s.; relevé au $\mathrm{XVI}^{\mathrm{e}} \mathrm{s}$. dans des constructions telles que amont la cheminée, amont les vignes. Amont la côte et grimper amont un arbre ont vécu en normand, en vendômois et en boulonnais notamment (FEW $6 / 3,85 \mathrm{a}-\mathrm{b}$ sous mons; HaignBoul). Consigné dans Clapin, Dionne et Glossaire. Encore recueilli en quelques endroits au cours d'enquêtes orales menées dans les années 1970 (PPQ2310:amont la pente).

\section{de pour $\grave{a}$}

affaire (avoir de qqn) (Ms. 1 Cahier B). Avoir affaire de qqn, de qqch. a cours depuis l'ancien français; donné avec la mention ufamilier" à par- 
tir d'Académie 1762 (FEW 3, 350a sous facere). Les dictionnaires qui l'enregistrent, de nos jours, lui donnent les mentions «archaïque» ou «vieux» (TLF; PRob 1993).

\section{en pour au}

[en Canada] (Ms. 1 dans le titre; Ms. 1 Cahier B et Ms. 2 sous ginseng; Ms. 1 Cahier A sous travail; Ms. 2 sous office et tuer). La règle aujourd'hui est que les noms masculins à initiale consonantique prennent l'article : Aller au Pérou, au Congo, au Canada; la préposition en est employée avec les noms masculins à initiale vocalique et les noms féminins (Grevisse $\mathrm{n}^{\circ} 318$ ). D. Bouhours dans ses Remarques nouvelles tente d'expliquer l'emploi de en et à devant les noms de pays : «On dira peut-estre que j'explique une difficulté par une autre, $\&$ on demandera enfin pourquoy Chine \& Japon conservent toûjours leurs articles contre la regle commune, qui oste quelquefois l'article aux noms de province \& de Royaume dans les cas obliques. Je répons que cette irrégularité a principalement lieu pour tout ce qu'on appelle le nouveau monde; que Cbine \& Japon ont le mesme régime que les autres païs nouvellement découverts [...]. Il faut excepter le Canada des pais : nous disons, aller en Canada; \& apparemment nous traitons ce païs-là comme les provinces de la France, parce qu'il porte le nom de France, \& que nous ne le regardons pas tout-à-fait comme le reste du nouveau monde. Aprés tout, il seroit difficile de donner une bonne raison de tout cela : aussi faut-il avoûêr que le caprice de l'usage y a plus de part que la raison; $\&$ il semble que cét usage bizarre prenne quelquefois plaisir à renverser toutes nos idées $\&$ tous nos raisonnemens» (Bouhours 11-12). En 1841, Maguire condamne l'emploi de $e n$ au lieu de au devant Canada (Maguire 12-13). En Canada, qui date du $\mathrm{XVI}^{\mathrm{e}}$ siècle, était encore en usage au début du $\mathrm{XX}^{\mathrm{e}}$ siècle.

\section{Sur «chez»}

sur (Ms. 1 Cahier A; Ms. 2). Sur au sens de "chez» est attesté en français général au XIV et au XVe s. (FEW 12, 432a sous super). Figure dans Glossaire et dans Massignon (1137).

Sus était connu également à l'époque de Viger (v. Index). Le mot est généralement prononcé [sy] de nos jours au Québec (PPQ 1743, 1744); recueilli également en Acadie (Massignon ib.). Cette prononciation provient soit de l'amuïssement de la consonne $r$ de sur, soit de sus attesté également avec l'acception de «chez» au XIV $\mathrm{e}$ s. (FEW 12, 463a sous sursum); il y aurait eu rencontre des deux mots en NouvelleFrance selon M. Juneau (JunPron 169-170; JunPMeun 186). 


\section{ASPECTS LEXICAUX}

\section{ARCHAÏSMES, DIALECTALISMES ET INNOVATIONS FORMELLES ET SÉMANTIQUES}

Nous avons toujours privilégié les définitions de Viger. Comme l'auteur ne donne pas toujours la définition du mot vedette au début de l'article, il a fallu à maintes reprises reconstituer, tout en conservant les mots qu'il emploie, les bribes de définitions qui sont éparses dans les articles. Lorsque l'auteur n'a pas donné de définition, nous en apportons une, encadrée de crochets droits.

Il nous a paru important, en outre, de tenir compte des nombreux mots oubliés et cachés qui se trouvent soit dans les définitions, soit dans les exemples des articles. Il est en effet fréquent de rencontrer dans les articles des termes qui présentent un intérêt certain aujourd'hui, mais qui n'ont pas été traités par Viger parce qu'ils faisaient partie du français de son époque, consigné dans les dictionnaires. Ces mots sont mis entre crochets droits, pour bien montrer qu'il s'agit d'ajouts dans la nomenclature, et ils ont été intercalés à leur rang alphabétique.

D'autre part, un certain nombre de variantes, de synonymes ou d'équivalents du mot vedette ont aussi fait l'objet de commentaires linguistiques; ces derniers ont été placés à la suite de l'étude historique du mot vedette et ils sont facilement repérables dans l'Index.

abât (Ms. 1 Cahier A; Ms. 2); abât de pluie, abât de neige, «grande pluie, fréquens orages; grande quantité de neige». Le mot est attesté en français dans le sens de "forte pluie" depuis Littré (sous Abat ou abas) : «averse, pluie abondante». Relevé avec les mentions «vieilli», "vieux» ou "régional» depuis le début du XX ${ }^{\mathrm{e}}$ s. (DG : des abats d'eau, de grêle; GRob; ReyHist sous abattre). Dans cette acception, abat est attesté notamment dans les parlers de l'Ouest, du Nord-Ouest et du Centre où il est encore vivant (FEW 24, $17 \mathrm{~b}$ sous abbattuere; RézOuest et SimTour : abât d'eau). Les syntagmes abat d'eau, abat de pluie, abat de neige ont été recueillis dans les années 1970 au Québec (PPQ 1175, 1207); ils semblent sortis de l'usage aujourd'hui. Abat d'eau et abat de pluie ont été relevés en Acadie (Massignon 79). V. aussi Aspects phonétiques et Datations.

âbre (Ms. 1 Cahier A). V. Aspects phonétiques. 
acquet (Ms. 1 Cahier B); «[avantage, profit, gain]». Dans ce sens, acquêt est attesté en français dès le XII ${ }^{e}$ s.; il est donné comme familier à partir d'Académie 1740 (FEW 24, 111a sous acquirere). Féraud, qui cite Académie : «Vous aurez plus d'acquêt de le payer que de le plaider», ajoute : «Cette dernière phrase me paraît surannée.» Acquêt "profit, manière de tirer parti d'une chose" a été relevé dans les parlers du Centre (FEW ib.). Le mot est consigné dans Glossaire (sous acquette); il a été recueilli lors d'enquêtes orales dans les années 1970 (PPQ 2310; LorBeauce). V. Quitte (avoir plus de ).

affaire (avoir de qqn) (Ms. 1 Cahier B). V. Aspects syntaxiques.

ahurissant, ante (Ms. 1 Cahier A; Ms. 2); «ennuyant». En français général, l'adj. aburissant, qui n'est attesté que depuis 1866 (GLLF), a pris le sens du verbe ahurir dont il dérive : «déconcerter complètement en étonnant ou en faisant perdre la tête» (PRob 1993). L'acception que donne Viger vient probablement de la Normandie où le verbe aburir a été relevé avec le sens atténué d'uétourdir, ennuyer» (FEW 4, 517a sous "bura), sens qui a vécu en français québécois (Clapin; Glossaire le donne sous aheurir). Aburissant, adj. et subst., est consigné dans Glossaire (sous agurissant, abeurissant, aburissant, ayurissant). À notre connaissance, ce sens a disparu de l'usage actuel. V. aussi Datations.

[aimable] (Ms. 1 Cahier A sous désapointer); «[mondain, homme de cour, personne qui fait l'aimable]", attesté depuis 1734 (FEW 24, 382a sous amabilis). Bescherelle 1858 explique ainsi l'emploi substantivé : «On a dit substantivement, Les aimables de la cour, c'est-à-dire, Ceux qui s'y piquaient de passer pour aimables, pour agréables. Aujourd'hui on le dit ironiquement des jeunes gens qui mettent beaucoup d'importance au talent de plaire par des qualités frivoles.» Cet emploi a disparu (ReyHist).

Allemand (être, avoir une tête d' ) (Ms. 1 Cahier A); «être entêté, opiniâtrem. L'expression est absente des ouvrages consultés. Cf. cependant l'expression argotique tête de boche «tête dure», attestée depuis 1862 (TLF sous boche) et tête carrée "personne entêtée; sobriquet de l'Allemand» depuis 1861 (FEW 13/1, 272b sous testa).

allumer (Ms. 1 Cahier A; Ms. 2); "[mettre le feu au tabac d'une pipe; au figuré, prendre un moment pour se reposer ou bavarder (et en profiter pour fumer)]». En emploi transitif, allumer une pipe est attesté depuis Bescherelle 1845 (FEW 24, 340a sous *alluminare). En emploi absolu, allumer, dans ce sens et dans le sens figuré, ne semble avoir été relevé qu'en français canadien. Consigné dans Clapin 344 ( $[\mathrm{s}]$ 'emploie souvent elliptiquement, pour "allumer la pipe"»), Dionne («se reposer»), Glossaire. Recueilli encore dans les années 1970 (PPQ 2070 «fumer»; 
Lavoie 816 «prendre quelques minutes de repos» et 3131 «fumer»). Connu en Acadie (PoirAcad). V. aussi Datations.

amancher (Ms. 1 Cahier A; Ms. 2); «raccommoder; refaire». Le sens général d' «arranger, réparer, fixer, ajuster qqch.", qui est une extension du sens français de "munir d'un manche», est attesté (surtout sous la forme emmancher) dans les parlers du Nord-Ouest, de l'Ouest et du Centre, ainsi qu'en wallon (FEW 6/1, 223a sous manicus). Présent dans Glossaire (sous amancher et emmancher). Cet emploi est également connu en Acadie (Massignon 1160). V. aussi Aspects morphologiques.

amarrer (Ms. 1 Cahier A); «lier, attacher [dans un sens général]». Cette acception est bien attestée dans les parlers du Nord-Ouest et de l'Ouest (FEW 15/1, 2b sous *aenmarren). Le mot dans ce sens a été recueilli au Québec dans les années 1970 et s'applique alors surtout aux animaux : amarrer les bêtes, amarrer un cheval (Massicotte IV-16; PPQ 390 et 422; Lavoie 1230). Relevé en Acadie (Massignon 865, $907 ; 1659$ «attacher ses souliers»), en franco-ontarien (LemVieux 20, 85) et en Louisiane (ReadLouis). Il est connu également à la Réunion, à Haïti et à la Martinique (ChaudRéun II, 681).

amont (Ms. 1 Cahier A). V. Aspects syntaxiques.

[après-dinée] (Ms. 1 Cahier A et Ms. 2 sous plaisant et relevée); "[aprèsmidi]». Le mot vit en français général depuis le début du $\mathrm{XV}^{\mathrm{e}} \mathrm{s}$. (FEW 3,95a sous disjejunare). Littré enregistre les formes après-dìnée, après-dìner, après-dîné, «temps depuis le dîner jusqu'au soir» et ajoute : «Des grammairiens ont accusé l'Académie d'avoir gardé après-dînée, parce que la dînée étant un repas fait en voyage ne peut exprimer le dîner ordinaire. Mais l'Académie n'a fait ici qu'enregistrer un usage qu'il n'est plus loisible de changer.» Le mot est de nos jours vieux ou régional (GRob). Recueilli lors d'enquêtes orales au Québec dans les années 1970 (Lavoie 27). V. Relevée.

araignée (Ms. 1 Cahier $\mathrm{A}$ ). V. Aspects morphologiques.

argent (Ms. 1 Cahier A). V. Aspects morphologiques.

arignée (Ms. 1 Cahier A). V. Aspects phonétiques.

arse (Ms. 1 Cahier B; Ms. 2); «place, espace». Arse est probablement une variante phonétique du mot normand airse : avoir l'airse, «avoir le temps, la facilité de faire une chose» (MoisyNorm). La forme airse est probablement issue du croisement des mots aire et aise. Aire a depuis le XII' s. le sens général d'«espace libre» (FEW 25, 160b sous area). Aise a également été relevé dans le sens d'«espace large» (FEW 24, $143 \mathrm{~b}$ sous adjacens). Cf. en français du XIII $\mathrm{e}$. aveir aise de, «avoir l'oc- 
casion, la facilité (de faire qqch.)», et, avec la même acception au $\mathrm{XV}^{\mathrm{e}}$ s., avoir l'aise; relevé également en Normandie (FEW 24, 144a). Consigné dans Dunn, Clapin et Dionne (qui le donnent aussi sous arce), Glossaire. Avoir [dlars] ou [dlas] est bien attesté, encore de nos jours, dans les régions de Charlevoix, du Saguenay et du Lac-SaintJean (Lavoie 1914). V. également Aspects phonétiques.

attisée (Ms. 1 Cahier B); "[feu vif fait avec une bonne quantité de bois]». Le mot est attesté dans les parlers du Nord et du Nord-Ouest (FEW $13 / 1,358$ a sous titio; ReyHist sous attiser : mot picard, «bois pour le feu" et "grand feu»). Recueilli partout au Québec lors d'enquêtes orales en 1970 (PPQ 79; Lavoie 2026) et encore bien vivant (DQA sous attiser). Relevé également en Acadie (Massignon 1204).

avisse (Ms. 1 Cahier A). V. Aspects phonétiques.

bagage (Ms. 1 Cahier B); «ménage [meubles, objets domestiques]». «Pendant longtemps, bagages a désigné le matériel de l'armée, l'équipement, les ustensiles et, avec une valeur collective au singulier, l'équipage de guerre d'une armée» (ReyHist). Furetière 1690 l'applique à des particuliers : «On dit aussi, qu'on a du bagage logé chez soy, quand on y a logé des filous, ou gens de mauvaise vie, qui a tous moments sont contraints de demesnager, d'emporter leur bagage, leurs meubles.» Figure avec la mention «familier» dans Académie 18351932, qui le définit : «mobilier de pauvres gens». Au Québec, cette acception a été recueillie dans les années 1970 dans la région du Saguenay (Lavoie 1989 «ameublement d'une maison»).

baiser (Ms. 1 Cahier B); "retaper [«tromper, attraper»]». Extension sémantique de baiser "posséder charnellement», mot du langage familier attesté dès le $\mathrm{XVI}^{\mathrm{e}} \mathrm{s}$. Le mot est consigné dans l'argot parisien depuis 1881. Ce sens a été relevé dans les parlers du Nord-Ouest, du Centre et en Bourgogne, «tromper, duper, attraper qqn» et est attesté dès 1871 en Vendômois (FEW 1, 269a sous basiare; TLF; ReyHist ; MartVend). Connu également à la Réunion (ChaudRéun II, 703-704).

Le syntagme baiser en guedou, dans le même sens, est absent des ouvrages consultés. Guedou vient peut-être de guilledou, mot d'origine incertaine, mais qui pourrait être formé du radical de l'ancien français guiller, «tromper, séduire», et de l'adjectif doux. Guedou pourrait aussi se rattacher à la famille de gadoue, comme l'indique M. Juneau (FEW 22, 83b; JunLex 188).

Baiser en pincettes, dans le même sens, est un emploi figuré de baiser en pincettes, «baiser qqn en lui pinçant légèrement les joues». V. Pincettes (baiser en $\sim$ ). V. Retaper. 
balier (Ms. 1 Cahier A). V. Aspects morphologiques.

banal (Ms. 1 Cahier A); «taureau». Du syntagme taureau banal. L'adjectif banal, terme féodal attesté en France dès le XIII ${ }^{\mathrm{e}}$ s., a le sens de "qui appartient au seigneur». Le mot, dans son emploi substantivé, a été relevé en orléanais sous la forme banau (pour une explication de cette forme, v. étau), «taureau communal» (FEW 15/1, 51b sous *ban). Au Québec, il a été relevé sporadiquement dans les années 1970 dans la région de Montmorency (PPQ 474) et dans Charlevoix (Lavoie 1261).

Au sens d'«homme extrêmement fort et de grande taille» (par analogie avec la grande taille et la force du taureau), banal est absent des ouvrages consultés. Cf. en français général, taureau banal, "homme débauché», consigné dans les dictionnaires de Furetière 1690 à Trévoux 1752 (FEW ib.). Glossaire, qui l'enregistre, lui donne le sens d'«homme effronté».

bandon (Ms. 1 Cahier B); donner bandon aux animaux, «les laisser partir pour courir les champs». Bandon, dans metre a bandon «donner permission, licence de faire qqch.», est attesté en français, du XIII ${ }^{\mathrm{e}}$ au $\mathrm{XVII}^{\mathrm{e}}$ s. (FEW 15/1, 49b sous ${ }^{*}$ ban). Le mot est relevé en orléanais : bestes a bandon, «bétail sans garde», et en Suisse romande : a bandon "(laisser le bétail) sans surveillance» (FEW ib.).

L'emploi par extension de bandon, «saison de l'année où il est permis et d'usage de laisser aller les animaux par toute la campagne», semble n'avoir été relevé qu'au Québec. (La période des bandons s'étendait du début de mai à la saint Michel, le 29 septembre, ou au plus tard au début d'octobre).

Basque (malin comme un ; être un malin ) (Ms. 1 Cahier B); cette expression est absente des sources consultées. Probablement à rapprocher, quant au sens, de la locution un tour de Basque : une supercherie (TLF sous basque; DunBouq).

bavasser (Ms. 1 Cahier B); «parler beaucoup, confusément (Il ne se dit guères que d'une personne ivre)». Attesté en 1584 chez Montaigne au sens de «bavarder» (TLF; ReyHist). Bavasser a été relevé dans les parlers du Nord-Ouest, de l'Ouest et du Centre (FEW 1, 195a sous *baba). Consigné dans Cotgrave 1611, il est absent de la plupart des dictionnaires des XVII et XVIII ${ }^{\mathrm{e}}$ s. Bavasser est réintroduit dans les dictionnaires depuis AcadCompl 1842. Il est, de nos jours, donné avec les mentions «vieilli, péjoratif ou familier» et est souvent senti comme "régional» (TLF). Recueilli dans les années 1970 (PPQ 2241 «parler à tort et à travers»). Connu dans le même sens en Acadie (Massignon 1784). Dans l'usage actuel au Québec, bavasser est employé au sens de 
«parler de façon indiscrète, divulguer ce qui devrait être tenu secret (à l'insu de qqn)» et dans celui de «bavarder, aimer beaucoup parler» (DQA; PPQ 2238, 2241; Lavoie 2870, 2933).

beauté (une ) (Ms. 1 Cahier B); «beaucoup, un grand nombre; peu, petit nombre». Attesté en français du XIII' s. sous les formes boutee, botee avec le sens de "grande quantité» (FEW 15/1, 210b sous *botan). Cf. Godefroy : «Une boutee de roisins bons et menus».

Par antiphrase, une beauté au sens de «peu, petit nombre» est connu des parlers du Nord-Ouest et du Centre; cf., dans le Perche, boutée, «un peu» (FEW ib.). Consigné dans Clapin, Dionne; Glossaire (sous bôtée). Connu en Acadie (PoirAcad). Encore bien vivant de nos jours au Québec.

ber (Ms. 1 Cahier A); "petit lit où l'on couche les enfants à la mammelle». Attesté depuis le XII s., d'abord sous la forme bers, le mot a été évincé à partir du XVII" s. (Furetière 1690 : "On disoit autrefois bers au lieu de berceaus) par berceau, un dérivé de bers, attesté depuis le $\mathrm{XV}^{\mathrm{e}} \mathrm{s}$. Ber (ou bers) a été largement relevé dans les parlers du Nord-Ouest, de l'Ouest et du Centre (FEW 1, 337a sous *bertiare; ReyHist sous ber ou bers). TLF l'enregistre avec la mention "vieux». Ber est largement attesté dans les documents d'archives québécois (JunLex 115 et suiv., pour une étude détaillée). Encore bien vivant dans les années 1970 (PPQ 140; LorBeauce). Relevé également en Acadie (Massignon 1164).

berlancille (Ms. 1 Cahier A; Ms. 2); «corde, branche d'arbre ou planche avec quoi on se balance». Le mot a été relevé à Blois, dans l'Orléanais (FEW 1, 363a sous bilanx). Consigné dans Glossaire qui enregistre les formes berlancille et balancille. Relevé sporadiquement dans les années 1970, sous la forme balancille (PPQ 2047). V. aussi Aspects phonétiques.

Des trois autres termes donnés par Viger comme synonymes, seul balançoire est consigné sans mention dans les dictionnaires (PRob 1993; PLar 1998); escarpolette est aujourd'hui donné comme vieilli (PRob 1993), mais il est consigné sans mention dans PLar 1998; et brandilloire est archaïque (ReyHist sous brandir).

berlanciller (Ms. 1 Cahier A; Ms. 2); "se balancer ou se brandiller». Berlanciller a également été relevé à Blois (FEW 1, 363a sous bilanx). Figure dans Glossaire (sous berlanciller et balanciller). Recueilli dans les années 1970 sous les formes balanciller, barlanciller et beurlanciller (PPQ 2048). V. aussi Aspects phonétiques et Aspects morphologiques. 
Des deux synonymes donnés par Viger, seul se balancer est encore en usage. Se brandiller, attesté depuis le XVI ${ }^{\mathrm{e}}$., est consigné avec la mention «vieux» depuis Académie 1932 (FEW 15/1, 246a sous brand; TLF; ReyHist sous brandir).

berline (Ms. 1 Cahier A); «sorte de voiture d'hyver pour la commodité des voyages». Il s'agit d'un véhicule rudimentaire fait d'un long traîneau ceinturé par des panneaux verticaux qui couvrent les quatre côtés (CorrVoit 101). Extension sémantique de «voiture hippomobile suspendue, à quatre roues et à deux fonds, garnie de glaces et d'une capote» (PRob 1993), sens qui est attesté depuis Académie 1718. Le mot désigne une voiture rudimentaire en Bourgogne et en wallon (FEW 15/1, 95b sous Berlin). Au XVIII s., au Québec, berline désignait, comme en France, une "voiture d'été»; la première attestation sûre de berline, «voiture d'hiver», daterait de 1793 (JunLex 138). Consigné dans Clapin : «Voiture dont l'arrière-train est en forme de longue caisse, et qui sert spécialement aux boulangers pour le transport et la distribution du pain». Enregistré aussi dans Glossaire : «voiture pour les voyageurs» et «voiture de livraison». Recueilli dans les années 1970 : «traîneau bas à patins pleins»; le mot est généralement prononcé barline (PPQ 1101C; Lavoie 874). Connu également en Acadie (Massignon 669).

berloque (Ms. 1 Cahier $\mathrm{A}$ ); «montre sujette à aller mal». Très largement représenté, sous cette forme, dans le sens de "montre détraquée» ou «vieille montre», dans les parlers d'oil et en franco-provençal (FEW 8, 567b-568a sous "pir-). Consigné dans Dunn, Clapin, Dionne et Glossaire. Le mot a été recueilli, dans les années 1970, au sens de «chose de peu de valeur» (PPQ1791E). V. aussi Aspects phonétiques.

beurrée (Ms. 1 Cahier B; Ms. 2); «tranche de pain sur laquelle on a étendu quelque substance grasse, onctueuse ou liquide». Attesté dès le $X V I^{\mathrm{e}}$ s. au sens de «couche de beurre étendue sur le pain», et depuis 1642 dans celui de "tartine de beurre" (FEW 1, 664a sous butyrum). Beurrée, au sens large donné ici, est un apport des parlers du Nord-Ouest, de l'Ouest et du Centre (FEW ib.); encore usité en Normandie notamment (LepNorm). Le sens de "tartine de beurre» est lui-même senti, de nos jours, comme vieilli ou régional (TLF; ReyHist sous beurre; PRob 1993; PLar 1998 signale, par ailleurs, qu'il s'agit d'un régionalisme canadien. Au Québec, le mot est encore bien vivant (DQA : beurrée de beurre, de margarine, de confiture, de miel, de mélasse, etc.). Relevé en Acadie (Massignon 1315), en franco-ontarien (LemVieux 17,141 ) et en franco-américain du Missouri (DorrSteGen).

beurrer (Ms. 1 Cahier B; Ms. 2); «[enduire du pain de graisse, de confiture, etc.]». Beurrer, d'abord relevé sous la forme burer, est attesté 
depuis le XIII s., avec l'acception de «recouvrir d'une couche de beurre»; l'extension sémantique que l'on observe ici vient des parlers du Nord-Ouest (FEW 1, 664b sous butyrum). Ce sens est encore bien vivant au Québec (DQA sous beurre: «Beurrer du pain avec du miel, de la confiture"). Relevé également en Acadie (Massignon 1314).

L'emploi pronominal se beurrer (les mains, par ex.) au sens de «se salir, se tacher avec qqch. qui s'étale» est encore courant au Québec (DQA $i b$.). Recuelli en franco-ontarien (LemVieux 2, 68).

bicler (Ms. 1 Cahier A; Ms. 2); «loucher». Bicler a eu cours du XVI ${ }^{\mathrm{e}}$ au $\mathrm{XVII}^{\mathrm{e}} \mathrm{s}$.; remplacé à partir du XVII ${ }^{\mathrm{e}}$ s. par la forme bigler, elle-même donnée comme vieillie de nos jours dans le sens de «loucher» (ReyHist sous bigle). Bicler a été largement relevé en France d'oïl ainsi qu'en franco-provençal (FEW 1, 380a sous *bisoculare). Consigné dans Glossaire. Recueilli au début des années 1970 (PPQ 2095s).

bicleux (Ms. 1 Cahier A; Ms. 2); «louche [qui est atteint de strabisme]». Relevé notamment en Touraine (DavTour). Consigné dans Glossaire. Recueilli au début des années 1970 (PPQ 2095). V. Louche, Loucheur et Vire-l'cil. V. aussi Aspects morphologiques.

biner (Ms. 1 Cahier B); «avoir du dépit, enrager». Biner est un apport de la Saintonge (MussSaint : biner, «endêver, se dépiter»). Encore connu, de nos jours, dans les parlers de l'Ouest (biner, "maugréer", DubGloss). Consigné dans Glossaire. V. Corner.

[bled d'Inde] (Ms. 1 Cahier B sous coton); «maïs. Daté de 1583 dans FEW 4, 640a sous Indes et de 1603 dans ReyHist (sous blè). «Céréale originaire de l'Amérique, le maïs figure toujours dans les Mémoires et Relations de la Nouvelle France - de Champlain à Montcalm -, sous le nom de bled d'Inde, par opposition au bled froments (Massignon 721). Le mot est vieux en français général, mais figure dans les dictionnaires comme «régionalisme canadien» (ReyHist, PRob 1993 et PLar 1998 sous blé). Blé d'Inde qui entre dans de nombreux syntagmes au Québec, est le mot courant pour désigner le maïs (DulDictC; DQA sous blè).

Au figuré, avoir, donner un beau bled d'Inde, «[faire] un pied de nez», est consigné dans Glossaire (sous blé d'Inde) dans une acception voisine : pousser un blé d'Inde, "dire, lancer une injure». Pousser un blé d'Inde, "taquiner», a été recueilli dans les années 1970 (PPQ 2275).

bleuet (Ms. 1 Cahier B); «[variété d'airelle]». Désigne le Vaccinium myrtilloides et le Vacinium angustifolium. Le nom s'applique à la plante et au fruit. Attesté d'abord sous les formes bleue ou blue chez Cayet 1605, Lescarbot 1609 et Champlain 1620; Champlain 1632 écrit blue et 
bluet (cités dans Massignon 211). Les formes bleuet et bluet sont attestées en Normandie et dans les parlers de l'Est, ainsi qu'en Suisse romande où elles s'appliquent à l'«airelle noire» (FEW 15/1, 148a-b sous ${ }^{*}$ blao). Enregistré dans Bescherelle 1858 : bluet du Canada. Relevé par Potier (HalPot 238). Connu partout au Québec sous les formes bleuet, bluet et beluet (PPQ1653; Lavoie 622; DQA sous bleuet), ainsi qu'en Acadie (Massignon 211).

blonde (Ms. 1 Cahier B); «amante [au sens de «jeune fille courtisée; francée»]». Cet emploi de blonde est attesté depuis Boiste 1829. Consigné généralement dans les dictionnaires avec la mention «populaire ou familier» à partir du XIX ${ }^{\mathrm{e}}$ s. Le mot est vieilli aujourd'hui en français général. Blonde a été relevé dans de nombreux parlers d'oil ainsi qu'en Suisse romande (FEW 15/1, 170b sous ${ }^{*}$ blunda-; ReyHist sous blond, blonde). Le terme est encore connu partout au Québec où il a pris, en outre, l'acception plus étendue de «femme, jeune fille que l'on fréquente ou avec laquelle on vit maritalement; femme avec laquelle on est marié» (DQA sous blond). Relevé en Acadie (Massignon 1722) et dans les parlers franco-américains (DFQ).

Aller voir sa blonde a été relevé dans les parlers du Centre notamment (JaubCentre). Encore en usage au Québec.

Les expressions être un amoureux de 36 blondes et être fort sur la blonde sont à rapprocher, quant au sens, des nombreuses expressions formées avec le mot blonde, dont, entre autres, aller aux blondes, aller à la blonde, relevées en France dans les parlers de l'Est notamment (FEW ib.), ainsi que courtiser la brune et la blonde, "faire la cour à beaucoup de femmes», depuis Académie 1835 (FEW 15/1, 308b sous *brun).

Le mot amante que Viger donne comme synonyme de blonde est vieilli ou littéraire de nos jours (TLF). V. Amant, amante sous Cavalier.

bombarde (Ms. 1 Cahier A); "petit instrument de fer, qui a une languette au milieu dont on tire un son en le mettant entre les dents et en le touchant avec le bout du doigt». Dans cette acception, bombarde a été relevé dans les parlers du Centre, en Bourgogne ainsi qu'en francoprovençal (FEW 1, 430b sous bombus). Consigné dans TLF (qui cite Molard : bombarde ou guimbarde). Bescherelle 1858 précise : "On a aussi donné le nom de bombarde à l'instrument appelé depuis guimbarde.» Le mot guimbarde est attesté depuis 1739 (ReyHist). Bombarde est encore le terme usuel au Québec pour désigner cet instrument (PPQ 2060; Lavoie 3056; DQA). V. Trompe.

bombe (Ms. 1 Cahier A; Ms. 2); «vase dans lequel on fait bouillir l'eau pour le thé». Bombe «projectile offensif qui avait anciennement une forme sphérique» a donné en français, par analogie de forme, le sens 
ancien de «vase sphérique en verre». Attesté dans Trévoux 1752 (TLF; ReyHist). Cf. également en langue d'oc le sens de "flacon de terre rond à cou très court» (FEW 1, 431a sous bombus). La même analogie est sans doute à l'origine du sens de «bouilloire» en français québécois; attesté depuis 1779 (1766 dans bombe à thé, «théière») (DFQ 31). Recueilli lors d'enquêtes orales en 1970 (PPQ 170, qui indique que bombe est bien attesté surtout dans l'est du Québec; Lavoie 2132). Le mot est vieilli de nos jours au Québec. Attesté en Acadie (Massignon 1267). V. Canard.

Le mot bouilloire, dans le même sens, est attesté depuis Académie 1740 (ReyHist). Il était peu usité au Québec à l'époque de Viger, et celui-ci précise : «Le dernier de ces mots [bouilloire] n'est pas du tout en usage, quoiquil soit celui dont on devroit se servir.» Mot usuel de nos jours, l'arrivée de la bouilloire électrique l'ayant confirmé dans l'usage général.

bord (Ms. 1 Cahier B); «verso d'un livre; côté». Cet emploi de bord découle du sens nautique de «côté d'un navire» (FEW 15/1, 180a sous *bord; ReyHist). Au sens large de "côté», bord est un apport des parlers du Nord-Ouest et de l'Ouest (FEW ib.). Relevé également par Potier (HalPot 238). Usuel au Québec où il se substitue à côté dans de nombreux contextes (DQA). Connu en Acadie (Massignon 1143) ainsi que dans les parlers franco-américains de la Louisiane (DitchyLouis) et du Missouri (DorrSteGen).

bordée (Ms. 1 Cahier A; Ms. 2); bordée de neige, "[chute de neige abondante]». En français, bordée, terme de marine dérivé de bord, est attesté depuis 1546. Le sens métaphorique de "grande quantité, salve» (bordée d'insultes, d'injures, depuis Académie 1762) provient de l'ancien sens collectif de "pièces d'artillerie rangées sur chaque bord d'un vaisseau» (ReyHist sous bord). Attesté en Saintonge : bordée de pluie, de grêle, «grande quantité» (FEW 15/1, 181a sous *bord), ainsi que chez Georges Duhamel : quelques bordées de grêlons (TLF). Relevé par Potier à Lorette en 1744 (HalPot 156). Toujours bien vivant au Québec (DQA). Bordée de neige est attesté en Acadie (Massignon 108, qui donne comme première attestation la date de 1727). Le mot est connu en français de la Réunion au sens de "grande quantité" (ChaudRéun II, p. 708).

bordel (Ms. 1 Cahier A); «sorte de voiture d'hyver pour la commodité des voyages». Le bordel étant une voiture rudimentaire faite de planches et de madriers, on peut penser que le terme est un dérivé de bord, lequel a donné les mots bordages, bordaille, bordé au sens d'uensemble des planches qui revêtent le corps d'un bâtiment» (FEW 15/1, 181b sous "bord); l'influence de bordel au sens de "cabane, maisonnette", lui- 
même issu de bort, «planche, d'une certaine grosseur, comme membrures et madriers", n'est peut-être pas à écarter et pourrait expliquer la substitution de suffixe (FEW 15/1, 187a sous bord; ReyHist). Ce mot qui semble n'avoir été relevé que par Viger a disparu.

boucane (Ms. 1 Cahier A; Ms. 2); «fumée». Déverbal de boucaner au sens de "fumer de la viande, du poisson pour les conserver". Boucane a été relevé en Normandie et en Saintonge avec l'acception de «fumée (épaisse)» (FEW 20, 72b sous mokaém). Le mot appartient au langage familier de nos jours au Québec (DQA sous boucan 2). Relevé en Acadie (Massignon 1210) ainsi que dans les parlers français des ÉtatsUnis (McDermMiss; DorrSteGen).

boucaner (Ms. 2); v. tr., «faire secher du poisson et de la chair au soleil ou à la cheminée, comme font les Sauvages». Attesté depuis 1575, boucaner est un dérivé de boucan, mot tupi (Brésil), utilisé en français depuis 1578 et désignant le «gril de bois sur lequel les Caraibes fument leur viande» (FEW 20, 72a sous mokaém; ReyHist sous boucan). Le verbe, dans cette acception, est senti comme vieilli de nos jours au Québec (DQA).

Boucané dans viande boucanée et jambon boucané, syntagmes que donne Viger, est également vieilli (DQA).

boucaner (Ms. 1 Cahier A; Ms. 2); v. intr., «fumer [dégager de la fumée]». Extension sémantique de boucaner v. tr. (v. ci-dessus). Cet emploi a été relevé en Normandie : «fumer (d'une cheminée), fumer en flambant, fumer démesurément» (FEW 20, 72a sous mokaém). Le mot appartient au langage familier de nos jours au Québec (DQA sous boucan 2). Relevé en Acadie (Massignon 1211).

boudin (faire du ) (Ms. 1 Cahier B); «bouder». Première attestation de cette expression qui est encore usuelle dans le langage familier (PRob 1993). V. Datations.

bougon (Ms. 1 Cahiers A et B); «[bout (d'une chandelle); pipe au tuyau très court]». Hérité de Normandie où le mot désigne surtout des bouts de bois : bougon, «morceau de bois gros et court» (FEW 15/1, 174b sous bogen), sens qui a été recueilli également à l'île aux Grues (Massicotte V-129). Bougon (de pipe) figure dans Glossaire. Recueilli dans les années 1970 (PPQ 2072B; Lavoie 3135). Bougon de pipe est connu en Acadie (PoirAcad, qui signale également le terme de pêche bareng bougon "hareng dont la tête et la queue ont été coupées»). En Louisiane, bougon désigne un «épi de maïs petit et peu garni de grains» (ReadLouis).

Bougon, «homme de petite taille», figure dans FEW, qui relève son utilisation en Louisiane et qui le classe parmi les mots d'origine obs- 
cure (FEW 21, 284a). En parlant d'une personne, cf. également en bas-manceau le sens d'uenfant mal conformé" (FEW 15/1, 174b). Encore bien attesté au Québec dans les années 1970 (PPQ 2178; Lavoie 2303). Relevé en Acadie (PoirAcad) et en Louisiane (DitchyLouis). V. Nijon.

bouquin (Ms. 1 Cahier A); "plume teinte, petit cilindre, percé aux extrémités, soit d'étain, de fer, de cuivre ou d'argent, ou même un os, dont nos habitans se servent pour orner leurs pipes, ou en alonger ce qu'ils appellent le manche». Le mot désignait d'abord au XVI ${ }^{\mathrm{e}}$ s. un «bec adapté à une corne de bœuf pour en faire une trompe de chasse" (PRob 1993). Par analogie, il a été employé pour désigner l'«embouchure d'une pipe» depuis 1833; bouquin est donné comme vieilli aujourd'hui dans ce sens (TLF; ReyHist sous bouche). Recueilli dans les années 1970 (PPQ2072x; Lavoie 3039). V. aussi Datations.

bourasse (Ms. 1 Cahier B); «mauvaise humeur». Emploi figuré de bourrasque, "coup de vent très fort", attesté depuis le XVI s. sous les formes bourrache, bourrasse (GodCompl; Huguet; FEW 1, 441b sous boreas; TLF). L'emploi figuré est donné, de nos jours, comme archaique (ReyHist sous bourrasque). V. aussi Aspects phonétiques.

bourasser (Ms. 1 Cahier B); "gourmander, gronder continuellement». Bourasser est probablement à rattacher à la famille de bourre plutôt qu'à celle de bourrasque, qui a donné tardivement le verbe bourrasquer, «se livrer à des bourrasques, à des emportements", attesté depuis 1863 et donné comme néologisme par Littré (TLF). Bourasser est peut-être un héritage de l'Anjou, où le mot a le sens de abousculer, rudoyer, malmener, bourrer de coups" (FEW 1, 640b-642a sous burra; VerrAnj). Courant au Québec au sens de «brusquer, malmener, rudoyer qqn» et de «maugréer, bougonner» (DQA sous bourrasser).

bourasseur, euse (Ms. 1 Cahier B); «[qui a] l'humeur à la gronderie, [qui est] d'une humeur hargneuse». Déverbal de bourasser. Consigné dans Clapin (sous bourasseux), Dionne et Glossaire (sous bourrasseux). Relevé, sous la forme bourrasseux dans les années 1970 (PPQ 2251; Lavoie 2882; JunG1 162). Connu en franco-ontarien (LemVieux 13, 132). V. aussi Aspects morphologiques.

bourguignon (Ms. 1 Cahier $\mathrm{A}$ ); "ggros glaçons que l'on apperçoit soulevés à la surface de la rivière, lorsqu'elle est prise». Attesté dans ce sens, selon FEW, depuis Trévoux 1752 (FEW 1, 472b sous Bourgogne; TLF; ReyHist : acception spéciale et inexpliquée). Cette acception est beaucoup plus ancienne puisqu'elle est attestée dès 1613 chez Champlain : «Nous nous mismes en deuoir et passames par quantité de bourguignons, qui sont morceaux de glace separez des grands bancs 
par la violence des vents» (cité dans Massignon 133). Le mot est probablement à rattacher à l'étymon burra plutôt qu'à celui de Bourgogne (Massicotte I-123, qui donne de nombreux exemples du mot, employé sous différentes formes et acceptions et qui ont en commun le sème de "bourrelet»). Consigné dans Potier (HalPot 240). Relevé au sens de "gros glaçons sur l'eau» dans les années 1970 (Massicotte II-68 et II79; PPQ 1229; Lavoie 217). Attesté en Acadie (Massignon ib.).

boyard (Ms. 1 Cahier A); «brancard». Attesté en français, dès le XII ${ }^{e}$ s., sous les formes baiart, bayart et boyart. Le mot a été très largement relevé tant en langue d'oil qu'en langue d'oc, ainsi qu'en francoprovençal sous les formes bayart(d) et boyart(d) (FEW 1, 207b sous bajulus; ReyHist sous bard, qui mentionne les différentes hypothèses concernant l'origine du terme). Bayart (baïart) est consigné dans les dictionnaires jusqu'au début du XX $\mathrm{XX}^{\mathrm{e}} \mathrm{s}$. (TLF). Relevé dans Glossaire (sous boyart). Recueilli dans les années 1970 (PPQ2310; Lavoie 928). Attesté en Acadie (Massignon 609). V. aussi Aspects phonétiques.

brâsser (Ms. 1 Cahier B; Ms. 2); brasser le poële, «[remuer les tisons, la braise]»; brasser la poële, le chaudron, «[agiter les mets qu'ils contiennent]». Brasser est attesté dans des emplois métonymiques en français général et en français régional. Cf. dans un exemple du XVII ${ }^{e}$ s. : "Disant ceci, toujours son lit (la paillasse de son lit) elle brassait» (cité dans DG), et de nos jours en Vendée : brasser le lit, «faire le lit» (RézVend 303). Au Québec, brasser le poêle a été relevé dans les années 1970 (Lavoie 2025).

Au figuré, brasser, "savonner (réprimander)", est connu des parlers du Centre, dans un sens voisin : «traiter avec précipitation, sans ménagement et comme à tour de brass (JaubCentre). Clapin, Dionne et Glossaire ont enregistré le terme. Encore usuel de nos jours (DQA : "être l'objet d'une réprimande, se faire dire son fait. Il s'est fait brasser par la directrice»). V. Savonner. V. également Aspects phonétiques.

braye (Ms. 1 Cahier B); «instrument dont on se sert à brayer le lin, le chanvre». Attesté sous la forme broie depuis le $\mathrm{XV}^{\mathrm{e}}$ s. La variante braye est consignée dans Cotgrave 1611 et elle est connue des parlers du Nord-Ouest, de l'Ouest et du Centre (FEW 15/1, 267a-b sous *brekan). V. Aspects phonétiques.

brayer (Ms. 1 Cahier B); brayer du lin, du chanvre, «séparer les filets de la partie ligneuse». Variante de broyer; les deux formes sont attestées depuis le XV' ${ }^{\mathrm{e}}$. (FEW 15/1, 267a sous ${ }^{*}$ brekan). V. aussi Aspects phonétiques. 
bredas (Ms. 1 Cahier B); «remue-ménage». De bredasser (berdasser) (v. plus loin). Le substantif bredas (berdas), qui a pris en français québécois le sens du verbe bredasser, est peu attesté dans les parlers de France. Cf. dans le Maine : bréda, "soirée de jeu», et berdas, «niaiserie"; dans les parlers du Centre : bardadas, «grand bruit, comme d'une chose qui tombe avec fracas" (FEW 1, 540b sous brittus; JaubCentre sous bardadas). Courant encore de nos jours au Québec sous les formes barda, borda, beurda avec l'acception de «tapage, bruit» (PPQ 1840; Lavoie 2407; DQA sous barda).

Faire le bredas «faire les gros ouvrages de l'intérieur de la maison, la lessive, le blanchissage, le lavage du linge, des meubles, etc.m. Bredas, qui ne paraît pas dans cette acception sous l'étymon brittus dans FEW, vient probablement du mot bat-draps («battoir de lavandière"), formellement et sémantiquement proche, attesté également sous la forme $b a(r) d r a$; le mot a donné, en outre, en Anjou le verbe badrasser, «taper à coup de badras, du linge». Le croisement des deux mots (bre$d a s$, «remue-ménage», et $b a(r) d r a s$, «battoir»), est possible puisqu'ils viennent l'un et l'autre des parlers du Nord-Ouest et de l'Ouest (FEW 1, 252b sous barda'a et FEW 1, 295b sous battuere). Le mot a pris de nos jours, au Québec comme en Acadie, le sens général de «ménage». Il a été relevé sous les formes barda (la plus fréquente), borda, beurda et berda (PPQ 273; Lavoie 2047); figure dans DQA (sous barda). Relevé en Acadie (Massignon 1218, beurdas). V. aussi Aspects phonétiques, sous berdas, berdasser.

bredasser (Ms. 1 Cahier B); «s'occuper à des ouvrages de ménage inutiles». Le verbe, sous les formes berdasser et bredasser, est bien attesté dans les parlers du Nord-Ouest, de l'Ouest et du Centre au sens de «'occuper de choses insignifiantes; s'agiter beaucoup pour ne rien faire d'utile; remuer avec bruit» (FEW 1, 540b sous brittus; MussSaint sous beurdasser; MinPoit sous berdasser, beurdasser; JaubCentre sous berdasser; RézVoc sous bredasser). Consigné par Potier au Détroit en $1744:$ bredasser «faire mille petits ouvrage[s]» (HalPot 242). Recueilli dans les années 1970 et très répandu (PPQ278; Lavoie 810). Courant encore de nos jours (DQA sous barda). Connu également en Acadie (Massignon 1219, beurdasser, berdasser) et en franco-ontarien (LemVieux 29, 71).

Bredasser qqn «maltraiter légèrement». Le sens de «secouer qqn, remuer qqch.» est bien attesté dans les parlers du Nord-Ouest et du Centre (FEW 1, 541a sous brittus). Courant encore de nos jours au Québec (DQA sous barda : bardasser, «bousculer, secouer qqn»). V. aussi Aspects phonétiques, sous berdas, berdasser. 
bredasserie (Ms. 1 Cahier B); «tracasserie». Relevé avec la signification de «radotage» en Touraine et en Vendée (FEW 1, 541a sous brittus). Potier enregistre en 1744 bredasserie sans définition; il faut probablement lui donner le sens de «petits ouvrages» qui correspond à l'acception du verbe bredasser qu'il a défini (v. ci-dessus). Recueilli dans les années 1970 (PPQ 278s, "menus ouvrages»). Inusité ou rare aujourd'hui. V. aussi Aspects morphologiques.

bredassier, ière (Ms. 1 Cahier B); subst., «tracassier, ière». Le terme a été relevé dans les parlers du Nord-Ouest et de l'Ouest où il s'applique à une "personne bavarde, bête, brouillonne" (FEW 1, 540b sous brittus); en Poitou, «qui parle beaucoup sans rien dire» (RézVoc). Potier relève le mot sans le définir (v. plus haut bredasser et bredasserie). Dionne et Glossaire (sous berdassier) consignent le terme au sens, entre autres, de «celui qui se mêle des affaires des autres», «chicanier». Recueilli sporadiquement, dans les années 1970 , sous la forme bardassier, «qui s'agite bruyamment» (PPQ1840x, 2272x). Inusité ou rare de nos jours au Québec, la forme usuelle étant bardasseux, berdasseux. V. aussi Aspects morphologiques.

Brillant (prendre du café comme la ) (Ms. 1 Cahier B). L'expression est peut-être à rapprocher de prendre son café qui signifie «prendre du plaisir, passer un moment agréable», attestée au XIX ${ }^{\mathrm{e}}$ s. et aujourd'hui sortie d'usage (ReyExpr sous cafè). L'emploi d'un nom propre, ici Brillant, était fréquent anciennement dans les expressions en français général ; cf. notamment parler comme la servante à Pilate (NisPar 173-174).

brouscailler (Ms. 1 Cahier B); «maltraiter de paroles ou autrement». Extension sémantique du verbe brusquailler, attesté en Normandie et en Saintonge au sens de «s'agiter brusquement» (FEW 1, 575b sous bruscum; MussSaint). Relevé dans Glossaire sous les formes brousquâiller et brusquâiller. V. aussi Aspects phonétiques et Aspects morphologiques.

brousse-poil (à ) (Ms. 1 Cahier A). V. Rebrousse ou brousse-poil (à ). V. Aspects phonétiques.

brun (faire ) (Ms. 1 Cahier B); «[faire nuit]». L'expression il fait brun, «la nuit approche», est attestée du XIV $\mathrm{e}$ s. à 1863 . Connue notamment en Touraine, en Poitou, au Berry et en Lorraine (FEW 15/1, 307b sous *brun). Consignée avec la mention «familier» dans Littré et avec celle de «vieux ou littéraire» dans TLF. "La nuance de «sombre, obscur» [que comportait l'adj. brun], qualifiant la nuit, la mer, une pièce (vers 1165), est sortic d'usage au $X V I^{c} s$. mais survit dans certains dérivés archaïques ou régionaux» (ReyHist). Consignée dans Glossaire. Très bien attestée, au Québec, dans les années 1970 (Massicotte I-19; PPQ 1713A; Lavoie 30). 
brunante (Ms. 1 Cahier B); subst., «la brune, le tems entre le soleil couché et la nuit»; loc. à la brunante "sur la brune». De l'adjectif brunant, ante, «brun, de couleur brune ou sombre», formé à la fin du XII $\mathrm{e}$ s. sur le verbe brunir (ReyHist sous brun, brune). La breunant instant qui suit le coucher du soleil» a été relevé en Haut-Maine (FEW 15/1, $307 \mathrm{~b}$ sous *brun), et a la brunant "au crépuscule» en Touraine (cité dans Massignon 69). Le substantif et la locution figurent comme régionalismes canadiens dans TLF; ReyHist (sous brun, brune); PRob 1993 et PLar 1998. Le substantif et la locution sont consignés dans Dunn ("A la brunante" est une jolie expression qu'il faut conserver»), Clapin (sous breunante), Dionne et Glossaire. Encore bien vivant au Québec (DQA). Recueilli en Acadie (Massignon 69) et en francoontarien (LemVieux 1, 179). V. aussi Aspects morphologiques.

Le mot brune "tombée de la nuit; soir" que donne Viger comme équivalent français de brunante est consigné, de nos jours, avec la mention "vieux" (PRob 1993) ou "littéraire" (PLar 1998).

La locution sur la brune (depuis le $\mathrm{XV}^{\mathrm{e}} \mathrm{s}$.) que Viger donne en remplacement de à la brunante, a été évincée par à la brune attestée depuis 1643 (ReyHist sous brun, brune).

buffet (Ms. 1 Cahier A); «bureau». Le mot désigne, entre autres, en ancien et en moyen français le «bureau du greffier» (FEW 1, 598a sous buff). "Le buffet désigne, en effet, à l'origine un "étal», un "comptoir (de changeur)», une «table (de greffier)»; c'est donc un parfait synonyme de bureau [...]" (GuirÉtym).

butin (Ms. 1 Cahier A; Ms. 2); «effets, meubles, hardes d'une personne». Extension sémantique de butin, "ce qu'on prend sur l'ennemi», attesté depuis le $\mathrm{XVI}^{e}$ s. Très répandu en langue d'oïl ainsi qu'en Suisse romande, où il désigne soit l'«ensemble des biens d'une personne», soit les «vêtements», ou le «mobilier», souvent avec une valeur dépréciative (FEW 15/2, 32b-33a sous bûte). Consigné dans Dunn, Clapin, Dionne et Glossaire. Encore largement attesté dans les années 1970 (PPQ 99, «mobilier", et 1907, "vêtements»; Lavoie 1989, «ameublement d'une maison», et 2528, «vêtement»); le mot, dans ces acceptions, est vieux de nos jours. Relevé en Acadie (Massignon 1617, qui donne la date de 1646 comme première attestation de cette acception en Nouvelle-France). V. Hardes.

cabrouet (Ms. 1 Cahier A); «voiture à deux roues, longue et étroite dont nos charetiers se servent». Le mot, qui est attesté en français général depuis 1740, a été utilisé dans les Antilles au sens de "charrette dont on se sert pour le service des sucreries». Le terme est attesté dans les parlers du Nord-Ouest et de l'Ouest où il a été relevé en Normandie 
(«espèce de petite charrette sans ridelle») et en Saintonge («sorte de brouette qui sert dans le commerce à transporter les caisses, sacs, bales») (FEW 1, 375a-b sous *birotium; MussSaint). Donné comme régional dans TLF. Consigné dans Dunn (sous cabrouet : «haquet»), Clapin (sous cabarrois), Dionne (sous cabarouet) et Glossaire (sous cabarouet, caberouet, cabrouet). Recueilli au Québec dans les années 1970 au sens général de «voiture d'été à 2 roues pour transporter les marchandises» et prononcé [kabarwe], [kabarwet] (PPQ 1109A, 1110; Lavoie 912). Attesté en Acadie (Massignon 672). Le cabroutet est également une "petite voiture d'été à deux roues pour la promenade» (CorrVoit 106). Dans ce sens, le mot a été recueilli dans les années 1970 (PPQ1107A; Lavoie 903).

câdre (Ms. 1 Cahier A; Ms. 2); "peinture, tableau». De cadre «bordure entourant un tableau, une estampe». Par métonymie, le mot désigne depuis le $\mathrm{XVI}^{\mathrm{e}}$ s., en français populaire ou familier, un «tableau, une peinture»; bien attesté en France d'oil, et en Suisse romande (FEW $2 / 2,1404 a$ sous quadrus; TLF; ReyHist; Hanse : «cadre ne désigne pas un tableau, une gravure (emploi assez courant en Wallonie), mais son encadrement"). Relevé dans Glossaire. Figure dans DQA avec la mention «familier». V. aussi Aspects phonétiques.

cage (Ms. 1 Cahier B); "train de bois flottés [composé de plusieurs radeaux, se déplaçant au fil de l'eau ou tiré par un bateau]». Emploi spécialisé issu de cage au sens d' 'espace clos, généralement à clairevoie», attesté depuis le $\mathrm{XII}^{e}$ s. (TLF; ReyHist). Attesté en NouvelleFrance dès 1698 au sens de radeau : «Comme plusieurs personnes de cette ville se sont plein que ceux qui font venir du bois En Cajeu laissent ensuite les cages sur la greue, dont le public En est beaucoup Incommodé, ledit Conseil ordonne que dans 24 . heures lesdites cages seront démontées» (cité dans Massignon 312). Les auteurs qui ont relevé le terme le donnent comme synonyme de cajeux (Dunn : «Mot du crû canadien que personne ne pouvait inventer à notre place; gardons-le»; Clapin; Dionne; Glossaire; DulDictC). Recueilli lors d'enquêtes orales au Québec (PPQ 1321B; Lavoie 561) et en Acadie (Massignon ib.). V. Cajeux. V. Crible et Dram à la section Emprunts à l'anglais.

cahot (Ms. 1 Cahier A); «petite butte de neige [dans les chemins]». Cabot est attesté depuis le $\mathrm{XV}^{\mathrm{e}}$ s. au sens de "saut que fait une voiture en roulant sur un terrain mal uni» et, par métonymie, d'«aspérité de terrain provoquant les cahots» (FEW 16, 233b sous "botton; ReyHist). Dunn précise le sens : «c'est un trou dans nos chemins d'hiver, large comme le chemin lui-même, et plus ou moins long et profond, dans lequel la voiture plonge brusquement.» Le mot figure, en 1788 , dans un texte 
en anglais publié dans The Quebec Gazette (cité dans DictCan). Recueilli dans les années 1970 (PPQ1082x; Lavoie 171).

Abattre les cabots, «aplanir le chemin en coupant les petites buttes de neige». Chaque propriétaire devait entretenir sa portion de chemin : "Le cultivateur se sert d'une "gratte à neige" tirée par un cheval; cet instrument permet d'atténuer les trous, les «chaos»» (LeclVoit 70-71). Recueilli dans les années 1970 (PPQ1079:abattre, baisser, gosser, étêter, couper les cabots).

cajeux (Ms. 1 Cahier B); «trains de bois flottés». Sous la forme cageux, le terme est consigné au sens de "radeau» dans FEW, sous l'étymon cavea, daté de 1671 (FEW 2/1,553b sous cavea). Le mot est beaucoup plus ancien puisqu'il apparaît, en Nouvelle-France, dans les Relations des jésuites dès 1640 sous la forme caieux (caieul en 1649, cayeux en 1656, cajeu en 1691 : «Le Cajeu est une espèce de traîneau formé de plusieurs pièces et branches de bois liées ensemble, que l'on conduit, et sur lequel on se passe à la perche» (cité dans Massignon 313). Cajeux doit probablement être rattaché à l'étymon caio, qui a donné au $\mathrm{XV}^{\mathrm{e}}$ s. cail, "retranchement ou palissade de pieux mis dans la rivière pour préserver les moulins de la violence de l'eau» et kaye ou cay, «quai, jetée” (FEW 2/1, 46a-b sous caio; La Curne sous cay; Massignon 313). Potier relève le mot au sens de «radeau", sous la forme cajeu à Lorette en 1743 (HalPot 243). Donné comme synonyme de cage dans Dunn et Clapin (sous cageux), Dionne et Glossaire (sous cageu), DulDictC. Recueilli dans les années 1970 (Massicotte V-165; PPQ 1321B, "radeaux de billes réunis et toués par un bateau»; Lavoie 561, «train de bois», et 568, «radeau rudimentaire fait de deux ou trois billes de bois»). Relevé en Acadie (Massignon ib.). V. Cage. V. Crible et Dram sous Emprunts à l'anglais.

[calèche] (Ms. 1 Cahier A sous brancard, débarquer et ramancher); "[voiture à deux roues munie d'un siège à deux places et d'une capote mobile]». C'est le mot usuel au Québec pour désigner le cabriolet. Elle est appelée calèche canadienne pour la distinguer de la calèche européenne à quatre roues. Le mot est un emprunt à l'allemand Kalesche. Il est attesté depuis 1646 sous la forme calege (calèche depuis 1656) au sens de "voiture élégante, à quatre roues, découverte sur le devant et munie à l'arrière d'une capote de cuir qui s'abat ou se relève à volonté» (FEW $2 / 1,85 \mathrm{a}$ et $16,297 \mathrm{~b}$ sous kalesche; TLF; ReyHist). Recueilli dans les années 1970 (PPQ1107A; Lavoie 903).

[canadien] (Ms. 1 Cahier A sous bourgogner, etc.); anciennt. Subst., «[descendant de parents français né en Nouvelle-France (appelée aussi Canada)]»; adj., "[relatif aux descendants des Français venus s'établir en Nouvelle-France]». Lahontan en 1704 précise le sens du mot: 
«Canadiens, sont des naturels de Canada nez de pere \& de mere François" (Lahontan 270). Après la Conquête, le mot désignera officiellement tous les habitants du Canada sans égard à leur origine (Glossaire; DFP; DugGent 133; DQA). Le mot a cependant conservé encore longtemps son sens restrictif comme en font foi les attestations de Viger.

canard (Ms. 1 Cahier A); "vase qui sert à bouillir l'eau pour le thé». Probablement par analogie de forme avec le bec du canard; analogie présente également en français moderne, où, dans le domaine médical, on appelle canard un «bol fermé pourvu d'une long bec que l'on emploie pour donner à boire aux malades", sens attesté depuis Lar 1928 (FEW 2/1,166a sous kan; TLF). Recueilli dans les années 1970 (PPQ 170, qui montre, comme à l'époque de Viger, que canard était le mot courant dans la région de Montréal; Lavoie 2132). Comme son synonyme bombe, canard a vieilli et il est remplacé de nos jours par bouilloire, qui est le terme de la langue générale (DQA). Connu en Acadie (Massignon 1267). V. Bombe.

cannevette (Ms. 1 Cahier B et Ms. 2 sous cantine); «petit coffre divisé par compartimens, pour porter des bouteilles et des phioles en voyage». Dans cette acception, canevette n'est consigné dans les dictionnaires français que depuis 1834 (FEW 2/1,167b sous canaba) et a été relevé jusqu'au début du XX ${ }^{\mathrm{e}}$ s. comme terme maritime. Attesté également en provençal : canaveto, «coffret où l'on met les bouteilles». Le terme est beaucoup plus ancien puisqu'il est attesté à l'île Bourbon en 1727 et qu'il se rencontre dans les documents d'archives de la Réunion où il est attesté dès 1728. Selon R. Chaudenson, canevette viendrait des provinces de l'ouest de la France. En effet, le mot figure dans Musset avec l'acception de «vase en terre» (ChaudÉtude; MussSaint). Potier le relève en 1743 (HalPot 172). Canevette semble avoir disparu de nos jours au Québec. V. Cantine.

[canot] (Ms. 1 Cahier B et Ms. 2 sous canotée et canoter); "[embarcation légère, mue à la pagaie et primitivement faite d'écorce de bouleau]». De l'espagnol canoa, lui-même emprunté de l'arawak canaoa, une langue des Bahamas. La forme canot est attestée depuis 1599 (FEW $20,60 \mathrm{~b}$ sous canaoa). Sous cette forme, le mot est usité en français général dans des syntagmes figés (canot de plaisance, canot de sauvetage, canot pneumatique) (ReyHist). Canot est courant au Québec.

Aux XVIII et $\mathrm{XIX}^{\mathrm{e}}$ s., dans le vocabulaire du commerce des fourrures, le terme désigne une embarcation faite sur le modèle du canot d'écorce, mais beaucoup plus longue. A. Henry, auquel Viger s'est référé pour expliquer les termes du commerce des fourrures, écrit: "The canoes, which I provided for my undertaking, were, as is usual, five 
fathom and $a$ balf in length, and four feet and $a$ balf in their extreme breadth, and formed of birch-tree bark, a quarter of an inch in thickness" (Henry 13-14; v. également le document III des Appendices).

canotée (Ms. 1 Cahier B; Ms. 2); «tout ce qu'un canot peut contenir, un canot plein». Dérivé de canot. Canotée est absent des dictionnaires français. Relevé au Détroit, en 1744, par Potier (HalPot 156). Le mot a été relevé en anglais canadien au XVIII ${ }^{\mathrm{e}} \mathrm{s}$. et au début du XIX ${ }^{\mathrm{e}} \mathrm{s}$. (DictCan). V. aussi Aspects morphologiques.

Dans le vocabulaire du commerce des fourrures, la canotée est la «charge d'un canot d'écorce». Le mot y a en effet un emploi spécialisé $:$ il désigne un poids précis, à savoir $\ll 60$ pièces ou paquets de marchandise, du poids de 90 à 100 livres chacun, et de 1000 livres pesant de munitions de bouche” (Viger se réfere au texte de la page 15 de l'ouvrage de A. Henry, reproduit dans le document III des Appendices).

canoter (Ms. 1 Cahier B; Ms. 2); «conduire un canot; aller, se promener en canot». Dérivé de canot. Les dictionnaires donnent 1858 comme date de la première attestation de canoter (FEW 20,60b sous canasa; TLF et ReyHist sous canot). V. aussi Datations.

canoteur, euse (Ms. 1 Cahier B; Ms. 2); «qui aime ou qui sait bien canoter». Dérivé de canot. Attesté dès 1686, en Nouvelle-France, avec l'acception de "matelot en service dans un canot" (Troyes 37). Potier relève le mot dans cette dernière acception (HalPot 193). En français général, c'est canotier qui s'est imposé au sens de «matelot de l'équipage d'un canot", attesté depuis la fin du XVI ${ }^{\mathrm{e}}$ s., et "amateur montant un canot de plaisance» depuis Bescherelle 1845 (FEW 20, 60b sous canaoa; TLF). Canoteur n'a été consigné que dans GRob, qui remarque : «La forme normale est canotier, qui vieillit, à cause du sens II («chapeau»).» Canoteur est le mot courant au Québec (DFP; DQA).

cantine (Ms. 1 Cahier B; Ms. 2); «petit coffre divisé par compartimens, pour porter des bouteilles et des phioles en voyagem. Attesté, en français, de Richelet 1680 à Bescherelle 1858 (FEW 2/1, 232a sous canthus). Donné avec la mention «vieilli» dans TLF. Le mot semble sorti d'usage. V. Cannevette.

cantine (Ms. 1 Cahier B; Ms. 2); «cabaret ou taverne». Extension sémantique du mot cantine, qui désignait un "magasin fournissant les troupes en tabac (1720), puis en vin, en bière (1740)" (FEW 2/1, $232 \mathrm{a}-\mathrm{b}$ sous canthus; ReyHist). Au sens général que lui donne Viger, c'est-à-dire «lieu où l'on se réunit pour boire», cantine ne semble pas avoir été relevé ailleurs. En français moderne, on trouve cependant 
cantine, «lieu où l'on sert à boire et à manger pour une collectivité» (ReyHist). Au sens qu'il a chez Viger, cantine ne vit plus au Québec.

Les mots cabaret, attesté depuis le XIII ${ }^{\mathrm{e}} \mathrm{s}$, au sens de «lieu où l'on se réunit pour boire et pour jouer", et taverne, attesté depuis le $\mathrm{XII}^{\mathrm{e}} \mathrm{s}$. au sens de «lieu public où l'on mange et où l'on boit en payant», sont considérés comme «vieux» de nos jours (ReyHist; PRob 1993; PLar 1998).

cantinier, ière (Ms. 1 Cahier B; Ms. 2); "[personne] qui vend, ailleurs qu'à l'armée, de la boisson au détail». Dérivé de cantine. Extension sémantique de cantinier, ière, "personne qui tient une cantine, à l'armée», mot attesté depuis Académie 1762 et donné comme vieux de nos jours (FEW 2/1, 232b sous canthus; ReyHist sous cantine; PRob 1993). N'est plus en usage au Québec de nos jours.

Cabaretier, depuis le XIV es.; il est attesté presque exclusivement en picard et en wallon jusqu'au $X V I^{e}$ s. Le mot a eu cours surtout au $\mathrm{XVII}^{\mathrm{e}}$ et XVIII ${ }^{\mathrm{e}} \mathrm{s}$. (ReyHist sous cabaret). Tavernier est attesté depuis le XIII' $\mathrm{s}$.; comme cabaretier, il est aujourd'hui sorti d'usage en français général (ReyHist sous taverne). Détailleur, de sens plus général que les deux termes précédents, est attesté dès le XIII ${ }^{\mathrm{c}}$ s. (FEW 13/1, $46 \mathrm{~b}$ sous taliare). Littré, qui l'enregistre encore, écrit : «celui qui vend en détail; aujourd'hui on dit plutôt détaillant» (Littré; TLF sous détailler; ReyHist sous tailler).

capuche (Ms. 1 Cahier B; Ms. 2); «chapeau, bonnet de femme». Variante picarde de capuce ou capuchon. Le mot est consigné dans les dictionnaires depuis Littré 1863, au sens de «coiffe de femme en forme de capuchon se prolongeant sous forme de pèlerine» (Littré; ReyHist). Il a été relevé dans les parlers du Nord et du Nord-Ouest au sens de «capuchon" (FEW 2/1, 277a sous cappa). Pour Viger, le mot désigne une coiffure d'été (capuche de paille) et d'hiver (capuche de castor). À la fin du $\mathrm{XIX}^{\mathrm{e}}$ s., le mot semble surtout désigner une "coiffure légère" (Dunn : «capine d'été»; Clapin : «coiffure de femme en forme de capuchon, plus légère qu'une capine, et se portant durant la belle saison»; Dionne : «bonnet de nuit à l'usage du sexe»). Recueilli dans les années 1970 (PPQ 1966 et 1924x, «capuchon»; 1946, «bonnet de laine»; Lavoie 2632, «capuchon de la pèlerine»). Courant au Québec au sens de "capuchon" (DQA). Relevé en Acadie (Massignon 1682).

cariole (Ms. 1 Cahier A); «voiture d'hyver des villes et des campagnes, pour le plaisir de la promenade ou l'utilité des voyages». Carriole désigne, en français général, une "petite charrette campagnarde» (ReyHist sous carriole). De l'ancien provençal carriol, "chariot". Relevé en Normandie au $\mathrm{XVI}^{\mathrm{e}} \mathrm{s}$., au sens de «voiture à quatre roues» 
et depuis 1669 avec l'acception de «charrette à deux roues» (FEW 2/1, 435a sous carrus). Carriole, «voiture d'hivern, est une extension du sens français attestée en Nouvelle-France à la fin du XVII s. (JunPMeun 111 n. 27). Consigné dans Dunn : «En frangais Voiture à roues. Ici, Voiture d'hiver à un seul ou deux sièges, composée d'une boite placée sur deux patins très bas et en bois solide.» Il figure aussi dans Clapin, Dionne et Glossaire (sous carriole). Recueilli dans les années 1970 : «voiture d'hiver pour la promenade» (PPQ1101D; Lavoie 873, 874). Encore courant de nos jours (DQA ; CorrVoit). Relevé en Acadie (Massignon 669).

cartron (Ms. 1 Cahier A). V. Aspects phonétiques.

casque (Ms. 1 Cahier B); «bonnet de fourrure». Casque est attesté depuis le $\mathrm{XVI}^{\mathrm{e}}$ s. au sens d'«arme défensive qui couvre la tête» (FEW 2/2, $1435 \mathrm{~b}$ sous *quassicare). Le mot a en français général de nombreux emplois par analogie. Il a vécu en français populaire : casque à mèche (iron.), «bonnet de coton, bonnet de nuit» (TLF). Consigné dans Dunn, Clapin et Glossaire. Très bien attesté dans les enquêtes orales faites dans les années 1970 (PPQ 1947, «toque de fourrure», 1944, "casquette»; Lavoie 2629, «toque de fourrure", 2622, «casquette»). Courant encore de nos jours (DQA : Un casque en (de) poil: :une toque de fourrure»). Connu en Acadie (Massignon 1634, «bonnet de fourrure" et "casquette»).

casseau (Ms. 1 Cahier A); «sorte de petit meuble d'écorce ou de bois, pour mettre des fruits ou autres choses». De cassiau (XII ${ }^{\mathrm{e}} \mathrm{s}$ ), cassel (XIII ${ }^{\mathrm{e}} \mathrm{s}$ ) "petite caisse" (FEW 2/1 311b sous capsa). Au sens de "petit récipient», le mot a été relevé dans le Nord-Est (Argonne), «écuelle de bois»; en Touraine, «écuelle, récipient»; en Poitou, "plat en terre, écuelle»; en Aunis et en Saintonge, "vase» (attestations citées dans Massignon 360). Le sens de "cornet" (Glossaire, sous cassot : "Notre cassot est souvent en forme de cornet») est sans doute un apport des parlers normands, où cassot a été relevé au sens de "cornet en papier" (FEW ib.). Enregistré par Potier «boîte d'écorce» (HalPot 245). Toujours bien vivant (DQA, casseau ou cassot: «Petit récipient cubique (en bois, en carton, en plastique, etc.) pour conditionner certains produits alimentaires périssables (petits fruits, frites, etc.)川). Relevé en Acadie (Massignon 360) et en Louisiane (DitchyLouis). V. Meuble.

castonade (Ms. 1 Cahier A). V. Aspects phonétiques.

cavalier (Ms. 1 Cahier B; Ms. 2); «amant». Au XVI' s., cavalier était un titre de politesse entre gens du monde, attesté depuis Cotgrave 1611; il a pris à cette époque le sens de "celui qui accompagne une dame, qui lui fait la cour» (FEW 2/1, 5a sous caballarius; ReyHist). Consigné 
dans cette acception dans Furetière $1690:$ «Se dit aussi d'un galant qui courtise, qui mene une Dame. En cette promenade, en ce bal, chaque Dame avoit son cavalier». Le mot dans cette acception est sorti d'usage en français général. Consigné dans Dunn, Clapin, Dionne et Glossaire. Cavalier est donné comme vieux de nos jours au Québec (DQA). Relevé en Acadie (Massignon 1721).

Amant et amante sont attestés depuis le $\mathrm{XII}^{\mathrm{e}} \mathrm{s}$.; ils désignent une "personne qui aime d'amour et qui est aimée». Ce sens est donné comme "vieux" de nos jours (ReyHist sous aimer; PRob 1993; PLar 1998). V. Amante sous Blonde.

[chambre] (Ms. 1 Cahier A sous espérer); «[pièce d'une maison]». En français classique, le mot chambre désignait une "pièce contenant généralement un lit, mais où l'on recevait les visiteurs, les invités (auj. en ce sens on dit "piècem)» (DFCl). En Suisse romande, le mot chambre est encore usuel au sens de "pièce en général» (PidRom : chambre de bains, à manger, à lessive). De nos jours, en français général, le terme est réservé à la "pièce où l'on couche». Chambre a été recueilli lors d'enquêtes orales au Québec pour désigner différentes pièces (PPQ64: chambre à manger; 65 : grande chambre, "pièce qui tient lieu de salon»; 66 : chambre de bain). Relevé également en Acadie (Massignon 1141, 1142 et 1145 ).

chandelle (éviter une belle ) (Ms. 1 Cahier B); «éviter un grand malheur, un péril». Probablement par analogie avec l'expression devoir une belle, une fière chandelle à $q q n$, c'est-à-dire «devoir reconnaissance à la personne qui nous a sauvé d'un péril, d'un désastre» (TLF; DunBouq).

chardron (Ms. 1 Cahier A). V. Aspects phonétiques.

charger (Ms. 1 Cahier A); «mettre le tabac dans la pipe». Charger, «mettre la quantité de matière qui convient (par ex. sur la quenouille, dans la pipe)", est consigné dans les dictionnaires depuis Trévoux 1704 (FEW $2 / 1,415$ b sous carricare). En emploi absolu, le verbe ne semble pas avoir été relevé ailleurs qu'au Québec. Recueilli dans les années 1970 (PPQ 2070; Lavoie 3150).

chatonner (Ms. 1 Cahier B; Ms. 2); «mettre bas des chats». Attesté en français depuis 1530. Largement relevé dans les parlers du NordOuest, de l'Ouest et du Centre, ainsi qu'en franco-provençal (FEW $2 / 1,516 \mathrm{~b}$ sous cattus). Chatonner est absent des dictionnaires des $\mathrm{XVII}^{\mathrm{e}}$ et XVIII ${ }^{\mathrm{e}}$ s., évincé par son synonyme chatter, attesté depuis 1642 (FEW $i b$.). Il sera réintroduit dans les dictionnaires à partir du $\mathrm{XIX}^{\mathrm{e}}$ s. TLF qui enregistre les deux mots précise que chatter est plus rare que chatonner (TLF chatter sous chat ${ }^{1}$ et chatonner sous chaton ${ }^{1}$ ). PRob 1993 ne consigne que chatonner avec la mention «rare». Relevé 
par Glossaire. Chatonner a été recueilli au Québec dans les années 1970; il était beaucoup plus fréquent que chatter (Massicotte IV-364; PPQ 665; Lavoie 1466). Relevé en Acadie (PoirAcad sous chatouner).

chienneter (Ms. 1 Cahier B; Ms. 2); «faire des chiens». Le mot est attesté, en français, de 1573 à 1665 . Il a été relevé en normand et en wallon (FEW 2/1, 192a sous canis). Remplacé par le synonyme cbienner, attesté depuis le $\mathrm{XV}^{\mathrm{e}}$ s., mais lui-même donné comme peu usité (Laveaux; Poitevin; TLF). Les deux mots sont aujourd'hui sortis d'usage en français général. Chienneter figure dans Dionne et Glossaire. Cbienneter et chienner ont été recueillis dans les années 1970 , au Québec, avec une plus grande fréquence pour le premier terme (Massicotte IV-351; PPQ653; Lavoie 1452). V. aussi Aspects morphologiques.

clairon (Ms. 1 Cahier B); «aurore boréale». Extension sémantique de "portion du ciel qui paraît lumineuse au milieu des ombres de la nuit», sens attesté de 1687 à 1869 ; cf. en picard clairon «éclat passager de la lune ou du soleil» et «éclaircie de beau temps entre deux ondées» (FEW 2/1, 740b sous clarus). Glossaire : «S'emploie généralement au pluriel en ce sens." Encore bien attesté (surtout au pluriel) dans les années 1970 (PPQ1161; Lavoie 66); recueilli en Acadie (Massignon 68). V. Tirans.

[claque] (Ms. 2 sous mèche); «extrémité la plus déliée d'un fouet, et qui souvent est une ficelle rapportée». Extension sémantique de claque au sens de «coup donné avec le plat de la main et qui produit un bruit sec», attesté depuis le XIVes. Le mot a été relevé en Savoie avec la signification que donne Viger, celle de «mèche de fouet», et en Bourgogne avec celle de "fouet" (FEW 2/1, 727a sous klakk-).

confessionnals (Ms. 1 Cahier B). V. Aspects morphologiques.

[confortatif] (Ms. 1 Cahier B et Ms. 2 sous comfortable); «qui fortifie», terme de médecine attesté du XIII ${ }^{\mathrm{e}}$ au XIX ${ }^{\mathrm{e}}$ s. (FEW 2/2, 1044b sous confortare; ReyHist sous conforter).

cordeaux (Ms. 1 Cahier A ; Ms. 2) ; «longues rênes attachées à la bride d'un cheval attelé». De cordeau, "petite corde», d'abord attesté sous la forme cordel au XII ${ }^{\mathrm{e}} \mathrm{s}$. Cette acception a été relevée notamment dans les parlers du Nord, en Anjou, dans le Berry et en Champagne (FEW 2/1, 646a-b sous chorda). Consigné dans Dunn, Clapin et Dionne (sous cordeau), Glossaire (sous cordeaux). Donné comme régionalisme canadien dans TLF. Recueilli dans les années 1970, où il était plus fréquent que guides et rênes (PPQ 443 ; Lavoie 1555 ; LorBeauce). Courant encore au Québec (DQA sous corde). Relevé en Acadie (Massignon 913). 
Outre cordeaux, Viger enregistre courroies, qu'il condamne. Courroies est un nom générique qui sert de définissant et qui a le sens large de «bande étroite d'une matière souple et résistante servant à lier et à attacher». Guides, comme le précise Viger («guides est le mot propre»), est le terme du français général qui désigne les «courroies qu'on attache au mors d'un cheval attelé». Les rênes sont les «courroies fixées aux harnais de tête d'une bête de selle pour la diriger». Littré (sous rêne) précise l'emploi et le niveau de langue des deux équivalents : «Un cheval de selle a des rênes, un cheval de voiture a des guides. Cependant rênes se dit en tout cas dans le style noble.»

[corner] (Ms. 1 Cahier B sous ébrayer); «frapper de ses cornes (en parlant du bétail à cornes)». Corner est bien attesté en France d'oül et en franco-provençal (FEW 2/2,1192a sous cornu). Consigné dans Littré : "Éloignez-vous, mes enfants, cette vache corne.» Figure avec la mention «populaire» dans DG, qui donne se corner en parlant des animaux qui se frappent avec leurs cornes. Dans ce sens, le mot ne figure plus dans les dictionnaires usuels. Consigné dans Glossaire. Recueilli dans les années 1970, surtout à la forme pronominale en parlant des vaches qui se donnent des coups de cornes (PPQ 511; Lavoie 1274). V. Ébrayer.

corner (Ms. 1 Cahier B); «enrager, éprouver un dépit, un déplaisir grand et sensible». Il s'agit probablement d'un emploi figuré du verbe dialectal corgner, "regarder de côté, de travers, loucher», attesté dans les parlers du Nord-Ouest et de l'Ouest. Cf., en outre, l'adjectif corgne, relevé en Bourgogne et qui signifie "de mauvaise humeur" et le substantif corgne, "personne peu aimable, de mauvaise humeur», connu encore de nos jours dans les parlers de l'Ouest (FEW 2/2,1200b sous cornu; DubGloss). Relevé par Potier : «se dépiter, enrager, bouder» (HalPot 250). Ce sens n'a pas été consigné dans les principaux glossaires québécois. V. Biner.

corporal (Ms. 1 Cahier A). V. Aspects phonétiques.

côte (Ms. 1 Cahier A; Ms. 2); «éminence, hauteur et élévation». Dans son acception maritime, côte est attesté dès 1502, en gascon et depuis 1530 au sens de "partie du rivage que la mer vient battre»; depuis 1653 dans celui de «terre qui avoisine le rivage de la mer» (FEW 2/2, 1249a sous costa; ReyHist). Par extension, on a donné le nom de côte, en Nouvelle-France, aux urives des grands cours d'eau». Consigné dans Dionne (côte du nord : «la rive nord du fleuve Saint-Laurent»; côte du sud : "la rive sud du fleuve») et dans Glossaire. Recueilli à l'île aux Grues (Massicotte II-6). Courant encore au Québec (DQA). En franco-américain, les rives du Mississippi portaient également le nom de côtes (McDermMiss). 
côte (Ms. 1 Cahier B; Ms. 2); «rangée de terres concédées, suite d'habitations; (au pluriel) campagnes». Extension sémantique de l'acception québécoise de «rives des grands cours d'eau» (v. ci-dessus). Cette extension découle du fait que les terres étaient concédées en bordure des grands cours d'eau sous le régime seigneurial; le mot, dans cette acception, s'est imposé dès le $\mathrm{XVII}^{\mathrm{e}} \mathrm{s}$. Potier le relève à Lorette en 1743-1744 (HalPot 250). Dans cette acception, rang a évincé côte dans la langue courante aujourd'hui; ce dernier est cependant toujours vivant en toponymie (v. BlaisTop). Consigné dans Dunn, Glossaire; donné comme un terme historique dans $\mathrm{DQA}$. Ce sens a été relevé en Louisiane (ReadLouis).

côtes (coureur de ) (Ms. 1 Cahier B; Ms. 2); «vagabond».Coureur est attesté en français général au XV ${ }^{\mathrm{e}} \mathrm{s}$., avec l'acception de «marchand ambulant"; encore consigné au XVIII ${ }^{\mathrm{e}}$ s. avec ce sens. L'acception de «vagabond» a eu cours notamment dans les parlers du Nord-Ouest, de l'Ouest et en Bourgogne. Cf. les syntagmes coureur de champ, «batteur de campagne», coureur de chemins (FEW 2/2,1570b sous currere).

côtes (courir les ) (Ms. 1 Cahier B; Ms. 2); «aller vendre des marchandises de campagne en campagne [en parlant d'un mercier]». Suivi d'un nom de lieu, courir signifie "parcourir fréquemment" (ReyHist).

[coton] (Ms. 1 Cahier A sous sucet); «tuyau du blé d'Inde, ou sa tige, dégarnie de ses épis». Coston, «tige de laitue», est attesté du XVI ${ }^{\mathrm{e}}$ s. à Cotgrave 1611. Largement connu des parlers d'oil : «tige d'une plante; bas de la tige d'un végétal; tige dure et souvent creuse, etc.» (FEW 17, 128a-b sous ${ }^{*}$ skot). Encore employé, de nos jours, en Touraine (SimTour). Relevé dans Potier : cotons de tabac, «cotes» (HalPot 250). Consigné dans Dunn, Dionne et Glossaire. Recueilli lors d'enquêtes orales au Québec (Massicotte III-284; PPQ 892; Lavoie 1176) et en Acadie (Massignon 261). V. Sucet.

coton (Ms. 1 Cahier B); «[épi de bled d'Inde dégarni de ses grains : rafle de maïs]". Autre emploi de coton, "tige d'une plante, trognon de chou, etc.», largement attesté dans les parlers d'oil (FEW 17, 128a-b sous ${ }^{*}$ skot). Consigné dans Dunn, Clapin, Dionne et Glossaire. Recueilli dans les années 1970 (PPQ 892x). Encore usuel au Québec (DQA). $\mathrm{Ce}$ sens est également connu en Louisiane et à la Réunion (ChaudRéun II, 736). V. Sucet.

couette (Ms. 1 Cahier A; Ms. 2); «queue de cheveux que portent les hommes». Diminutif de queue au sens de «cheveux de derrière attachés avec un cordon et couverts d'un ruban roulé autour (sous l'Ancien Régime)», depuis 1765. Couette dans ce sens a été relevé en Saintonge et dans les Vosges (FEW 2/1, 525b-526a sous cauda; MussSaint). 
Consigné dans Dionne : «Autrefois l'on portait la couette dans la province de Québec.» De nos jours, en français québécois comme en français général, le mot désigne surtout une "mèche de cheveux" (ReyHist; DQA).

Couette, «lit de plume». Attesté depuis le XII ${ }^{\mathrm{e}}$ s. et très répandu partout en France d'oil et d'oc, ainsi qu'en franco-provençal (FEW culcita). Le mot qui était sorti de l'usage en français général a été repris au $\mathrm{XX}^{\mathrm{e}}$ s. pour désigner un «édredon garni de plume, de duvet ou de fibres synthétiques, recouvert d'une housse amovible" (ReyHist; PRob 1993; PLar 1998). Emploi courant également au Québec de nos jours.

coup (faire ) (Ms. 1 Cahier A sous faire coup); «aller combattre». De coup au sens d'«action humaine, surtout inattendue», attesté depuis le $\mathrm{XI}^{\mathrm{e}} \mathrm{s}$. (FEW 2/2, 865b sous colaphus). Richelet 1680 : «Ce mot au pluriel signifie qqfois combat, bataille, lieux où l'on se bat»; Furetière 1690 : "On dit en ce sens, qu'un homme va aux coups, pour dire, qu'il va aux occasions, qu'il essuye des coups de canon et les autres dangers de la guerre; qu'il va faire le coup de pistolet, pour dire, qu'il va deffier l'ennemy, qu'il va escarmoucher contre luy.» Coup était usuel, en Amérique, au XVII ${ }^{\mathrm{e}}$ s., pour désigner les attaques surprises des indigènes; cf. en 1646 : «[...] les Yroquois ne mentoient point en ce qu'ils avoient maintenu que ce n'estoit pas eux qui avoient fait le coup de cet Automne» (JournJés 53). Dans le vocabulaire de la guerre et de la

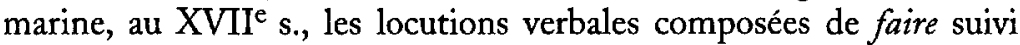
d'un substantif sans article étaient fréquentes : faire assaut, faire feu, faire sentinelle (Furetière 1690). L'absence de l'article, dans certaines locutions en français, remonte au Moyen Âge et est hérité du latin classique qui n'a pas d'articles; $\mathrm{cf}$. au X $\mathrm{XI}^{\mathrm{e}} \mathrm{s}$., sempre ferrai granz colps; également entreprendre guerre, faire combat (Nyrop IV, $\mathrm{n}^{\mathrm{Ds}} 118$ et suiv.).

couronel (Ms. 1 Cahier A). V. Aspects phonétiques.

créature (Ms. 1 Cahier B); «femme». Créature au sens de «femme» est attesté en français depuis le XII ${ }^{\mathrm{e}} \mathrm{s}$. Connu des parlers du Nord-Ouest, de l'Ouest et du Centre (FEW 2/2,1297b sous creatura). En français général, créature s'emploie encore de nos jours avec un adjectif, une belle créature ou en emploi laudatif une créature de rêve (ReyHist sous créer). Consigné dans Dunn, Clapin (sous criature), Dionne, Glossaire : «Femme, épouse, jeune fille (sans y ajouter une signification désobligeante)». Recueilli dans les années 1970 (PPQ 1733; Lavoie 2776 et 2818). Vieux de nos jours au Québec.

L'emploi péjoratif du mot créature, "femme de mauvaise vie», dans aller voir la créature, fréquenter la créature est attesté en français depuis 
le XVII ${ }^{\mathrm{e}}$ s.; il est donné comme vieux de nos jours (FEW ib.; ReyHist sous créer; PRob 1993).

Cree ou Cris (malin comme un ) (Ms. 1 Cahier B; Ms. 2). Le mot Cris (au singulier, le mot s'orthographie Cri de nos jours) désigne un membre d'une nation amérindienne du Nord canadien dont la langue appartient à la famille algonquienne. Cree est la forme anglaise.

L'expression malin comme un Cris est plutôt à rapprocher de l'expression normande méchant comme un cri, qui «se dit d'une femme acariâtre, d'un enfant indiscipliné» (FEW 16, 387a sous kriec). Consigné dans Dionne (sous cri : méchant comme un cri) et Glossaire (sous cri et cric : malin comme un petit cric (en parlant d'un enfant)). Recueilli avec la prononciation [kRik] dans les années 1970 en parlant d'un enfant (PPQ1837A; Lavoie 2740).

crocheter (Ms. 1 Cahier A); "couper [des pois] avec un petit crochet au bout d'un bâton». Crocheter, dérivé de crochet, est attesté en français depuis le $\mathrm{XV}^{\mathrm{e}}$ s. au sens de "faire agir grâce à un crochet" (ReyHist sous croc; FEW 16, 400b sous $\left.{ }^{*} k r o k\right)$. Ne semble pas avoir été relevé ailleurs dans le sens précis de "couper des pois». Consigné dans Glossaire. Recueilli dans les années 1970 (PPQ 889).

crocheteur (Ms. 1 Cahier A); «celui qui crochette [sic] des pois». Dérivé de crocheter. N'a pas été relevé ailleurs dans ce sens.

débarquer (Ms. 1 Cahier A; Ms. 2); v. tr., "[sortir qqch. d'un véhicule; descendre qqch. d'un endroit élevé]». Extension sémantique de «faire sortir (des personnes, des choses) d'un navire, mettre à terre" (PRob 1993). Le verbe est courant également au sens de «faire descendre qqn, qqch. d'un endroit élevé quelconque (un arbre, une clôture, un toit, etc.). Recueilli lors d'enquêtes orales (PPQ 2167, «faire descendre qqn de juchém, 1476, "abattre un oiseau»). Relevé en Acadie (Massignon 686, «décharger»).

Débarquer, v. intr., "descendre de voiture; descendre de cheval", est également une extension de «quitter un navire, descendre à terre» attesté depuis la fin du $\mathrm{XVI}^{\mathrm{e}}$ s. La même extension de sens est attestée en français général depuis 1713 (ReyHist sous barque). Cependant, au Québec, débarquer v. tr. et intr. se démarque du français général par sa grande fréquence et par le fait que son emploi s'étend à d'autres domaines que celui des transports (Dionne; Glossaire; MartRech 30 ; DQA). Relevé en Acadie (Massignon 674). V. Embarquer.

dégelée (Ms. 1 Cahier A); «dégel». Attesté, dans ce sens, en Normandie, en Aunis et en Saintonge (FEW 4, 87b sous gelare; MussSaint). Ce sens a disparu en français québécois. 
dégobillage (Ms. 1 Cahier A; Ms. 2); «dégobillis». Dérivé de dégobiller. Dégobillage est attesté depuis 1809 (ReyHist sous gober); il signifie «action de dégobiller» et «matières vomies» (FEW 4, 179b sous * gobbo-). Enregistré avec la mention "populaire» dans GLLF et TLF. Dégobillage est absent du PRob 1993 et du PLar 1998.

[dégobillis] (Ms. 1 Cahier A et Ms. 2 sous dégobillage); «matières vomies». Le mot est attesté en français depuis 1641 (FEW 4, 179b sous * gobbo-). Enregistré jusqu'au début du $\mathrm{XX}^{\mathrm{e}} \mathrm{s}$. dans les dictionnaires et qualifié de «mot bas, dégoûtant, trivial». TLF le donne avec la mention «populaire et vieilli».

démancher (Ms. 1 Cahier A; Ms. 2); «[défaire (une construction); défaire, découdre (un vêtement); défaire (le lit)]». Bien attesté dans les parlers de l'Ouest : "défaire (une maison), détruire" (FEW 6/1, 221a sous manicus; MussSaint); recueilli, de nos jours, en Vendée, au sens de «détricoter» (RézVend 336); connu également en wallon : «défaire, démonter, disloquer, désunir» (FEW ib.).

Démanchée, «démontée; détraquée [en parlant d'une horloge]». Cet emploi a été relevé en langue d'oil comme en langue d'oc en parlant d'une montre, d'un mécanisme quelconque qui ne fonctionne plus (FEW 6/1, 221a-b).

deusse (Ms. 1 Cahier A). V. Aspects phonétiques.

drigaille (Ms. 1 Cahier A); "[effets personnels, meubles, vêtements d'une personne]». Dans ce sens, le mot a été relevé en Poitou : «mobilier d'une ferme», «objets de ménage», «tout ce qui compose le mobilier et la garde-robe» (FEW 22/2, 86a; 23, 26a). Relevé avec une valeur dépréciative en Bretagne romane : drigailles, «objets sans valeur et un peu encombrants»; en Touraine : drigage, «choses mêlées» (FEW 23, $214 \mathrm{~b}, 234 \mathrm{~b}$ et 261a). Encore vivant en Vendée, où il signifie «accoutrement» et «ensemble d'objets indéterminés» (RézVend 327). Le mot est à rattacher au néerl. drille (FEW 25/2, 70a). Pour une étude détaillée du mot, v. JunLex 180-185. La première attestation du mot en Nouvelle-France remonte à Potier 1744 : drigail, «meubles, bagage» (HalPot 254-255). Consigné dans Dunn («Mot plaisant pour exprimer nos meubles, nos effetsm), Clapin (sous drigail), Dionne (sous drégaille), Glossaire (sous drégail, drigail). Recueilli sporadiquement dans les années 1970 (PPQ 1791x). Disparu de nos jours au Québec. Le mot a été relevé dans les parlers franco-américains (ReadLouis; McDermMiss).

ébarouir (s' ) Ms. 1 Cahier B); «[se disjoindre sous l'effet du soleil (en parlant des douves d'un tonneau, d'une futaille, etc.)]». Ébarouir, udessécher (les bordages d'une embarcation, les douves d'une futaille) de manière à les disjoindre", est consigné depuis Bescherelle 1845 (cité 
dans FEW). La forme pronominale a été relevée en Aunis et en Saintonge (FEW 15/1,71b sous *barwjan); elle figure dans LittréS. Potier l'enregistre au Détroit en 1748 (HalPot 255). Recueillie au Québec dans les années 1970 (PPQ226B).

Ébaroui est consigné depuis Corneille 1694 au sens de «desséché (en parlant de l'effet du soleil sur le bois des navires)» (FEW ib.). Relevé dans les parlers de l'Ouest en parlant des douves d'une futaille (MussSaint; RézVend 241). Ébaroui a été recueilli au Québec (PPQ 226A; Lavoie 2124) et en Acadie (Massignon 1293).

ébrâiller ([s'] ) (Ms. 1 Cahier A ; Ms. 2) ; «se découvrir l'estomac avec indécencem. Variante de se débrailler. La forme pronominale a été relevée avec la même acception dans le Limousin (FEW 1, 480a sous braca).

Ébrâillé, ée, plus usuel que le verbe, est attesté au XVI ${ }^{\mathrm{e}}$ s. au sens de «débraillé»; relevé en emploi substantivé en Saintonge et en francoprovençal : "personne dont les habits et la chemise sont ouverts par devant et découvrent sa poitrine nue» (FEW ib. ; MussSaint). Figure dans Dionne et Glossaire. Recueilli dans les années 1970 (Lavoie 2533).

L'emploi substantivé, une ébraillée, «fille indécemment mise», et par extension "fille publique», ne semble pas avoir été relevé ailleurs. $V$. Fille. V. aussi Aspects phonétiques et Aspects morphologiques.

ébrayer (Ms. 1 Cahier B; Ms. 2); «donner des coups de corne, de manière à faire mal ou dommage». De breuilles, «entrailles du poisson, de la volaille, du gibier, que l'on videm. Au sens d'eéventrer», on trouve les formes esboillier en ancien français, esbroueillier en moyen français; relevé sous celles d'ébrueiller, ébreuiller en normand. Le mot est largement attesté dans les parlers d'oil et d'oc sous différentes variantes (FEW 1, 470b-471a sous botulus). Relevé sous la forme ébreuiller par Potier (HalPot 255). Recueilli dans les années 1970, à l'île d'Orléans (PPQ 1430 : ébreiller, «vider la morue de ses entrailles»). V. Corner.

Ébrayer qqn, «battre qqn», ne semble pas avoir été relevé ailleurs. Le mot semble disparu de nos jours au Québec.

[écarquiller] (Ms. 1 Cahier B sous éjârer (s' $\sim)$ ); «écarter, ouvrir les jambes». Altération de écartiller (v. ci-dessous); attesté depuis le XVI ${ }^{\mathrm{e}}$ s. au sens général d'«écarter» : écarquiller les yeux, les jambes, les doigts (FEW 2/2, 1427a sous quartus; ReyHist). En français québécois comme en français général, écarquiller ne s'emploie de nos jours qu'en parlant des yeux : écarquiller les yeux, «les ouvrir démesurément». V. Écartiller et Éjârer (s' ). 
[écartiller] (Ms. 1 Cahier B sous éjârer (s' )); "écarter, ouvrir les jambes». Le mot date de la fin du XVI es. Écartiller est relevé dans Bescherelle 1858, Littré et DG qui renvoient à écarquiller. Écartiller a été relevé dans des parlers du Centre, en Poitou et en Bourgogne notamment (FEW 2/2, 1426a-b sous quartus). Courant encore au Québec (DQA : écartiller les yeux, les bras; s'écartiller les jambes; au fig. : être écartillé, «être partagé par deux choses contradictoires»). V. Écarquiller et Éjârer (s' ) .

écourant, ante (Ms. 1 Cahier A; Ms. 2); adj., «malpropre». Ce sens a été relevé en haut-manceau et dans des parlers du Centre. Le mot est attesté en français général au sens de «qui écœure, soulève le cœur»,

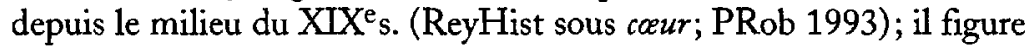
dans les dictionnaires depuis Larousse 1870 (FEW 2/2, 1172b sous cor).

L'emploi substantivé un écourant, «un malpropre», a été recueilli lors d'enquêtes orales (PPQ2273, «individu répugnant»). L'adj. et le nom s'emploient couramment au propre et au figuré au Québec (DQA sous écceurer). V. aussi Datations.

écœurer (Ms. 1 Cahier A; Ms. 2); "faire soulever le cœur, faire mal au cœur». Sous la forme esqueuré, le mot est attesté depuis Cotgrave 1611 au sens ancien de «très amaigri, affaibli». Écceurer, «affadir le couur de dégoût», date de 1642, mais il est peu usité, le mot étant considéré comme vulgaire à l'époque classique. Il s'est répandu au $\mathrm{XIX}^{\mathrm{e}} \mathrm{s}$; attesté depuis 1864 au sens de «dégoûter» et aussi d'«indigner en provoquant un dégoût moral" (FEW 2/2 1172b sous cor; ReyHist sous cour). Courant au Québec et usité au propre et au figuré (DQA).

Écceuré, ée, subst. C'est un écouré, «un dégoûté, un difficile». L'emploi substantivé est absent des sources consultées.

écolter (Ms. 1 Cahier A; s'écolter Ms. 2). V. Aspects morphologiques.

écopeau (Ms. 1 Cahier A); «éclat, morceau de bois que la hache ou quelque autre instrument tranchant font tomber du bois qu'on abat, ou qu'on met en œuvre». Variante de copeau. V. Aspects phonétiques.

L'écopeau servant à faire prendre un feu, l'expression sec comme un écopeau est à rapprocher de sec comme une allumette (Littré sous sec).

Écossois (galeux comme un ) (Ms. 1 Cahier B); Viger avait d'abord écrit fier comme un Écossois, expression courante en français général (DunBouq). L'emploi de galeux, au sens d'shomme de rien» que ce

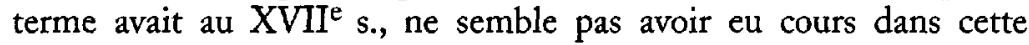
expression en français général. 
écrapoutiller (Ms. 1 Cahier B; Ms. 2). Variante d'écrapoutir (v. ci-dessous). Figure dans Glossaire; plus rare de nos jours qu'écrapoutir.

Écrapoutiller qqn comme un crapaud, «écraser qqn de ses coups». Cf. en Poitou : acrapaudai, «être aplati comme un crapaud» (FEW 16, 362b sous ${ }^{*}$ krappa).

À la forme pronominale, s'écrapoutiller (contre le mur) signifie «s'accroupir». On dit plutôt de nos jours s'écrapoutir (DQA).

Écrapoutillé, ée : doigt écrapoutillé, «écrasé»; nez écrapoutillé, «aplati, court». V. également Aspects morphologiques.

écrapoutir (Ms. 1 Cahier B; Ms. 2); «aplatir, écraser, briser par le poids de quelque chose, ou par quelque effort». Attesté en poitevin du XVI ${ }^{\mathrm{e}}$ s. : escrapoutir, «écraser». Le verbe a été relevé dans les parlers du NordOuest et de l'Ouest (FEW 16, 369b sous crasen). Figure dans Dunn, Clapin, Dionne et Glossaire. Encore vivant au Québec (DQA). Relevé en Acadie (Massignon 820).

écroi (Ms. 1 Cahier A); "petits des animaux, et particulièrement des bêtes à cornes». Dans cette acception, écroi n'a pas été relevé dans les autres parlers français. De l'ancien français escroistre, «accroître, augmenter», forme bien attestée en Normandie et qui a donné escrois au sens général et abstrait d'«accroissement (de bien, etc.)» (FEW 2/2, 1327b sous crescere). Ce dernier ouvrage relève également l'emploi canadien : écroits, «les petits d'un troupeau» (FEW ib.). Par ailleurs, le verbe accroître a donné également au sens d'«accroissement» et d'«augmentation d'un troupeau par reproduction» le terme accroit , beaucoup plus attesté (FEW 2/2,1328a). Les glossairistes qui ont enregistré le mot le donnent généralement au pluriel et dans un sens concret : Dunn (sous croit : "Le pop[ulaire] dit Les écroits, pour les petits d'un troupeau»), Clapin (sous croitts et écroits), Glossaire (sous écroît, "accroissement du bétail»). Pour Viger, écroi s'emploie aussi au singulier et désigne notamment le "petit de la vache». Le mot semble être sorti de l'usage. Relevé en Louisiane (DitchyLouis : écroit, «croît, croissance du bétail»).

[égrafignure] (Ms. 1 Cahier A sous graffigner); «blessure faite avec les ongles». Sous la forme esgrafigneure, le mot est attesté en français du $X V{ }^{\mathrm{e}}$ s.; il a été relevé dans des parlers du Nord-Ouest, de l'Ouest et $\mathrm{du}$ Centre, ainsi qu'en wallon (FEW 16, 351b sous grafla). Consigné dans Dunn, Clapin, Dionne (sous égraffignure) et Glossaire (sous égrafignure). De nos jours, au Québec comme en Acadie, la forme usuelle est grafignure (PPQ2141; Lavoie 2395; DQA sous grafigner; Massignon 1539), attestée également dans de nombreux parlers d'oïl (FEW 16, 351a). V. Graffigner. 
éjârer (s' ) (Ms. 1 Cahier B); «écarter, ouvrir les jambes». Le mot est un apport des parlers de l'Ouest et du Centre (FEW 4, 66a sous *garra). Relevé par Potier en 1746 (HalPot 160 et 256). Consigné dans Dionne (sous éjârer $\left(s^{\prime}\right)$ ) et Glossaire (sous éjarrer). Recueilli dans les années 1970 (PPQ 2161, «'étaler par terre»; Lavoie 2423, «s'étaler par terre» et 2424, «écarter les jambes»). Le mot est vieux aujourd'hui au Québec. Relevé en Acadie (Massignon 1578, «s'étaler par terre»). V. Écarquiller et Écartiller. V. aussi Aspects phonétiques.

embarquer (Ms. 1 Cahier A; Ms. 2); «monter en voiture; mettre [qqch. dans une voiture]». En français général, le verbe signifie d'abord dans son emploi transitif "faire monter (qqn) à bord d'un navire» et dans son emploi intransitif «monter à bord d'un bateau pour un voyage». Depuis le $\mathrm{XIX}^{\mathrm{e}}$ s., par extension sémantique, il s'applique à un moyen de transport quelconque (TLF). Comme pour le verbe débarquer, l'usage québécois se démarque de celui du français général par une plus grande fréquence (MartRech 30) et par ses nombreux emplois au propre et au figuré qui ne s'appliquent pas qu'au domaine des transports : embarquer sur une clotture, sur un fauteuil (en parlant d'un animal), etc.) (DQA). V. Débarquer.

empocheter (Ms. 1 Cahier B). V. Aspects morphologiques.

empois (Ms. 1 Cahier A; Ms. 2); «amidon». Emploi métonymique consigné dans Glossaire (sous empois et emplois). Recueilli dans les années 1970 (Lavoie 1714 : empois cru, «amidon de patate»). À quelques reprises, Viger a écrit emplois qui est probablement dû à l'attraction du terme emploi.

ençà (Ms. 1 Cahier A); «çà». Linterjection enfà!' a été relevée en jersiais. De fà, interjection au sens de "allons! voyons! eh bien!», généralement placée en début de phrase pour inviter, encourager, inciter à faire qqch.

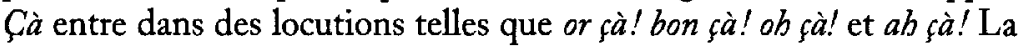
forme ençà est peut-être issue de la locution $a h$ çà! proche phonétiquement; en effet, outre la forme ansa!, le jersiais connaît également assa! Par ailleurs, une confusion avec l'adverbe de temps en çà, vieilli de nos jours, n'est peut-être pas à écarter (FEW 4, 372b sous bac; TLF sous $\grave{a}^{2}$; ReyHist sous $\mathfrak{c}^{2}$ ). Linterjection $̧ \grave{a}$ et certaines des locutions

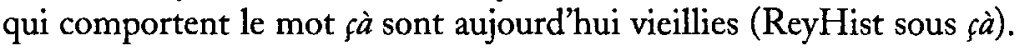

[ennuyant] (Ms. 1 Cahier A et Ms. 2 sous ahurissant); «[qui lasse l'intérêt ou la patience]». Cette forme est attestée depuis 1677; les dictionnaires de la fin du XIX ${ }^{\mathrm{e}}$ s. précisent qu'elle est moins fréquente que la forme ennuyeux (Littré) et la donnent comme familière (DG). TLF l'enregistre avec la mention «vieilli ou régional». Ennuyant a été relevé 
dans les parlers du Nord-Ouest, de l'Ouest, en wallon, ainsi qu'en Suisse romande (FEW 4, 703b sous inodiare). Forme encore très usitée au Québec (DQA sous ennuyer).

envarié, ée (Ms. 1 Cahier A). V. Aspects morphologiques.

épicailles (en donner sur les ) (Ms. 1 Cahier B); "[gronder qqn]». L'expression ne semble pas avoir été relevée ailleurs. Il s'agit d'un emploi figuré, emprunté probablement au domaine du vannage du grain. Le mot est attesté en ancien provençal : espigailh, «débris d'épis qui restent sur l'aire après qu'on a vanné le blén; surtout attesté dans les parlers de l'Ouest (FEW 12,173a sous spica). Le mot est encore connu de nos jours, dans cette région, sous la forme épigail «petit épi tombant à part» (DubGloss). Le remplacement du $g$ de épigail par $c$ $(=k)$ fournit un autre exemple de ce trait phonétique largement attesté dans les documents anciens au Québec (JunPron 151). L'expression est aujourd'hui sortie d'usage.

épinette (Ms. 1 Cahier B); «[épicéa]». Le terme sert à désigner l'épinette blanche (Picea glauca) et l'épinette noire (Picea mariana) (MVictFl 143-144). Attesté dès 1664 chez Boucher : «Il y a une autre espece d'arbre, qu'on nomme Epinette : c'est quasi comme du sapin" (BouchHist 42-43). En France, le mot est attesté au XIII ${ }^{\mathrm{e}}$ s. avec les acceptions de ubuisson épineux" et de "petite épine» (ReyHist sous épine). Relevé dans les parlers du Nord-Ouest, de l'Ouest et du Centre au sens de "petite épine», «aubépine», etc. (FEW 8, 548b sous spina; aussi 12, 178a sous pinus; JunPMeun 138-139). Relevé en Acadie (Massignon 152).

Arbre de vie et arbre du paradis ne sont pas des synonymes d'épinette comme le croit Viger; ils désignent le thuya (Thuja occidentalis). Arbre de vie est attesté de 1553 à Larousse 1876 (FEW 14, 542b sous vita; RoussAnnedda; RoussP1 159). Arbre du paradis a été relevé en 1877 (FEW 7, 615b sous paradisus).

épiochon (Ms. 1 Cahier A); «épi de blé d'Inde, ou maïs, petit, cassé, ou peu garni de grains». Diminutif de épi; relevé sous les formes épiochon et piocbon dans les parlers du Nord-Ouest, de l'Ouest et du Centre, où il s'applique à toutes sortes d'épis (FEW 12, 173b sous spica; RézVend 221, «épis plus courts que les autres»). Consigné dans Glossaire (sous épiochon et piochon). Bien attesté dans les années 1970, sous la forme épiochon et celle plus fréquente de piochon (PPQ 891 et Lavoie 1174, "petit épi de maïs; PPQ 767B et Lavoie 1058, «épi d'avoine rabougri»). Les deux formes ont été relevées en Acadie (Massignon 746). Épiochon est attesté en Louisiane (DitchyLouis). 
épotraillé (Ms. 1 Cahier B); «[qui a] la poitrine découverte». De poitrail; la forme épétrâillé a été relevée en Normandie au sens de "débraillé» (FEW 8, 109a sous pectoralis). En français général, poitrail a produit le composé familier dépoitraillé, attesté depuis 1876 (ReyHist sous poitrail). Consigné dans Clapin (sous époitrailler) et Glossaire (sous époitrâillé, épotrâillé). V. Ébrâiller et Ecolter. V. également Aspects phonétiques et Aspects morphologiques.

équilibre (être dans l' ) (Ms. 1 Cahier B). V. Inquilibre (être dans l' $)$ ).

équipage (Ms. 1 Cahier B); «[état d'une personne qui a été battue]». Le sens figuré d'état, situation" date du XVII ${ }^{e}$ s. D'abord consigné dans en bon équipage en 1636, puis en mauvais équipage, «en mauvais état», de Oudin 1640 à 1864 (FEW 17, 117a sous skipa; ReyHist sous équiper). Furetière1690 écrit : «On dit figurément en Morale, qu'un homme est en paurre, en triste équippage, lorsqu'il est mal vestu, qu'il n'a pas dequoy vivre, ou qu'il a été bien battu et maltraité.» Bescherelle 1858 précise l'usage : «Il ne se dit guère qu'en mauvaise part. Être en fort mauvais équipage, dans un triste équipage, dans un piteux équipage." Équipage est consigné dans Dionne et Glossaire au sens de "gâchis».

équiper (Ms. 1 Cahier B); «maltraiter, battre». Esquipper est attesté en ancien français avec l'acception d' «éclabousser» (Godefroy). Équiper a vécu en français général au sens de «maltraiter, railler», de Furetière 1690 à Académie 1878, qui le donne comme familier (FEW 17, 117a sous skipa). Furetière 1690 en précise le sens : "On dit figurément d'un homme qui a été blessé grievement, ou battu, ou maltraité en quelque rencontre, qu'il a été mal équippé.» Dionne et Glossaire l'enregistrent avec la valeur affaiblie qu'il a encore de nos jours de «salir qqch. (un vêtement, par ex.)» et à la forme pronominale «se salir», «se blesser».

escabeau (Ms. 1 Cahier A). V. Datations.

escargot (tête d' ) (Ms. 1 Cahier A sous tête); "personne entêtée, opiniâtre». Ne semble pas avoir été relevé ailleurs.

espérer (Ms. 1 Cahier A); "[attendre l'arrivée, la venue de qqn; absolt attendre, patienter]». Le verbe s'est introduit en français avec le sens qu'il avait en latin : "considérer (qqch.) comme devant se réaliser» (avoir espérance); ce sens s'est conservé jusqu'à nos jours (ReyHist; Nyrop IV, $\mathrm{n}^{\circ}$ 117). Il a signifié "attendre» du XII ${ }^{\mathrm{e}}$ au XIX ${ }^{\mathrm{e}}$ s.; cette acception est répandue dans de nombreux parlers d'oil et d'oc, ainsi qu'en franco-provençal (FEW 12, 164b sous sperare; ReyHist; TLF). Déjà critiquée au XVIII ${ }^{\mathrm{e}}$ s., notamment par Féraud 1787, qui écrit : «Il ne faut pas confondre espérer avec attendre, et les employer indifféremment l'un à la place de l'autre, comme on fait assez mal à propos, dans les Provinces méridionales surtout, où l'on dit : Je vous espé- 
rais, pour je vous atendais [sic].» En construction absolue, espérer est connu dans le Maine et dans le Midi (FEW 12, 165a; DepMots : Provence : Espère un peu, j’arrive). Consigné, de nos jours, avec la mention "vieilli et régional» (TLF). Classé, à tort, par Viger comme un anglicisme. Recueilli lors d'enquêtes orales au Québec (PPQ 2258) et en Acadie (Massignon 1749). Vieux de nos jours au Québec.

[estomac] (Ms. 1 Cahier A et Ms. 2 sous ébrâiller, écolter; Ms. 1 Cahier B sous pesant); "[poitrine; sein, gorge]». Le sens de "poitrine» date du $\mathrm{XII}^{\mathrm{e}} \mathrm{s}$. Très répandu en France d'oül et d'oc, ainsi qu'en francoprovençal (FEW 12, 281b sous stomachus). Consigné dans Clapin et Glossaire. Ce sens est vieux de nos jours au Québec comme en français général (ReyHist; PRob 1993). Connu en Acadie (Massignon 1527).

Au sens de «seins, gorge», estomac a été largement relevé en France d'oil, ainsi qu'en Suisse romande (FEW 12, 281b-282a). Consigné dans Clapin et Dionne. Vieux de nos jours au Québec. Connu en Acadie (Massignon 1528).

étage (premier ) (Ms. 1 Cahier A); «rez-de-chaussée». Premier étage a signifié «rez-de-chaussée» du XV' au XVII' s. en français général. Le sens moderne d'rétage au-dessus du rez-de-chaussée et, éventuellement de l'entresol» est attesté depuis Furetière 1690 (FEW 12, 240a sous stare; ReyHist). Encore courant au Québec (DQA).

étau (Ms. 1 Cahier A). V. Aspects morphologiques.

étrivant, ante (Ms. 1 Cahier B; Ms. 2); adj. et subst., «qui fait des agaceries, qui tourmente; [personne qui agace]». De étriver avec suffixe -ant. Consigné dans Glossaire. Bien attesté dans les années 1970 (PPQ 2276; Lavoie 2880). V. Aspects morphologiques.

étriver (Ms. 1 Cahier B; Ms. 2); «agacer, tourmenter par ses jeux, etc.». Attesté en français, du XII e s. à Trévoux 1704, au sens de «quereller, disputer, débattre». La valeur affaiblie de «taquiner» a été relevée en Normandie, en Champagne et dans le Maine notamment (FEW 27, $255 \mathrm{~b}-256 \mathrm{a}$ sous *strid). L'expression faire étriver, «faire enrager», est usitée, encore de nos jours, en Normandie (Orne) (LepNorm). Étriver qqn et faire étriver qqn ont été recueillis lors d'enquêtes orales au Québec (PPQ 2275; Lavoie 2881) et en Acadie (Massignon 1777). Vieillis de nos jours.

La graphie taurmenter que Viger donne sous étriver dans le ms. 1, rend probablement compte de l'étymologie. Le mot, qui est issu de tormentum, s'est prononcé anciennement tormenter en français ainsi que dans de nombreux parlers (FEW 13/2, 44b et 45 a sous tormentum). 
fanals (Ms. 1 Cahier B). V. Aspects morphologiques.

[faner, fener] (Ms. 1 Cahier A sous foin). Viger dit à propos de ce mot : «On dit ici : faner pour fener le foin». Il semble ignorer que la forme moderne est faner. Ce verbe est d'abord attesté, en ancien et en moyen français, sous la forme fener ; faner, altération de fener, est attesté depuis le XIV's. (ReyHist sous foin ${ }^{1}$ ). Fener a été relevé partout en France d'oil ainsi qu'en Suisse romande (FEW 3, 458a-b sous fenum). Potier l'enregistre : fener Le foin pour : faner (HalPot 146, 260). Fener a été relevé sporadiquement dans les années 1970 (PPQ 812x). Recueilli également en Acadie (Massignon 702).

[fanfan] (Ms. 1 Cahier A sous moucher); «enfant». Attesté depuis 1525. «Le mot ne s'est maintenu que dans le nom Fanfan la Tulipe (1819)» (ReyHist sous enfant). Recueilli au Québec dans les années 1970 comme sobriquet (PPQ1735).

fard (Ms. 1 Cahier A); «mélange de diverses viandes, ou seulement d'herbes, d'œufs et d'ingrédiens, hachés menu et assaisonnés, qu'on met dans le corps de quelques animaux, ou dans quelque autre viande». Attesté dès le XIII ${ }^{\mathrm{e}} \mathrm{s}$. en ancien français et en ancien provençal sous la forme fars. Relevé en Normandie, en Anjou et en Saintonge notamment (FEW 3, 415a sous farsus). La forme moderne farce, attestée depuis le XIII ${ }^{\mathrm{e}}$ s., représente le féminin de fars (ReyHist sous farce). Potier l'enregistre sous la forme far (HalPot 260). Consigné dans Dunn, Dionne et Glossaire (sous fars). Recueilli sporadiquement dans les années 1970 (PPQ 209C et 2310). Le mot farce qui a éliminé fard de nos jours, n'était pas fréquent au début du XIX ${ }^{\mathrm{e}}$ s., d'après Viger : «Bien peu de personnes employent ici le vrai mot.»

fâro (Ms. 1 Cahier A; Ms. 2); "gallant, petit-maître de campagne». Attesté, en français général, depuis 1743 sous la forme faraud, «celui, celle qui se pavane dans ses beaux habits"; mot dialectal qui est très bien attesté en France d'oïl et d'oc (FEW 16, 199b sous "heriwald; GuirÉtym). Figure dans PRob 1993 (sous faraud), qui indique qu'il est «vieux ou régional». Consigné dans Dunn, Clapin et Dionne (sous faraud); Clapin précise que le mot n'a pas au Canada la connotation négative qu'il a en France. Recueilli au Québec dans les années 1970 (PPQ2283 et 2285; Lavoie 2886). Vieilli de nos jours.

Beau comme un faro de campagne, «extrêmement recherché dans ses habillemens", n'a pas été relevé ailleurs.

Au sens de "galant, amoureux", faro est attesté en wallon; relevé au $\mathrm{XIX}^{\mathrm{e}}$ s. en français populaire, au sens d' «amant de cœur, maquereau» (FEW 16, 199b-200a). Consigné au sens d'«amoureux» dans Clapin, Dionne et Glossaire (sous faraud). Recueilli lors d'enquêtes orales au 
Québec (PPQ 1878) et en Acadie (Massignon 1721). Vieilli de nos jours.

farôder (Ms. 1 Cahier A; Ms. 2); «faire le faro ou la cour aux Dames, faire l'amour». Le verbe est largement attesté en langue d'oil et y signifie «se pavaner, être très élégant, etc.» (FEW 16, 200a sous *heriwald). L'emploi par extension de "courtiser» ne semble pas avoir été relevé ailleurs. Les deux sens sont consignés dans Clapin et Glossaire (sous farauder).

Faire l'amour, «courtiser», que Viger emploie dans la définition du verbe farôder a signifié "courtisen" jusqu'au XVIII ${ }^{e}$ s. L'acception «accomplir l'acte sexuel (avec qqn)», attestée depuis 1622, ne s'imposera qu'au XIX ${ }^{\mathrm{e}} \mathrm{s}$. (ReyHist).

fectif (Ms. 1 Cahier B); «certain, sûr, vrai». Il s'agit probablement d'une extension sémantique du mot effectif au sens de «réel». L'altération de la forme a été relevée à Marseille : fettif. Cf. également les formes adverbiales fectivement en poitevin et fettivament à Marseille (FEW 3, 206a sous effectus).

[fener] (Ms. 1 Cahier A sous foin). V. Faner, fener.

fenouil (Ms. 1 Cahier A). V. Aspects morphologiques.

fenouillette (Ms. 1 Cahier A; Ms. 2); «fenouil». Au sens de «fenouil», fenouillette est attesté dans le Dauphiné sous la forme fenoulhet, ainsi que dans le Gers et le Béarn (FEW 3, 454a sous fenuculum).

ferlassement (Ms. 1 Cahier B); «[bruit que fait un tissu]». De ferlasser. Le mot ne semble pas avoir été relevé ailleurs qu'au Québec. Il est consigné dans Dionne (sous farlassement) et Glossaire. Semble disparu de nos jours.

ferlasser (Ms. 1 Cahier B); «se dit du bruit que fait une étoffe roide que l'on manien. Le verbe connaît plusieurs formes. La variante ferlasser a été relevée dans le Centre et l'Ouest; ferlasser, que FEW classe parmi les mots d'origine obscure au tome $23,202 \mathrm{~b}$, serait plutôt à classer, selon P. Rézeau, sous frl- au tome 3, 815a-b (RézVoc); la forme ferdasser a été relevée dans les parlers du Nord-Ouest, du Centre et de l'Ouest, et celle de fertasser dans le Centre (FEW 3, 785a sous frictare). Les glossairistes québécois qui ont relevé le verbe le donnent sous les formes ferlasser et ferdasser; consigné dans Dunn, Clapin, Dionne et Glossaire. $V$. aussi Aspects phonétiques.

fesser (Ms. 1 Cahier A; Ms. 2); «fouetter sur le dos». Fesser vient de l'ancien français faisse, fece «bande, lien» et non de fesse. Il signifie, depuis le $\mathrm{XV}^{\mathrm{e}}$ s., «battre en donnant des coups sur les fesses» (FEW 3, 424b- 
425a sous fascia; ReyHist). Le sens général de «battre, frapper, fouetter» que l'on connaît au Québec est un apport des parlers du NordOuest et de l'Ouest; le sens de "fouetter" a été relevé notamment en Saintonge (FEW 3, 425a). Fesser, «donner le fouet», est consigné dans Bescherelle 1858. Figure dans Dionne et Glossaire. Courant au Québec au sens général de "frapper qqn; frapper sur qqch.» (DQA sous fesse). Relevé en Acadie (Massignon 1591).

fièrement (Ms. 1 Cahier A); «beaucoup, bien». Au sens de «très, extrêmement», fièrement est attesté depuis le XIII ${ }^{\mathrm{e}}$ s. Qualifié de «familier» à partir du XIX s. (FEW 3, 480b sous ferus). Bescherelle 1858 l'enregistre avec la mention «populaire» : "Cet homme est fièrement bête, fièrement dupe. Il a été fièrement puni.» Relevé en picard et en normand (FEW ib.). Figure avec la mention «vieilli» dans PRob 1993. Consigné dans Dunn et Clapin.

fignoler (Ms. 1 Cahier B; Ms. 2); «faire des dépenses d'éclat soit en habits, voitures, etc.; faire le galant». Bien attesté en France d'oil et d'oc, ainsi qu'en franco-provençal où il signifie principalement «'habiller avec recherche, faire l'élégant» (FEW 3, 564a sous finis). Figure dans Dunn, Clapin et Dionne. L'emploi pronominal a été relevé en Acadie : se fignoler, «s'attiffer, se parer avec soin» (PoirAcad).

fignoleur (Ms. 1 Cahier B; Ms. 2); «celui qui fignole». De fignoler. Relevé avec cette signification, notamment en Normandie, dans le Berry, en Poitou, en Bourgogne, ainsi qu'en wallon et généralement sous la forme fignoleux (FEW 3, 564a sous finis; RézVoc sous fignoleux). Bescherelle 1858 le définit ainsi : fignoleur, «fashionable de la campagne». Clapin et Dionne l'enregistrent au sens de «coq de village, élégant", sous la forme fignoleux. Ici encore, Viger montre sa préférence pour le suffixe -eur. V. à ce sujet bourasseur et quêteur.

[fille] (Ms. 1 Cahier A sous amancher et balier); «[servante]». Dans cette acception, fille est attesté depuis le XVII ${ }^{\mathrm{s}} \mathrm{s}$. (Molière); le mot était usuel dans des syntagmes tels que fille de ferme, fille de boutique, fille d'auberge, fille de salle, etc. (FEW 3, 517 a sous filia; ReyHist). Ce sens est vieux de nos jours au Québec comme en français général (PRob 1993; PLar 1998).

La locution fille publique (sous ébrâiller), "prostituée», est attestée depuis 1771. Figure dans PLar 1998 avec la mention "péjoratif».

fine-boutique (Ms. 1 Cahiers A et B; Ms. 2); «matois, rusé». Probablement formé sur le modèle de fin matois, fin matou, fin merle, fin renard. Au figuré, boutique qui a une connotation négative, a été employé dans l' expression cela vient de la boutique de qqn, "cela est de sa propre invention, de sa méchanceté», attestée de 1694 à 1878. Cf. 
également ouvrir boutique, ouvrir la boutique, "dire tout ce qu'on a sur le cœur» (FEW 25, 21a sous apotheca) et, en Saintonge, boutiquesse, "cancanière" (MussSaint). Glossaire consigne boutique et lui donne la signification d'«intrigant, fâcheux, importun».

Au sens d'«idiot, esprit borné», fine-boutique est employé par antiphrase.

finition (Ms. 2); «fin». Attesté une première fois dans cette acception à la fin du XIV ${ }^{\mathrm{e}}$ s.; repris au $\mathrm{XIX}^{\mathrm{e}}$ s. (1820) au sens de ulimite, achèvement» (ReyHist sous $f^{1} n^{1}$ ). A vécu également en France d'oil, ainsi qu'en Suisse romande (FEW 3, 557a sous finire; HumbGen). Consigné avec la mention «vieilli ou populaire» dans TLF. Consigné dans Dunn, Clapin et Glossaire.

flambe (Ms. 1 Cahier B); «flamme». Flambe, du latin flammula, est attesté depuis le $\mathrm{XII}^{\mathrm{e}} \mathrm{s}$; connu également sous la forme flamble du XII ${ }^{\mathrm{e}}$ au $\mathrm{XV}^{\mathrm{e}}$ s.; largement relevé en France d'oil et d'oc (FEW 3, 602a sous flammula). Consigné dans TLF avec la mention «vieux et régional». Flambe était encore bien vivant dans les années 1970 au Québec (PPQ 78; Lavoie 2027). Relevé en Acadie (Massignon 1205).

flamb(1)ant, ante (Ms. 1 Cahier B; Ms. 2); «[qui est] habillé tout en neuf». Attesté depuis le XII ${ }^{\mathrm{e}}$ s., le mot s'applique d'abord à ce qui a l'éclat du feu. $\mathrm{Au} \mathrm{XIX}^{\mathrm{e}}$ s., employé seul au figuré, l'adjectif prend en argot le sens de «beau, superbe» (1837) et celui de «richement équipé, vêtu» depuis Balzac (FEW 3, 603b sous flammula; ReyHist sous flamber). Les exemples que donne Viger ( $\mathrm{O}$ n dit d'une personne habillée tout en neuf, qu'elle est flambante. Comme il est flambant! Te voilà tout flambantı) sont donc antérieurs aux attestations du français général. Figure dans PRob 1993 avec la mention «vieilli».

La locution flambant neuf, qui est usuelle de nos jours en français général, est attestée depuis 1808 , et est donnée comme populaire. Elle a été relevée en Anjou, en Saintonge, dans le Berry, dans des parlers du Centre et en gascon où elle est généralement prononcée flambant neu (FEW 3, 603b).

La locution flambant $n u$, qui ne semble pas avoir été relevée ailleurs, a probablement été créée sur le modèle de flambant neuf, le rapprochement pouvant être facilité par la ressemblance dans la prononciation des deux mots, puisque neuf était souvent prononcé [nø] anciennement. Locution encore courante au Québec (DQA sous flambant). V. aussi Aspects phonétiques et Datations.

flandrin (Ms. 1 Cahier A); subst., "paresseux». Extension de flandrin, «personne élancée et molle». Relevé dans les parlers du Nord, dans le Berry, en Bourgogne et en Auvergne notamment avec la signification 
de «lent, flâneur, fainéant, traînard, paresseux" (FEW 3, 605b sous Flandern; ReyHist; GuirÉtym, qui décèle dans flandrin une racine provençale et le rapproche de farandel, «dégingandé»). Consigné dans Clapin (qui le donne comme adj.) et Dionne. Relevé en Acadie (PoirAcad : «S'entend, ici, plutôt d'un paresseux»).

flâner (Ms. 1 Cahier A); "paresser, faire le paresseux». Verbe d'origine dialectale, attesté une première fois en Normandie en 1638. Le mot entre en français général au $\mathrm{XIX}^{\mathrm{e}} \mathrm{s}$. (1808) (FEW 15/2, 135b sous flana; TLF; ReyHist). Flâner a probablement été introduit en NouvelleFrance par les colons normands bien avant que le mot ne soit connu en français général.

flasque (Ms. 1 Cahier A); subst., «flaque [d'eau]». Altération de flaque, forme normande ou picarde de l'ancien adj. flache au sens de "mou». Variante attestée depuis le XVe s. (BW ${ }^{5}$; GuirÉtym; ReyHist). Flasque n'étant usité que comme adj., au sens de «mou», on peut se demander si Viger, qui a par ailleurs raturé cet article, n'a pas voulu écrire flache "mare d'eau», variante très répandue dans les parlers d'oil (à l'exception des parlers normands et picards qui connaissent la forme flaque qui s'est imposée en français général) (FEW 3, 592b).

flasque (Ms. 1 Cahier A; Ms. 2); subst., "peureux». Extension du subst. flasque au sens de "paresseux»; ce dernier sens est consigné dans Richelet 1680 et Furetière 1690 et 1727.

Flasque, adj., «qui a perdu son apprêt, mollasse [d'un tissus, d'une étoffe]». Ce sens est attesté depuis 1642 (FEW 3, 593a sous flaccus).

flasquer (Ms. 1 Cahier A; Ms. 2); «avoir peur». Extension de flacher au sens de «fléchir, céder, se soumettre», attesté sous différentes formes et nuances sémantiques en France d'oil (FEW 3, 591a sous flaccus).

flasquer (fer à ) (Ms. 1 Cahier A; Ms. 2); «fer à repasser le linge». Le verbe flasquer, "repasser le linge", est un apport des parlers de l'Ouest; de flasque, «fer à repasser dans lequel on met des charbons ardents» (FEW 3, 606b et 15/2,137a sous flaska). Flasque, «fer à repasser», et flasquer (surtout dans fer à flasquer) sont bien attestés dans les documents québécois des XVII et XVIII ${ }^{\mathrm{e}}$ s. (JunPron 130 n. 38). Viger précise que le verbe n'est employé que dans fer à flasquer.

fréde (Ms. 1 Cahier A). V. Aspects phonétiques.

fréte (Ms. 1 Cahier A). L'expression fréte comme glace est attesté en parlant d'une personne dans Oudin 1640 : froid comme glace, «d'humeur froide». L'expression est connue également en Normandie avec une variante : froid comme un glaçon (LepComp 392). V. Aspects phonétiques. 
fricasser (Ms. 1 Cahier A); «dissiper [de l'argent]». Attesté dans ce sens depuis1611 (ReyHist; FEW 3, 791b-792a sous frigere); donné de nos jours avec la mention «vieux» (PRob 1993). Figure dans Potier (HalPot 265).

Le verbe fricasser dans l'expression fricasser une ramasse, une dégelée à $q q n$, «battre rudement», est peut-être une extension de fricasser au sens de "frotter d'une manière répétée», attesté en Anjou et en Bourgogne (FEW 3, 791b). Cf. également en français populaire le syntagme une fricassée de pain sec au sens de "volée de coups», attesté depuis 1808 (FEW 3, 792b). Fricasser a été relevé dans fricasser un coup de pied dans le parler franco-américain du Missouri (DorrSteGen).

Se fricasser de, "[se moquer de]». Il s'agit peut-être d'un emploi figuré de se fricasser les mains, «se frotter les mains», relevé en Anjou (FEW $3,791 \mathrm{~b}$, qui relève l'acception canadienne «se moquer de»; VerrAnj). Dans ce sens, le verbe a été consigné par Potier en 1745 (HalPot 265). Figure dans Dunn, Clapin, Dionne et Glossaire.

fripper (Ms. 1 Cahier B); «fripponner, voler, tromper qqn». Friper au sens de "voler, dérober» est attesté en français, du XVI ${ }^{\mathrm{e}}$ s. à Trévoux 1771 (FEW 3, 396b sous faluppa). Consigné dans les dictionnaires du $\mathrm{XIX}^{\mathrm{e}}$ s. au sens de evoler les écrits, les idées d'un auteur" (Bescherelle 1858; Littré). Quant au redoublement ou non du $p$ dans fripper, l'usage est encore fluctuant à l'époque de Viger : Trévoux écrit le mot avec deux $p$ tandis qu'Académie n'en met qu'un.

[fripponner] (Ms. 1 Cahier B sous fripper); «[voler]». Au sens de «voler de petites choses», il est attesté depuis 1580, et au sens d'«escroquer qqn» depuis 1585 (ReyHist sous fripon). Le verbe est vieux de nos jours et ne figure plus dans les dictionnaires usuels.

gâ (Ms. 1 Cahier A; Ms. 2); (petit) gâa, «jeune garçon». Au sens de "petit garçon, enfant", gars est bien attesté dans les parlers du Nord-Ouest et du Centre; petit gars a été relevé en Bretagne et en Normandie (FEW 17, 617b sous *wrakkjo). Richelet 1680 précise le niveau de langue : "Ce mot signifie garçon, mais il ne se dit guere à Paris et méme il ne se dit que dans le bas burlesque. Le petit gars lui vint sauter au cou." Petit gars est consigné avec la mention «régional ou vieilli» (TLF), ou "rural» (GLLF; ReyHist). Le mot gars, dans toutes ses acceptions, est généralement senti comme familier de nos jours (PRob 1993; PLar 1998). Petit gars a été recueilli lors d'enquêtes orales (PPQ 1815; Lavoie 2756); encore usuel en français québécois (DQA). Recueilli en Acadie (Massignon 1716). V. aussi Aspects phonétiques.

galipote (courir la ) (Ms. 1 Cahier A); “[courir le guilledou, la prétentaine]». Extension sémantique de courir la ganipote au sens de 
"vagabonder la nuit», relevé en Aunis et en Saintonge. Le mot est un apport des parlers de l'Ouest; galipote désigne en poitevin une «course effrénée; une course de nuit par suite de sortilège» (FEW 17, 478b sous wala). Galipote ne s'emploie au Québec que dans la locution courir la galipote; c'est pourquoi la définition que donne Viger au début de son article : galipote «bordel, boucan" ne nous semble pas exacte. Potier relève courir la galipote au Détroit en 1745 (HalPot 267). La locution est consignée dans Clapin, Dionne et Glossaire. Recueillie dans les années 1970 (PPQ 2291; Lavoie 2802), elle est encore bien vivante (DQA, «rechercher des aventures amoureuses»). Connue également en Acadie (Massignon 1774).

garde-corps (Ms. 1 Cahier A). V. Datations.

gaspiller (Ms. 1 Cahier A); «[détériorer, abîmer, mettre en mauvais état du linge, des vêtements]». Ce sens est consigné de Furetière 1690 jusqu'au XIX ${ }^{\mathrm{e}}$ s. Attesté dans les parlers normands et en wallon, «gâcher, endommagen (FEW 14, 195a sous waspa-). TLF l'enregistre avec la mention «vieilli». Figure dans Glossaire, Bélisle ${ }^{3}$ : («détériorer par malfaçon : gaspiller une chemisem).

gavache (Ms. 1 Cahier A; Ms. 2); "poltron». Attesté depuis le XVI s. au sens d' «homme lâche»; donné comme "vieilli» depuis Boiste 1829. Le mot a également été relevé en normand, en saintongeais et dans des parlers du Centre (FEW 4, 4b sous " $g a b a$ ). Consigné dans TLF qui le donne comme peu usité et vieux. L'emploi au féminin, une gavache, avec le même sens, ne semble pas avoir été relevé ailleurs.

georges (faire ses ) (Ms. 1 Cahier A; Ms. 2). V. Aspects phonétiques.

gérémium (Ms. 1 Cahier A). «[plante de la famille des géraniacées]». Désigne le Géranium Robertianum (Linné) (RollFlore III, 308; MVictFl). Les horticulteurs appellent aussi géranium le pélargonium.

Le Géranium Robertianum est aussi appelé bec-de-grue. Figure dans les dictionnaires depuis 1708 (FEW 1,309b sous beccus; RollFlore III, 309). V. également Aspects phonétiques.

ginseng (tomber comme le ) (Ms. 1 Cahier B; Ms. 2); «tomber tout-àcoup et sans espoir apparent de se relever». Fait allusion au commerce du ginseng qui tomba brusquement en raison de la mauvaise qualité du produit. Marie-Victorin qui relève l'expression, l'explique ainsi : "Malheureusement, au lieu de laisser la racine sécher lentement dans les greniers, on la séchait au four pour aller plus vite. Ce fut la cause de la dépréciation du Ginseng canadien et bientôt de ce grand trafic il ne resta que le dicton populaire : "C'est tombé, ou ça tombera comme le Ginseng!"» (MVictFl 410). L'expression a disparu. 
glumer (Ms. 1 Cahier A); «[dépouiller qqn]». Le mot n'a pas été relevé dans la documentation que nous avons consultée. Il s'agit peut-être d'une création verbale issue de glume, «enveloppe d'une graine», ou de grume, «écorce qui reste sur le bois coupé non encore équarri» (ReyHist sous glume et grume), sur le modèle de plume, plumer, ce dernier verbe signifiant également "dépouiller, voler qqn»; il est attesté dans ce sens figuré depuis le XIII' ${ }^{\mathrm{e}}$. (ReyHist).

gouette (Ms. 1 Cahier A). V. Aspects phonétiques et Aspects morphologiques.

gouliâ (Ms. 1 Cahier A; Ms. 2); subst., «personne qui mange avidement et malproprement". Attesté sous la forme goulias au XIII' s.; il signifie "glouton". Il a été relevé en Normandie, à l'île anglo-normande de Guernesey, dans le Bourbonnais et en Bourgogne (FEW 4, 318a sous gula). Figure dans Dionne et Glossaire (sous gouliat). Recueilli sporadiquement dans les années 1970 (PPQ250). Vieux de nos jours. V. aussi Aspects phonétiques.

[gouliafre] (Ms. 1 Cahier A et Ms. 2 sous gouliâ); même sens que gouliâ. D'abord relevé sous la forme gouillafre en 1630, puis sous celle de gouliafre de Furetière 1690 à 1930 (FEW 4, 318b sous gula). Bien attesté dans les parlers d'oïl sous les deux formes. Le mot, sous la graphie goulafre (depuis 1821), est encore vivant au sens de "goinfrem, dans le nord-est de la France et en Belgique (ReyHist sous goule; PRob 1993; PLar 1998).

graffigner (Ms. 1 Cahier A); «entamer et déchirer légèrement la peau avec les ongles». Grafigner, "gratter avec les ongles», est attesté au XIII" s. et depuis le $\mathrm{XV}^{\mathrm{e}}$ s. au sens d'éegratigner». Figure dans les dictionnaires, de Furetière 1690 à Larousse 1948 (cités dans FEW); Bescherelle 1858 précise : «Expression populaire que les gens sans instruction emploient quelquefois par corruption pour Égratigner.» Largement répandu dans les parlers d'oï et d'oc, ainsi qu'en francoprovençal; connu également dans certains parlers en parlant des chats qui griffent (FEW 16, 350b sous krafla). Consigné dans Dunn, Clapin et Dionne (sous graffigner), Glossaire (sous grafigner). Recueilli dans les années 1970 (PPQ 2140; Lavoie 2394) et encore bien vivant; s'emploie également pour griffer en parlant d'un chat (DQA sous grafigner). Relevé en Acadie (Massignon 1538).

graisse (Ms. 1 Cahier B); "[volée de coups]». De graisser, "battre». Attesté notamment à la fin du XVIII $\mathrm{e}$ s. dans le parler de Paris au sens de «forte réprimande» et de "volée de coups" en normand (FEW 2/2, $1282 \mathrm{~b}$ sous crassus). 
graisser (Ms. 1 Cahier B); «[rosser]». Attesté dans les parlers normands. Le verbe entre dans des expressions telles que graisser les épaules à qqn «bâtonner», qui a été relevée en français populaire depuis Furetière 1690, et graisser la peau, attestée avec la même signification depuis Féraud 1787 (FEW 2/2, 1282b sous crassus). Graisser figure dans Glossaire. V. Mècher et Moucher.

graissoux, ouse (Ms. 1 Cahier A); adj., "gras, grasse». Variante dialectale de l'adj. graisseux; graissoux est bien attesté dans les parlers du NordOuest et de l'Ouest, ainsi qu'en langue d'oc (FEW 2/2, 1277a sous crassia). Figure dans Clapin (sous graissou, oue), Dionne (sous graissou), Glossaire (sous graissoux, ouse).

En emploi substantivé, graissoux a été recueilli sporadiquement dans les années 1970 (PPQ 1912 : [gresu], "femme malpropre»; PPQ $1284 B$ et Lavoie 397, "aide cuisinier»). Le mot est probablement disparu de nos jours. V. également Aspects morphologiques.

griche-poil (Ms. 1 Cahier A); «malin, espiègle». FEW ne le relève qu'au Canada. Cf. en Anjou, griche-midi, «hargneux» (FEW 16, 393b sous *krisan). Consigné dans Clapin et Dionne. Recueilli dans les années 1970 (PPQ 1837B : «enfant colérique»). Connu également en Acadie (PoirAcad : "personne revêche»).

griche-poil (à ) (Ms. 1 Cahier B). V. Regriche ou griche-poil (à ).

gricher (Ms. 1 Cahier A); "grincer les ou des dens». Grisser les dens au $\mathrm{XIV}^{\mathrm{e}}$ s.; gricher les dentz au XVI ${ }^{\mathrm{e}}$ s. La forme gricher a été relevée notamment dans les parlers du Nord-Ouest, en Poitou et en français populaire (FEW 16, 393a sous ${ }^{*} k r i s a n$; MinPoit). Consigné dans Clapin, Dionne et Glossaire. Largement recueilli dans les années 1970 au sens de "grincer des dents" (PPQ 2106; Lavoie 2354); encore bien vivant de nos jours au Québec (DQA). Relevé en Acadie (Massignon 1478).

grichou (Ms. 1 Cahier A); «[enfant] malin». Déverbal de gricher; bien attesté sous les formes grichu, gricheux, grissou, grifoux, en picard et dans des parlers du Nord-Ouest et du Centre, où il signifie "de mauvaise humeur, grognon» (FEW 16, 393a sous *krîsan). Consigné dans Dunn, Clapin et Dionne (sous grichoux), Glossaire (sous grichou). Recueilli dans les années 1970 (PPQ1837A et Lavoie 2740 : «enfant colérique»). Relevé en Acadie (PoirAcad : «revêche, hargneux»).

Grichou «[personne] laide» est une extension du sens précédent; cf. en Bourgogne grichoux, "grisâtre, incolore (de la figure)»; cf. également en Normandie, le verbe gricher, qui signifie notamment "faire une mine désagréable; faire des grimaces» (FEW $i b$.). 
[guenille] (Ms. 1 Cahier A sous amarrer et apicbimon); «[lambeau de vieux linge, de tissus, chiffon]». Attesté sous la forme gnille en moyen français. Mot emprunté aux parlers du Centre et de l'Ouest. Attesté au sens de «chiffon» dans Cotgrave 1611; il prend, en outre, le sens de "vêtement sale et déchiré» à partir de 1664 (FEW 14, 113a sous *wadana-; GuirÉtym ; ReyHist). Le sens de «chiffon, lambeau déchiré de vêtements» figure encore dans les dictionnaires du XIXe s. (Bescherelle 1858; Littré). Encore usuel au Québec : «morceau de vieille étoffe, de vêtement dont on ne se sert plus» (DQA).

gueusaille (Ms. 1 Cahier A sous gueusasse; Ms. 2); «canaille». Dérivé de gueux; attesté depuis 1630, "groupe de mendiants"; le mot est aujourd'hui sorti d'usage (FEW 16, 98b sous guit; ReyHist sous gueux).

gueusasse (Ms. 1 Cahier A; Ms. 2); «canaille; coquin». Dérivé de gueux; variante de gueusaille. Relevé dans le parler de Paris (1790) au sens de «troupe de gueux»; la forme gueusasse est surtout attestée en langue d'oc et en franco-provençal. Gueusasse a également été relevé comme variante de gueuse (FEW 16, 98b sous guit; NisPar 134). En français québécois comme en français général, le mot est aujourd'hui sorti d'usage.

habitant, ante (Ms. 1 Cahier A; Ms. 2); «agriculteur, laboureur». Le mot désigne en français général depuis le XIII' $\mathrm{s}$. «celui qui habite un endroit, une maison"; il s'est appliqué, avec le développement des colonies, à un "particulier auquel le souverain a accordé des terres à défricher et à planter dans les colonies»; il est synonyme de colon pour Trévoux 1771 (FEW 4, 369a sous babitare; ReyHist sous babiter; JunPMeun 126-127). Par extension, babitant a pris à la fin du XVII ${ }^{\mathrm{e}} \mathrm{s}$. (1675) le sens de "personne qui cultive la terre». Potier relève le mot à Lorette en 1743-1744 (HalPot 271). C'est le mot usuel pour Viger qui l'emploie constamment dans ses articles. Consigné dans Dunn, Clapin, Dionne et Glossaire. Le mot est vieux et péjoratif de nos jours au Québec (DQA). Connu en Acadie dans les «régions d'influence canadienne" (Massignon 623). Relevé dans les parlers franco-américains de la Louisiane (DitchyLouis), du Mississippi (McDermMiss) et du Missouri (DorrSteGen), ainsi qu’à la Réunion, à la Martinique, et à Haïti (ChaudRéun I, 599).

Le féminin habitante, «celle qui habite la Campagne, qui cultive la terre» n’a pas été retenu par les glossairistes québécois (Dunn, Clapin, Dionne et Glossaire, entre autres).

[hardes] (Ms. 1 Cahier A et Ms. 2 sous butin); «[vêtements d'une personne]». Attesté au $\mathrm{XII}^{\mathrm{e}}$ s. en ancien normand sous la forme fardes au sens d'«habillements ordinaires». Attesté sous la forme bardes, 
«bagage, vêtements, linge et coffre d'une personne», depuis 1539 (FEW 3, 416a-b, sous fard; ReyHist). Le mot figure encore sans mention dans les dictionnaires du début du XXe s. (DG). Donné aujourd'hui comme vieux ou régional en français général (PRob 1993). Recueilli dans les années 1970 au Québec (PPQ 1907; Lavoie 2528). Vieux de nos jours.

herbe à dinde (Ms. 1 Cahier A); «[achillée millefeuille]». Acbillea millefolium, L. «Les noms vulgaires appliqués en France à cette plante sont innombrables, mais "Herbe à dindes" paraît être un pur canadianisme, fondé d'ailleurs sur l'emploi de la plante dans l'alimentation de cette volaille» (MVictFl 592). Consigné dans Dionne et Glossaire (sous herbe). Recueilli dans les années 1970 (PPQ 777, qui relève aussi le synonyme berbe à mille feuilles; Lavoie 3148). En Acadie cette plante se nomme berbe à dindons (Massignon 233).

herbe à la puce (Ms. 1 Cahier $\mathrm{A}$ ); «[sumac vénéneux]». Rhus radicans $\mathrm{L}$. Synonyme : Rhus toxicodendron L. (MVictFl 392-393). En France, le terme herbe à puces est attesté depuis le XVI ${ }^{\mathrm{e}}$ s. et désigne une grande variété d'espèces de plantes (FEW $9,522 \mathrm{~b}$ sous pulex). Consigné dans Dionne et Glossaire. Herbe à (la) puce est le nom usuel de cette plante au Québec (DQA sous berbe). Connu également en Acadie (Massignon 196).

houiller (Ms. 1 Cahier A; Ms. 2); «changer, troquer une chose contre une autre [surtout des chevaux]». Cette acception ne semble pas avoir été relevée ailleurs que chez Viger. Houiller est peut-être à rattacher au verbe boller, qui a signifié «courir d'une foire à l'autre», «marchander sans fin", attesté en wallon (FEW 16, 222a sous bollen). Cf. également le verbe buier (prononcé également houiller), qui signifie «crier, héler» (FEW 4, 506a-b sous bui-), et bouailler «crier haut; faire des cris pour indiquer le lieu où l'on est», connu des parlers normands, hautsmanceaux et orléanais (FEW 4, 503a sous $b u-$ ). Mot sorti d'usage.

houiller (Ms. 1 Cahier A; Ms. 2); se houiller «se gorger de vin». De l'ancien français aouller, auller; ouiller est une contraction de aouiller, de l'ancienne forme de ail (ReyHist sous ceil). Emploi métaphorique de "remplir un tonneau jusqu'à la bonde». La forme pronominale est attestée en ancien français : se aouiller, «se rassasier»; relevée en Anjou et dans le Languedoc au sens de "s'enivrer» (FEW 7, 317a-b sous oculus). Cf. également dans les parlers du Nord-Ouest et de l'Ouest le sens de «rassasier». Consigné dans Glossaire (sous bouiller et ouiller). Recueilli à Verchères dans les années 1970 (PPQ252x : ouillë, «gavé»).

Être bouillé de qqn, de qqch., wêtre las, dégoûté de qqn, de qqch.». Ce sens a été relevé en Anjou et en Vendée : ceillé de, ouillé de «las de; fati- 
gué; qui en a assez de» (FEW 7, 317b sous oculus). Le mot n'est plus employé de nos jours en français québécois.

icit (Ms. 1 Cahier A). V. Aspects phonétiques.

incendie (Ms. 1 Cahier A). V. Aspects morphologiques.

[inquilibre (être dans l' )] (Ms. 1 Cabier B sous équilibre); «indécis, irrésolu». De équilibre dans son sens général d'«égalité, équivalence». L'expression signifie que les chances de faire ou de ne pas faire quelque chose sont égales. Figure dans Dionne (sous équilibre et inquilibre). Expression disparue. V. également Aspects morphologiques.

joli-cour (s'appeler, se nommer ) (Ms. 1 Cahier A; Ms. 2); "[se retrouver le bec dans l'eau]». Par antiphrase de l'expression faire le joli coeur, "faire le galant». Cf. l'expression être joli garçon, "être dans une vilaine position» (FEW 16, 286a sous jol). S'appeler suivi d'un nom a été utilisé en français populaire dans d'autres expressions, comme par exemple : s'appeler La Roche, «être brave, etc.» (NisPar 152). S'appeler joli-ceour qui, au début du $\mathrm{XIX}^{\mathrm{e}}$ s., s'employait tous les jours selon Viger, est aujourd'hui sortie d'usage.

jouquer (se ) (Ms. 1 Cahier A; Ms. 2); «se jucher». Au XII s. joquier, «être juché (d'un oiseau)». La forme pronominale a été relevée dans les parlers du Nord-Ouest et de l'Ouest ainsi qu'en franco-provençal (FEW 16, 288a sous juk; RézVoc). Consigné dans Clapin et Glossaire. Recueilli dans les années 1970 (Massicotte IV-324; PPQ 620). Relevé en Acadie (Massignon 1583).

Au figuré, se jouquer (d'une personne) «se mettre [loger, habiter]». Relevé dans ce sens en picard dans son emploi absolu : jouquer, «loger, habiter» (FEW 16, 288b); le français populaire connait également cet emploi sous la forme jucher (ReyHist).

Jouqué «[juché, perché]». Jouquer, v. intr., est bien attesté dans les parlers d'oïl et d'oc et en franco-provençal (FEW 16, 288a; RézVoc). Relevé en Acadie (Massignon 952).

jouquoir (Ms. 1 Cahier A; Ms. 2); «juchoir». Attesté en Picardie et en Bourgogne (FEW 16, 288b sous juk). Consigné dans Dionne et Glossaire. Recueilli lors d'enquêtes orales au Québec (PPQ 621; Lavoie 1415) et en Acadie (Massignon 951).

juifrèsse (Ms. 1 Cahier A; Ms. 2); «juive». Le mot est attesté au XIVe s. sous la forme juiesse. La forme juiferesse (juivresse) a été relevée en Anjou, dans les parlers du Nord, de l'Est, en wallon et en Suisse romande (FEW 5, 53a sous judaeus; MénAnj : Juivresse). La fréquence de juifrèsse était plus grande que celle de juive au début du $\mathrm{XIX}^{\mathrm{e}}$ s., selon Viger. Le mot est aujourd'hui sorti de l'usage. 
jument (Ms. 1 Cahier A; Ms. 2); «caisse de fusils (Terme du Commerce des pelleteries)». À rapprocher du terme taureau usité dans la langue du Nord-Ouest canadien au XIX ${ }^{\mathrm{e}}$ s., au sens de «sac fait de peaux de buffle vertes, rempli de pémican ou de viande pulvérisée, pesant généralement 90 livres» (Gagnon 136).

[lèche-crachats] (Ms. 1 Cahier A sous payer); «[personne qui flatte servilement]». Crachat a été relevé comme terme d'injure pour désigner un «individu méprisable» (Édouard).

légerte (Ms. 1 Cahier A; Ms. 2). V. Aspects phonétiques.

lévier (Ms. 1 Cahier A). V. Aspects phonétiques.

lichefrite (Ms. 1 Cahier A; Ms. 2). V. Aspects phonétiques.

licher (Ms. 1 Cahier A; Ms. 2); en licher (à qqn), «[obtenir de l'argent de qqn]». Emploi figuré de lécher, «manger, boire». Le verbe a également le sens d'uobtenir qqch." : lécher un repas, "manger en parasite chez qqn" (FEW 16, 456a sous lekkon); Bescherelle 1858 relève l'expression : Lécher l'ours, "sucer les parties en prolongeant le procès». Cf. également licher, «manger son bien», relevé en Lorraine et en Suisse romande (FEW 16, 459b sous lekkon). Consigné dans Glossaire.

S'en licher les barbes, "passer la langue sur qqch.». En français général, l'expression est employée au figuré : «se dit d'un homme qui s'est vu frustrer d'un avantage attendu»; attesté dans les dictionnaires de Furetière 1690 à Larousse 1873 (FEW 16, 456a sous lekkon). Glossaire relève l'expression au sens de "se pourlécher les babines», et au figuré, «se délecter à la pensée de quelque chose de friand». V. aussi Aspects phonétiques.

[louche] (Ms. 1 Cahier A et Ms. 2 sous bicleux et vire-l'œil); "[qui est atteint de strabisme]». Au début du XIV s., louche qualifie les yeux atteints de strabisme et, à la fin du XIV $\mathrm{X}^{\mathrm{e}}$ s., une personne; ce dernier emploi a disparu (ReyHist). Littré et DG le relèvent encore sans mention. L'adjectif est encore consigné de nos jours dans ce sens, mais il est vieux (PRob 1993).

loucheur, euse, subst. et adj. (Ms. 1 Cahier A; Ms. 2). V. Datations.

mal (tomber d'un ) (Ms. 1 Cahier B); «[avoir une crise d']épilepsie».Tomber d'un mal a été relevé dans le parler populaire de Paris en 1821; attesté en wallon. Tomber de mal est attesté en normand et en haut-manceau, et tomber du mal en normand et en bourbonnais (FEW $6 / 1,126 \mathrm{~b}$ sous malus). Consigné dans Dunn ( NNous disonsTomber d'un mal») et dans Glossaire («tomber d'un mal, tomber de son mal»). Recueilli dans les années 1970 (PPQ2207 : tomber dans les mals, tom- 
ber dans un mal; Lavoie 2446 : tomber dans les mals, tomber d'un mal). Vieilli de nos jours.

Viger tire les quatre synonymes qu'il donne à ce mot du dictionnaire de l'Académie de 1798 : «On dit, Mal caduc, baut mal, pour dire, L'épilepsie. Il tombe du mal caduc, du baut mal. Le peuple dit, Mal de Saint-Jean, et plus communément, Mal de Saint, pour dire, Le baut mal». Mal caduc, mal de Saint et mal de Saint-Jean sont aujourd'hui sortis d'usage. Haut mal, qui est attesté depuis le XIV $V^{\mathrm{e}}$ s., figure dans PRob 1993 avec la mention "vieux». Le terme a été recueilli sporadiquement dans les années 1970 au Québec (PPQ 2207). Connu en Acadie (Massignon 1611).

mâle (Ms. 1 Cahier A; Ms. 2); «bonnet de laine tricottée». Il s'agit probablement d'un emploi métaphorique de mâle, «rocher, sommet rocheux», attesté en langue d'oc; cf. également le sens de «grosse tête dure» en franco-provençal (FEW 6/1,119b sous malleus; Rohlfs 68). Mâle, dans ce sens, n’a été relevé que par Viger. V. Tuque.

malin, igne (Ms. 1 Cahier A et sous rustique); «difficile, épineux, fatigant». Attesté dans c'est bien malin, «c'est bien difficile; c'est un beau miracle» depuis 1808. Malin, «difficile à faire, à comprendre; malaisé (avec une négation ou une forme équivalente)" figure avec la mention "populaire» depuis DG (FEW 6/1,107b sous malignus).

manche (Ms. 1 Cahier A; Ms. 1 Cahier A sous bouquin); manche de pipe, «[tuyau de pipe $]$ ». FEW ne donne que l'attestation canadienne (FEW $6 / 1,219$ a sous manicus). Consigné dans Dunn, Clapin, Dionne et Glossaire. Recueilli dans les années 1970 (PPQ 2072x; Lavoie 3139). Connu en Acadie (PoirAcad).

manchonnier (Ms. 1 Cahier B); «artisan qui travaille en pelleterie». Dérivé de manchon, «cylindre de tissu, de fourrure, pour protéger les mains». Ce sens a été relevé en picard, en saintongeais, à Nice (sous la forme manchounié) (FEW 6/1, 211a sous manica; MussSaint). Figure dans Dunn, Clapin, Dionne : "Manchonnier vient du fait que le fourreur fabrique des manchons; mais il fabrique aussi d'autres choses.»

manquer $(1 \mathrm{a} \sim$ belle) (Ms. 1 Cahier B); "[l'échapper belle]». Au sens d'«avoir laissé échapper une belle occasion», la locution est attestée depuis 1666, mais elle est donnée comme familière à partir du XIX ${ }^{\mathrm{e}}$ s. Au sens d'«avoir échappé à un grand danger», elle est consignée avec la mention «familière» à partir d'Académie 1718; «on dit plutôt l'avoir échappé belle», indique Académie 1935 (FEW 6/1, 141b sous mancus). La manquer belle ne figure plus dans les dictionnaires usuels. 
Manquer au sens de «faillir» suivi d'un infinitif sans préposition (dans : il a manqué périr et il a manqué mourir) a probablement été relevé par Viger en raison de la condamnation de cette construction par l'Académie. Considérée comme «fautive» également par Littré (GougÉtude 147). Les constructions manquer de + infinitif et manquer + infinitif sont encore courantes de nos jours en français général (ReyHist).

marier (Ms. 1 Cahier A; Ms. 2); «épouser». Marier, verbe transitif au sens d'eépouser", est attesté dès le XII ${ }^{\mathrm{e}}$ s. Largement attesté dans les parlers d'oïl et d'oc, ainsi qu'en franco-provençal (FEW 6/1,348b sous maritare). Relevé en français populaire (Bauche). Vit encore notamment dans les parlers du Nord (CartNord). «Au sens de "prendre pour femme», l'usage moderne a généralisé la construction réfléchie se marier avec (après 1170) aux dépens de l'ancienne construction transitive, marier qqn (1176)» (ReyHist sous mari). Viger, comme beaucoup d'autres auteurs, considère cet emploi du verbe marier comme un anglicisme. Consigné dans Dunn, Clapin (qui le classe comme anglicisme, p. XXX), Dionne, Glossaire. Bien attesté dans les années 1970 (PPQ 1887; Lavoie 2791) et encore usuel (DQA). Relevé en Acadie (Massignon 1731) et en franco-ontarien (LemVieux 1, 217).

[marinade] (Ms. 1 Cahier A sous marinage); "chose marinée, bouillie dans le vinaigre». Vient probablement de marinade au sens d'«aliments préparés pour se conserver des années entières à la mer», sens emprunté au langage maritime et consigné dans les dictionnaires, de 1840 à DG; il s'agit d'une extension de marinade au sens d' «assaisonnement dans lequel on laisse tremper les viandes et les poissons avant de les faire cuire» (FEW 6/1, 346a sous marinus). Clapin, Dionne et Glossaire enregistrent le mot au pluriel. Recueilli dans les années 1970 (PPQ 223; Lavoie 2199) et encore usité (DQA sous mariner : «Marinades : condiments faits de morceaux de fruits ou de légumes macérés dans du vinaigre mélangé avec des épices ou des aromates»). V. Marinage.

marinage (Ms. 1 Cahier A); «chose marinée, bouillie dans le vinaigre». Déverbal de mariner, "laisser tremper de la viande dans du vinaigre», attesté depuis Furetière 1690. En français général, marinage désigne la "préparation que l'on fait subir à certaines viandes destinées à être conservées", sens attesté depuis 1867 (FEW 6/1, 346a sous marinus). Consigné dans Glossaire (sous marinages). Recueilli dans les années 1970, mais beaucoup moins fréquent que marinade (PPQ 223; Lavoie 2199). Relevé en Acadie (Massignon 1339). V. Marinade. V. aussi Aspects morphologiques. 
matelat (Ms. 1 Cahier A); «trait qui se décoche avec un arc». Le mot désigne un "trait d'arbalète»; il est attesté sous la forme maturas au $\mathrm{XII}^{\mathrm{e}} \mathrm{s}$, matelat à la fin du XII ${ }^{\mathrm{e}} \mathrm{s}$., matelas $\mathrm{du} \mathrm{XIV}^{\mathrm{e}}$ au XVII $\mathrm{XI}^{\mathrm{e}}$ s. et materas, qui a donné la forme moderne matras consignée depuis 1608 . Le mot est donné comme terme historique depuis Richelet 1680 (FEW $6 / 1,463 \mathrm{~b}$ sous mataris).

matelot (Ms. 1 Cahier A); «insecte». Le mot, dans cette acception, est absent des sources consultées. Cf. Bescherelle 1858, qui le relève en ornithologie où il désigne l'shirondelle de fenêtre".

mauvaiseté (Ms. 1 Cahier B); «méchanceté». La forme malvaistié, "méchanceté, perversité de la nature humaine, volonté de faire le mal", est attestée dès le $\mathrm{XII}^{\mathrm{e}} \mathrm{s}$. La forme mauvaiseté est relevée depuis 1701. Le mot a été très largement relevé en France d'oil et d'oc, ainsi qu'en franco-provençal (FEW 6/1,97b-98a sous malifatius). Il a été banni des œuvres littéraires au XVII ${ }^{e}$ s. (ReyHist sous mauvais). Attesté sporadiquement dans les années 1970 au Québec (PPQ 414, 1548x, 2310), il est depuis sorti d'usage.

méchant (Ms. 1 Cahier A); "difficile, épineux, fatigant; [mauvais]». Méchant est attesté au sens de «mauvais» en français classique; il a été relevé dans de nombreux dialectes d'oil et d'oc (FEW 2/1, 28a sous cadere; ReyHist). Consigné dans Clapin, Dionne et Glossaire en parlant des chemins. Recueilli dans les années 1970 (PPQ1064, «chemin glaiseux"; Lavoie 158, "pente très raide», 160, "chemin impraticable»). V. Rustique.

mèche (Ms. 1 Cahier A; Ms. 2); «grande distance». Emploi figuré, issu probablement du sens de "cordon de fils de coton ou de chanvre que l'on fait brûler». Le mot est connu également en français québécois au sens de «longue période de temps» (PPQ 1730; DulDictC). Cf. en argot : et mèche, «et davantage, un peu plus» (FEW 6/3,325a sous myxa). Figure dans Glossaire et DulDictC. V. Pipe.

mèche (Ms. 1 Cahier A; Ms. 2); «extrémité la plus déliée d'un fouet». V. Datations.

mècher (Ms. 1 Cahier A; Ms. 2); «rosser». De mèche, «ficelle d'un fouet»; probablement sur le modèle de fouet, fouetter. Cf. le sens figuré de "gronder qqn" dans le Limousin (FEW 6/3, 324a sous myxa). Glossaire l'enregistre au sens de «maltraiter». V. graisser et moucher.

menoirs (Ms. 1 Cahier A); «[chacune des] deux piéces de bois qui se prolongent en avant d'une charette, ou que l'on attache par une chaîne de fer aux voitures d'hyver en Canada et entre lesquels est placé le cheval qui les traine». Le mot est attesté au XIII ${ }^{\mathrm{e}}$ s. : meneoire, «timon d'une 
voiture»; en moyen français : menueres, «les deux brancards d'une charrette». Relevé en picard, en normand, en orléanais et en bourguignon (FEW 6/2,103b sous minare). Consigné dans Dunn (sous menoir), Clapin (sous menoires), Dionne (sous ménoire) et Glossaire (sous menoire). Recueilli lors d'enquêtes orales au Québec (PPQ 1119; Lavoie 921) et en Acadie (Massignon 678). V. Travail.

[meuble] (Ms. 1 Cahier A sous casseau; Ms. 1 Cahier B sous plated); «vase, [vaisselle d'argent]». Meuble a eu le sens d' "ustensile de ménage, objet servant à garnir une maison, sans en faire partie», du XVe s. à la fin du $\mathrm{XVIII}^{\mathrm{e}}$ s. (FEW 6/3,1a sous mobilis). Furetière 1727 : «Les meubles précieux sont les tableaux, la vaisselle d'argent.» Cet emploi est critiqué à la fin du XVIII ${ }^{\mathrm{e}} \mathrm{s}$ : : «Il ne faut pas confondre meuble et ustensile : on les distingue bien dans une cuisine. [...] Un auteur moderne a confondu ces deux mots. Pourquoi, dit-il, ne pas substituer le fer au cuivre dans les meubles servant à la préparation des alimens et des remèdes. Le mot est très impropre» (Féraud 1787). Enfin, Littré restreint l'usage à «certains objets qu'on peut porter sur soi. Ce couteau à plusieurs lames est un meuble fort commode.» Sens aujourd'hui disparu en français général comme en français québécois. V. Casseau.

micmac (Ms. 1 Cahier A); «baragouin, baragouinage». En jersiais, le mot a le sens de «discours confus» et en Franche-Comté de «bavardage, racontars»; il s'agit probablement d'une extension du sens d'«intrigue; désordre, situation embrouillée» (FEW 16, 589b sous muytemaker). Une allusion à la langue des Amérindiens de ce nom n'est peut-être pas à écarter. Figure dans Dionne : «Langage incompréhensible». Micmac a désigné le parler populaire des Franco-Américains de la Nouvelle-Angleterre (DulDictC).

mistimus (en ) (Ms. 1 Cahier B; Ms. 2); «bien, très bien». Il s'agit peutêtre d'une création de l'auteur. Mistimus était l'un des pseudonymes de Viger avec Mistigri. Fait peut-être référence au valet de trèfle, qui se nomme misti ou mistigri et qui est la carte maittresse dans le jeu du même nom (FEW 6/2,178a sous mit-).

[mitaine] (Ms. 1 Cahier B sous manchonier); «[moufle fourrée de fourrure]». Mitaine au sens de "gant qui couvre complètement la main et n'a qu'une division pour le pouce» est attesté depuis le $\mathrm{XII}^{\mathrm{e}}$ s. (FEW 6/2,177a sous mit-). Moufle date du XIII ${ }^{\mathrm{e}} \mathrm{s}$. En français général, les deux mots ont été employés concurremment jusqu'à la fin du XIX ${ }^{\mathrm{e}} \mathrm{s}$. En Nouvelle-France, mitaine est attesté dès 1609 chez Lescarbot. C'est le mot usuel encore de nos jours au Québec.

moindrement (le $\sim$ ) (Ms. 1 Cahiers A et B). V. Aspects syntaxiques.

mondaine (orge ) (Ms. 1 Cahier A; Ms. 2). V. Aspects phonétiques. 
Montréaliste (Ms. 1 Cahier B). V. Aspects morphologiques.

moucher (Ms. 1 Cahier A); «rosser». Attesté au sens de «battre, infliger une correction" dès le XVI ${ }^{\mathrm{e}}$ s. Cette acception a été consignée dans les dictionnaires à partir de 1640; figure avec la mention "populaire» dans Littré. Bien attestée dans les parlers d'oil et en franco-provençal (FEW 6/3,177a sous muccare). De nos jours, le mot est usuel en français général, dans son sens figuré et familier de «remettre (qqn) vertement à sa place, lui dire son fait» (PRob 1993). Consigné dans Dunn, Clapin et Dionne. Encore vivant au Québec, où il signifie «réprimander (qqn) durement» et «recevoir une râclée» (DQA). V. Graisser et Mècher.

mouiller (Ms. 1 Cahier A; Ms. 2); «pleuvoir». «D'après l'emploi du participe passé adjectivé mouillé au sens de "pluvieux» (XIII ${ }^{\mathrm{e}}$.), il a eu en langue classique le sens de "pleuvoir", y compris à la forme impersonnelle (1636)» (ReyHist). Ce sens s'est maintenu en français populaire et dans les parlers du Nord-Ouest et de l'Ouest, en wallon et en Suisse romande (FEW 6/3, 48a sous "molliare; RézOuest et RézVendée). Potier a relevé le mot au Détroit en 1744 (HalPot 280). Dunn, qui l'enregistre, écrit que c'est ale plus canadien des mots». Courant au Québec dans la langue familière (DQA). Relevé en Acadie (Massignon 77) et dans les parlers franco-américains de la Louisiane (ReadLouis) et du Missouri (DorrSteGen).

mulon (Ms. 1 Cahier A); «grosse meule [de foin]». D'abord attesté sous la forme muilun, muillon au $\mathrm{XII}^{\mathrm{e}}$ s., puis mulon au $\mathrm{XIII}^{\mathrm{e}}$ s. Mulon, qui désigne soit une "petite meule», soit une "grosse meule de foin», est très répandu dans les parlers d'oil ainsi qu'en Suisse romande; le sens de "grosse meule» est attesté en Bretagne romane et en Suisse notamment (FEW 6/3, 307b-308a sous mutulus). Recueilli lors d'enquêtes orales au Québec (Massicotte III-177; PPQ 814B et 835) et en Acadie (Massignon 703 et 712). V. Veuilloche.

naturel (Ms. 1 Cahier A; Ms. 2); «qui contribue à la santé». Dès le XII ${ }^{\mathrm{e}}$ s., le mot est attesté au sens de "produit par la nature seule, sans que l'homme s'en mêle» (FEW 7, 50a sous naturalis; ReyHist). Il s'agit d'un glissement de sens qui figure dans Glossaire : "Travailler comme ça, c'est pas naturel.»

nijon (Ms. 1 Cahier A; Ms. 2); «bout d'homme». Nijon dans ce sens ne semble pas avoir été relevé ailleurs que chez Viger. Sous la forme nigeon, le terme a désigné en normand un unigaud, une personne minutieuse»; dans le Maine, en Anjou, en Orléanais et en Touraine, il a signifié «qui passe son temps à des riens» (FEW 7, 118a sous ${ }^{*}$ nidicare). Cf. également, de nos jours, dans les parlers de l'Ouest : nijhon, 
"gamin dissipé et vicieux" (DubGloss). Le mot est aujourd'hui sorti d'usage au Québec. V. Bougon.

niveleux (Ms. 1 Cahier A); «qui requiert beaucoup d'application d'esprit, de tems, et de dextérité». Nivelleux a vécu en français général au sens de "qui ne s'amuse qu'à des bagatelles», d'Académie 1694 à Trévoux 1771. De niveler, «vétiller, s'amuser à des bagatelles», attesté de 1663 à Trévoux 1771, et «mettre beaucoup de temps à faire peu de chose», relevé en Champagne; cf. en normand le substantif nivelerie, «travail minutieux consacré à des bagatelles» (FEW 5, 295a sous libella; ReyHist sous niveau). Consigné dans Dionne et Glossaire. Niveleux semble sorti de l'usage au Québec; cependant, un verbe de la même famille, nivelasser, "faire de menues besognes» (PPQ278; Lavoie 810 et 2048), et un nom, nivelassage (PPQ278s), ont été recueillis dans les années 1970 .

nix (Ms. 1 Cahier A); «[non, pas du tout]». Emprunté à l'allemand nichts. Mot en usage au XIX ${ }^{\mathrm{e}}$ s. dans l'argot de Paris; largement relevé dans les parlers d'oil, ainsi qu'en Suisse romande (FEW 16, 599b sous nichts). Le mot est probablement entré en français canadien par l'anglais; en effet, nix est également connu en anglais britannique et américain dans la langue argotique depuis 1789 (OED, Webster). Consigné dans Dionne (sous niqse) et Glossaire. Ce mot n'est plus usité au Québec.

L'expression nix for stein ne figure pas dans les sources consultées.

notureau (Ms. 1 Cahier A); «jeune cochon que l'on engraisse pour tuer». De nurture au $\mathrm{XI}^{\mathrm{e}} \mathrm{s}$., nourreture, norreture au $\mathrm{XIV}^{\mathrm{e}} \mathrm{s}$, au sens de «bétail qu'on élève». Le mot, surtout attesté en Normandie, désigne un «jeune porc sevré et qu'on nourrit avec soin»; relevé sous les formes norreturiau, norturiau, notureau, nourturiau (FEW 7, 253b sous nutritura). Consigné dans Glossaire (sous nortureau et nourritureau). Bien attesté dans les documents des XVII et XVIII s. (JunPron 21, 163, 234), mais n'est plus connu aujourd'hui; recueilli sporadiquement dans les années 1970 (Lavoie 1351). V. Porchais. V. également Aspects phonétiques.

office (Ms. 1 Cahier A;Ms. 2). Viger a tiré du dictionnaire de l'Académie, les sens français du mot office. Rendre de bons offices, attesté depuis le $\mathrm{XVI}^{\mathrm{e}}$ s., subsiste, spécialement dans le domaine des relations internationales (ReyHist sous office ${ }^{\top}$ ). Le sens de «devoir» dans c'est l'office d'un bon père est donné avec la mention "vieux» dans (PRob 1993); celui de «fonction que l'on doit remplir» dans $j$ 'ai tel office dans l'administration est également vieilli (PRob 1993). Enfin, office au sens de «lieu où l'on garde la vaisselle, etc.», qui était du genre féminin à 
l'époque de Viger, est aujourd'hui employé au masculin (ReyHist sous office ${ }^{2} ;$ PRob 1993). V. Emprunts à l'anglais.

oiseau blanc (Ms. 1 Cahier A); «ortolan». Plectrophane des neiges ou bruant des neiges, Plectropbenax nivalis $\mathrm{L}$. (GodOis). Consigné dans Dionne («bruant, excellent à manger»), Bélisle ${ }^{3}$ («nom pop. du plectrophane ou bruant des neiges, sorte d'ortolan des pays nordiques»). Recueilli dans les années 1970; oiseau blanc est l'appellation populaire et usuelle de cet oiseau au Québec (PPQ 1547; Lavoie 719). Connu en Acadie (PoirAcad sous oseau, oueseau). V. Ortolan.

orignal (Ms. 1 Cahier A; Ms. 2); «élan». Désigne l'Alces alces americana, l'élan d'Amérique. Le mot est d'origine basque; la forme primitive orignac est la forme du pluriel de oregna "cerf" (FEW 20, 18b sous orein). La forme orignac est attestée dès 1603 dans Champlain : «Il s'en alloit à Tadousac pour troquer des flesches, \& chairs d'orignaos (Champlain I, 49). Boucher, qui en 1664, fait une bonne description de l'animal, le nomme original (BouchHist 54). La même année, la forme orignal est attestée dans un document du Conseil souverain (cité dans Massignon 362). Le mot est consigné dans les dictionnaires depuis Trévoux 1704. C'est le terme usuel encore de nos jours pour désigner cet animal au Québec (DQA) et en Acadie (Massignon ib.).

[ortolan] (Ms. 1 Cahier A sous oiseau blanc); autre nom de l'oiseau blanc (v. ce mot). Consigné dans Bélisle ${ }^{3}$. Relevé dans les années 1970 (PPQ 1547). Le mot désigne aussi et plus généralement l'Eremophila alpestris, l' «alouette commune» et l'«alouette cornue», sens que relèvent Dionne, Glossaire, PPQ1531, RoussBouch 322 et Massignon 400. V. Oiseau blanc.

ouète (Ms. 1 Cahier A). V. Aspects phonétiques.

papier-nouvelle (Ms. 1 Cahier A); «papier-public». Attesté, au pluriel, dans les dictionnaires, de Féraud 1788 à Académie 1878 (FEW 7, 591a sous papyrus). Dunn, qui enregistre le mot, le considère comme un anglicisme (sous papier-nouvelles : «Trad. de Newspaper, mais traduction baroque et inutile, car Journal dit la même chose»).

Papier-public est attesté de 1760 à Académie 1878 (FEW ib.). Relevé notamment au pluriel et sans mention dans Littré et Académie 1878. DG considère le mot comme vieilli.

paré, ée (Ms. 1 Cahier A; Ms. 2); "prêt, te». Attesté au XIVe s. au sens de "préparé»; connu comme terme de marine, paré, "prêt à faire quelque manœuvre ou à se battre», depuis 1702 . Attesté au sens général de "prêt", en argot et dans les parlers picards, bretons, poitevins et saintongeais (FEW 7,622b-623b sous parare). Recueilli dans les années 
1970 (PPQ 277), mais vieilli de nos jours au Québec. Relevé en Acadie (Massignon 1233), en franco-ontarien (LemVieux 2, 31), en Louisiane (ReadLouis) et à la Réunion (ChaudRéun II, 828).

passe-rose (Ms. 1 Cahier B); «rose d'outremer, ou rose trémière». Althaa rosea. Le mot est connu depuis le XIII ${ }^{\mathrm{e}} \mathrm{s}$. Il a été relevé notamment en Bretagne romane ainsi qu'en wallon (FEW 10, 479b sous rosa; RollFlore III, 83). Donné comme synonyme régional de rose trémière (TLF; PRob 1993). Très largement attesté dans les années $1970 \mathrm{au}$ Québec (PPQ1683; Lavoie 336) et encore vivant.

Rose d'outremer, autre nom que Viger donne comme synonyme, est attesté dès 1500 et figure dans les dictionnaires jusqu'à DG (FEW 14, 11a sous ultra). Le mot a donné par altération rose trémière (PRob 1993). L'appellation mauve de jardin a été relevée en 1767 (RollFlore ib.).

[peinturer] (Ms. 1 Cahier A sous cariole); «[couvrir de couleur]». Dérivé de peinture. Au sens d'wenduire qqch. d'une seule couleur", peinturer est attesté depuis le $\mathrm{XV}^{\mathrm{e}} \mathrm{s}$., mais il est donné comme "peu usité» de 1784 à Académie 1878 (FEW 8, 430b sous pictura). En français général, le mot a pris le sens péjoratif de «faire de la mauvaise peinture» (ReyHist sous peintre); il est cependant encore utilisé dans la langue technique (TLF). Le mot est vieux et il est consigné avec la mention «régionalisme canadien» dans TLF. Certains grammairiens déplorent l'abandon, en français général, de la forme peinturer : «Combien de personnes oublient ou ignorent que peinturer existe à côté de peindre, avec un sens très différent, et qu'il vaudrait beaucoup mieux dire peinturer que peindre une grille ou une porte cochère!» (Stapfer 191). Peinturer est encore courant au Québec pour décrire l'action de «couvrir de peinture un mur, un meuble, etc.» (DQA).

pelleter (Ms. 1 Cahier B). V. Datations.

pelote (Ms. 1 Cahier A sous pelote et retontir); «[balle à jouer]; balle du jeu de paume». Le mot désigne au XII ${ }^{\mathrm{e}}$ s. une «boule de métal» et la «balle du jeu de paume avant qu'elle ne soit recouverte de drap" attesté depuis 1723 (FEW 8, 480a sous pila). Pelote a été relevé notamment en Normandie et en Saintonge au sens général de «balle à jouer» (FEW ib.).

Jeu de pelote a été relevé en français général (Encyclopédie sous paume).

Pelote de neige, «boule de neige», est attesté de 1587 à Larousse 1923 (cité dans FEW); Académie 1935 recommande d'employer plutôt boule (FEW 8, 480b sous pila). Pelote de neige a disparu du français général (ReyHist). Au Québec, lors d'enquêtes orales dans les années 
1970 , boule et motte de neige étaient plus attestés que pelote (PPQ 1214; Lavoie 125, 126). De nos jours, les mots usuels sont balle, boule et motte de neige (DQA).

Les termes battoir et raquette, que Viger donne dans son article pelote, sont des termes du jeu de paume, employés dans leur sens technique. Le battoir est "un instrument rond ou quarré par un bout, garni d'un long manche, le tout couvert d'un parchemin fort dur : on s'en sert à la longue paume pour chasser les balles" (Encyclopédie). La raquette est «un instrument propre à jouer à la courte paume ou au volant. C'est une palette faite ordinairement d'un treillis de cordes de boyaux de chat, fort tendue \& montée sur un tour de bois qui a un manche de médiocre longueur» (ib.).

perdrix (Ms. 1 Cahier B); "faisan sauvage». Tétraonidés. Le mot est attesté depuis le XII ${ }^{\mathrm{e}}$ s.; il désigne en France la Perdix cinerea (FEW 8, 226a sous perdix; RollFaune II, 335). Au Canada, c'est un générique qui s'applique aux différents genres et espèces de la famille des Tétraonidés (GodOis 126). Boucher emploie le mot en 1664 : «Il y a trois sortes de perdrix» (BouchHist 70-71 et RoussBouch 320). Relevé par Potier : "perdrix de Savannes (ou) aux yeux rouges» (HalPot 285). Nom courant encore de nos jours (PPQ 1495, «gélinotte du Canada», 1496, «tétras des savanes»; Lavoie 705 : perdrix blanche, brune, d'biver, de bois, de savane, grise, noire; DQA). Relevé en Acadie (Massignon 416, «gélinotte», 418, «tétras du Canada»).

pesâ (Ms. 1 Cahier A); «tige [sèche] des pois». Sous la forme pesaz au sens de «chaume de pois», le mot est attesté depuis le $\mathrm{XII}^{\mathrm{e}}$ s. Il est connu de la majorité des parlers d'oil (FEW 8, 608a sous pisum). Consigné dans quelques dictionnaires sous la forme pesat (Bescherelle 1858; Littré; DG). Potier le relève à Lorette en 1743-1744 : pésa (HalPot 285). Recueilli dans les années 1970 (Massicotte III-279; PPQ 899; Lavoie 1167); relevé également en Acadie (Massignon 782). V. aussi Aspects phonétiques.

pesant (Ms. 1 Cahier B); "sorte d'oppression ou d'étouffement qui survient quelquefois durant le sommeil, en sorte qu'on croit avoir un poids sur l'estomac». Substantivation de l'adjectif pesant, «indisposé», attesté au XIII ${ }^{\mathrm{e}}$ s.; cf. également en moyen français et en français moderne le sens d'«alourdi par un mal (du corps, d'une partie du corps)", de Furetière 1690 à Trévoux 1771 (FEW 8, 190b sous pensare). Cf., en français du XVI ${ }^{\mathrm{e}}$ s., pesard (1546) et pesart (1566) au sens de "cauchemar» (FEW 8, 192a). Figure dans Clapin (sous pésant), Dionne et Glossaire. Recueilli lors d'enquêtes orales au Québec (PPQ 2173; LorBeauce) et en Acadie (Massignon 1594). Le mot est aujourd'hui sorti d'usage. 
peter (Ms. 1 Cahier A; Ms. 2); «gercer [mains, lèvres]». Attesté en Bretagne romane, en Saintonge, dans le Limousin et en Aquitaine (FEW 8, 133a-b sous peditum). Consigné dans Glossaire.

$P e t e r$ «déchirer par quelque effort» a été relevé en langue d'oc (FEW ib.).

Peter «crevasser [de la terre]»; au sens général de «craquer», péter a été largement relevé en France d'oïl et d'oc (FEW ib.).

Les autres sens de péter que Viger relève sont français.

peter (Ms. 1 Cahier A ); «mourir». V. Datations.

pincettes (baiser en ) (Ms. 1 Cahier B sous baiser); "prendre doucement les deux joues de celui qu'on baise». Attesté sous la forme baiser à la pincette dès 1640 . Baiser en pincettes est consigné dans les dictionnaires, d'Académie 1798 à Larousse 1874 (cités dans FEW); le syntagme, sous la forme baiser à pincettes, est connu également des parlers du Nord, ainsi qu'en wallon et en Suisse romande (FEW 8, 543b sous *pints-). L'expression n'est plus enregistrée dans les dictionnaires du $\mathrm{XX}^{\mathrm{e}} \mathrm{s}$. Consigné dans Dionne (sous pincette (à la) : «S'embrasser à la pincetten) et Glossaire (sous pincette : «bec en pincetten). De nos jours, l'expression usuelle est : donner un bec à (en) pincette(s) (PPQ 1831; Lavoie 2737; DQA).

pipe (Ms. 1 Cahier A; Ms. 2); «espéce de mesure de distance chez nos habitans : c'est à peu-près trois lieues»; [par extension] «grande distance». Relevé en 1803 : «Ayant plusieurs fois questionné des Canadiens de frontière sur des distances de lieux, et de temps, sur des mesures de grandeur ou de capacité, $j$ 'ai trouvé qu'en général ils n'avaient pas d'idées nettes et précises [...]. "Il y a, me disaient-ils, d'ici à tel endroit, la distance d'une ou deux fumées de pipe [...]" (Volney 416). Cette acception appartient, à l'origine, au langage des "voyageurs». Clapin qui relève le mot l'explique ainsi : "In the language of the old French "voyageurs", a pipe meant two leagues, i. e. the time of smoking a pipe. Still in use, among the French-Canadians, to mean a good distance" ClapAmer). Encore connu dans les années 1970 (PPQ 1088 : «Trajet qui dure un certain temps, le temps de fumer une pipée»). DulDictC : "Ancienne mesure de distance des coureurs de bois (quatre à six kilomètres)»; "très longue distance". Connu en franco-américain du Mississippi (McDermMiss). V. Mèche.

pistolage (Ms. 1 Cahier A); «sorte de limaçon du genre de la porcelaine». Le mot est absent des sources consultées. V. Pucelage.

[place] (Ms. 1 Cahier A sous écrapoutir); «[plancher, espace libre d'une pièce]». Dans cette acception, le mot a été largement relevé dans les parlers du Nord-Ouest, de l'Ouest et du Centre, surtout au sens de 
«plancher, sol d'une pièce» (FEW 9, 37b-38a sous platea). Consigné dans Glossaire. Ce sens est vieilli de nos jours en français québécois. Relevé en Acadie (Massignon 1104) et en Louisiane (DitchyLouis).

plairie (Ms. 1 Cahier A). V. Aspects phonétiques.

plaisant (Ms. 1 Cahier A; Ms. 2); «beau, agréable». Le mot est attesté depuis le $\mathrm{XII}^{\mathrm{e}}$ s. au sens de «qui est agréable, qui plaît» (FEW 9, 2b sous placere). Académie 1694 à 1878 précise : «Il est peu usité en ce sens, et ne s'emploie que dans des phrases négatives." Académie 1694 : «On dit en poésie, plaisant séjour, plaisante demeure, plaisant bocage.» Pour Féraud 1787, le mot est un anglicisme. Au XIX ${ }^{e}$ s., dans cette acception, plaisant est donné comme vieilli dans Bescherelle 1858, qui précise : "Il s'employait autrefois dans le sens absolu d'agréable, et il s'appliquait aux personnes et aux choses.» Plaisant est d'un usage plus courant, aujourd'hui, en français général : «Le mot, en ce sens, a vieilli; cependant, plus expressif que agréable (lié à plaire, plaisir), il retrouve un usage, notamment dans le style soutenu» (GRob). Ce qui distingue l'usage du mot en français général d'avec celui qui prévaut en français québécois, c'est d'une part la fréquence, beaucoup plus élevée en français québécois, et d'autre part le registre d'emploi : alors que le mot relève du style soutenu en français général, il fait partie de la langue courante en français québécois. Donné comme un anglicisme par Viger. Le mot anglais pleasant explique peut-être la grande fréquence de plaisant au Québec.

[poche] (Ms. 1 Cahier B sous pochetée); "[grand sac de toile dont on se sert pour mettre le blé, l'avoine, etc.]». Attesté depuis le XIV e s.; relevé dans les parlers du Nord-Ouest et du Centre (FEW 16, 638a-b sous *pokka). Encore usuel dans les parlers tourangeaux (SimTour). Bescherelle 1858 le consigne comme terme de commerce : "grand sac de toile dans lequel on met du blé, de l'avoine, etc. [...]. On dit plus ordinairement Sac.» Relevé dans Clapin. Recueilli dans les années 1970 (Massicotte III-243; PPQ 873); courant encore de nos jours (DQA : «grand sac (de papier, de plastique, de jute...) dans lequel on met des objets à transporter»).

pochetée (Ms. 1 Cahier B); «contenu d'une poche». De pocheter. Attesté une première fois avant 1608, puis repris en 1906 (ReyHist sous poche). Le mot a été relevé en normand et dans les parlers du Centre (FEW 16, 638b sous * pokka); il vit encore de nos jours en Touraine (SimTour). Les dictionnaires qui le relèvent encore le donnent avec la mention «vieux ou régional» dans ce sens (TLF; PRob 1993; ReyHist sous poche). Potier l'enregistre en 1743-1744 à Lorette (HalPot 186). Recueilli dans les années 1970 et encore vivant de nos jours (Massicotte III-244; PPQ 873; DQA sous poche). 
poisson blanc (Ms. 1 Cahier B); «[corégone]». Désigne le Coregonus clupeaformis (MélPoiss 136). En français général, poisson blanc s'applique aux poissons d'eau douce à chair pâle; se dit de certains Cyprinidés (GLLF; GRob; FEW 8, 584a sous piscis). Boucher emploie le mot en 1664 (BouchHist 79; RoussBouch 333). Encore usuel au Québec (Lavoie 773; DulDictC; DFP).

poisson doré (Ms. 1 Cahier B); «[doré]». Ce poisson existe sous deux espèces au Québec : le doré noir, Stizostedion canadense, et le doré jaune ou doré blanc, Stizostedion vitreum (RoussBouch 332). Poisson doré est attesté dès 1664 dans Boucher (BouchHist 79). Après en avoir fait une description détaillée, Kalm ajoute que la chair de ce poisson est blanche et excellente et qu'il se trouve en abondance dans le SaintLaurent, dans la région de Québec et dans celle de Montréal (Kalm 509-511). Ce poisson s'appelle de nos jours doré (DQA).

porceline (Ms. 1 Cahier A). V. Aspects phonétiques.

porchais (Ms. 1 Cahier A); "petit cochon mort, de quatre mois ou environ, que l'on a engraissé pour tuer». Porchet, "petit porc», est attesté en ancien et en moyen français; la forme porchet est bien attestée en franco-provençal; le mot prend la forme porquet (Cotgrave 1611) en langue d'oc (FEW 9, 190a sous porcus). Consigné dans Dionne (sous porchette), Glossaire (sous porchet et porchet'). Recueilli sporadiquement dans les années 1970; le mot porcelet était beaucoup plus fréquent que porchais (PPQ542A). V. Notureau.

port-épic, porc-épic (Ms. 1 Cahier A); «animal qui, comme le porc-épic, porte des piquans au lieu de poil». En France, porc-épic désigne un mammifere rongeur de la famille des Hystricidés. Le hérisson, Erinaceus europaeus, porte aussi ce nom en Normandie (FEW 9, 191a sous porcus). Le terme porc-épic désigne au Canada l'Erethizon dorsatum et il est attesté depuis 1603 (Champlain I, 11 : porc-espic). Relevé en Acadie (Massignon 388). V. également Aspects phonétiques.

L'urson est l'autre nom de l'Erethizon (Bescherelle 1858). «Buffon l'appelle "LUrson du Nord de l'Amérique ou Porc-Épic de la Baie de Hudson, Brisson"” (Massignon 388). Le "vrai porc-épic», pour reprendre les termes de Viger, est un «mammifere rongeur (hystricidé) de l'Ancien monde, à longs piquants dorsaux»; le hérisson est le nom que l'on donne à un «mammifere insectivore d'Eurasie tempérée, caractérisé par ses piquants dorsaux»; et le coendou est un «rongeur arboricole d'Amérique du Sud» également couvert de piquants (GLar).

poudine (Ms. 1 Cahier A; Ms. 2); "mêts anglois du nom de pouding». Poudine vient probablement de l'anglais pudding; le remplacement de gn par $n$ était fréquent anciennement en français québécois dans les 
mots empruntés à l'anglais (GendrPhon 43). Cette prononciation semble disparue aujourd'hui. Le mot désigne une grande variété de desserts : le «plum-pudding», un «dessert à base de pâte déposée sur des fruits», une "crème à base de lait, d'œufs ou de riz, etc.» (DQA pouding ou pudding). V. Poutine. V. également Aspects morphologiques.

poudrer (Ms. 1 Cahier A); «[tourbillonner dans le vent (de la neige)]». Extension sémantique du sens de «dégager de la poussière (en parlant de chemins, etc.)», attesté depuis l'ancien français et relevé dans ce sens dans de nombreux parlers d'oil (FEW 9, 562a-b sous pulvis). Relevé par Potier à Québec en 1743 (HalPot 289). Toujours vivant au Québec (DQA). Relevé en Acadie (Massignon 111).

poudrerie (Ms. 1 Cahier A); «neige soulevée par un gros vent». Le mot sous la forme pouldrerie a été relevé au sens de "poussière» au $\mathrm{XV}^{\mathrm{e}} \mathrm{s}$. (FEW 9, 563a sous pulvis; ReyHist sous poudre). Repris, par analogie, en parlant de la neige, dès 1695 en Nouvelle-France (CanBAloi). Relevé par Potier (HalPot 290) et par la plupart des glossairistes; Dunn ("Le mot est pur franco-canadien, et c'est le chef-d'œuvre de notre langue»), Clapin ("Le mot poudrerie serait ainsi comme synonyme de poussière de neigen). Courant encore de nos jours (DQA sous poudre : "neige fine et sèche déjà au sol et que le vent soulève en rafales, en tourbillons»). Relevé en Acadie (Massignon 110).

pourceline (Ms. 1 Cahier A). V. Aspects phonétiques.

poutine (Ms. 1 Cahier A; Ms. 2); "mêts anglois du nom de pouding». Poutine est probablement, comme l'a démontré P. Gardette, un mot issu du latin puls, pultis, «bouillie», très largement attesté, sous différentes formes, en France d'oil et d'oc (FEW 9, 549b-550a et b; Gardette 94). Le mot poutine est généralement considéré par les glossairistes québécois comme un anglicisme; considéré ainsi par Viger, Dunn, Clapin et Glossaire notamment. En français québécois, le mot désigne un «mets sucré à base de fruits et de pâte» ou une "crème à base de lait», sens donnés aujourd'hui avec la mention «vieilli ou familier» (DQA sous pouding ou pudding); poutine désigne plus généralement, de nos jours, une «portion de frites garnie de fromage en grains et recouverte d'une sauce brunâtre légèrement épicée" (DQA sous poutine). En Acadie, poutine désigne un «mets fait avec des pommes de terre râpées" (Massignon 1342 : le terme "poutine", généralement rapproché de l'anglais pudding, semble avoir une autre origine, peutêtre liée à des termes signifiant «purée»). V. Poudine.

prouvable (Ms. 1 Cahier A; Ms. 2); «facile à prouver». D'abord attesté sous la forme provable au XIV ${ }^{e}$ s., puis sous celle de prouvable au sens de «qu'on peut prouver». Relevé dans quelques parlers d'oïl, dont ceux 
du Centre et de l'Ouest (FEW 9, 404b sous probare). Le mot est absent des dictionnaires jusqu'à la fin du XVIII ${ }^{\mathrm{e}} \mathrm{s}$. C'est la raison pour laquelle Viger, ne l'ayant pas trouvé dans les sources qu'il a consultées, dit : «Il n'est pas françois.» Figure dans Féraud 1787, qui semble le considérer comme un mot nouveau : "C'est un mot de Leibnitz : il peut être utile et il est à souhaiter que l'usage l'adopte.»

pucelage (Ms. 1 Cahier A); «sorte de limaçon du genre de la porcelaine». Le mot, qui désigne le Meretrix chione, est consigné depuis Trévoux 1752 , mais ne figure plus de nos jours dans les dicionnaires usuels (FEW 9, 525b sous ${ }^{*}$ pullicella; RollFaune XIII, 15). Il est encore connu en Normandie (LepVoc 32, qui explique ainsi le mot : «allusion à la fente que présente ce coquillage. Le choix du terme a pu aussi être influencé par l'appellation porcelaine pucen). V. Pistolage.

Québecquois ou Québéquois (Ms. 1 Cahier A); «gens de Québec». Du toponyme Québec. La présence du deuxième $q$ est probablement due à la forme Quebecq donnée dans les premières attestations du toponyme qui figurent sur la carte de Levasseur dressée en 1601 et sur celle de Champlain tracée en 1613 (BlaisTop 11). Les formes Québecquois et Québequois ont eu cours aux XVIII et $\mathrm{XIX}^{\mathrm{e}}$ s. La forme actuelle Québécois date de 1817 (DugGent 133). Le mot s'applique d'abord aux habitants de la ville de Québec; il désigne, en outre, les habitants du Québec depuis les années 1960 (DugGent 133).

quêter (Ms. 1 Cahier A; Ms. 2); «mendier, quémander». Extension sémantique de quêter (d'abord sous la forme quester), «demander, recueillir (de l'argent) pour des œuvres charitables ou pieuses", attesté depuis 1588; le sens de «mendier» a été relevé en Bretagne romane et en Bourgogne (FEW 2/2,1409a sous quaerere). «De nos jours, quêter se dit pour «demander et recueillir des aumônes» [...] spécialement à propos d'une quête religieuse» (ReyHist sous quête). Recueilli au Québec dans les années 1970 (PPQ 1757; Lavoie 1785) et encore courant.

quêteur, euse (Ms. 1 Cahier A; Ms. 2); «mendiant, quémandeur». Attesté depuis le $\mathrm{XV}^{\mathrm{e}} \mathrm{s}$. au sens de «celui, celle qui quête pour des ouvres charitables ou pieuses»; le sens de «mendiant» a été relevé en jersiais sous la forme queûteux et en languedocien (FEW 2/2,1409b sous quaerere). Consigné dans Dunn, Clapin, Dionne et Glossaire (sous quêteux). Recueilli dans les années 1970 également sous la forme quêteux (PPQ 1758). Figure dans DQA (sous quête : «Anciennt. Personne qui vivait de la charité publique en quêtant de porte en porte»). C'est par souci de correction que Viger écrit quêteur au lieu de quêteux, qui est la forme usuelle de ce mot dans cette acception. Au sujet du suffixe -eur à la place de celui de -eux, v. bourasseur et fignoleur. 
quitte (avoir plus de ) (Ms. 1 Cahier A; Ms. 2); «acquet». Avoir plus de quitte à, "plus d'avantages», est un apport des parlers saintongeais (FEW 2/2, 1473a sous quietus). Consigné dans Dionne et Glossaire. Connu en Acadie (PoirAcad). V. Acquet.

quitter (Ms. 1 Cahier A); «laisser». Le sens de «laisser» est attesté dans le domaine d'oil, notamment dans les parlers picards, normands, saintongeais et bourguignons; également répandu dans le domaine d'oc et en franco-provençal (FEW 2/2,1474a sous quietus). Encore en usage dans les parlers de l'Ouest, entre autres (DubGloss). Potier le relève à Québec en 1743 : «quitte La cette Roche \{laisse La cette pierre» (HalPot 291). Recueilli sporadiquement dans les années 1970 (PPQ $527 \mathrm{x}$ : quitter avec sa mère; 529 : quitter tarir; $851 \mathrm{x}$ : quitter javeler l'avoine; $2248 \mathrm{x}$ : quitte-le faire).

racérer (Ms. 1 Cahier A); «mettre de l'acier avec du fer, afin de rendre celui-ci propre à couper». Employé pour acérer, qui est lui-même un mot rare de nos jours (PRob 1993; PLar 1998). Attesté sous la forme racherer, "regarnir d'acier", aux $\mathrm{XIV}^{\mathrm{e}}$ et $\mathrm{XV}^{\mathrm{e}}$ s. dans les parlers du Nord; il a été relevé en Normandie (FEW 24, 105a sous aciarium). Figure dans Potier : «reacerer un hache LLa retremper y remettre de Lacier» (HalPot 293). Consigné dans Glossaire. Recueilli dans les années 1970 (Lavoie 441).

ramancher (Ms. 1 Cahier A; Ms. 2); «conter, ou raconter avec diffusion [tenir des propos peu clairs]». Cette acception a été relevée en Bretagne romane, en Anjou, en Touraine, en Poitou et en Saintonge (FEW 6/1, 224b sous manicus). Potier relève ce sens au Détroit en 1748 : «je ne sçais ce qui me remmancha \{me dit» (HalPot 293). Figure dans Dionne et Glossaire. Le mot a été recueilli dans les années 1970 (PPQ 2244 : ramancher (des histoires)) et est encore vivant (DQA : «Dire, raconter (des choses insensées)»). Relevé en Acadie (Massignon 1786, «radoter»).

ramancher (Ms. 1 Cahier A; Ms. 2); «raccommoder, réparer». Ramancher a eu cours en français, du $X V \mathrm{I}^{\mathrm{e}}$ au $X V \mathrm{XI}^{\mathrm{e}}$ s., au sens de «mettre un autre manche (à un outil)»; au sens large et général de "réparer qqch.», le verbe est connu au XVI ${ }^{\mathrm{s}}$ s., «remettre en état», et a été relevé dans les parlers bas-manceaux, "réparer, refaire" (FEW 6/1, 222b sous manicus). Cette acception est encore courante au Québec (DQA).

ramasse (Ms. 1 Cahier A); «volée de coups». Relevé en Normandie et très bien attesté dans les parlers de l'Est ainsi qu'en wallon (FEW 10, 44a sous ramus). Figure dans Clapin, Dionne et Glossaire. Recueilli lors d'enquêtes orales au Québec (PPQ 1845; Lavoie 2923) ainsi qu'en Acadie (Massignon 1805). 
rapiester (Ms. 1 Cahier B; Ms. 2); «remettre des pièces à un habit ou à du linge». Viger avait d'abord écrit rapiéceter; qu'il a corrigé pour rapiester, une forme qui se rapproche d'avantage de la prononciation. Rapiéceter au sens de «mettre beaucoup de petites pièces à un habit, etc. pour le raccommoder», est attesté depuis 1624 . Cette forme entre en concurrence avec rapiécer qui est attesté depuis 1549. Richelet 1680 précise : «On devroit dire rapiécer, mais l'usage est pour rapiécettern; en 1732, sous rapiéceter, il ajoute : «L'Académie reçoit l'un \& l'autre de ces termes. Mais Danet est pour rapiécer.» Trévoux 1771 accepte les deux termes : «On dit également bien rapiécer \& rapiéceter un habit ou du linge.» Donné avec la mention "vieilli» dans DG. Largement attesté en langue d'oïl, notamment dans les parlers normands, angevins, bourguignons et champenois (FEW 8, 336a sous pettia). Consigné dans Dionne (sous rapièceter). Recueilli dans les années 1970 (PPQ 323; Lavoie 1724); relevé en Acadie (Massignon 1258). V. également Aspects morphologiques.

ratapiat (Ms. 1 Cahier A); «baragouin, ou langage qu'on n'entend point». Cf. dans le Beaujolais : faire des rapiapia, «faire des papotages" (VurpBeauj); dans l'Orléanais : ratapiole, "personne ou chose méprisable, ridicule» (MartVend); et en Anjou : ratapans, «bavardages, explications alambiquées» (VerrAnj). Consigné dans Dionne (sous ratapias) et Glossaire (sous ratapia). Semble avoir disparu.

rebrousse ou brousse-poil (à ) (Ms. 1 Cahier A); «de mauvaise humeur». Relevé en Anjou, être à rebous-poil, «être de mauvaise humeur, chagrin, maussade» (FEW 10, 137a sous reburrus; VerrAnj). Extension sémantique de prendre qqn à rebrousse-poil, "maladroitement, de telle sorte qu'il se rebiffe». Etre à rebrousse-poil a été relevé dans les années 1970 (Lavoie 2879 : «être de mauvaise humeur» et 2920 : «être en colère»). V. aussi Aspects phonétiques sous brousse-poil ( $\grave{a} \sim)$.

regriche ou griche-poil (à ) (Ms. 1 Cahier B); «[à rebrousse-poil]». De regricher, d'abord attesté comme participe adjectif au XVIe s. : regrissé, «qui a le poil hérissé». Cf. en Normandie, gricher, «être de mauvaise humeur». Le sème de "mauvaise humeur" est très présent dans les mots de cette famille, dans les parlers du Nord et du Nord-Ouest (FEW 16, 393a-b sous $\left.{ }^{*} k r i s a n\right)$. Figure dans Glossaire (sous regrichepoil (à ) : «brosser à regriche-poilı). Les deux formes ont été relevées en Acadie au sens de "caresser un animal à rebrousse-poil» (Massignon 992). V. Griche-poil.

regricher (Ms. 1 Cahier B); "[se hérisser (des cheveux)]". Ce sens a été relevé dans les parlers du Maine, de l'Anjou et dans l'Orléanais (FEW 16,393 b sous ${ }^{*} k r i s a n$; VerrAnj; MartVend). Consigné dans Dionne et Glossaire. Recueilli comme adjectif lors d'enquêtes orales au Québec 
(PPQ 2115 : regriché, «ébouriffé») et en Acadie (Massignon 1455). Le mot est également connu des parlers louisianais, «se rebrousser, en parlant des cheveux, du poil» (DitchyLouis).

reintier (Ms. 1 Cahier A; Ms. 2); «reins». Dérivé dialectal de reins au sens de «lombes, bas de l'épine dorsale et la région voisine»; largement connu des parlers du Nord-Ouest et du Centre (FEW 10, 248b sous ren). Consigné dans Dunn, Clapin (sous reinquier), Dionne et Glossaire (sous reinquier et reintier). Recueilli lors d'enquêtes orales au Québec (PPQ2121s, «région lombaire»; Lavoie 2371, «colonne vertébrale», 2375, "rein») et en Acadie (Massignon 1514). Connu des parlers franco-américains de la Louisiane et du Missouri (DitchyLouis sous reintier; DorrSteGen sous reinquier).

relevée (Ms. 1 Cahier $A$; Ms. 2); «tems de l'après-dinée». Le mot est substantivé au $\mathrm{XII}^{\mathrm{e}}$ s. et désigne, par allusion, le moment où l'on se relève après la sieste (ReyHist sous relever). À partir du XVII ${ }^{\mathrm{e}}$ s., relevée se spécialise comme terme de la langue juridique; figure en français général de 1636 à Académie 1935 : «seulement terme de procédure ou de pratique, dans séance de relevée». Largement connu au sens d'«aprèsmidi» dans les parlers normands, bretons romans et bas-manceaux (FEW 5, 278a sous levare). Attesté, en Nouvelle-France, en 16561657 dans le Journal des jésuites : «a deux heures de la Relevéen (cité dans Massignon 1391). Consigné dans Clapin («En France, ce mot n'est plus usité qu'au palais de justice») et Glossaire. Recueilli lors d'enquêtes orales au Québec (Massicotte I-15; PPQ1710; Lavoie 27) et en Acadie (Massignon ib.). V. Après-dinée.

résous (Ms. 1 Cahier A; Ms. 2). V. Aspects morphologiques.

[retaper] (Ms. 1 Cahier B sous baiser et pincettes); «[tromper]». Le verbe est attesté en français général dans : il a été bien retapé au sens de «fort maltraité, critiqué, tourné en ridicule», enregistré dans Académie de 1798 à 1878 (FEW 13/1, 103a sous tapp-). Le sens de «tromper, rouler qqn" est une extension sémantique qui semble n'avoir eu cours qu'en français canadien (FEW 103b). Potier le relève au Détroit en 1748 (HalPot 294). Consigné dans Glossaire. Recueilli lors d'enquêtes orales dans les années 1970 (PPQ1791x). V. Baiser, Baiser en guedou et Baiser en pincettes.

retontir (Ms. 1 Cahier A); "rebondir (faire des bonds)». Dans ce sens, le verbe a été relevé en Touraine (FEW 13/2, 418a sous tunt-). Cf. également dans les parlers poitevins : retontiner, "rebondir, en parlant d'une balle ou d'un ballon» (MinPoit). Consigné dans Dunn, Clapin, Dionne et Glossaire. Recueilli lors d'enquêtes orales au Québec dans les années 1970 (PPQ2168; Lavoie 2417) et encore courant (DQA). 
retontir (Ms. 1 Cahier A); «retentir». Attesté au XIVe s. en wallon sous la forme retondir. Consigné sous cette forme en français général de 1515 à 1660; relevé sous les deux formes retondir et retontir en Bretagne romane, en Normandie, en Saintonge, en Poitou, en Champagne, ainsi qu'en langue d'oc (FEW 13/1, 345b sous tinnire et FEW 13/2, 418a sous tunt-). Consigné dans Dunn, Clapin, Dionne et Glossaire. Encore courant au Québec (DQA).

[retraire] (Ms. 1 Cahier A; Ms. 2 sous retraiter); «retirer par droit de parenté ou par droit Seigneurial, un héritage qui a été vendu» (v. plus loin retraiter et retrayer). Retraire a eu cours en français, du XIII ${ }^{\mathrm{e}} \mathrm{s}$. à Académie 1694 : «retirer un bien des mains d'un acquéreur (en lui en remboursant le prix)»; figure dans Académie 1718 à 1935 avec la remarque : "on dit plutôt retiren (FEW 10,343a sous retrahere).

retraiter (Ms. 1 Cahier A; Ms. 2); «retirer par droit de parenté ou par droit Seigneurial, un héritage qui a été vendu» (v. retraire et retrayer). De retrait (FEW 10, 343a sous retrahere); retraiter ne semble pas avoir été relevé ailleurs dans ce sens.

retrayer (Ms. 1 Cahier A; Ms. 2); «faire un retrait». Même sens que retraire et retraiter (v. ces mots plus haut). Retrayer est attesté au $\mathrm{XV}^{\mathrm{e}}$ s., à la forme pronominale, au sens de «se retirer» (FEW 10, 341a sous retrabere). V. également Aspects morphologiques.

rhimb de vent (Ms. 1 Cahier B); «rhumb de vent». Terme de marine. D'abord attesté sous la forme ryn de vent, «rumb», au XV $\mathrm{XV}^{\mathrm{e}}$ s.; rim de vent figure en français de 1573 à 1606 (FEW 16, 719b sous rim). Forme empruntée à l'anglais rim. L'altération, en français, de ryn en rumb (1553) s'est faite sous l'influence de l'espagnol rombo. En français général, aire de vent et quart de vent ont supplanté l'ancien rum de vent (ReyHist sous rhumb). Figure dans Dionne (sous rin de vent) et Glossaire (sous rain-de-vent), qui donnent au mot, outre l'acception maritime, le sens large de «direction». Recueilli dans les années 1970 avec l'acception de «saute de vent» (PPQ 1191; Lavoie 80).

robe de bœuf (Ms. 1 Cahier A); "peau de buffle préparée d'un seul côté». Au sens de "peau", robe est attesté en ancien provençal : rauba de buou, "peau de bœuf». En français général, robe est attesté depuis 1640 avec l'acception de "pelage (de certains animaux, cheval, bœuf)»; également connu dans robe de laine, "quantité de laine qu'on lève en tondant un mouton", syntagme qui a figuré dans les dictionnaires de Trévoux 1752 à Larousse 1875 (FEW 16, 675b sous ${ }^{*}$ rauba; ReyHist). Consigné dans Clapin (sous robe : robe de buffalo) et Glossaire (sous robe : robe de buffle). Recueilli lors d'enquêtes orales dans les années 1970 (PPQ1102B : robe de buffalo). Le syntagme a été usité en anglais 
canadien dès 1775 buffalo robe (DictCan). Robe a été plus largement usité dans robe de carriole. Figure dans Clapin, Glossaire, DQA ( de carriole, couverture de voyage en fourrure, en peau, dont on se servait l'hiver»).

Les termes bouuf, buffle et buffalo désignent le Bison bison, un bovidé de l'Amérique du Nord, connu sous le nom de bison. Les termes bauf (bouf illinois, bouf sauvage) et buffle sont usités concurremment aux $\mathrm{XVII}^{\mathrm{e}}$ et XVIII $\mathrm{e}$ s. dans les relations de la Nouvelle-France (BlaisTop 23). Buffalo est le terme anglais.

rustique (Ms. 1 Cahier A); «[tenace (en parlant d'une personne); difficile, épineux, fatigant (en parlant de qqch.)]». Il s'agit probablement d'une extension de rustique au sens dialectal de "vigoureux, solide, robuste», largement attesté dans les parlers du Nord-Ouest et du Centre (FEW 10, 593a sous rusticus). En parlant d'une personne, rustique a été relevé au sens de «rusé, intelligent» en Saintonge (MussSaint) et en Anjou avec la signification de «décidé, hardi» (VerrAnj : «Se dit d'un gaillard ou d'une femme qui a la langue bien affiléem). Dionne l'enregistre au sens de "difficile à vivre». Recueilli au Québec dans les années 1970 (PPQ 2179A : «vigoureux, résistant, en santé»).

sarpidon (Ms. 1 Cahier A; Ms. 2); «tapageur». Le mot est attesté, sous les formes serpidas, serpida, en Bretagne romane où il a le sens d'«enfant turbulent, malin, bruyant"; en Anjou, serpida a été relevé comme adjectif et signifie "effronté» (FEW 11, 522b sous serpens). Figure dans Dionne (sous sarpida : "enfant effronté") et Glossaire (sous sarpidon, sarpida et serpida). Le mot est aujourd'hui sorti d'usage. V. aussi Aspects phonétiques et Aspects morphologiques.

[sauvage] (Ms. 1 Cahier A et Ms. 2 sous micoine et soulier; Ms. 2 sous boucaner); subst., "[Amérindien]»; adj., "[relatif aux Amérindiens]». Le mot a cours en français général depuis le début du XIII' $s$. au sens de "qui vit en dehors des sociétés civilisées (de peuples, de gens)». Le substantif est attesté depuis 1596 (FEW 11, 617b sous silvaticus; ReyHist). Ce sens est vieilli de nos jours en français général (PRob 1993; PLar 1998) comme en français québécois (DQA). Sauvage est le seul mot qu'emploie Viger pour parler des Amérindiens et de leur culture. Le terme était usuel dans les écrits de la Nouvelle-France, et il l'est resté en français québécois jusqu'au $\mathrm{XIX}^{\mathrm{e}} \mathrm{s}$., époque à laquelle il entre en concurrence (probablement sous l'influence de l'anglais) avec Indien. Amérindien est aujourd'hui le terme usuel (DQA).

sauvagesse (Ms. 1 Cahier A; Ms. 2); «une sauvage, une femme sauvage». Attesté dès 1632 chez Sagard. À partir du XIX ${ }^{\mathrm{e}}$ s., le mot est relevé dans les dictionnaires avec la mention "vieux ou ironique» (FEW 11, 
618a sous silvaticus; ReyHist sous sauvage; PRob 1993). Figure dans DQA (sous sauvage : «Autrefois. Femme amérindienne»). Le mot est vieux et péjoratif de nos jours au Québec. Une sauvage a figuré sans mention dans les dictionnaires d'Académie 1762 à DG (FEW 11, $617 \mathrm{~b})$.

[savonner] (Ms. 1 Cahier B et Ms. 2 sous brâsser); «réprimander». D’abord attesté dans la locution savonner la tête à qqn, depuis 1669. Les dictionnaires donnent savonner qqn comme «populaire» ou «familier» (Bescherelle 1858, Littré, DG). Présent de nos jours dans la locution vieillie savonner la tête de qqn (TLF; ReyHist sous savon; PRob 1993).

[scier les bleds] (Ms. 1 Cahier A sous couper); «couper les bleds à l'aide de la faucille». Le verbe est largement attesté dans les parlers galloromans sous de nombreuses formes, dont seter, seer, seyer, soyer, etc. (FEW 11, 363b-364a sous secare). Trévoux 1771 le relève : «Scier se dit aussi des blés qu'on coupe avec des faucilles qui ont de petites dents comme des scies. Quelques-uns en ce sens, disent soyer ou scier; mais les honnêtes gens disent scier." Le mot est vieux ou dialectal (GLLF; TLF; ReyHist). Couper à la faucille était l'expression utilisée lors d'enquêtes effectuées dans les années 1970 (PPQ 839; Lavoie 1068).

sentinelle (Ms. 1 Cahier A). V. Aspects morphologiques.

sieau (Ms. 1 Cahier A; Ms. 2). V. Aspects phonétiques.

[soc] (Ms. 1 Cahier A et Ms. 2 sous reintier); «reintier d'un cochon». Ne semble pas avoir été relevé dans ce sens ailleurs qu'en français canadien. Potier le relève au Détroit : Le Soc d'un cochon \{le rable (HalPot 298). Recueilli dans les années 1970 (PPQ 581, «échinée»; Lavoie 3150, «échine de porc»). Usuel encore au Québec comme terme de boucherie (DQA : "Morceau de viande de porc qui provient de l'épaule»).

sorcière (Ms. 1 Cahier $\mathrm{A}$ ); «tourbillon de neige ou de poussière, occasionné subitement par un fort vent, et de peu de durée». Le mot, dans ce sens, est un apport des parlers angevin, poitevin et saintongeais (FEW 12, 121a sous sors; MinPoit; VerrAnj : «tourbillon de vent attribué aux sorciers»; RézOuest). Consigné dans Dionne et Glossaire. Recueilli lors d'enquêtes orales dans les années 1970 (Massicotte I-167; PPQ 1192; Lavoie 81); recueilli également en Acadie (Massignon 94).

Sorel (Ms. 1 Cahier A); «habitant de Sorel». Gentilé qui n'a pas été relevé par les glossairistes québécois. La forme actuelle, Sorelois, date de 1876 (DugGent; DQA). 
soulier (Ms. 1 Cahier A; Ms. 2); souliers franfois, «souliers de cuir qui nous viennent de l'étranger et que les cordonniers manufacturent». FEW relève cet emploi canadien : «chaussures visant à l'élégance» (FEW 3, 751a sous France). Consigné dans Clapin (sous français (souliers $\sim$ ): «Dans les premiers temps de la colonie, on appelait ainsi le soulier à boucles, importé de France»), dans Glossaire : «souliers avec semelles fabriqués par les cordonniers (par opposition à souliers de beuf, à souliers mous, à souliers sauvages, etc.)m. Recueilli lors d'enquêtes orales au Québec (PPQ1776x) et en Acadie (Massignon 1655).

Souliers de bauf, "ceux que nos habitans se font de la peau du bœuf". FEW consigne cet emploi canadien : «bottes sans semelles» (FEW 12,364 a sous *subtelare). Consigné dans Clapin : «Bottes sans semelles, ou gaillards, confectionnées avec du gros cuir auquel on a laissé sa couleur naturelle». Recueilli lors d'enquêtes orales dans les années 1970 (PPQ 2078 : «mocassins pour aller en raquettes»; Lavoie 2671 : «chaussure sans semelle fabriquée avec du cuir de bœuf»). Recueilli en Acadie (Massignon 1669).

Souliers sauvages, "ceux que nos Sauvages font de la peau des bêtes fauves qu'ils tuent, telles que le chevreuil, le caribou, l'orignal». Attesté en Nouvelle-France dès 1643 (cité dans Massignon 1669). Figure dans Glossaire : "chaussures molles, ordinairement sans semelles». Recueilli lors d'enquêtes orales au Québec (PPQ 2078 : «mocassins pour aller en raquettes»; Lavoie 2670 : «chaussure sans semelle faite de peau d'orignal, de chevreuil ou de caribou»), et en Acadie (Massignon ib.).

soupe (dormir comme une $\sim$ ) (Ms. 1 Cahier A); «[dormir profondément]». L'expression a été relevée en français général en 1739; variante de dormir comme une souche. Les nombreuses expressions qui contiennent le mot soupe expliquent sans doute cette variante (NisPar 93; DunBouq).

souris-chaude (Ms. 1 Cahier A); «chauve-souris». Les formes attestées sont chauve-souris, souris-chauve; chaude-souris et souris-chaude. Attesté dès le XIII $\mathrm{e}$ s., caude soris. Souris-chaude est très largement connu des parlers d'oil et d'oc (FEW 12, 112a-b sous sorix). Recueilli lors d'enquêtes orales dans les années 1970, où souris-chaude est plus fréquent que chauve-souris (PPQ 1597; Lavoie 673); recueilli également en Acadie (Massignon 390).

stellà (Ms. 1 Cahier A). V. Aspects morphologiques.

stellci(t) (Ms. 1 Cahier A). V. Aspects morphologiques. 
sucet (Ms. 1 Cahier A); «tuyau du blé d'Inde, ou sa tige, dégarnie de ses épis et que l'on fait manger au bétail à cornes». Il s'agit probablement d'un mot des parlers de l'Ouest. Cf. en Vendée, soucès : «restes» (FEW 12,379 a sous succedere) et souchin : «foin de bonne qualité» (RézVend). Dans les parlers du Nord-Ouest, sucet désigne le "chèvrefeuille» (FEW 12, 389b sous suctiare). Le mot est attesté en Nouvelle-France avant 1725 (MorHist 198 : suces de bled'Inde). Consigné dans Glossaire. Recueilli lors d'enquêtes orales dans les années 1970, mais moins fréquent que son synonyme coton (PPQ 892). V. Coton.

sur (Ms. 1 Cahier A; Ms. 2). V. Aspects syntaxiques.

suspect, ecte (Ms. 1 Cahier A; Ms. 2); «susceptible». Dans ce sens, suspect semble n'avoir été relevé qu'en français d'Amérique (FEW 12, 470b sous suspectus). Consigné dans Clapin (sous suspec) et Glossaire. Recueilli lors d'enquêtes orales dans les années 1970 (PPQ 1837A, 2310) et encore vivant de nos jours. Attesté dans les parlers francoaméricains du Missouri (DorrSteGen sous suspec) et de la Louisiane (DitchyLouis; ReadLouis).

tapin (Ms. 1 Cahier A; Ms. 2); «tape légère, soufflet». Relevé au sens de «gifle» en 1790; connu des parlers picards, normands, bourguignons, wallons et suisses romands (FEW 13/1, 100b sous tapp-). Figure dans Dionne, qui a relevé le mot dans les Mémoires de Philippe Aubert de Gaspé. Recueilli en franco-ontarien (LemVieux 9, 151).

tapon (Ms. 1 Cahier A; Ms. 2); «tas, paquet, touffe». Tapon est attesté, depuis Furetière 1690, au sens d' 'étoffe ou autre matière chiffonnée et formant une sorte de boule compacte»; sens donné, de nos jours, avec la mention «vieilli» ou «familier» (PRob 1993; PLar 1998). Le sens large attribué à ce mot, en français québécois, vient notamment des parlers du Nord-Ouest, du Centre et de la Bourgogne, où le mot est attesté avec les acceptions de «morceau (de qqch. de mou, viande, etc.)", "petite masse en désordre», "petit amas", «motte de beurre, de laine", "poignée, tas» (FEW 17, 308b-309a sous "tappo).Tapon est encore vivant en Touraine où il signifie, entre autres, "petit amas de fibres, d'herbe, de farine, etc.» (SimTour). Consigné dans Dionne et Glossaire. Recueilli lors d'enquêtes orales dans les années 1970 (PPQ et Lavoie, qui donnent de très nombreux emplois du mot). Toujours en usage au Québec (DQA : tapon de poussière, tapon de journaux, tapon de nuages noirs, de neige, de terre, tapons, "grumeaux»). Recueilli également en Acadie (Massignon 1326 : "grumeaux»).

Tapon, «petit enfant gros et gras», est connu en Sologne («gros enfant») et en Anjou («individu ou animal petit et râblé») (FEW 17, 309 a sous ${ }^{*}$ tappo). Glossaire l'enregistre au sens de "grosse femme». 
tauraille (Ms. 1 Cahier A); «jeune veau, génisse (taure)». Dérivé de taure. Bien attesté au sens de "génisse» dans les parlers normands, beaucerons, angevins, orléanais et saintongeais (FEW 13/1, 132b sous taurus). Consigné dans Dionne («petite taure»), Glossaire (sous taurâille: «jeune bœuf, génisse»). Relevé lors d'enquêtes orales dans les années 1970 (PPQ 506A, «veau femelle», et 507, «génisse»). Recueilli en Acadie (Massignon 837, «génisse»).

[taure] (Ms. 1 Cahier A sous tauraille); «génisse». Attesté dès le XVI ${ }^{\mathrm{e}}$. Terme d'élevage. Très largement attesté en France d'oil et d'oc (FEW $13 / 1,132 \mathrm{~b}$ sous taurus). Donné aujourd'hui comme terme régional ou technique (PRob 1993; PLar 1998). Recueilli dans les années 1970, surtout pour désigner la génisse de un à deux ans (PPQ507; Lavoie 1312).

tête de boule (Ms. 1 Cahier A); "personne entêtée, opiniâtre». Fait peutêtre allusion à la tribu amérindienne de ce nom. Selon Kalm, ce nom leur a été attribué en raison de leur stupidité (Kalm 838). Bougainville en 1758 les décrit ainsi : «Ils ont peu d'idées et peu de mots, nulle prévoyance de l'avenir" (RAPQ1923-1924, 325).

thétière (Ms. 1 Cahier A). V. Aspects phonétiques.

tinton (Ms. 1 Cahier B; Ms. 2); «son lent d'une cloche dont le battant ne touche que d'un côté». Attesté au $X^{\mathrm{e}}$ s., tinton, «bruit», et dans Cotgrave 1611 au sens de «son d'une cloche» (FEW 13/1, 347a sous tinnitare). Potier l'enregistre au Détroit en 1744 (HalPot 301). Recueilli lors d'enquêtes orales dans les années 1970 (PPQ 1896, "glas"; 2310, «dernier coup de cloche avant la messe»).

tirans (Ms. 1 Cahier A); «aurore boréale». Extension sémantique de tirants, "rayons qui annoncent du mauvais temps", attesté en Normandie, «rayons solaires filtrant à travers les nuages» en hautmanceau et "raies formées par la pluie qu'on voit tomber au loin" en Moselle (FEW 6/1, 399b sous martyrium). Consigné dans Dionne et Glossaire (sous tirant). Recueilli dans les années 1970 (PPQ 1161; Lavoie 66). V. Clairon.

tire (Ms. 1 Cahier A); «sorte de sucre en bâton que l'on procure de la mélasse, en la faisant bouillir». Déverbal de tirer (le produit à demi durci est étiré). Ce sens est une innovation québécoise (ReyHist sous tirer). Consigné dans Dunn : «Mélasse ou sirop à demi durci sur le feu, et ensuite bien étiré avec les mains". Recueilli lors d'enquêtes orales dans les années 1970 (PPQ 242B : tire (de la) Sainte-Catherine, tire à la mélasse). Encore courant (DQA : "Confiserie de consistance molle faite avec de la mélasse ou du sirop de cassonade qu'on fait cuire. $L a$ tire (de la) Sainte-Catherine»). 
Bâton de tire, «[tire étirée en bâtonnet]». Recueilli en 1970 (PPQ 242B : tire en bâton).

Tire est également connu dans tire d'érable : «confiserie de consistance semi-liquide, de couleur brune et translucide, provenant de la concentration du sirop d'érable” (DQA; Massicotte V-220).

tondre (Ms. 1 Cahier A); subst. masc., «[amadou]». Le mot est attesté, au féminin, au sens d' "amadou» dès le XII' ${ }^{\mathrm{e}}$ s. en ancien normand. Relevé en Picardie, en Normandie, en Bretagne romane, en Aunis et en Saintonge (FEW 17, 387a sous tundr). Figure dans Littré au féminin. Tondre est attesté en Nouvelle-France dès 1634 : "un morceau de tondre allumés (cité dans Massignon 324). Potier l'enregistre à Lorette en 1743-1744 (sans genre) (HalPot 22). Recueilli au masculin dans les années 1970 (PPQ 91x; Lavoie 2013). Connu également en Acadie (Massignon ib.), en franco-ontarien (LemVieux 19,164) et en Louisiane (ReadLouis). V. Aspects morphologiques.

touche (fumer une ) (Ms. 1 Cahier A; Ms. 2); "fumer un peu et tout au plus une pipe pleine». Attesté dans le parler boulonnais au sens d'«aspiration qu'un fumeur tire de sa pipe à chaque coup de langue» : funmer eune touche, funmer quiques touches (HaignBoul). Cf. également en picard, touke, "prise de tabac", et en béarnais, toucha, "priser du tabac" (FEW 13/2, 7a sous tokk-). Consigné dans Dunn, Clapin et Dionne (Tirer une touche), Glossaire (fumer, tirer une touche). Recueilli dans les années 1970 : fumer, prendre, tirer une touche (PPQ2070; Lavoie 3131). Encore en usage (DQA : «bouffée de cigarette ou de pipe»). L'expression devait être assez répandue puisqu'elle est attestée, en outre, dans les parlers réunionnais et martiniquais (ChaudRéun II, 881).

tourniquet (Ms. 1 Cahier B); «tournoiement d'eau dans un trou fait dans la glace d'une rivière, particulièrement le printems". Ne semble pas avoir été relevé ailleurs qu'en français canadien; cf. les sens de «filet mis en mouvement par l'action de l'eau" et "gyrin, coléoptère qui tourne sur l'eau" (FEW 13/2, 58a sous tornare). Consigné dans Clapin et Dionne (sous tourniquet), Glossaire (sous tourniquette). Recueilli lors d'enquêtes orales dans les années 1970 (Lavoie 209).

train (Ms. 1 Cahier A; Ms. 2); «occupations quotidiennes du ménage». Train est attesté au sens de "genre de vie, manière d'agir» au XII ${ }^{\mathrm{e}} \mathrm{s}$. Relevé en Bretagne romane : faire son train, ufaire son ménage" (cité dans Massignon 1218); en Savoie : faire son train, «s'occuper des travaux habituels du ménage»; en Alsace : "train d'une maison»; et en Suisse romande : «travaux qu'exige un domaine» (FEW 13/2,163b et $164 \mathrm{~b}$ sous ${ }^{*}$ traginare). Consigné dans Glossaire. Recueilli dans les années 1970, souvent dans le syntagme train de la maison (PPQ273 : 
«ménage de chaque jour»; Lavoie 2047 : «ménage»). Recueilli en Acadie (Massignon 1218 : «faire du ménage»).

train (en ) (Ms. 1 Cahier A; Ms. 2); «ivre». Extension de mettre en train, "exciter au plaisir, mettre en bonne disposition", attesté depuis Cotgrave 1611, et d'être en train, «être en bonne disposition, de bonne humeur", expression enregistrée dès le début du XVII s. En train, «légèrement ivre», a été relevé dans les parlers normands, poitevins et lorrains; attesté en français général de 1872 à 1933 (FEW 13/2, 164b sous ${ }^{*}$ traginare). Consigné dans Dunn, Clapin et Dionne. Recueilli dans les années 1970 (PPQ267 : "un peu ivre»). V. Datations.

traîne (Ms. 1 Cahier A); «sorte de voiture d'hyver, qui sert aux habitans pour charrier le bois, les denrées, le foin, etc.; [et pour] Voyager». Attesté dès le XII ${ }^{\mathrm{e}}$ s. en langue d'oc (Quercy), au sens de «traîneau pour le transport de lourds fardeaux». Le mot a eu cours en français général de 1457 à 1636 . Il a été relevé entre autres dans les parlers du Nord-Ouest, de l'Ouest et du Centre (FEW 13/2, 165a sous *traginare). Potier le relève à Lorette en 1743-1744 (HalPot 302). Recueilli lors d'enquêtes orales en 1970 (Massicotte V-196; PPQ 1089B) et encore usité (DQA sous traîner : «Voiture sur patins bas qui glisse sur la neige ou la glace, qui sert au transport (du bois, des marchandises...)m). Cette traine est souvent munie de bâtons fixés à la verticale de chaque côté de la plate-forme d'où son nom de traîne à bâtons (CorrVoit 151 et LeclVoit 9-11). Recueilli en Acadie (Massignon 668).

traîne de clisse (Ms. 1 Cahier A); "voiture d'hyver des Sauvages, qui leur sert à transporter leur venaison et autres choses. C'est une planche très mince, courbée par devant». Recueilli dans les années 1970 (PPQ 1103 : traine de clisse, traine d'éclisse). Ce type de traineau long et étroit, sans patins est nommé, de nos jours, traine sauvage, tabagane ou tobagane (PPQ1103; DQA sous traîner).

Clisse variante d'éclisse. Le mot est attesté au $\mathrm{XI}^{\mathrm{e}}$ s. sous la forme esclice et sous celle de clice au XII ${ }^{\mathrm{e}}$ s., au sens d'«éclat de bois allongé». Au $\mathrm{XIV}^{\mathrm{e}}$ s., en Anjou, sous la forme cliche et sous celle de clisse au XVI ${ }^{\mathrm{e}}$ s., le mot désigne du «bois de fente servant à des ouvrages légers». Connu des parlers picards et normands sous la variante cliche (FEW 17, 152a sous * slitan). Les deux formes (clisse et éclisse) sont connues au Québec (DulDictC sous clisse et éclisse).

traîneau (Ms. 1 Cahier A); «traine des Canadiens, mais d'une dimension beaucoup plus petite, assez souvent avec des bras. Elle sert aux enfans à courir et glisser sur la neige». Spécialisation de traineau, «voiture à patin que l'on traîne (ou pousse) sur la neige» (ReyHist sous traîner). 
Dunn l'enregistre avec ce commentaire : «Le plus petit de nos véhicules d'hiver, auquel, à la campagne, on attelle les chiens, et qui sert aux enfants pour tirer des glissades»; Bélisle ${ }^{3}:$ :Petite voiture appelée luge en France». Recueilli dans les années 1970 (PPQ1103) et encore en usage (DQA sous traîner).

travail (Ms. 1 Cahier A); «brancard». Extension du sens technique de "machine à trois ou quatre piliers entre lesquels les maréchaux attachent les chevaux vicieux pour les ferrer ou pour les panser», attesté dès le XIII ${ }^{e}$ s. Le sens de "brancard" ne semble pas avoir été relevé ailleurs qu'en français canadien (FEW 13/2, 291b sous tripalium; ReyHist sous travailler). Connu en Nouvelle-France dès 1724 (JunLex 99). Recueilli lors d'enquêtes orales dans les années 1970, où il est bien attesté, mais un peu moins fréquent que menoirs (PPQ 1119). V. Menoirs.

trèfe (Ms. 1 Cahier A). V. Aspects phonétiques.

trempe (Ms. 1 Cahier A; Ms. 2); «trempé». Largement attesté dans les parlers de l'Ouest, du Centre, en Bourgogne et en franco-provençal. Féraud 1788 l'enregistre avec la mention «barbarisme provençal» (FEW 13/1, 169b sous temperare). Consigné dans Clapin, Dionne et Glossaire. Encore en usage (DQA sous tremper). Recueilli en Acadie (Massignon 8 «(terrain) détrempé»).

Tout trempe, «extrêmement mouillé», relevé dans Clapin et Glossaire. Encore vivant (DQA).

Trempe comme une soupe, «très mouillé». L'expression est attestée en français général depuis Académie 1798 : trempé comme une soupe (FEW 17, 285a sous suppe; ReyExpr sous soupe).

tricoler (Ms. 1 Cahier A; Ms. 2); «[chanceler (d'un homme ivre)]». Attesté en Bretagne romane et dans les parlers de l'Ouest (FEW 17, $258 \mathrm{~b}$ sous ${ }^{*}$ strikan). Consigné dans Dunn, Clapin, Dionne et Glossaire. Recueilli lors d'enquêtes orales dans les années 1970 (PPQ $268 \mathrm{x} ; 2153 \mathrm{x})$.

troisse (Ms. 1 Cahier A). V. Aspects phonétiques.

trompe (Ms. 1 Cahier A); "petit instrument de fer, qui a une languette au milieu dont on tire un son en le mettant entre les dents et en le touchant avec le bout du doigt». "Trompe est passé en français pour désigner un instrument de musique à vent : d'abord une trompette, puis un petit instrument à bouche qui vibre (guimbarde)» (ReyHist). Consigné dans les dictionnaires, de Richelet 1680 à Académie 1878 (FEW 17, 376a sous trumba). Le mot était déjà moins usuel dans la seconde moitié du $\mathrm{XIX}^{\mathrm{e}}$ s.; Bescherelle 1858 précise : «On dit plus 
ordinairement guimbarde.) Recueilli encore dans les années 1970, mais beaucoup moins fréquent que bombarde (PPQ 2060). Connu en Acadie (PoirAcad). V. Bombarde.

tuer (Ms. 1 Cahier A; Ms. 2); «éteindre». Tuer, «éteindre (le feu, une chandelle)", est attesté depuis le XVe s. Figure dans Académie 1694 à 1762 : «Se dit quelquefois pour Esteindre.» Bescherelle 1858 le donne comme "provincial», tandis que Littré et DG l'enregistrent sans mention. Bien attesté dans les parlers du Nord-Ouest, de l'Ouest et du Centre (FEW 13/2, 446a sous tutari). Consigné dans Dunn, Clapin et Dionne. Recueilli lors d'enquêtes orales au Québec (PPQ 96; Lavoie 2037) et en Acadie (Massignon 1216). Aujourd'hui sorti d'usage.

tuque (Ms. 1 Cahier A; Ms. 2); «vêtement de tête de nos habitans; c'est un bonnet de laine tricottée». Emploi métaphorique de tuc, tuque en usage dans les parlers du domaine d'oc, notamment en gascon où il a le sens de «montagne, hauteur» (FEW 13/2, 398b sous *tukka). Le mot tuque a été également employé comme terme de marine pour désigner une "espèce de tente ou d'abri qu'on élève à l'arrière d'un vaisseau", probablement un emploi métaphorique du sens de "montagne» (FEW 23, 102a-b). Tuque est attesté en Nouvelle-France dès 1726 (JunLex 224-226). Toujours en usage en français québécois (DQA). V. Mâle.

verbalement (Ms. 1 Cahier A; Ms. 2); «[avec vivacité, rudesse]». Dans cette acception, le mot est probablement à rapprocher du sens saintongeais de "tout droit, directement" (MussSaint).

veuilloche (Ms. 1 Cahier A); «petite meule de foin». Le mot est attesté au $\mathrm{XV}^{\mathrm{e}}$ s. en ancien Poitou : velloche, «meule de foin». Veuilloche (connu aussi sous la variante veillotte) est largement relevé dans les parlers du Nord-Ouest, de l'Ouest et du Centre (FEW 14, 555a-b sous viticula). Consigné dans Dunn (sous veillotte), Clapin (sous veilloche, veillotte), Dionne (sous veilloche) et Glossaire (sous vailloche, veilloche). Recueilli lors d'enquêtes orales au Québec (Massicotte III-144; PPQ 814A, 814B; Lavoie 1137) et en Acadie (Massignon 703). V. Mulon.

vire-l'œil (Ms. 1 Cahier A; Ms. 2); subst., «[personne qui] louche d'un œil». Relevé comme adj. au sens de "qui louche», dans le Bourbonnais (FEW 14, 394a sous vibrare). Mot disparu de l'usage au Québec. V. Bicleux et Loucheur.

[voie] (Ms. 1 Cahier A; Ms. 2 sous voyage); "[quantité transportée]». Ce sens est attesté dès le début du XVI ${ }^{\mathrm{s}}$ s, " "mesure équivalant à une charretée» (FEW 14, 378a sous via). Voie qui s'employait dans voie d'eau, voie de charbon, voie de bois a disparu avant la fin du $\mathrm{XIX}^{\mathrm{e}} \mathrm{s}$. (ReyHist). 
voyage (Ms. 1 Cahier A; Ms. 2); «voie». «Il s'est employé par métonymie pour désigner ce que l'on transporte en un voyage, c'est-à-dire une charretée, d'où le sens (1872) de «charge transportée» (cf. voie) qui a disparu» (ReyHist). Bien attesté en franco-provençal (FEW 14, $382 \mathrm{~b}$ sous viaticum). Consigné dans Dunn, Clapin et Dionne. Encore bien vivant (DQA). Recueilli en Acadie (Massignon 711), en Louisiane (ReadLouis; DitchyLouis) et à la Réunion : un voyage d'eau (ChaudRéun II, 675).

[voyageur] (Ms. 1 Cahier A sous jument); hist., «[personne mandatée pour conduire des expéditions à des postes de traite désignés, dans le but de faire la traite des fourrures avec les Amérindiens]». Le mot prend cette acception à la fin du XVII ${ }^{e}$ s. avec l'expansion et l'organisation du commerce des fourrures. Le terme est entré en anglais canadien en 1793 , où il est également orthographié voyager (DictCan). Connu également en anglais américain (Mathews). Relevé en franco-américain du Mississippi (McDermMiss).

vulgaire (Ms. 1 Cahier A; Ms. 2); «visible, évident». Attesté en moyen français : être vulgaire, adj., «trop bien connu de tout le monde»; relevé dans le langage de Paris au sens de «visible" et dans les parlers normands, hauts-manceaux et alsaciens au sens d'eévident" (FEW 14, 642b sous vulgaris). Consigné dans Glossaire.

\section{EMPRUNTS AUX LANGUES AMÉRINDIENNES}

achigan (Ms. 1 Cahier B); "[gros poisson d'eau douce de la famille des Microptéridés]». Le mot est issu des langues de la famille linguistique algonquienne. Cuoq le définit ainsi : «espèce de grosse tanche dont le nom algonquin fut adopté par les colons venus de France» (CuoqAlg 12 a sous acigan). Attesté dès 1655 sous la forme acbigen (RJ XLII, 70), ouchigan chez Boucher 1664 et achigan depuis 1683. Le mot désigne une variété de perche et s'applique au Micropterus dolomieui (achigan à petite bouche) et au Micropterus salmoides (achigan à grande bouche) (MélPoiss 316). Figure dans TLF, ReyHist, PRob 1993 et PLar 1998. Attesté en anglais canadien dès 1800 (DictCan). Terme usuel pour désigner ce poisson au Québec (DQA).

apichimon (Ms. 1 Cahier A; Ms. 2); «bourrelet de linge ou de paille, que l'on met sur le cou du bouf qui labourre; morceau d'étoffe, peau de mouton, ou autre chose semblable, que nos habitans mettent en guise de Selle lorsqu'ils montent à cheval; méchant lit, grabat». Le mot est connu des langues de la famille algonquienne. Relevé dans Fabvre, au 
XVII s., au sens de «litière, lit, matelas" (Fabvre 34 sous aspichim8n). Cuoq, qui lui donne le sens de «lit, litière, literie», ajoute : "Ce mot se dit de tout ce qu'on met sous soi pour n'être pas couché sur la dure. Ainsi une paillasse, une coite, un matelas, une couverture, une peau, du foin, de la paille, des branches de cèdre, de pruche ou de sapin etc., dès qu'on les met sous soi pour être moins durement couché, sont un apicimon "(CuoqAlg 55b sous apicimon). Potier le relève avec un sème voisin : «apichimon \{ morceau d'ecorce qu'on met dans Les pinces du Canot pour Servir de marche-pied aux Canoteurs" (HalPot 219). Le mot a eu une certaine diffusion au XVIII ${ }^{\mathrm{s}}$.; il figure, en effet, sous la plume de Bougainville en 1757 : «Apichimon : terme sauvage usité dans la langue française parmi les Canadiens pour exprimer l'équipement d'hiver» (cité dans Massignon 1622). Glossaire reprend les trois sens donnés par Viger et en donne d'autres à valeur péjorative où le mot s'applique à toutes sortes de choses. Viger a écarté le sens de «chapeau de paille» qu'il lui avait d'abord donné. Recueilli en Acadie au sens de «mal mis» en parlant d'une personne (Massignon ib. : «il a l'air d'un apichimon»). Relevé en anglais canadien et américain (DictCan sous appichimon; Mathews sous apishamore). Mot aujourd'hui sorti de l'usage en français québécois comme en anglais canadien et américain.

caribou (Ms. 1 Cahier A); «renne». Désigne le Rangifer tarandus. Le mot est emprunté à une langue de la famille linguistique algonquienne, probablement au micmac Xalibu (FEW 20,60b sous kalibu). Il signifierait «pelleteur», allusion à l'habitude qu'a l'animal de creuser profondément la neige avec ses sabots (RoussBouch 307). Caribou est attesté dans les relations de la Nouvelle-France dès 1609 chez Lescarbot. Consigné dans les dictionnaires français depuis Trévoux 1721. Nom usuel de cet animal au Québec comme en Acadie (DQA; Massignon 366). Le mot est entré en anglais canadien en 1665 où il est connu sous les formes caribou et cariboo (DictCan). Le mot figure encore de nos jours sous la forme caribou en anglais canadien (Gage) et américain (Webster).

maskinongé (Ms. 1 Cahier B); «espèce de brochet». Désigne le Esox maskinongy de la famille des Ésocidés. D'origine algonquienne, maskinongé (orthographié également masquinongé) viendrait de mac, "gros", et kinonje, "brochet" (CuoqAlg sous mackinonje et maskinonje). Le mot est donné en 1680 sous la forme masquinongé (BlaisTop : sur une carte de Vachon de Belmont de 1680). Potier le relève sous cette dernière forme à Lorette en 1743-1744 (HalPot 223). Maskinongé figure dans TLF (qui donne 1709 comme première attestation) ainsi que dans PRob 1993 et PLar 1998 notamment. Le mot est entré en anglais canadien sous la forme maskalonge en 1794 (DictCan sous muskellunge). Il est usuel encore de nos jours sous les formes maskinonge et 
muskellunge en anglais canadien (Gage) et muskellunge en anglais américain (Webster). Terme usuel pour désigner ce poisson en français québécois (DQA).

micoine ou micouène (Ms. 1 Cahier A; Ms. 2); "petit vase de bois qui sert de cuiller à nos Sauvages». Le mot appartient à la famille linguistique algonquienne et a été relevé notamment en ojibwé, en cri, en naskapi, en montagnais et en micmac (Fabvre 62 sous emik8an; Lacombe 337 sous emikkwân ; Baraga 241b sous êmikwân; Piggott sous e:mikkwa:n; CuoqAlg sous emikwan). Attesté, sous la forme micouanne, dès 1695 (Massignon 1288); largement usité au XVIII ${ }^{\mathrm{e}} \mathrm{s}$. (JunPron 62). Figure dans Dunn (sous micouenne), Clapin (sous micouan, micouenne; v. également app. 361), Dionne (sous micouanne, micouenne) et Glossairc (sous micouenne). Recueilli lors d'enquêtes orales dans les années 1970 au sens de «louche», «grande cuiller de bois pour faire le beurre ou verser le sucre d'érable dans les moulesm; attesté surtout dans la région de Montréal (PPQ 168, 990). Consigné dans DQA (sous micouenne ou micoine : «Autrefois. Grande cuiller de bois ou d'écorce employée à divers usages domestiques, spécialt à la cabane à sucre»). Le mot a été relevé en Acadie (Massignon ib.) et en Louisiane (DitchyLouis).

mitasse (Ms. 1 Cahier A; Ms. 2); "sorte de vêtement en usage chez nos Sauvages, qui sert à couvrir la jambe» (définition donnée sous hausse). Mitasse est emprunté aux langues de la famille algonquienne; attesté notamment en montagnais (Fabvre 162, mitas, «bas de chausses»), en ojibwé (Piggott, mita:ss) et en cri (Lacombe, mitâs). Cuoq le fait venir du mot tas qu'il définit ainsi : "chausses des Aborigènes du Canada, ce que les colons français ont appelé mitasses»; selon cet auteur, le mot tas, qui désignait dans la langue algonquine les chausses des Amérindiens, a désigné également, par extension, les guêtres et les bas des Français : «Tant qu'il n'est pas nécessaire de distinguer avec précision de quelle espèce de chausses on parle, ce petit mot suffit pour exprimer indifféremment toute espèce de chausses [...]. Mais lorsqu'il est nécessaire de dire avec précision de quelle espèce de chausses on parle, il faut mettre devant tas, le mot qui exprime cette espèce» (CuoqAlg 383a sous mitas et tas). Relevé en Nouvelle-France dès 1669 (JunGl 158 n. 32, qui souligne que FEW 6/2,178a, l'a classé par erreur sous mit-). Cependant, comme le fait remarquer $M$. Juneau, l'influence de mitaine n'est peut-être pas étrangère à l'entrée de ce mot en français canadien (JunInv 218). Figure dans Dunn, Clapin (aussi app. 361) et Glossaire. Considéré aujourd'hui comme un terme appartenant à la culture amérindienne dans cette acception; le mot est cependant encore vivant en français québécois au sens de «chaussure de laine, de feutre ou d'étoffe portée par grand froid" et "grosse mitaine pour se protéger les mains du froid» (DQA). Il est entré en anglais canadien 
en 1732 (DictCan sous mitashes or mitasses) et en anglais américain (Mathews). Recueilli en Acadie (Massignon 1670) et en Louisiane (ReadLouis). V. Hausse.

otocas (Ms. 1 Cahier A); «[plante des marais, à baies rouges et acides et, par ext., la baie elle-même]». Le mot est probablement entré en français canadien par la langue huronne. Attesté chez Sagard dès 1632 sous la forme toca; la forme atoka est attestée depuis 1656 et otoka depuis 1757 (JunLex 91-96). Désigne le Vaccinium macrocarpon (airelle à gros fruits) et le Vaccinium oxycoccos (airelle canneberge) (MVictFI 440). Potier relève le mot à Lorette en 1743-1744 : atoca, atoka (HalPot 220). Figure sous la forme atoca dans TLF, PRob 1993 et PLar 1998. Attesté en anglais canadien depuis 1760 (DictCan). Recueilli lors d'enquêtes orales dans les années 1970; les formes otoca et atoca ont été relevées sporadiquement, la forme la plus fréquente étant ataca (PPQ1655; Lavoie 309-310). Le mot est usuel encore de nos jours (DQA sous atoca ou ataca). Dans le commerce, ce fruit est généralement nommé canneberge. V. aussi Aspects phonétiques.

\section{EMPRUNTS À L'ANGLAIS}

appointement (Ms. 1 Cahier A; Ms. 2); «emploi, charges, place». De l'anglais appointment, «an office or position» (OED; Webster; Kossler sous appoint (to) : «assigner; nommer (à un poste), d'où appointment, nomination (à un emploi, à une charge) $[. .$.$] et concr. l'emploi, la$ charge mêmes»).

appointer (Ms. 1 Cahier A; Ms. 2); "nommer à une charge ou place». Emprunt à l'anglais to appoint "name to an office or position» (OED; Webster; Gage; Kœssler sous appoint (to) : v. ci-dessus). Dunn, Clapin, Dionne et Glossaire enregistrent le terme; Dagenais (sous appointements : appointer ne veut pas dire «nommer", mais «verser des appointementsm).

belt (Ms. 1 Cahier B); «baudrier». Forme abrégée des mots anglais crossbelt et shoulder belt, «a belt worn over both shoulders, and crossing in front of the breast; also, in later use, a single belt passing obliquely across the breast» (OED).

bourgogner (Ms. 1 Cahier $\mathrm{A}$ ); «battre d'une manière honteuse, battre à plates coutures». Extension sémantique du verbe anglo-américain to burgoyne, «to capture, take prisoner». Dérivé verbal du patronyme Burgoyne, nom du général britannique John Burgoyne qui combattit les Américains et qui fut fait prisonnier à Saratoga en 1777 (Mathews 
sous burgoyne (to )). Consigné dans Glossaire. Le verbe a disparu du français québécois comme de l'anglais américain.

cheniquer (Ms. 1 Cahier A); «craindre de se faire battre». Probablement de l'anglais to sneak, "to go stealthily or furtively : slink» (Webster). Cette forme viendrait de shneak, ancienne prononciation d'origine irlandaise (Glossaire). Les parlers du Nord de la France connaissent le verbe cheniquer au sens de «boire de l'eau-de-vie», mais le lien avec le verbe québécois est difficile à établir en raison de l'écart sémantique entre les deux verbes (FEW 17, 47b sous schnick). Pour une étude plus détaillée sur l'origine et la prononciation du mot, voir BPFC vol. I, $18 ; 121-123 ; 144-146$. Disparu de nos jours.

cheniqueur, euse (Ms. 1 Cahier A); «qui chenique». De cheniquer (v. ce mot). Figure dans Clapin, Dionne et Glossaire (sous cheniquetux). Disparu de nos jours. V. aussi Aspects morphologiques.

comfortable (Ms. 1 Cahier B; Ms. 2); en français moderne, l'adjectif confortable, est un emprunt à l'anglais (depuis 1786); ce dernier l'avait lui-même emprunté au français à l'époque où ce mot signifiait "consolant, secourable», sens qui est attesté en ancien français (FEW 2/2, $1044 \mathrm{~b}$ sous confortare; ReyHist sous conforter).

Le sens de "consolant [d'une nouvelle]" a eu cours en anglais : «strengthening or supporting (morally or spiritually); encouraging, inspiriting, reassuring, cheering : your last letter was not comfortable» (OED). Dans ce sens, confortable peut également être une survivance de l'ancien sens français de "qui conforte», attesté au XII ${ }^{e}$ s. Cf. en ancien français : «Les tres gentilx et tres confortables lettres» (Godefroy). Donné dans AcCompl 1842 avec la mention "vieux» (cité dansTLF sous conforter) et dans Bescherelle 1858 : «Se disait autrefois pour Consolant.» Ces emplois n'ont plus cours au Québec.

Au sens d'«agréable [du temps, d'une journée]», confortable est également à rattacher à l'anglais : «affording or fitted to give tranquil enjoyment and content» (OED). Encore courant en français québécois.

Les sens de «qui fait plaisir, agréable à boire [d'une boisson], qui fortifie [d'une nourriture]", sont des emprunts à l'anglais : «strengthening or refreshing to the bodily faculties or organs; sustaining» (OED). Cf. Bescherelle 1858, qui considère le mot comme un anglicisme «très intelligible et très nécessaire en français" et qui donne comme exemple : nourriture confortable. V. Confortatif.

L'expression mener une vie confortable est usitée, de nos jours, en français général; confortable y a, entre autres sens, celui de «qui assure un 
bien-être, une tranquillité psychologique». Le mot n'est cependant utilisé, en français, qu'avec des noms de choses (ReyHist sous conforter; PRob 1993; PLar 1998).

coppre (Ms. 1 Cahier B); «sou (monnoie de compte, la 20 partie de la livre, valant 12 deniers)». De l'anglais copper «a copper coin; a penny or halfpenny" (OED). "Hist. in colonial times, a copper coin valued at one half-penny in York currency» (DictCan). Consigné sous la forme coppe dans Dunn : «sou (ancien cours)»; Clapin : "Mot aujourd'hui presque tombé en désuétude, et qui s'entendait autrefois couramment pour la pièce de un soun; Glossaire (sous cope).

crible (Ms. 1 Cahier B); «trains de bois flottés». De l'anglais canadien crib, "a raft of logs lashed together for floating downstream» (Gage, qui le donne comme un mot de l'anglais canadien). «Hist. in the Ottawa Valley especially, one of the small units or assemblages of logs that, in groups of 25 or 30, formed a raft of timber, used in driving from the camps to the mills or shipping points" (DictCan). Figure dans Dunn, Clapin («La plus petite partie d'un train-de-bois, ou cage [...]»), Dionne et Glossaire (sous cribe, crible). Recueilli lors d'enquêtes orales dans les années 1970 (PPQ1321B; Lavoie 561). Consigné en anglais américain (Mathews).

décent (Ms. 1 Cahier A; Ms. 2); «honorables [en parlant de funérailles]». De l'anglais decent, "proper and right $:$ a decent burial» (Gage).

Décent au sens de «belle, bonne, bien meublée [d'une maison]" est également un emprunt sémantique à l'anglais : «meeting at least the minimum standards of quality, etc.» (Gage).

Décent avec l'acception de «vêtu bien proprement» vient du sens anglais "properly dressed to be seen in public" (Gage; OED).

Décent, «poli», vient du sens anglais «kind, accommodating, pleasant» (OED).

désappointer (Ms. 1 Cahier A; Ms. 2); «[tromper qqn dans son attente ou dans ses espérances]». Surtout employé au passif. Attesté au figuré depuis Cotgrave 1611. Il disparait au milieu du XVII ${ }^{e}$ s. et est repris en 1761 par Voltaire à l'anglais to disappoint (lui-même emprunté anciennement au français). Le mot dans cette acception n'est pas enregistré comme un anglicisme dans les dictionnaires du XIXe s. et du début du $\mathrm{XX}^{\mathrm{e}}$ s. (Bescherelle 1858; Littré; DG le donne comme un néologisme). "Désappointé, qui a été critiqué en français au début $\mathrm{du} \mathrm{XIX}^{\mathrm{c}} \mathrm{s}$., s'est répandu dans l'usage sans que sa formation et son rattachement sémantique soient clairs pour le locuteur» (ReyHist sous point). 
dirigé (Ms. 1 Cahier A; Ms. 2); être dirigé de, «être enjoint de, avoir ordre de». De l'anglais to direct, «to request or enjoin with authority» (Webster; Gage); à la forme passive : "I was directed to» (GHarrap). Kœessler (sous direct (to) : «donner des instructions, des ordres à»).

dram (Ms. 1 Cahier A); «train de bois flottés». Mot anglo-canadien qui désigne «a section of a timber raft, made up of several cribs lashed together» (Gage et DictCan, qui le donnent comme un terme appartenant de nos jours à l'histoire du flottage du bois). Consigné dans Maguire, Clapin («La fraction la plus considérable d'un train de bois, ou cage [...]") et Dionne (sous drame).

finir avec (Ms. 1 Cahier B); «n'avoir plus besoin, ou achever, terminer et simplement finir». De l'anglais to finish with, «finish using; come to the end of one's need of: Have you finished with my book yet? (Gage). Relevé dans Dagenais (sous prépositions (emploi des ) 414).

game (-coq) (Ms. 1 Cahier B); «[mâle élevé et entraîné pour les combats de coqs]». De l'anglais game-cock, «a rooster bred and trained for cockfighting» (Gage; Webster).

hausse (Ms. 1 Cahier A; Ms. 2); «sorte de vêtement en usage chez nos Sauvages, qui sert à couvrir la jambe». De l'anglais hose, "a cloth leg covering that sometimes covers the foot» (Webster). Hose est employé, comme terme générique, en 1763, dans un exemple anglais, qui explique le mot mitasse : «a kind of hose» (DictCan sous mitashes or mitasses). Le mot heuse au sens de «botte, guêtre», qui a vécu en ancien français et qui a été relevé en Normandie, n'est probablement pas à retenir ici comme origine de bausse (FEW 16, 228a sous bosa). Consigné dans Dunn, Clapin et Dionne (sous hausses), Glossaire (sous bausse). Recueilli en Acadie (Massignon 1670). V. Mitasse.

immatériel (Ms. 1 Cahier A; Ms. 2); «léger, peu grave». De l'anglais immaterial "of no substantial consequence : unimportant» (Gage; Webster; Kœssler : «On verra que material signifie important, essentiel; immaterial est donc sans importance»). V. Matériel.

improuver (Ms. 1 Cahier A; Ms. 2); «faire des progrès, se perfectionner; s'améliorer». De l'anglais to improve "make better; become better» (Gage; Webster; Koessler : "ne pas confondre to improve, improuver et to improve, mettre à profit, faire valoir, amener à un état plus profitable, cultiver (ses relations), améliorer, perfectionner").

Le verbe français improuver a signifié "réfuter (une opinion)" au XIV ${ }^{\mathrm{e}} \mathrm{s}$, "contredire» au XVe s., «blâmer, désapprouver» au XVI $\mathrm{XV}^{\mathrm{e}}$ s. ; «il est quasiment sorti d'usage» (ReyHist). Absent des dictionnaires usuels. 
inconsistant (Ms. 1 Cahier A; Ms. 2); «incompatible, contraire, contradictoire; inconsidéré». Emprunt à l'anglais inconsistent, «not compatible with another fact or claim; containing incompatible elements; incoherent or illogical in thought or actions» (Webster; Koessler sous inconsistency, inconsistent). Consigné dans Dunn, Clapin et Dionne.

major, ore (Ms. 1 Cahier B) ; «majeur». Forme empruntée à l'anglais. L'adjectif est donné par Viger également dans tierce majore pour tierce majeure. Major a été relevé comme subst. masc. dans Clapin et Dionne ("Jeu de cartes, dans lequel le roi d'atout joue le rôle principal»).

matériel (Ms. 1 Cahier B); «de grande conséquence, important, essentiel». De l'anglais material, «having real importance or great consequences» (Webster; Koessler sous material). Consigné dans Glossaire; DavLang (sous material). V. Immatériel.

obligé (Ms. 1 Cahier A); être obligé à qqn pour qqch. Formule de politesse calquée sur l'anglais : "I am much obliged to you for your kindness" (GHarrap).

office (Ms. 1 Cahier A; Ms. 2); "[bureau, étude, cabinet de travail (d'un notaire, d'un avocat, d'un médecin, d'un homme d'affaires, d'un marchand, etc.)]». De l'anglais office, "a place in which the functions (as consulting, record keeping, clerical work) of a public officer are performed; the directing headquarters of an enterprise or organization; the place in which a professional man (as a physician or lawyer) conducts his professional business» (Webster; Gage). Consigné dans Dunn, Clapin, Dionne et Glossaire. Encore en usage au sens de "réception, bureau»; emploi critiqué (DQA). V. également les sens français du mot office dans la partie Archaismes.

ordonné (être de) (Ms. 1 Cahier A; Ms. 2); «avoir ordre de». Emprunt sémantique à l'anglais to order, «to command to go or come to a specified place» (Webster).

originer (Ms. 1 Cahier A; Ms. 2); «provenir, tirer son origine ou son étymologie, dériver». Forme francisée du mot anglais to originate, "to take or have origin : begin» (Webster). Cependant, en français général, le verbe originer issu du terme origine est attesté sporadiquement à différentes époques. Attesté une première fois en moyen français : originer, «créer pour»; originé de, «qui tire son origine de», attesté à la fin du XVe s. Repris en 1791 : originer de, "provenir de" (FEW 7, 416a sous origo). Le verbe est réintroduit en français moderne, à la forme pronominale : s'originer, «faire remonter son origine à» (vers 1968) (TLF et ReyHist sous origine). Semble peu usité; il est absent des dictionnaires usuels. Relevé dans DavLang (sous originate (to)), Dagenais (sous origine) et DarbDict. 
payer (Ms. 1 Cahier A; Ms. 2); payer une visite, ses compliments, ses respects "faire, rendre». De l'anglais to pay, «give; make; offer : to pay compliments, to pay a visit» (Gage). «To pay a un sens beaucoup plus large que payer: l'angl. l'emploie là où nous avons tendance à user du verbe faire, qu'il s'agisse de faire sa cour, un compliment, une visite, attention» (Koessler sous Pay (to)). Consigné dans Dunn, Clapin, Dionne, Glossaire et Dagenais. Encore usité dans certains milieux en contact avec l'anglais.

plated (Ms. 1 Cahier B); «fourré, ée [«plaqué, ée»], pour les vases et autres meubles de cuivre dorés ou argentés d'un côté». En anglais : plated "covered or overlaid with a thin film of gold or silver. Also used with reference to metals other than gold and silver» (OED).

L'adjectif fourré, au sens de "recouvert d'or ou d'argent", est attesté en orfevrerie depuis 1464, mais il est vieux de nos jours (ReyHist sous fourrer; PRob 1993). V. le sens de meuble qui figure dans la définition dans la partie Archaïsmes.

retraiter (Ms. 1 Cahier A; Ms. 2); «faire retraite, se retirer [d'une troupe]». De l'anglais to retreat, "to retire before superior force or after a defeat (of an army or a combatant)» (OED). Retraiter est attesté, en français, au $\mathrm{XV}^{\mathrm{e}}$ s., "retirer une troupe», et au $\mathrm{XVI}^{\mathrm{e}}$ s., "se retirer, disparaître» (FEW 10, 342a sous retrabere). Retraiter, au sens de «se retirer (d'une troupe)», semble peu usité en français; frgure dans TLF, qui donne un exemple d'emploi datant de 1931.

rôle (Ms. 1 Cahier A); «petit pain». De l'anglais roll, «a small piece of dough which is cut, shaped, and often doubled or rolled over and then baked» (Gage). Dionne enregistre le mot au sens de «gâteau au beurre, sans sucre" (sous rôlle).

style (Ms. 1 Cahier A); dans le (grand, baut) style, «du meilleur goût, de la dernière mode». Pris en ce sens, style est un anglicisme : «a fashionable, elegant, or admirable way or manner : She dresses in style» (Gage; Koessler). Consigné dans Glossaire.

\section{DATATIONS}

Avec toute la prudence que commande ce genre de compilation, nous avons relevé dans le travail de Viger un certain nombre de mots et de sens qui peuvent être considérés comme des premières attestations. Comme le fait remarquer à juste titre P. Rézeau dans Le «Vocabulaire poitevin» : «[II] faut évidemment $y$ voir un jalon qui demain sera dépassé.» 
abat : de grands abâts de pluie, «fortes pluies», est attesté en français depuis 1863 (FEW 24/1, 17b sous abbattuere).

ahurissant : cette personne est bien aburissante, «ennuyeuse». L'adjectif aburissant est consigné dans les dictionnaires français depuis 1866, d'après Lexis et GLLF.

allumer : entrons allumer, «mettre le feu au tabac d'une pipe». Allumer une pipe n'a été relevé qu'en 1845 (FEW 24, 340a; ReyHist).

baiser : il a été baisé dans telle occasion, «trompé, attrapé». Ce sens a été relevé en 1871 dans MartVend; en 1881 comme terme argotique (TLF).

blonde : «amante [jeune fille courtisée; fiancée]». Figure dans les dictionnaires français depuis Boiste 1829.

boudin (faire du ) : il fait du boudin pour dire d'une personne qu'elle boude. L'expression est consignée dans Bescherelle 1858.

bouquin : «c'est ou une plume teinte, ou un petit cilindre, percé aux extrémités, [...] dont nos habitans se servent pour orner leurs pipes, ou en alonger ce qu'ils appellent le manche». Ce sens est attesté depuis 1833, d'après TLF et ReyHist sous bouche.

canoter : «conduire un canot» et «se promener en canot». Le sens que donnent les dictionnaires est celui de "se promener, pratiquer la promenade en canotm. Il est attesté depuis 1858, d'après TLF et ReyHist.

écœurant : «malpropre». Attesté depuis 1870 (FEW 2/2, 1172b).

écœurer : «faire soulever le cœur, faire mal au cœur». Attesté en français général depuis 1864 (FEW 2/2, 1172b; ReyHist sous cceur).

escabeau : «on appelle ainsi un meuble à plusieurs degrés, portatif, dont on se sert dans la maison, pour atteindre à quelque chose d'élevé, en montant dessus». Cette acception est attestée depuis 1875, selon TLF.

flambant : on dit d'une personne babillée tout en neuf, qu'elle est flambante. Dans ce sens le mot est attesté depuis 1837 (FEW 3, 603b; ReyHist sous flamber).

garde-corps : «balustres que l'on met au bord des ponts, des quais, etc.». Dans ce sens que relève GLLF : «barrière établie le long du tablier d'un pont, le long d'une terrasse, d'un lieu élevé pour empêcher les passants de tomber», le mot est daté de 1872.

loucheur, euse, subst. : je ne veux point d'une loucheuse. Un loucheur. Le substantif loucheur, euse, "personne qui louche», est attesté depuis 1823 (ReyHist sous louche). 
loucheur, adj. : cet homme est loucheur. L'emploi adjectival n'est attesté en français général que depuis 1936. Il est rare d'après ReyHist sous louche.

mèche : "extrémité la plus déliée d'un fouet, et qui souvent est une ficelle rapportée». Attesté depuis Académie 1835 (FEW 6/3, 323a; TLF; ReyHist).

pelleter : «remuer, jeter avec une pelle». Attesté sous l'ancienne forme peltrer en 1776; la forme pelleter est attestée en français général depuis 1845 (FEW 7, 481a; ReyHist sous pelle).

péter : Je crains qu'il en piete. Au sens de «mourir», péter est attesté depuis Flaubert 1844 (FEW 8, 133b).

train (en ) : «ivre». Attesté en français général depuis 1872 (FEW 13/2, 164b).

voyage : «charge transportée». Attesté depuis 1872 (ReyHist). 


\section{APPENDICES}

Les documents que nous présentons dans cette partie contiennent des listes de mots et d'expressions dressées par Jacques Viger lui-même et par le député-poète Ross Cuthbert. Ce dernier a fourni à Viger un nombre considérable de mots et d'expressions. Viger a largement puisé dans ces listes pour établir sa nomenclature; l'ajout de nombreux signes, les phrases raturées, les précisions ajoutées montrent, de toute évidence, qu'il a travaillé en s'aidant de ces listes. Les mots et les expressions qu'elles contiennent constituent la base de son travail. 


\section{Fo.Verreau $67 \quad$ NO}

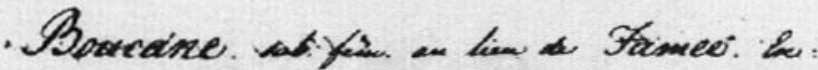

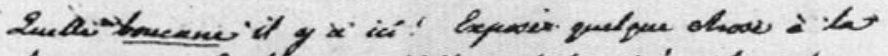

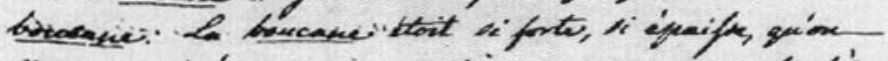

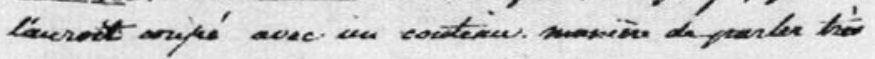
cosinercenester.

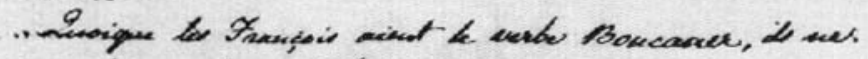

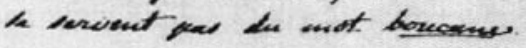

ircbives da Sémlàalro de guabsc

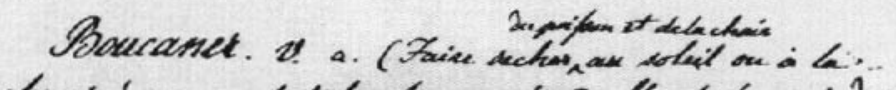

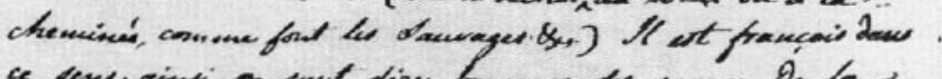

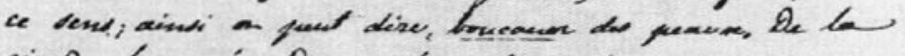

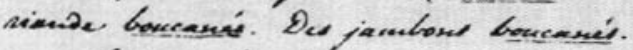

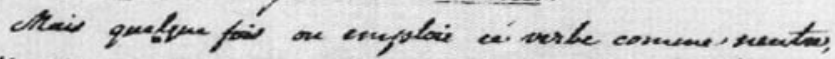

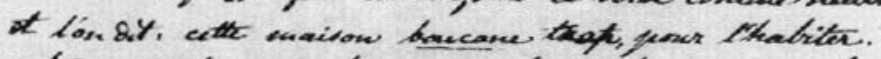

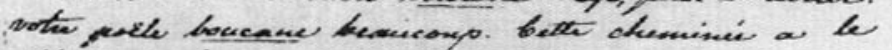

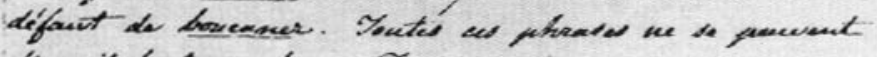

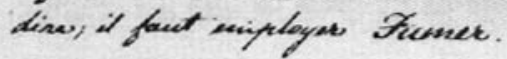

Les articles Boucane et Boucaner du manuscrit 2 de la Néologie canadienne de Jacques Viger. Manuscrit conservé au Musée de la civilisation, fonds d'archives du Séminaire de Québec. 


\section{LISTES DES MOTS ET DES EXPRESSIONS RELEVÉS PAR JACQUES VIGER}

\section{DOCUMENT I}

(de la main de Jacques Viger)

\section{Proverbes, \& autres manières de parler en usage.}

Le vent se tourne du côté des mitaines. - Il se met au froid.

On peut ramer sans culottes.

C'est trop de valeur.

Ç'à fait trembler, - çà fait peur.

C'est comme le moulin de La Chine, qui ne s'arrête plus quand il est parti.

A go-et-gon.

Il a un oeil à la coque et l'autre à la mouillette, pour louche des deux yeux.

Il a la tête comme un procès-verbal.

ni quoi - ni qu'est-ce.

chat échaudé craint l'eau froide.

manger à la croc $[$ sic $]$ au sel ${ }^{(1)}$.

manger de l'avoine - couper l'herbe sous le pied.

[manger] des guêpes.

leste comme un chien de plomb.

ric-à-rac.

cracher sur les tisons.

il a ${ }^{(2)}$ sot, comme un fondeur de cloches.

courir les côtes.

Il a perdu un pain sur sa fournée.

Les pieds lui brulent.

Il a bien des croutes à manger.

c'est un bout d'homme.

à preuve (adverbe).

Il n'a pas la tête aux pieds.

mettre la charrue devant les bœufs.

à moi le mur.

il branle dans le manche.

Le lard sera à bon marché, les cochons s'embrassent. - (de 2 hommes qui s'embrassent).

Je ne suis pas la servante du curé.

Ça le remêt sur farine.

prendre martre pour Renard.

fumer, (sinonime de corner \& biner).

une suerie. - abriller - s'abriller. 


\section{DOCUMENT II}

(de la main de Jacques Viger)

Pour établir cette liste de mots, Viger s'est inspiré de l'ouvrage d'Alexander Henry, Travels and Adventures in Canada and the Indian Territories (v. bibliogr.).

\section{Histoire naturelle.}

\section{Noms Canadiens.}

Pierre-à-calumet (compact lime-stone).

Masquinongé (a specie of Pike ou Brochet).

Caribou (par les Sauvages - O'tic, A'tic) Le Renne.

Le Liard (populus nigra, - poplar-tree).

Oiseau blanc (Emberiza hyemalis).

Poisson doré (pickerel, petit Brochet).

Orignal (cervus alces, - moose-deer) Elan.

Pantère, Tigre \& plutôt Couguar $\}$ Felis concolor).

Perdrix - This name is given to more than one species of grouse. The birds here intended, are red grouse.

Loup-Cervier ou Lynx.

Carcajou ou Glouton.

Maringouin.

Moustic.

Fenouillette ou Fenouil.

Foin sauvage.

Herbe à la puce.

Herbe à Dinde.

Matelot.

Otocas.

Souris-chaude.

Tondre substantif.

Achigan.

Epinette ou Arbre de vie ou du paradis.

Passe rose ou Mauve des Jardins.

Poisson blanc.

Bleuet. 


\section{DOCUMENT III}

(de la main de Jacques Viger)

La majorité des termes ainsi que le texte qui suit proviennent également de l'ouvrage précédemment cité d'Alexander Henry. Les extraits que Viger a retenus sont séparés dans son texte par des tirets et se trouvent aux pages $13-14 ; 14 ; 15 ; 23-24 ; 24$ en note $; 30 ; 53$ en note $; 78$.

\section{Voyages au Haut-Canada}

Voyageur - canot d'écorce - guide - camper - wattap - gum varengues - brigade - bouts-de-canot - piéces - portage - décharge - pierre à calumet - masquinongé - caribou (par les Sauvages - O'tic, a'tic) - Le cèdre (thuya occidentalis) - hyvernement - lait (pour rum) - perdrix - coureur des bois - Gens de terres (têtes de boule) ${ }^{(3)}$ - mal de raquette - jeu de la crosse - awapou - apichimon - le Liard (populus nigra, poplar-tree) washes - faire chaudière - faire de l'eau - Indian path - Emberiza hyemalis (oiseau blanc) - tripe de roche (Waac) - folle avoine, riz sauvage (avena fatua, zizania aquatica) - rapide - poisson doré (pickerel) - Elan (moosedeer, cervus alces) - praline - pantère, tigre, $\&$ plutôt couguar (felis concolor) - esclave - stroud blanket - Bastonnois - Sacacoua - équipement.

The canoes, which I provided for my undertaking, were, as is usual, five fathom \& $1 / 2$ in length, and $41 / 2$ feet in their extreme breadth, and formed of birch-tree bark, a quarter of an inch in thickness. The bark is lined with small splints of cedar-wood; and the vessel is further strengthened with ribs of the same wood, of which the two ends are fastened to the gunwales : several bars, rather than seats, are also laid across the canoe, from gunwale to gunwale. The small roots of the spruce-tree afford the wattap, with which the bark is sewed; and the gum of the pine-tree supplies the place of tar and oakum. Bark, somme [sic] spare wattap \& gum, are always carried in each canoe, for the repairs which frequently become necessary. - To every 3 or 4 canoes, which constitute a brigade, there is a guide or conductor. - The freight of a canoe... consists in 60 piéces, or packages, of merchandise, of the weight of from 90 to a 100 pounds each; $\&$ provisions to the amount of 1000 pounds. - We now reached the channels of the grand Calumet, which lie amid numerous islands, and are about 20 miles in length. In this distance, there are 4 carrying-places (portage) besides 3 or 4 décharges, or discharges, which are places where the merchandize $[s i c]$ only is carried, \& are therefore distinguishable from portages, or carrying-places, where the canoe itself is taken out of the water, \& transported on men's shoulders. - The pierre à calumet is a compact lime-stone, yielding easily to the knife, $\&$ therefore employed for the bowls (fourneau) of tobacco-pipes, both by the Indians \& Canadians. - 
Among the pike, is to be included the species called, by the Indians, maskinongé. - In North América [sic], there is no partridges; but the name is given to more than one species of grouse. The birds here intended, are red grouse. - Baggatiway, called by the Canadians le jeu de la crosse, is played with a bat and ball. The bat is about 4 feet in length, curved, \& terminated in a sort of racket. Two posts are planted in the ground, at a considerable distance from each other, as a mile or more. Each party has its post, and the game consists in throwing the ball up to the post of the adversary. The ball, at the beginning, is placed in the middle of the course, $\&$ each party endeavours as well to throw the ball out of the direction of its own post, as into that of the adversary's.

\section{DOCUMENT IV}

Liste de mots dressée par Viger et dans laquelle il a puisé pour établir sa nomenclature.

travail, menoir

guides, corrois

cordeaux, courroies

canard, bombe

faire coup

office

appointer

désappointer

opportunité

confortable

incliner

guide en fait de voyageur

amarrer

à mont la côte

la relevée

payer visite

paré pour prêt

la sentinelle - factionnaire

un incendie

chardron

obligé, obligation

garde-corps avisse pour vis

boucane

demancher

amancher, emmancher

ramancher

embarquer \& débarquer

tuer la chandelle

allumer, charger

manche d'une poile

couette

cariole, traineau

traine $\&$ traine de clisse

cabriolet, cabrouet

brancard

boyard

bran de scie

berlancille pour brandilloire

berlanciller pour se brandiller

trompe - instrument - il est

françois

gouette

butin 


\begin{tabular}{ll} 
stile & côte \\
décent & cheniquer \\
robe de bœuf & jument \\
caribou & âbre \\
orignal & frét, fréde \\
chat sauvage & ahurissant \\
bouquin & mitaine \\
cadre & mitasse, hausse \\
improuver & tuque, mâle \\
retraiter & matelat \\
voyage d'eau & mouiller \\
scieau & sorcière \\
argent & espérez-moi \\
retrayer & berre \\
balayer & mèche \\
frasil & mèche : mesure \\
frimat & marier quelqu'un \\
poudrerie & araignée fém. \\
tirans & fenouil masc. \\
bolle & fart ou farce \\
faucille & berloque \\
crocheter & brimbale \\
tambour & escousse \\
rigole & papier cassé \\
tondre & papier brouillard \\
empois & notureau \\
pesâ & taure, tauraille \\
amidon & licher \\
buffet & tire \\
houiller & orge mondée \\
plairie & resous de résoudre \\
couronel & pelote, paume \\
corporal & raquette \\
cartron & matelot, insecte \\
pipe : mesure & loucher, bicler \\
bourg[u]ignon & mouillette \\
bordée & grouiller \\
onglée & écroi \\
\hline & \\
\hline &
\end{tabular}


çà fait trembler :

çà fait peur

c'est trop de valeur

chez nous

étage

diriger

remettre

banal

étal

celle-ci

celle-là

deusse, troisse

treffle

geremium

j'ai arrêté une maison, un habit, des bottes

affaire matériel

cas matériel

superbe

embarrasser

oubli

bord

micmac

brin

casseau

papier nouvelles

papier tenture - tapisserie

cornichon

escabeau

pucelage

porchais

pourceline

pouliche

ouette

épiochon

micoine

Juive

bougon balle \& paille

boudin

berdâs, berdasser

remue-ménage

souliers françois

souliers de bœuf

apichimon

prouvable

rustique

niveleux

suspect

malin

vulgaire

chance, chanceux

çà te froise

emberlicoter

batailleur

gourdin

fouillouses

écopeau

chaume

bandon

capuche

lévier

mitonner - choyer

train - dépense, ivre

biner, corner, dépit

vire-l'œil

écœurer

borgne

verglas

couche chaude

chauve-souris

bourasser

ençà

brouscailler

tricoler

se fier 


$\begin{array}{ll}\text { rubrique } & \text { haut mal } \\ \text { malin basque } & \text { mal de Saint } \\ \text { escolter } & \text { mal de saint Jean } \\ \text { dresser } & \text { épilepsie } \\ \text { dévider } & \text { quêteur } \\ \text { crible, train } & \text { tamis } \\ \text { marinage } & \text { nix, nix for stein } \\ \text { fine-boutique } & \text { je suis ordonné de } \\ \text { driller } & \text { empêtrer } \\ \text { tapon } & \text { pelleter } \\ \text { retailles } & \text { du mil - foin } \\ \text { étriver } & \text { décaniller } \\ \text { tout plein } & \text { fictif, ive, adj. } \\ \text { bête-puante } & \text { sûrir } \\ \text { portépis } & \text { graissoux, se } \\ \text { débrailler } & \text { verbalement } \\ \text { devergondé } & \text { glumer } \\ \text { ebrailler } & \text { warrant } \\ \text { attends-toi-zy } & \text { bill } \\ \text { rappelle-toi-z-en } & \text { indictement } \\ \text { brayer du lin } & \text { indicter } \\ \text { fener le foin } & \text { râle, râler } \\ \text { veilloche, mulon } & \text { ébarouir } \\ \text { belt, bandoulière } & \text { éclopé } \\ \text { retontir } & \text { galimafrée } \\ \text { tinton } & \text { jouqué } \\ \text { originer } & \text { aveindez-le } \\ \text { délivrer les lettres } & \text { dégrader } \\ \text { c'est un game } & \text { ebourifler } \\ \text { un game coq } & \text { culasse } \\ \text { conviens-toi-z-en } & \text { Guide - longue rêne attachée à la } \\ \text { donne-moi-z-en } & \text { bride d'un cheval attelé. } \\ \text { plated, fourré } & \text { Rêne - courroie de la bride d'un } \\ \text { fourreur - pelletier } & \text { cheval. } \\ \text { butard } & \text { Cordeau - corde de moyenne } \\ \text { barbeau, insecte } & \text { longsseur. étroite et qui sert à lier, à } \\ \text { criquet } & \text { attacher quelque chose. } \\ \text { tomber d'un mal } & \end{array}$




\section{LISTES DES MOTS ET DES EXPRESSIONS RELEVÉS PAR ROSS CUTHBERT \\ DOCUMENT V \\ (de la main de Ross Cuthbert)}

Notte pour Jacques Viger - Esquire

Epigraphe de ton Dictionaire

Athenes tu as fini, Rome tu vas périr

L'Ecole Canadienne, dissipe ton Souvenir

Extrait de... L'Aréopage

par le Square Ross Cuthbert

[Col. A]

tu pars comme un fusil sans plaque

il chante comme un rêve

il leve le c... comme une bête puante

il marche comme un chien qui vient des vêpres

un beau canard!!

c'est son père tout racopié

il braille comme un veau

haut comme un choux [sic]

ils en font un patira

c'est comme baise mon pouce

c'est un valtreux

J'aime cela comme la colique

Ecoutes [sic] donc, mon fiston

J'ai déboulé en bas de la cote

Je me suis agrippé ${ }^{(4)}$ après les branches

Je marchois après lui / pour derriere

courir au busc

déplanter un oiseau, \&c.

un cheval qui coure [sic] le gallop [sic]

manger de la sagamité

il bègue / pour begaye

Je ne bèguerai pas, pour te dire cela 
c'est de l'Etaim [sic] fine

il est mal à main

c'est un homme factieux / pour facécieux [sic]

il m'a donné des mauvaises raisons / pour il m’a mal reçu, \&cc.

c'est une raison ça

impotheques, déventes

Les gros chiens ne se mordent pas

il a été presque, presquement / pour preste

c'est long comme d'icite à demain

Je l'ai tournaillé, travaillé

ç'a fait grincher les dents

ça ne portera pas a terre / pour relever

vite, pendant que c'est chaud / vitement

Je me suis Estropié

\section{[Col. B]}

essayer à manger

c'est tout de bon, pour serieusement

c'est un chétif

il est dur comme un cheval

donner le bois ${ }^{(5)}$ pour les Ecopaux

il est jouqué comme un dinde

poulailler

il est Elingué. Etiré

c'est usé à forfaite

régal, je me suis régalé

Les bouchers donnent trop de régal

il a éparpillé cela

Il a les yeux à la perdition de son âme

il a les jambes comme des batons de traine

cet homme a un beau paroli

il est bien induqué

il n'est pas mechant / pour exprimer qu'il a quelque talent

c'est du bois de requise, pour de valeur

bavasser

pietes $[$ sic $]$ toi

rire jaune 
Quelle est sa vocation?

barbots

faire des barbots

ça file dru, je filois doux

ça coule comme un sas / pour tamis

sasser

passer au gros sas

tout plein, il est fin tout plein

il empeste, il empoisonne l'ail, l'oignon

c'est de la poison

c'est de la carotte à Moreau

on doit plus à sa peau, qu'à sa chemise (françois)

chipoter (je le crois françois)

Je suis plein d'eau

un couteau plein de graisse

c'a fait trembler

chacun à son tour comme au moulin

il y a belle lurette

crocs pour moustache et pour dents

\section{[Col. A]}

il est fait au moule / pour au tour

[il est fait] en peinture / pour à peindre

il est ostiné

c'est un quêteux

avez vous des oublies pour cacheter

J'ai tout désoublier [sic], pour oublier

il courre [sic] comme un chat sauvage

cette affaire est bâclée

il y avoit beaucoup de roulins

de beaux cadres

fourgonner

elle s'est levée le derrière le premier

bougonner / renoter

Je l'eus bètot mis à l'envers

il a une mine à cracher dessus

gras comme un voleur 
Je ne sais de quel bord me revirer tâches [sic] donc de l'appâter c'est une dévergondée il a pris une belle embardée renchausser des patates il s'en va du coté de tantòt un petit rabougris la rente de l'argent / pour l'interet un couteau ébrèché (françois) le mal passe net comme sur la main étriper quelqu'un faire ètriver donnes [sic] la bouette à la vache fou comme braque une brique de savon (françois peut etre) il fait le chien couchant Je suis capable de manger cet homme là il se frôloit contre lui c'est jour de mitasse à cause donc (canadien pour le certain) Moyennement sa femme porte la culotte comme elle s'est attiffée il est changé / pour pâle, défait

\section{[Col. B]}

Je me suis mis dans mon lit mettre ses bas dans ses jambes [mettre] ses souliers dans ses [pieds] ${ }^{(6)}$ piler sur quelqu'un

l'argent sous le pouce parler verbalement à quelqu'un astigoiner c'a n'est pas de la petite biere mirlifichures / pour colifichets il a fait une sottise de brasse il est malin comme sept fois le Diable 
cé n'est pas le Diable / ce n'est pas difficile ça tombe comme la pauvreté sur le monde malade comme un chien cela prend au nez comme la moutarde parler comme une femme est un proverbe de tous les pays une langue à percer quatre murailles il ne fit frime de rien se plaindre le ventre plein il geint, geignoit, geindre donner une paire de soufflets boire à la grande tasse nager à grande eau

La bête à la grande queue cristail un etau / pour ètoc qu'est ce que vous grattez par ici Je lui ai parlé françois secquement, il m'a reçu secquement passer en belette une gaffe, gaffer quelqu'un il a les quatre pieds blancs / il est libre fin-fin la blague cest fait dans un vire-main apres moi le déluge hardi donc / pour courage

\section{[Col. A]}

cela me chiffonne

Je lui ai coupè le sifflet

il se promene les mains dans ses poches

faire une attisée (françois)

ils sont amis jusques au plat

il s'est dèboutonné (françois)

Jouer, boire, manger \&c. son saoul \& Content a refection propre comme un sou salopries [sic], pour saletés, ordures \&c. la flambe / de flamber (françois) 
lamper / lampas (franfois)

le reveil matin

l'herbe à la puce

l'herbe à dinde

il est comme l'oiseau sur la branche

a brousse Poil

Docteur en souppe salée

un habit rapiesté

il faisoit brun - à la brûnante - entre chien \& loup

il chante comme un perdu

crier comme un sourd (françois)

un homme mal équippé [sic], pour blessé

un homme saoul, se souler pour enivrer (francois)

Il fait la pluie et le beau temps

courir les Côtes, pour les Campagnes

fripper quelqu'un

il s'est epouffé pour échappé (francois)

c'est un mal a droit - drette

a cor et a cri

sans vous Interrompre

curieux comme la chèvre a Jacques Cartier

c'a n'a pas de rime

tuer roide comme une tourte

tomber comme une tourte

un goret

[Col. B]

Je suis affairé (françois)

un rin de vent / rhumb

une risée de vent

Piroli

tout flambant neu ou nu

soupirail (franfois)

des fanals, confessionals

elle est legerte

tout fin seul, (quelque fois faux)

ricaner (franf̧ois) 
il fait chaud que le Diable

il alloit vite que le Diable

mon petit gat pour gars

ferlasser (françois)

comment t'appelles-tu? pour se nommer (françois)

\section{[Col. A]}

J'ai travaillé une escousse

pivac pour bivouac

pique-bois

il a mangé à se mettre le ventre sur le dos

elle étoit sur un tata terrible

c'est bonnet blanc, et blanc bonnet

où avez vous pêché cela

un gaband

fendre des éclats

il a l'ame clouée, rivée dans le corps

on dit ici quelouer pour clouer

si la mer bouilloit \&c.

cela bouille pour boût

c'est pour le sur / pour certain

$s$ 'ingerer de quelque chose

tout cela fait bouillir la marmite

il ne pourra jamais ressoudre

bouler quelqu'un

Je te garde un chien de ma chienne

Je l'ai dégradé / pour laisser en arriere

il est degradé / pour il est arrêté

Je l'ai retappé

il avoit une poignée d'argent blanche

Je l'ai semée

tarabusquer

le temps est mucre

tu viendras cuire à mon four

ni plus, ni moins / pour cependant 


\section{DOCUMENT VI}

(de la main de Ross Cuthbert)

\section{Anglicismes et Expressions non françoises usitées par les Canadiens, Proverbes, \&c. \&c.}

\section{Notte}

\section{[Col. A]}

travail de charrette

amancher, demancher, ramancher

on dit enmanché d'un long col ${ }^{(7)}$

par exemple (Quand [1'] employé)

embarquer, debarquer

chat échaudé craint l'eau froide

Je vous serois obligé pour \&c.

Je vous remercierois pour \&c.

Je voudrois que cela seroit

marier quelqu'un

vous me tannez

Je suis ahuri

Je sors d'entrer

fais donc un apichimon

là-bas sur la montagne

Il y a ben du train ici

tues $[$ sic] la chandelle

Brasses [sic] donc le poële

sortir dehors

Manger des guêpes

Manger de l'avoine

il fait le faro

poudrerie

traine, cariole

guides, pour rênes

improuver au lieu de se perfection[ner]

desappointer pour tromper

Il s'est fait baiser en pincette 
payer une visite, des compliments

c'est bien de valeur

Je serois bien marri, faché de

voyons voir

cahots

allumons

c'est comme une mitaine sans pouce

Il en faut autant que de pelotte de neige ${ }^{(8)}$ pour chauffer un four

fier comme un Ecossois

c'est comme une troisieme roue à une charrette

il boit comme un trou

La poste à Saupin, de chien

faire le bredas, bredasser

la race des gueux ne se perdra pas il n'y a pas mortalité de canaille

\section{[Col. B]}

il a été reçu comme un chien à vêpres ou dans un jeu de quilles

pauvre comme un rat d'église

il ment comme un arracheur de dents

crocheter des pois

du pesas

La chienne à Cantin lui monte sur le dos

Bouquin pour les pipes

Boire une larme, un filet

Savant comme un livre. Pourquoi

ne diroit on pas, sot comme un livre?

il parle comme saint Paul, la Bouche \&c.

esperez moi un instant

mitonner, douilleter quelqu'un

ne me brousquaille pas tant

Je te flanquerai un tapin par les babines

il a mouillé toute la journée

Je suis tout trempe

Je suis trempe comme une souppe

tremper la souppe

renflé comme une souppe au lait

un canard, une bombe 
une bombarde

parler du nez

il est fierement riche

c'est une Juiffresse

Je suis démonté

elle est blême comme un linge ou encore comme... autre chose gueusasse

un sciau

un banc-lit

il n'est pas la moindrement fatigué

Les chemins unis comme des chemins de carte [sic]

rafistoler, rapapillotter

il fait un temps a ne pas mettre les chiens dehors

ça entre par une oreille et ça sort par l'autre

qui a fait Lundi, a fait Mardi

leste comme un chien de plomb

il est connu comme Barrabbas a la passion

\section{[Col. A]}

tu ne vaux pas les quatre fers d'un chien

donnes [sic] moi ma tuque, ma capuche

il a une tête d'allemand

acheter des souliers de bœuf, des souliers françois et Sauvages

donnes [sic] moi ma bougrine

ils m'ont emberlicotté

Je me suis blousé

flâner, flasque, flandrin, lambin, lambiner

mais c'est que (à tout propos)

couette, gouette

un habitant, pour agriculteur, paysan

c'est ecœurant

se trouver mal

vomir tripes et boyaux

il y a du train dans le bal

J'étois dans l'Inquilibre / doute, indecis

un voyage d'eau

le vin est sur (aigre) et lait sur 
farine du diable retourne en son

la tricherie retourne à son maitre

fumer sans pipe

Je te ferai passer par un chemin ou il n'y aura pas de pierres tous les bourdignons

il fait frette

mets tu ton col noir / cravatte

J'ai attrappé [sic] l'onglée

il tremble les fievres

faire chaudiere à part

Je te fricasserai une ramasse

c'est prouvable / c'est evident

c'est vulgaire / c'est clair

c'est un saint épais

il est grossier comme un pain d'orge

Je m'en vas sus ma tante / chez

c'est malin de plaider avec le seigneur

c'est un homme rustique

[Col. B]

vas donc ramasser des écopaux

voyez donc, ce petit nijon, bougon

il est amoureux comme une chatte

on n'attire pas les mouches avec du fiel

c'est une gavache

Je lui ai donné un savon d'importance

Je l'ai brassé comme il faut

c'est une buche

c'est un fin matois, une fine boutique

tiens bon, tiens fort, tire Dieu, tire Diable, Bougre à Bougre est [sic]-tu paré?

Dégobiller

renâcler

allez-vous cuiller les pommes?

Je te releverai du péché de paresse

faire du boudin

se mirer 
se dèbarbouiller

cet homme a de la corde de pendu

Je lui en ai donné sur les epicailles

il s'est grisé

descendre les escaliers quatre à quatre

Je ferai cela comme un des mieux

ils brulent la chandelle par les deux bouts

il a perdu un pain sur sa fournée

il grelotte

faites donc mortifier la viande, la salad[e]

vas dire à la Bejin qu'elle te peigne

c'est un mal peigné

Beurrer son pain

veux-tu une beurrée de graisse? \&c.

il est mal amanché dans ses affaires

il voudroit fignoler

cavalier (dans quel sens?)

Il va voir sa blonde

droit comme un piquet

faire manger de l'avoine a quelqu'un

[Col. A]

c'est un fier matin, un roustaut

c'est un vive la joie / sinonyme Roger bon temps

Je connois cela comme ma poche

donnes [sic] moi mon butin

courir la galipotte

renvoyer quelqu'un la pelle au \&x.

naturellement c'a fait ma [sic] plaisir

c'est une drolesse

quel ratapiat

aller a la cantine

pas tenseulement un sol

Je vais canoter

il a eu le pesant toute la nuit

une coppre

vous avez cochonné cela 
pourquoi gaspiller le butin comme ça

il s'amuse à brocanter

il est toujours à tripoter dans la boue

Je t'ecrapoutinerai le nez

c'est de l'onguent miton-mitaine

regarder quelqu'un par dessus l'épaule

Sec comme un coton ${ }^{(9)}$ de bled d'inde

- comme un manche de balai

- comme un pain d'épice

- comme Nord Est

c'est un batailleur

il mange comme un goulia

Sans demander ni quoi ni qu'est ce

aller tout de gô

il est fort comme un turc

il s'est éjarré

une traine de clisses

vois donc ce vire-l'œil, ce bicleux

veux tu fumer une touche?

des noix douces, amères

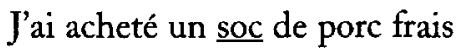

vous étiez a gogo

Nous avons fait ribotte / cela vient de ribauder

Turpin moine autrefois, puis archevêque et ribaud en tout temps. ribaud là, signifie a peu pres paillard.

voyez comme il fait le Monsieur. C'est comme un pou sur une galle

[Col. B]

c'est un homme du Nord

Eplucher

èplucher du bled-dinde

il lui a graffigné le visage

Balier pour balayer

apres qu'il a ballié, il n'est pas nécessaire d'épousseter

tomber dans la place

oh! le bagoulard

il a fait ses georges, pour orges 
desargenter, être désargenté

me voilà sur le plancher des vaches

[se] laisser manger la laine sur le dos

tête d'escargot - de boule

Et moi - je m'appellerai joli-cœur

Il a bien des croutes à manger

gober, ${ }^{(10)}$ des mouches

bâiller le bec

sec comme un pendu

faire de 5 sols 6 blancs

poches - empocheter

il ne vaut pas son plein cul d'eau chaude

corps vide comme un fanal

le petit bonhomme ou la vieille lui jette du sable dans les yeux

qui a la gale la gratte, qui ne l'a pas l'atrappe [sic]

bavasser

grichou - griche-poil

sur votre respect

à raz terre, tout a raz, razer

J'ai été mal équipé à ce combat

Je n'en ai pas pour une creuse dent

une belle ariette

Une beauté!

avoir de l'arse

sonner le tinton \& le tinton sonne

Brique de savon

tombé comme le gin-seng

tabac du diable (jusquiame)

petit lait (babeure [sic])

à brousse poil

batelée

tabaconiste pour tabagiste

Blague

être affairé 


\section{DOCUMENT VII}

(Notes de Viger, recopiées par quelqu'un d'autre.)

\section{acceptions canadiennes, ou provinciales}

Allumer : intransitif sous entend $u$ pipe : se reposer ${ }^{(11)}$; visiter; arrete allumer appointer : nommer à un poste. Monsieur a été appointé...

bombe pour canard : bouilloire. On dit plutot ${ }^{(12)}$ bombe dans [le] district de Québec plutot canard dans celui de Montréal

beurrée de crème, sirop

Capuche : bonnet de femme. Capuche de castor

mouiller : pleuvoir

jument : caisse de fusils

\section{Mots nouveaux}

Apichimon vient du sauvage : bourrelet de linge...; lit; grabat

Abât : bordée; orage, abât de pluie, de neige

boucane : fumée

chienneter $=$ chienner

flambant : neuf

\section{Notes des Appendices}

1. Phrase raturée.

2. Mot illisible.

3. têtes de boule ajouté au-dessus de Gens de terres.

4. r à la finale dans le ms.

5. la piece ajouté au-dessus de bois.

6. Écrit bieus.

7. Écrit verticalement dans la marge.

8. s final raturé.

9. sucet ajouté au-dessus de coton.

10. Mot illisible.

11. inviter raturé.

12. plutot ajouté au-dessus. 


\section{REGROUPEMENT ONOMASIOLOGIQUE}

1. L'atmosphère

Le temps et le vent

abât, bordée, giboulée; clairon, tirans; dégelée; fréde, fréte, fréte comme glace; mouiller; neige, pelote de neige; poudrer, poudrerie; rhimb de vent, sorcière.

2. La terre

L'eau

bourguignon; remous, tourniquet.

La flore

âbre; arbre de vie, arbre du paradis; bleuet; chardron; épinette; fenouil, fenouillette; ginseng; herbe à dinde; herbe à la puce; otocas; trèfe.

\section{La faune}

Quadrupèdes : caribou; orignal; porc-épic.

Mammifere volant : souris-chaude.

Oiseaux : oiseau blanc, ortolan; perdrix.

Poissons : achigan; maskinongé; poisson blanc; poisson doré.

Mollusques : pistolage, pucelage.

Arachnides et insectes : arignée; matelot.

3. Le temps et l'espace

après-dinée, relevée; faire brun, brunante, à la brunante, brune, sur la brune, entre chien et loup; arse, place «sol d'une pièce»; espérer; icit; mèche et pipe "mesure de distance»; sur «chez».

4. L'être physique

Le corps

couette "queue de cheveux»; regricher; reintier.

\section{L'apparence}

banal; beau comme un faro de campagne; bougon, petit gâ, nijon; écœurant (subst.), galeux comme un Écossois, graissoux (subst.); grichou, laid comme un grichou; tapon. 


\section{La vue}

bicler, bicleux, louche, loucher, loucheur, vire-l'œil; éborgner; stellà, stellci $(\mathrm{t})$.

\section{L'ouie}

gricher des dens; péter; retentir, retontir; sarpidon; tinter, tinton.

\section{Le sommeil}

dormir comme une soupe; pesant (subst.), avoir le pesant.

\section{Les indispositions, les maladies et les blessures}

dégobillage, dégobiller, dégobillis; écrapoutiller, écrapoutir; égrafignure, égratigner, égratignure, graffigner; équipage, équipé, équiper; gouette; épilepsie, haut-mal, mal caduc, mal de saint, mal de saint Jean, tomber d'un mal; ouète; soigner.

\section{L'alimentation}

beurrée, beurrer; boire du café comme la Brillant; castonade; envarié; fard; gouliâ, gouliafre; se houiller, être en train «ivre»; licher, s'en licher les barbes; marinade, marinages; naturel; poudine, poutine; rôle; soc; sur, surir; tapon de graisse; tire.

\section{Le tabac}

allumer; bougon de pipe; bouquin de pipe; charger; manche de pipe; fumer une touche.

\section{Les vêtements}

amarrer; berloque; butin; capuche, casque; ébrâillé, ébrâiller, écolté, s'écolter, épotraillé; ferlassement, ferlasser, flasque; flambant, flambant neuf, flambant nu; gaspiller; graissoux; hardes; hausse, mitasse; mâle, tuque; oreille de soulier, tiran de soulier; soulier de bœuf, soulier françois, soulier sauvage; grand, haut style; trempe, trempe comme une soupe.

\section{L'être intellectuel et affectif}

\section{Les sentiments}

ahurissant; baiser en pincettes "embrasser"; baiser "tromper", baiser en guedou et baiser en pincettes «tromper"; biner, corner; avoir, donner un beau bled d'Inde; faire du boudin, bourasse, bourasser, bourasseur, à (re)brousse-poil, à (re)griche-poil; bredasserie, bredassier; éviter une belle chandelle; cheniquer, cheniqueur, flasque, flasquer, gavache; désappointé, désappointer; étrivant, étriver; fine-boutique; flandrin, flâner; se fricasser de qqch.; grichou, malin, malin comme un Basque, malin comme un Cree, malin comme un griche-poil, malin comme un grichou; houillé de qqn, de qqch.; obliger pour, remercier pour; plaisant; rustique; suspect; tête d'Allemand, tête de boule, tête d'escargot. 


\section{L'élevage et la culture}

\section{Les animaux domestiques}

banal; chaton(n)er, chatter; chienner, chienneter; corner, ébrayer; écroi; game (-coq); se jouquer, jouquoir; notureau, porchais; tauraille, taure.

\section{La terre}

bandon, avoir bandon, donner bandon; côte; plairie.

\section{La fenaison et le jardinage}

bled; bled d'Inde, maï; braye, brayer; coton de bled d'Inde «tige», sucet; coton de bled d'Inde «épi dégarni», épiochon; couper, scier les bleds; crocheter des pois, crocheteur; empocheter, poche, pochetée; faner, fener; foin; gérémium, bec-de-grue; mulon, veuilloche; orge mondaine; mauve des jardins, passe-rose, rose-d'outremer, rose-tremière; pelleter; pesâ; racérer.

\section{L'être humain et la vie sociale}

\section{Les relations amoureuses et sexuelles}

amant, amante, faire l'amour, blonde, aller voir sa blonde, fort sur la blonde, amoureux de 36 blondes, cavalier; créature; aller voir, fréquenter la créature, ébraillée (subst.), fille publique; faro, farôder, fignoler, fignoleur; courir la galipote; marier qqn.

\section{Le langage et les rapports de société}

avoir affaire de; balbutier; brasser, (se) brouscailler; se déboutonner; décent; tomber comme le ginseng; baragouin, micmac, ratapiat; être ordonné de; papier-nouvelle, papier-public; payer ses complimens (ses respects, une visite).

\section{Les actions violentes exercées contre qqn}

batailleur; bourgogner; donner, fricasser une dégelée, donner, fricasser une ramasse; ébrayer; écrapoutiller, écrapoutir, écrapoutir comme un crapaud; en donner sur les épicailles; fesser; graisse, graisser; mècher, moucher; tapin; verbalement.

\section{Les jeux et les distractions}

balançoire, berlancille, brandilloire, escarpolette, se balancer, berlanciller, se brandiller; bombarde, guimbarde, trompe; canot, canoter, canoteur; battoir, raquette, pelote, traîneau.

\section{Les métiers}

agriculteur, habitant, habitante, laboureur, paysan; appointement, appointer; cabaret, cabaretier, cantine, cantinier, détailleur, taverne, tavernier; canoter, canoteur; manchonnier; niveleux; voyageur. 


\section{Le commerce}

avoir plus d'acquet, avoir plus de quitte; argent; coppre; courir les côtes; étau; faire ses georges; houiller; office; retraiter, retrayer.

\section{Le nombre et la quantité}

une beauté, deusse, fièrement, troisse; canot, canotée, charretée, jument, poche, pochetée, voie, voyage.

\section{La propriété}

coureur de côtes, courir les côtes; fripper, glumer, en licher à qqn; gueusasse, gueusaille; quêter, quêteur.

\section{La vie militaire}

belt; corporal; faire coup; couronel; factionnaire, sentinelle; matelat; retraiter.

\section{La maison, les meubles}

amancher, démancher, ramancher; apichimon; bagage, butin, drigaille, ménage; ber; buffet; cadre; couette; escabeau; premier étage; lévier; tapisserie.

\section{Les ustensiles et les récipients}

bombe, bouilloire, canard; casseau, meuble «vase, plat»; ébaroui, s'ébarouir; lichefrite; micoine; plated; sieau; thétière.

\section{Le chauffage}

attisée; boucane, boucaner; écopeau; sec comme un écopeau; flambe; tondre «amadou»; tuer «éteindre».

\section{Les travaux domestiques}

amidon, empois; balier; brasser le poêle, brasser le chaudron; bredas, bredasser; fer à flasquer; rapiester; train «ménage».

\section{Le transport}

\section{Par voie de terre}

amont la côte; berline, bordel, cabrouet, calèche, cariole; boyard, brancard, civière; brancard, menoirs, travail; cahot; cannevette, cantine; claque, mèche; cordeaux, courroies, guides, rênes; débarquer, embarquer; plancher des vaches; robe de bœuf; traîne, traîne de clisse.

\section{Par voie d'eau}

cage, cajeux, crible, dram; canot, canoter. 


\section{Onomastique}

avoir, être une tête d'Allemand; malin comme un Basque; Canadien; malin comme un Cris ; galeux comme un Écossois ; Juifrèsse ; Montréaliste ; Québéquois, Québecquois; Sauvage, Sauvagesse; Sorel. 


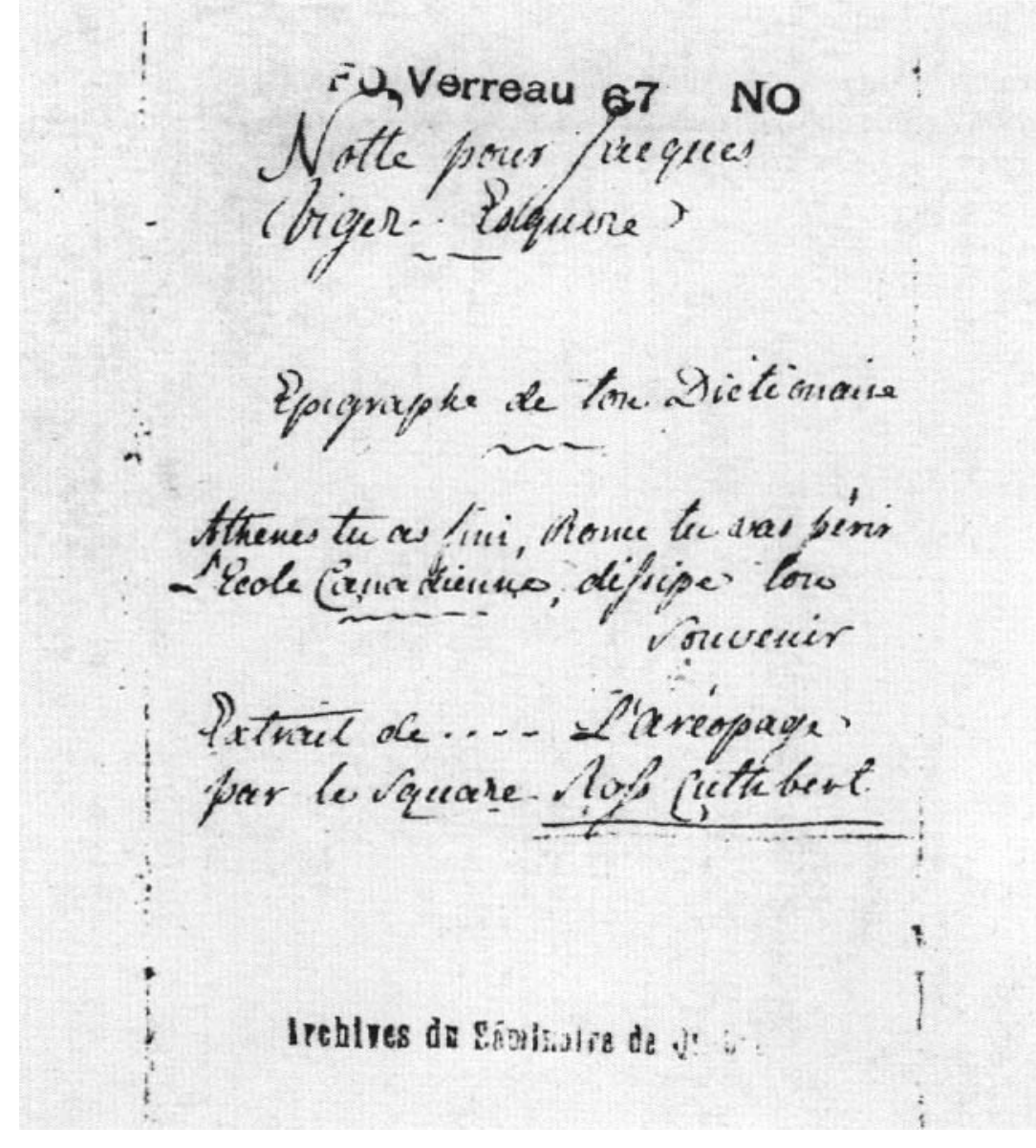

Épigraphe adressée à Jacques Viger par Ross Cuthbert, tirée de son ouvrage L'Aréopage, publié en 1803. Manuscrit conservé au Musée de la civilisation, fonds d'archives du Séminaire de Québec. 


\section{LES COMPARAISONS}

Les nombreuses comparaisons que Viger a consignées, ainsi que celles qui figurent dans les Appendices, nous ont incitée à en faire le relevé. Comme les comparaisons figurant dans les Appendices datent, comme le texte de Viger, du début du XIX ${ }^{\mathrm{e}}$ siècle, la fusion des deux sources allait de soi. La méthodologie que nous avons adoptée est celle qu'a utilisée $R$. Lepelley dans son étude sur les comparaisons en Normandie, intitulée : "Les comparaisons dans le Val de Saire (Basse-Normandie)" (v, bibliogr.).

L'auteur s'est lui-même inspiré d'un article de J. Cohen sur le sujet : "La comparaison poétique : essai de systématique» (v. bibliogr.). La terminologie qu'il a adoptée est celle qu'a utilisée J. Cohen dans son article, soit : $\mathrm{A}$ est $\mathrm{B}$ comme $\mathrm{C}$, où $\mathrm{B}$ est le prédicat ou sème commun aux deux lexèmes dont l'un, $A$, est le "comparé» et l'autre, $\mathrm{C}$, le «comparant» (Cohen 44).

Dans son article, $R$. Lepelley pose la question de la pertinence de l'étude des comparaisons en ces termes : "N'est-il pas possible de mieux connaittre les habitants d'une région, leurs activités et leurs mentalités, en étudiant les comparaisons dont ils émaillent leur discours?» En effet, l'étude des comparaisons nous apprend beaucoup sur l'état d'esprit de ceux qui les utilisent. Comme le démontre l'auteur dans son étude, les comparaisons "correspondent à une certaine manière d'être et à une certaine manière de penser»; d'où l'intérêt d'en tenir compte dans les études sur la langue.

\section{PRÉDICATS}

aimer

amoureux

blême

boire

brailler

chacun à son tour

chanter

\section{LOCUTIONS}

j'aime cela comme la colique

il est amoureux comme une chatte

elle est blême comme un linge

il boit comme un trou

il braille comme un veau

chacun à son tour comme au moulin

il chante comme un perdu

il chante comme un rêve 
connaître

connu

couler

courir

crier

curieux

dormir

droit

dur

écrapoutiller

être

fier

fort

fou

frète

galeux

gras

grossier

haut

jambes

jouqué

laid

leste

lever le cul

long

malade je connois cela comme ma poche

il est connu comme Barrabbas à la Passion

ça coule comme un sas

il court comme un chat sauvage

crier comme un sourd

curieux comme la chèvre à Jacques Cartier

il dort comme une soupe

droit comme un piquet

il est dur comme un cheval

écrapoutiller comme un crapaud

c'est comme le moulin de La Chine, qui ne s'arrête plus quand il est parti

il est comme l'oiseau sur la branche

c'est comme une mitaine sans pouce

c'est comme une troisième roue à une charrette

c'est comme un pou sur une gale

fier comme un Écossois

il est fort comme un Turc

fou comme braque

frète comme glace

galeux comme un Écossois

gras comme un voleur

il est grossier comme un pain d'orge

haut comme un chou

il a les jambes comme des bâtons de traîne

il est jouqué comme un dinde

laid comme un grichou

leste comme un chien de plomb

il lève le cul comme une bête puante

c'est long comme d'icite à demain

malade comme un chien 
malin

manger

marcher

mentir

parler

partir

passer net

pauvre

prendre au nez

prendre du café

propre

reçu

renflé

savant

sec

sot

tête

tomber malin comme un Basque

malin comme un Cris

il est malin comme sept fois le diable

il est malin comme un grichou

il mange comme un goulia

il marche comme un chien qui vient des vêpres

il ment comme un arracheur de dents

il parle comme saint Paul

il parle comme la Bouche

parler comme une femme

tu pars comme un fusil sans plaque

le mal passe net comme sur la main

pauvre comme un rat d'église

cela prend au nez comme la moutarde

il prend du café comme la Brillant

propre comme un sou

il a été reçu comme un chien à vêpres

il a été reçu comme un chien dans un jeu de quilles

renflé comme une soupe au lait

savant comme un livre

sec comme un coton de bled d'Inde

sec comme un écopeau

sec comme un manche de balai

sec comme un pain d'épice

sec comme nord-est

sec comme un pendu

sot comme un fondeur de cloches

il a la tête comme un procès-verbal

il est tombé comme le ginseng

ça tombe comme la pauvreté sur le monde 
tomber comme une tourte

trempe

il est revenu trempe comme une soupe

tuer roide

tuer roide comme une tourte

unis

les chemins unis comme des chemins de cartes

vide corps vide comme un fanal

\section{Liste alphabétique des «comparants»}

\begin{tabular}{ll} 
"COMPARANTS» & PRÉDICATS \\
arracheur de dents & mentir \\
Barrabbas à la Passion & connu \\
Basque & malin \\
bâtons de traine & jambes \\
bête puante & lever le cul \\
Bouche & parler \\
braque & fou \\
Brillant & prendre du café \\
chat sauvage & courir \\
chatte & amoureux \\
chemins de cartes & unis \\
cheval & dur \\
chèvre à Jacques Cartier & curieux \\
chien & malade \\
chien à vêpres & reçu \\
chien dans un jeu de & reçu \\
quilles & \\
chien de plomb & leste \\
chien qui vient des & marcher \\
vêpres & \\
chou & haut \\
colique & aimer \\
coton de bled d'Inde & sec \\
\hline
\end{tabular}




\begin{tabular}{ll} 
crapaud & écrapoutiller \\
Cris & malin \\
dinde & jouqué \\
écopeau & sec \\
Écossois & fier \\
Écossois & galeux \\
fanal & vide \\
femme & parler \\
fondeur de cloches & sot \\
fusil sans plaque & partir \\
ginseng & tomber \\
glace & frète \\
goulia & manger \\
grichou & laid \\
grichou & malin \\
icite à demain (d' ) & long \\
linge & blême \\
livre & savant \\
main (sur la ) & passer net \\
manche de balai & sec \\
mitaine sans pouce & être \\
moulin & chacun à son tour \\
moulin de La Chine & être \\
moutarde & prendre au nez \\
nord-est & sec \\
oiseau sur la branche & être \\
pain d'épice & sec \\
pain d'orge & grossier \\
pauvreté sur le monde & tomber \\
pendu & sec \\
perdu & chanter \\
\hline
\end{tabular}




$\begin{array}{ll}\text { piquet } & \text { droit } \\ \text { poche } & \text { connaître } \\ \text { pou sur une gale } & \text { être } \\ \text { procès-verbal } & \text { tête } \\ \text { rat d'église } & \text { pauvre } \\ \text { rêve } & \text { chanter } \\ \text { saint Paul } & \text { parler } \\ \text { sas } & \text { couler } \\ \text { sept fois le diable } & \text { malin } \\ \text { sou } & \text { propre } \\ \text { soupe } & \text { dormir } \\ \text { soupe } & \text { trempe } \\ \text { soupe au lait } & \text { renflé } \\ \text { sourd } & \text { crier } \\ \text { tourte } & \text { tomber } \\ \text { tourte } & \text { tuer roide } \\ \text { troisième roue à une } & \text { être } \\ \text { charrette } & \\ \text { trou } & \text { boire } \\ \text { Turc } & \text { fort } \\ \text { veau } & \text { brailler } \\ \text { voleur } & \text { gras } \\ \end{array}$




\section{BIBLIOGRAPHIE}

\section{MANUSCRITS}

GrondIN, François-Xavier, «Bio-bibliographie de Jacques Viger», thèse dactylographiée, École des bibliothécaires, Université de Montréal, 1947, 2 vol., $359 \mathrm{p}$.

VIGER, Jacques, «Néologie Canadienne, ou Dictionnaire des mots créés en Canada \& maintenant en vogue; - des mots dont la prononciation \& l'ortographe sont différentes de la prononciation \& ortographe françoises, quoique employés dans une acception semblable ou contraire; et des mots étrangers qui se sont glissés dans notre langue», 1810 ; manuscrit classé au fonds Verreau, $\mathrm{n}^{\circ} 67$, liasses I B et 2, au Musée de la civilisation, fonds d'archives du Séminaire de Québec. (Les notes et listes de mots qui figurent dans les Appendices se trouvent également sous cette cote.)

VIGER, Jacques, «La Saberdache» qui comprend : «La Saberdache rouge» $\mathrm{n}^{\infty} 0-95$ à 0-125 et «La Saberdache bleue» $n^{\infty} 0-139$ à 0-152; manuscrits conservés au fonds Verreau, au Musée de la civilisation, fonds d'archives du Séminaire de Québec.

\section{IMPRIMÉS}

Académie = Le Dictionnaire de l'Académie franfoise, Paris, Jean-Baptiste Coignard, 1694, 2 vol.; $2^{\mathrm{e}}$ éd., Nouveau dictionnaire de l'Académie frangoise, Paris, J.-B. Coignard, 1718, 2 vol.; $3^{\mathrm{e} e ́ d ., ~ D i c t i o n n a i r e ~ d e ~ l ' A c a d e ́ m i e ~ f r a n f o i s e, ~ P a r i s, ~ J .-B . ~}$ Coignard, 1740, 2 vol.; $4^{e}$ éd., Dictionnaire de l'Académie franfoise, Veuve de Bernard Brunet, 1762, 2 vol.; $5^{\circ}$ éd., Dictionnaire de l'Académie franfoise, Paris, J.J. Smits, 1798, 2 vol.; $6^{\mathrm{e}}$ éd., Dictionnaire de l'Académie françoise, Paris, Firmin Didot Frères, 1835, 2 vol.; $7^{e}$ éd., Dictionnaire de l'Académie française, Paris, Firmin Didot, 1878, 2 vol.; $8^{\mathrm{C}}$ éd., Dictionnaire de l'Académie française, Paris, Hachette, 1932-1935, 2 vol.

AcCompl = Complément du Dictionnaire de l'Académie franfaise, Paris, Firmin Didot Frères, 1844, XXXII-1281 p.

Arveiller = ARVEILIER, Raymond, Contribution à l'etude des termes de voyage en franfais (1505-1722), Paris, Éditions d'Artrey, 1963, 571 p.

AUdisio, Gabriel et Isabelle BONNOT-RAMBAUD, Lire le franfais d'bier: manuel de paléographie moderne $X V^{e}-X V I I I^{e}$ siècle, Paris, Armand Colin, 1991, $252 \mathrm{p}$.

Aurore ( $\left.L^{\prime} \sim\right)$, hebd., fondateurs Michel Bibaud et Joseph-Victor Delorme, Montréal, 10 mars 1817 - sept. 1819.

Baraga $=$ BARAGA, Frederic, $A$ Dictionary of the Otchipwe Language, explained in English : part I, English-Otchipwe by R. R. Bishop Baraga, Montréal, Beauchemin \& Valois, 1878, $301 \mathrm{p}$. 
Barre du jour ( $L a \sim$ ), revue littéraire bimestrielle, Bois-des-Filions (Québec), La Barre du jour, 1965-1977, 57 vol. Continuée par La Nouvelle barre du jour depuis 1977.

BARTHE, Joseph-Guillaume, Souvenirs d'un demi-siècle ou Mémoires pour servir à l'bistoire contemporaine, Montréal, J. Chapleau \& fils, 1885, XVII-482 p.

Bauche = BAUCHE, Henri, Le langage populaire : grammaire, syntaxe et dictionnaire du français tel qu'on le parle dans le peuple de Paris avec tous les termes d'argot usuel, nouv. éd., Paris, Payot, 1946, 231 p.

Bélisle = BÉLISLE, Louis-Alexandre, Dictionnaire nord-américain de la langue française, éd. entièrement refondue comprenant : suppléments de biographie, histoire, géographie, et des plus importantes villes du monde avec leur population, Montréal, Beauchemin, 1979, [XIV]-1196 p.

Bescherelle $=$ BESCHERELLE, Louis-Nicolas, Dictionnaire national ou Dictionnaire universel de la langue française, $2^{\mathrm{e}}$ éd., Paris, Garnier, 1845-1846, 2 vol.; $6^{\mathrm{e}}$ éd., Paris, Garnier, 1858, 2 vol.

Bibaud, Maximilien, Le pantbéon canadien : choix de biograpbies, Montréal, J. M. Valois, 1891, VI-320 p.

Bibliothèque canadienne, ou miscellanées historiques, scientifiques, et littéraires ( $\mathrm{La} \sim)$, revue mensuelle, fondateur-propriétaire et rédacteur Michel Bibaud, Montréal, juin 1825 - 5 juin 1830.

Blais Top = BLAIS, Suzelle, Apport de la toponymie ancienne aux études sur le franfais québécois et nord-américain : documents cartograpbiques du Régime français, Québec, Commission de toponymie, 1983, IX-105 p. Études et recherches toponymiques, 6. Cartes.

Boiste = Boiste, Pierre-Claude-Victoire, Dictionnaire universel de la langue franf̧aise, avec le latin et les étymologies, $7^{\mathrm{e}}$ éd., Paris, Verdière libraire, 1829, XX-724 p. ; compl. 210 p. ; 8 éd. rev., corr. et considérablement augm. par Charles Nodier, Paris, Lecointe et Pougin libraires, 1834, XXIV-756 p.; compl. $241 \mathrm{p}$.

BouchHist = BOUCHER, Pierre, Histoire véritable et naturelle des mours E productions du pays de la Nouvelle France, vulgairement dite le Canada, Paris, Florentin Lambert, 1664, 168 p.; réimpr. : la Société historique de Boucherville, [Boucherville], 1964, LXIII- 415 p. (Le texte de Boucher se trouve aux pages 1 à 168.)

BouHours, Dominique, Remarques nouvelles sur la langue franfoise, Paris, Sebastien Mabre-Cramoisy, 1675, XX-540 p.; Suite des Remarques nouvelles sur la langue françoise, Paris, George \& Loüis Josse, 1687, XXII-469 p.; réimpr. : Slatkine Reprints, Genève, 1973.

Bourciez = BourcieZ, Édouard et Jean BourcieZ, Phonétique française, Paris, Éditions Klincksieck, 1974, XII-243 p.

Boyer $=$ BOYER, Abel, Dictionnaire royal franfois-anglois et anglois-franfois; tiré des meilleurs auteurs, gui ont écrit dans ces deux langues. Soigneusement rev., corr. $\mathcal{G}^{\circ}$ augm. d'un très grand nombre de mots $\mathcal{E}^{\circ}$ de pbrases, de plusieurs idiotismes, et de différentes constructions $\mathcal{E}^{2}$ façons de parler, tant françoises quangloises, Londres, C. Bathurst, 1773, (s. p.).

$\mathrm{BPFC}=$ Bulletin du parler français au Canada, Québec, La Société du parler français au Canada, vol. 1-16, 1902-1918. 
$\mathrm{BRH}=$ Bulletin des recherches historiques, Lévis-Québec, Pierre-Georges Roy puis Antoine Roy à partir de 1949, 1895-1968.

$\mathrm{BW}=\mathrm{BLOCH}$, Oscar et Walther von WARTBURG, Dictionnaire étymologique de la langue franfaise, $5^{\mathrm{e}}$ éd., Paris, Presses Universitaires de France, 1968, XXXVI$682 \mathrm{p}$.

Canadiana Romanica, Franfais du Canada - Français de France, Actes du deuxième Colloque international de Cognac du 27 au 30 septembre 1988, publiés par Brigitte Horiot, Tübingen, Max Niemeyer Verlag, vol. 6, 1991, 236 p.

Canadiana Romanica, Franfais du Canada - Français de France, Actes du troisième Colloque international d'Augsbourg du 13 au 17 mai 1991, publiés par HansJosef Niederehe et Lothar Wolf, Tübingen, Max Niemeyer Verlag, vol. 7, $1993,256 \mathrm{p}$.

Canadien (Le $\sim$ ), hebd., fondateurs Pierre Bédard et François-Xavier Blanchet, Québec, 22 nov. 1806 - 14 mars $1810 ; 14$ juin 1817 - 15 déc. $1819 ; 19$ janv. 1820 -2 mars 1825; 7 mai 1831 - 11 févr. 1893.

CanBAloi = Canadianismes de bon aloi, Cahiers de l'Office de la langue française, $\mathrm{n}^{\circ}$ 4, Québec, ministère des Affaires culturelles, 1969, 37 p.; éd. rev. et corr., Québec, ministère de l'Éducation, 1973, 11 p.

Caradec = CARADEC, François, N'ayons pas peur des mots $:$ dictionnaire du français argotique et populaire, Paris, Larousse, 1988,319 p.

CartNord = CARTON, Fernand et Denise POULET, Dictionnaire du français régional du Nord-Pas-de-Calais, Paris, Éditions Bonneton, 1991, 125 p.

CAYrou, Gaston, Le français classique : lexique de la langue du dixx-septième siècle expliquant d'après les dictionnaires du temps et les remarques des grammairiens le sens et l'usage des mots aujourd'bui vieillis ou différemment employés, Paris, Didier, 1948, $884 \mathrm{p}$.

Champlain, Samuel de, Euvres de Cbamplain (1598-1632), publiées sous le patronage de l'Université Laval, par C.-H. Laverdière, $2^{2}$ éd., Québec, G.-E. Desbarats, 1870; réimpr. : Georges-Émile Giguère, Montréal, Éditions du Jour, 1973, 6 t. en 3 vol. Cartes. Illustrations.

ChaudÉtude $=$ CHAunEnSON, Robert, «Pour une étude comparée des créoles et parlers français d'outre-mer : survivance et innovation», dans $R L i R$, t. 37 , $n^{\propto 1} 147-148$, juillet-déc. 1973 , p. 342-371.

ChaudRéun = CHAUdenson, Robert, Le lexique du parler créole de la Réunion, Paris, Librairie Honoré Champion, 1974, 2 vol., XLIX-1249 p.

ClapAmer = CLAPIN, Sylva, $A$ New Dictionary of Americanisms Being a Glossary of Words Supposed to Be Peculiar to the United States and the Dominion of Canada, New York, Louis Weiss \& Co., 1902; réimpr. : Gale Research Company, Detroit, 1968, XV-581 p.

Clapin = CLAPIN, Sylva, Dictionnaire canadien-français ou Lexique-glossaire des mots, expressions et locutions ne se trouvant pas dans les dictionnaires courants et dont l'usage appartient surtout aux Canadiens-français, Montréal, C.O. Beauchemin \& Fils, et Boston, Sylva Clapin, 1894; réimpr. : Les Presses de l'Université Laval, Québec, 1974, XIVI-389 p.

COHEN, Jean, «La comparaison poétique : essai de systématique», dans Langages, $n^{0} 12,1968$, p. 43-51.

ColPron, Gilles, Les anglicismes au Québec, Montréal, Librairie Beauchemin limitée, 1975,247 p. 
Corneille = CORNEILLE, Thomas, Le dictionnaire des arts et des sciences, Paris, JeanBaptiste Coignard, 1694, 2 vol.; réimpr. : Slatkine Reprints, Genève, 1968.

CorrVoit = CORRIVEAU, Claude, Les voitures à chevaux au Québec, Sillery (Québec), Les Éditions du Septentrion, 1991, 172 p.

Cotgrave $=$ COTGRAVE, Randle, $A$ Dictionarie of the French and English Tongues, London, Adam Islip, 1611, (s.p.); réimpr. :The Scolar Press Limited, Menston, England, 1968.

CuoqAlg = Cuod Jean-André, Lexique de la langue algonquine, Montréal, J. Chapleau, 1886,446 p.

Cuthbert, Ross, L'Aréopage, Québec, printed by John Neilson, 1803, 13 p.

Cyr, Céline, «Michel Bibaud», dans $D B C$, vol. VIII, de 1851 à 1860, 1985, p. $97-$ 99.

Dagenais = DAGENAIS, Gérard, Dictionnaire des difficultés de la langue française au Canada, 2 éd., Boucherville, Les Éditions françaises inc., 1984, 538 p.

DANET, Pierre, Grand dictionnaire franfois et latin; enrichi des meilleures façons de parler en l'une et l'autre langue; avec des notes de critique et de grammaire, nouv. éd., rev., corr. et augm. considérablement par l'auteur, Lyon, Deville Frères \& L. Chalmette, $1735,1256 \mathrm{p}$.

DarbDict = DARBELNET, Jean, Dictionnaire des particularités de l'usage, éd. rev. et corr., Sillery (Québec), Presses de l'Université du Québec, 1988, 215 p.

DavLang = DAVIAULT, Pierre, Langage et traduction, Ottawa, Bureau fédéral de la traduction, Secrétariat d'État, 1963, 397 p.

DavTour = DAVAU, Maurice, Le vieux parler tourangeau : sa phonétique, ses mots et locutions, sa grammaire, Chambray-lès-'Tours, C.L.D., 1979, 505 p.

$\mathrm{DBC}=$ Dictionnaire biographique du Canada / Dictionary of Canadian Biography, Québec, Les Presses de l'Université Laval, et Toronto, University of Toronto Press, en cours de publication depuis 1966.

Delvau $=$ DELVAU, Alfred, Dictionnaire de la langue verte : argots parisiens comparés, Paris, E. Dentu, 1866, XVI-406 p.

DepMots = DEPECKER, Loïc, Les mots des régions de France, collection «Le français retrouvé», Paris, Éditions Belin, 1992, 447 p.

$\mathrm{DFC}=$ DuBOIS, Jean et autres, Dictionnaire du franfais contemporain, Paris, Larousse, 1980, XXXII-1263 p.

$\mathrm{DFCl}=$ DuBOIS, Jean, René LAGANE et Alain LEROND, Dictionnaire du français classique, le XVII siècle, Paris, Larousse, 1992, XXIX-511.

$\mathrm{DFP}=$ Dictionnaire du franfais plus à l'usage des francophones d'Amérique, sous la responsabilité de A. E. Shiaty, Montréal, Centre éducatif et culturel inc., 1988, XXIV-1856 p.

$\mathrm{DFQ}=$ Dictionnaire du franfais québécois; description et bistoire des régionalismes en usage au Québec depuis l'époque de la Nouvelle-France jusqu'à nos jours incluant un apergu de leur extension dans les provinces canadiennes limitrophes, volume de présentation, sous la direction de Claude Poirier, Sainte-Foy, Les Presses de l'Université Laval, 1985, XXXIII-167 p.

DG = HATZFELD, Adolphe, Arsène DARMEsTeTER et Antoine THOMAS, Dictionnaire général de la langue française du commencement du XVIT siècle jusquà nos jours, $6^{\mathrm{e}}$ éd., Paris, Delagrave, 1920, 2 vol. 
DictCan $=A$ Dictionary of Canadianisms on Historical Principles, Editor-in-Chief Walter S. Avis, Toronto, W. J. Gage Limited, 1967, XXIII-927 p.

Dictionnaire des parlementaires du Québec, 1792-1992, sous la direction de Gaston Deschênes, Sainte-Foy, Les Presses de l'Université Laval, 1993, 859 p.

Dionne = DiONNE, Narcisse-Eutrope, Le parler populaire des Canadiens français ou Lexique des canadianismes, acadianismes, anglicismes, américanismes, mots anglais les plus en usage au sein des familles canadiennes et acadiennes françaises, Québec, Laflamme \& Proulx imprimeurs, 1909, XXIV-671 p.; réimpr. : Les Presses de l'Université Laval, Québec, 1974.

DitchyLouis = DiTCHY, Jay Karl, Les Acadiens louisianais et leur parler, Paris, Librairie E. Droz, 1932, 272 p.

DorrSteGen = DORRANCE, Ward Allison, «The Survival of French in the Old District of Sainte Genevieve», dans The University of Missouri Studies, Columbia (Missouri), vol. 10, $\mathrm{n}^{\circ} 2$, avril 1935, $133 \mathrm{p}$.

DQA = Dictionnaire québécois d'aujourd'bui ; langue française, bistoire, géograpbie, culture générale, rédaction dirigée par Jean-Claude Boulanger, supervisée par Alain Rey, Saint-Laurent (Québec), DicoRobert Inc., 1992, XXXV-1269 p.; noms propres 343 p.; annexes LXII.

DubGloss = DuboIs, Ulysse et autres, Glossaire des parlers populaires de Poitou, Aunis, Saintonge, Angoumois, nouv. éd., Les Granges, Société d'études folkloriques du Centre-Ouest, 1992, $252 \mathrm{p}$.

DugGent = Dugas, Jean-Yves, Répertoire des gentilés du Québec, Québec, Les Publications du Québec, 1987, 258 p. Études et recherches toponymiques 12.

DulDictC = Dulong, Gaston, Dictionnaire des canadianismes, Larousse Canada, $1989,461 \mathrm{p}$.

DumPron = DuMAS, Denis, Nos façons de parler : les prononciations en français québécois, Sillery (Québec), Presses de l'Université du Québec, 1987, XIV-155 p.

DunBouq = DuNETON, Claude, en coll. avec Sylvie CLAVAL, Le bouquet des expressions imagées : encyclopédie thématique des locutions figurées de la langue franfaise, Paris, Éditions du Seuil, 1990, 1375 p.

Dunn = DUNN, Oscar, Glossaire franco-canadien et vocabulaire de locutions vicieuses usitées au Canada, Québec, Imprimerie A. Côté et Cie, 1880, XXVI-199 p.; réimpr. : Québec, Les Presses de l'Université Laval, 1976.

Dupré = DUPRÉ, P., Encyclopédie du bon franfais dans l'usage contemporain, Paris, Éditions deTrévise, 1972, 3 vol.

ÉDOUARD, Robert, Dictionnaire des injures, Paris, Éditions Tchou, 1979, 334 p.

Encyclopédie = Encyclopédie ou Dictionnaire raisonné des sciences, des arts et des métiers, mis en ordre et publ. par MM. Diderot et D'Alembert, t. 1-7, Paris, Briasson - David l'aîné - Le Breton - Durand, 1751-1757; t. 8-17, Neufchastel, Samuel \& Compagnie libraires \& imprimeurs, 1765.

Encyclopédie Canadienne ; journal littéraire et scientifique $\left(L^{\prime} \sim\right)$, mensuel, fondateur, propriétaire et rédacteur Michel Bibaud, Montréal, imprimeur J. Lovell, mars 1842 - févr. 1843.

Études de linguistique franco-canadienne: communications présentées au XXXIV Congrès de l'Association canadienne-française pour l'avancement des sciences (Québec, novembre 1966), publiées par Jean-Denis Gendron et Georges Straka, Paris, Klincksieck et Québec, Les Presses de l'Université Laval, 1967, 176 p. 
Études sur le parler français au Canada, Québec, Les Presses universitaires Laval, $1955,220 \mathrm{p}$.

Fabvre = FABVRE, Bonaventure, Racines montagnaises compilées à Tadoussac avant 1695 par le père Bonaventure Fabvre, jésuite, pré-transcription Lorenzo Angers, transcription définitive et édition de Gerard E. McNulty, Québec, Université Laval, 1970, 387 p.

Féraud = FÉRAUD, Jean-François, Dictionnaire critique de la langue française, Marseille, Jean Mossy, 1787-1788, 3 vol.; réimpr. : Max Niemeyer Verlag, Tübingen, 1994.

FERTIAULT, François, Dictionnaire du langage populaire verduno-chalonnais (Saôneet-Loire), Paris, Bouillon, 1890; réimpr. : Laffitte Reprints, Marseille, 1980, $472 \mathrm{p}$.

FEW = WARTBURG, Walther von, Französisches Etymologisches Wörterbuch. Eine Darstellung des galloromanischen Spracbschatzes, Bonn puis Bâle, en cours de publication depuis 1922, 152 fascicules parus.

Furetière = FURETIÈRE, Antoine, Dictionnaire universel, contenant generalement tous les mots françois tant vieux que modernes, $\mathcal{G}$ les termes de toutes les sciences et des arts, La Haye-Rotterdam, Arnout \& Reinier Leers, 1690, 3 vol.; réimpr. : Dictionnaire Le Robert, Paris, 1978, 3 vol.; Dictionnaire universel, La Haye, Pierre Husson et autres, 1727; réimpr. : Georg Olms Verlag, Hildesheim New York, 1972, 4 vol.

Gage = Gage Canadian Dictionary, Toronto, Gage Educational Publishing Company, 1983, XXX-1313 p.

Gagnon = GAGNON, Philéas, «La langue parlée au Nord-Ouest canadien», dans $B P F C$, vol. VI, 1907-1908, p. 136.

Gardette, Pierre, «Pour un dictionnaire de la langue canadienne», dans $R L i R$, t. XVIII, n ${ }^{\infty 6} 69-70$, janv.-juin 1954 , p. 85-100.

GAUTHIER, Henri, La compagnie de Saint-Sulpice au Canada, Montréal, Séminaire de Saint-Sulpice, 1912, $150 \mathrm{p}$.

GendrPhon = GENDRON, Jean-Denis, «Le phonétisme du français canadien du Québec face à l'adstrat anglo-américain", dans Études de linguistique francocanadienne, 1967, p. 15-63.

GendrRur = GENDRON, Jean-Denis, «Contribution à l'étude du français rural parlé au Canada", dans TraLiLi, t. IV, 1, 1966, p. 173-189.

GendrTend = GENDRON, Jean-Denis, Tendances phonétiques du français parlé au Canada, Paris, Librairie Klincksieck, et Québec, Les Presses de l'Université Laval, 1966, XX-254 p.

GHarrap = Grand Harrap : dictionnaire français-anglais et anglais-français, éd. rev. par R. P. L. Ledésert et Margaret Ledésert, London, Harrap, 1981, 4 vol.

GLar $=$ Grand Larousse en 5 volumes, Paris, Librairie Larousse, 1987, 5 vol.

GLLF = Grand Larousse de la langue francaise, sous la direction de Louis Guilbert, René Lagane et Georges Niobey, Paris, Librairie Larousse, 1971-1978, 7 vol.

Glossaire = Glossaire du parler français au Canada, préparé par la Société du parler français au Canada, Québec, L'Action sociale limitée, 1930, XIX-709 p.; réimpr. : Les Presses de l'Université Laval, Québec, 1968. 
GodCompl = GODEFROY, Frédéric, Dictionnaire de l'ancienne langue française et de tous ses dialectes du $I X^{e}$ au $X V^{e}$ siècle, Paris, F. Vieweg, 1893-1902, vol. 8-10; réimpr. : Slatkine, Genève-Paris, 1982.

Godefroy = GODEFroY, Frédéric, Dictionnaire de l'ancienne langue française et de tous ses dialectes du $I X^{e}$ au $X V^{e}$ siècle, Paris, F. Vieweg, 1891-1902, 10 vol.; réimpr. : Slatkine, Genève-Paris, 1982.

GodOis = Godfrey, W.-Earl, Les oiseaux du Canada, Ottawa, Musée national du Canada, bulletin $n^{\circ} 203,1976,506$ p.

GOUGENHEIM, Georges, Les mots franfais dans l'bistoire et dans la vie, Paris, A. \& J. Picard \& Cie, 1962-1975, 3 vol.

GougÉtude = GOUGENHEIM, Georges, Étude sur les périphrases verbales de la langue franfaise, Paris, Librairie A.-G. Nizet, 1971, 383 p.

Grevisse = GREvisse, Maurice, Le bon usage : grammaire franfaise avec des remarques sur la langue franfaise d'aujourd'bui, $8^{\mathrm{e}}$ éd. rev., Gembloux (Belgique), Editions J. Duculot et Paris, Librairie A. Hatier, 1964, 1194 p.

GRob = ROBERT, Paul, Le Grand Robert de la langue française. Dictionnaire alphabétique et analogique de la langue française, $2^{\mathrm{e}}$ éd., entièrement revue et enrichie par Alain Rey, Paris, Le Robert, 1992, 9 vol.

Grondin, François-Xavier, Jacques Viger, Montréal, 1942, 31 p.

GuirÉtym = GurRaud, Pierre, Dictionnaire des étymologies obscures, Paris, Payot, $1982,523 \mathrm{p}$.

HaignBoul = HAIGNERÉ, Daniel, Le patois boulonnais comparé avec les patois $d u$ Nord de la France, Boulogne-sur-Mer, Deligny, 1903, 638 p.

HALlig, Rudolf et Walther von WARTBURG, Système raisonné des concepts pour servir de base à la lexicograpbie : essai d'un schéma de classement, $2^{e}$ éd. recomp. et augm., Berlin, Akademie Verlag, 1963, 315 p.

HalPot = HALFORD, Peter W., Le français des Canadiens à la veille de la Conquête : témoignage du père Pierre Pbilippe Potier, s.j., collection «Amérique française», $\mathrm{n}^{\circ} 2$, Ottawa, Les Presses de l'Université d'Ottawa, 1994, XI-380 p.

HAMELIN, Jean, «La dimension historique du problème linguistique», dans TraLiQ, vol. 3, Québec, Les Presses de l'Université Laval, 1979, p. 251-269.

HAMELIN, Louis-Edmond, Le rang d'babitat : le réel et l'imaginaire, Cahiers du Québec, collection «Géographie», LaSalle (Québec), Editions Hurtubise HMH Ltée, 1993, 328 p.

Hanse = HANSE, Joseph, Nouveau dictionnaire des difficultés du français moderne, Paris-Gembloux, Éditions Duculot, 1983, 1014 p.

HARrIS, Richard Colebrook, The Seigneurial System in Early Canada : $A$ Geographical Study, Madison, University of Wisconsin Press, 1966; reprinted with a new preface, Montréal and Kingston, McGill-Queen's University Press, 1984, XXIV-247 p.

HENRY, Alexander, Travels and Adventures in Canada and the Indian Territories, between the years 1760 and 1776, New York, I. Riley, 1809, 330 p.; réimpr. : University Microfilms, Inc., Ann Arbor (Michigan), 1966.

House $=$ HoUSE, A. B. et Noël L. CORBETT, «Sur l'origine de la prononciation icit «ici» au Canada français», dans Le Français moderne, $n^{\circ} 38,1970$, p. 147150. 
Huguet = HUGUET, Edmond, Dictionnaire de la langue franfaise du seizième siècle, Paris, Didier, 1925-1967, 7 vol.

HumbGen = HUMBERT, Jean, Nouveau glossaire genevois, Genève, Jullien, 1852, 2 vol.; réimpr. : Slatkine, Genève, 1970, 2 t. en 1 vol.

Huston, James, Le Répertoire national ou Recueil de littérature canadienne, Montréal, Lovell et Gibson, 1848, 4 vol.

JaubCentre = JAUBERT, Hippolyte-François de, Glossaire du Centre de la France, suivi d'un supplément, Paris, Chaix, 1864, 732 p.

Journet $=$ Journet, René, Jacques Petir et Guy ROBERT, Mots et dictionnaires (1798-1878), Annales littéraires de l'Université de Besançon, Paris, Belles Lettres, 1966-1978, 11 vol.

JournJés = Journal des jésuites, publié d'après le manuscrit original conservé aux archives du Séminaire de Québec, par Charles-Honoré Laverdière et Henri Raymond Casgrain, Québec, Léger Brousseau, 1871, X-403 p.

JUNEAU, Marcel, "Le vocabulaire maritime dans la langue commune au Québec : apport gallo-roman», dans Canadiana Romanica, Franfais du Canada Franfais de France, vol. 6, 1991, p. 17-23.

JunGl = JUNEAU, Marcel, «Glanures lexicales dans Bellechasse et dans Lévis $»$, dans TraLiQ, vol. 1, 1975, p. 141-191.

JunInv = JUNEAU, Marcel, «Un inventaire de biens québécois de la fin du XVIII ${ }^{e}$ siècle», dans TraLiLi, t. 10, vol. 1, 1972, p. 181-223.

JunJum = JUNEAU, Marcel, La jument qui crotte de l'argent $:$ conte populaire recueilli aux Grandes-Bergeronnes (Québec), édition et étude linguistique, Québec, Les Presses de l'Université Laval, 1976, 143 p.

JunLex = JUNEAU, Marcel, Problèmes de lexicologie québécoise : prolégomènes à un trésor de la langue française au Québec, Québec, Les Presses de l'Université Laval, $1977,278 \mathrm{p}$.

JunPMeun = JUNEAU, Marcel et Claude POIRIER, Le livre de comptes d'un meunier québécois (fin $X V I I^{2}$ - début XVIII siècle), édition avec étude linguistique, Québec, Les Presses de l'Université Laval, 1973, 228 p.

JunPron = JUNEAU, Marcel, Contribution à l'bistoire de la prononciation française au Québec : étude des graphies des documents d'archives, Québec, Les Presses de l'Université Laval, 1972, XVIII-311 p.

Kalm = KaLM, Pehr, Voyage de Pebr Kalm au Canada en 1749, traduction annotée du journal de route par Jacques Rousseau et Guy Béthune avec le concours de Pierre Morisset, Montréal, Pierre Tisseyre, 1977, CLXV-674 p.

Kœssler = K(ESSLER, Maxime, Les faux amis des vocabulaires anglais et américain, Paris, Librairie Vuibert, 1975, $582 \mathrm{p}$.

Lacombe $=$ LACOMBE, Albert, Dictionnaire de la langue des Cris, Montréal, Beauchemin et Valois, 1874, $711 \mathrm{p}$.

LaCurne = SAINTE-PALAYE, Jean-Baptiste de la Curne de, Dictionnaire historique de l'ancien langage franfois ou Glossaire de la langue françoise depuis son origine jusqu'au siècle de Louis XIV, Niort, L. Favre, Éditeur, et Paris, H. Champion, Libraire, 1875-1882, 10 vol. 
LaHontan, Louis-Armand de Lom d'Arce, baron de, Nouveaux voyages de Mr le baron de Labontan dans l'Amerique septentrionale. Qui contiennent une relation des differens Peuples qui y babitent, la nature de leur Gouvernement, leur Commerce, leur Coûtume, leur Religion, Ė leur maniere de faire la Guerre. L'interêt des Franjois et des Anglois dans le Commerce qu'ils font avec ces Nations, l'avantage que l'Angleterre peut retirer dans ce Pais, etant en Guerre avec la France, La Haye, Les Freres L'Honoré, 1704, 280 p.

Langages, Paris, Didier-Larousse, depuis 1966.

LAPIERRE, André, «Le manuel de l'abbé Thomas Maguire et la langue québécoise au XIX ${ }^{\mathrm{e}}$ siècle», dans $R H A F$, vol. 35, $\mathrm{n}^{\circ}$ 3, décembre 1981, p. 337- 354.

LarOrth = Dictionnaire historique de l'orthographe franfaise, sous la direction de Nina Catach, Paris, Larousse, 1995, XL-1327 p.

Laveaux = LAVEAUX, Jean-Charles, Nouveau dictionnaire de la langue francaise, Paris, Deterville, 1820, 2 vol.

Lavoie = LAVOIE, Thomas, Gaston BERGERON et Michelle CÔTÉ, Les parlers franfais de Charlevoix, du Saguenay, du Lac-Saint-Jean et de la Côte-Nord, enquêtes effectuées de 1972 à 1976 et de 1977 à 1980, Gouvernement du Québec, Ministère des Communications, 1985, 5 vol. (Dans les références, Lavoie est suivi du numéro de la question.)

LeclVoit = LeCLERC, Paul-André, Les voitures à chevaux à la campagne, La Pocatière, Musée François-Pilote, 1978, 129 p.

LEFEBVRE, Jean-Jacques, «Études généalogiques. La famille Viger. Le maire Jacques Viger ( +1858$)$. Ses parents - ses ascendants - ses alliésm, dans Mémoires de la Société généalogique canadienne-française, vol. XVII, $\mathrm{n}^{0} 1$, janv.mars 1966, p. 216.

Le français moderne, Paris, Éditions d'Artrey, depuis 1933.

LemVieux = LEMIEUX, Germain, Les vieux m'ont conté, contes franco-ontariens, Montréal, Les Éditions Bellarmin et Paris, Maisonneuve et Larose, 1973 à 1990, 29 t. (Contes recueillis de 1953 à 1969).

LepComp = LEPELLEY, René, «Les comparaisons dans le Val de Saire (BasseNormandie)m, dans $R L i R$, t. 42,1978 , p. 384-418.

LepNorm = LEPELLEY, René, Dictionnaire du français régional de Normandie, Paris, Éditions Bonneton, 1993, $157 \mathrm{p}$.

LepVoc = LEPELLEY, René, Vocabulaire des côtes du département de la Manche : relevé et étymologie des noms des espèces marines, Condé-sur-Noireau, Éditions C. Corlet et Caen, Centre de publication de l'Université de Caen, 1985, 168 p.

LESCARBOT, Marc, Histoire de la Nouvelle-France. Contenant les navigations, découvertes, $\mathcal{G}^{\circ}$ habitations faites par les Franfois és Indes Occidentales \& NouvelleFrance souz l'avceu E authorité de noz Rois Tres-Cbrétiens, E les diverses fortunes d'iceux en l'execution de ces choses, depuis cent ans jusques à bui. En quoy est comprise l'Histoire Morale, Naturelle, E Geographigue de ladite province: Avec les Tables E Figures d'icelle, A Paris, chez Iean Milot, 1609, 888 p.

Lexis = Lexis : dictionnaire de la langue francaise, Paris, Librairie Larousse, 1975, LXXIX-1950 p.

Littré = LITTRÉ, Émile, Dictionnaire de la langue française, Paris, Librairie de L. Hachette et Cie, 1863-1872, 3 vol. 
LittréS = LITTRÉ, Émile, Dictionnaire de la langue franfaise. Supplément suivi du Dictionnaire étymologique des mots d'origine orientale, Paris, Librairie Hachette et Cie, 1877, IV-351 p.

LorBeauce $=$ LORENT, Maurice, Le parler populaire de la Beauce, [Montréal], Les Éditions Leméac, 1977, 225 p.

MCATEE, Waldo Lee, Folk-names of Canadian Birds, National Museum of Canada, $2^{\mathrm{e}}$ éd., Ottawa, bulletin 149, 1959, 74 p.

McDermMiss = McDERMoTT, John Francis, $A$ Glossary of Mississippi Valley French 1673-1850, Saint Louis, Washington University, 1941, 161 p.

Maguire = MAGUIRE, Thomas, Manuel des difficultés les plus communes de la langue franfaise, adapté au jeune âge, et suivi d'un recueil de locutions vicieuses, Québec, Fréchette, 1841, $184 \mathrm{p}$.

MartRech = MARTEL, Pierre, "Recherches sociolinguistiques dans la région de Sherbrooke (Estrie) : présentation et premiers résultats de l'étude lexicale de 16 textes libres», dans TraLiQ, vol. 2, 1978, p. 21-42.

MartVend = MARTEllière, Paul, Glossaire du Vendömois, Orléans, Herluison et Vendôme, Ripé, 1893, 366 p.

Massicotte = MASSICOTTE, Micheline, Le parler rural de l'Île-aux-Grues (Québec). Documents lexicaux, Québec, Les Presses de l'Université Laval, 1978, 554 p. (Enquêtes effectuées en 1971-1972. Dans les références, les chiffres renvoient aux sections.)

Massignon = MASSIGNON, Geneviève, Les parlers français d'Acadie : enquête linguistique, Paris, Librairie C. Klincksieck, 1962, 2 vol. (Enquêtes effectuées entre 1946 et 1950. Dans les références, les numéros renvoient aux questions.)

MassViger = MASSICOTTE, Édouard-Zotique, «Jacques Viger et sa famille», dans $B R H$, t. XXI, n5, 1915, p. 148-149.

Mathews $=A$ Dictionary of Americanisms on Historical Principles, edited by Mitford M. Mathews, Chicago (Illinois), The University of Chicago Press, 1951; $4^{\mathrm{e}}$ éd., $1966,1946 \mathrm{p}$.

MélPoiss = Mélançon, Claude, Les poissons de nos eaux, $4^{\mathrm{e}}$ éd., Ottawa, 1973.

Mémoires de la Société généalogique canadienne-française, périodique trimestriel, Montréal, La Société, dep. janv. 1944.

Ménage = MÉNAGE, Gilles, Dictionnaire étymologique de la langue françoise, Paris, Briasson, 1750, 2 vol.; réimpr. : Slatkine Reprints, Genève, 1973.

MénAng = MÉNIÈRE, Charles, Glossaire angevin étymologique comparé avec différents dialectes, Angers, 1880; réimpr. : Laffitte Reprints, Marseille, 1979.

MERCIER, Louis-Sébastien, Néologie, ou Vocabulaire de mots nouveaux, à renouveler, ou pris dans des acceptions nouvelles, Paris, Moussard, 1801, 2 vol.

MinPhon = MINEAU, Robert, Les vieux parlers poiteritns : histoire, phonétique, grammaire, $2^{\mathrm{e}}$ éd. corr. et augm. d'un vocabulaire comparé des parlers poitevins et québécois, Poitiers, Brissaud, 1986, $373 \mathrm{p}$.

MinPoit = MinEAU, Robert et Lucien RACINOUX, Glossaire des vieux parlers poitevins : recueillis dans le département de la Vienne et lieux voisins, Poitiers, Brissaud, 1981, 564 p.

MoisyNorm = MoISY, Henri, Dictionnaire du patois normand indiquant particulièrement tous les termes de ce patois en usage dans la région centrale de la Normandie, Caen, Henri Delesques, 1887, 716 p. 
Molard = MOLARD, Étienne, Le mauvais langage corrigé, $4^{\mathrm{e}}$ éd., Lyon, Yvemault et Cabin, 1810, XII-288-5 p.

Monet = MONET, Philibert, Inventaire des deus langues, franfoise, et latine : assorti des plus utiles curiositez de l'un E' de l'autre idiome, Lyon, C. Obert, 1636, 990 p.; réimpr. : Slatkine Reprints, Genève, 1973.

Montesson, Charles-Raoul, comte de, Vocabulaire du Haut-Maine, Paris, Paul, $1899,541 \mathrm{p}$.

MorHist $=$ Morin, Marie, Histoire simple et véritable, Les annales de l'Hôtel-Dieu de Montréal, 1659-1725, édition critique par Ghislaine Legendre, Montréal, Les Presses de l'Université de Montréal, 1979, [X]]-XXXV-348 p.

MussSaint $=$ MusseT, Georges, avec la collaboration de Marcel PELLISSON et Charles Vigen, Glossaire des patois et des parlers de l'Aunis et de la Saintonge, La Rochelle, Imprimerie Masson, fils \& Cie, t. 1-3, 1929-1932; t. 4-5, 19381948.

MVictF1 = MARIE-VICTORIN, Flore laurentienne, $2^{e}$ éd. entièrement revue et mise à jour par Ernest Rouleau, Montréal, Les Presses de l'Université de Montréal, $1964,925 \mathrm{p}$.

Nicot $=$ NICOT, Jean, Thresor de la langue franfoyse, tant ancienne que moderne. Auquel entre autres choses sont les mots propres de marine, venerie, Eo faulconnerie, Paris, David Douceur, Libraire, 1606, 966 p.; réimpr. : Éditions du Temps, Paris, 1979; 1621; réimpr. : Éditions A. et J. Picard et Cie, Paris, 1960.

NisÉt = NISARD, Charles, Étude sur le langage populaire ou patois de Paris et de sa banlieue, Paris, Librairie A. Franck, 1872, 454 p.

NisPar = NISARD, Charles, De quelques parisianismes populaires et autres locutions non encore ou plus ou moins imparfaitement expliquées des XVII ${ }^{e}, X V I I I^{r}$ et $X I X^{e}$ siècles, Paris, Maisonneuve \& Cie, Éditeurs, 1876, VII-232 p.

NuTE, Grace Lee, The Voyageur, New York and London, D. Appleton and Company, 1931, 289 p.

Nyrop $=$ NYROP, Kristoffer, Grammaire bistorique de la langue française, $4^{\mathrm{e}}$ édition, Paris-Copenhague, 1914-1960, 6 vol.; réimpr. : Slatkine Reprints, Genève, 1979.

OED = The Oxford English Dictionary, prepared by J. A. Simpson and E. S. C. Weiner, $2^{\text {nd }}$ ed., Oxford, Clarendon Press, New York, Oxford University Press, 1989, 20 vol.

Oudin = OUDIN, Antoine, Curiositez franfoises pour supplement aux dittionnaires ou Recueil de plusieurs belles proprietez, avec une infinité de proverbes $\mathcal{E}$ quolibets, Paris, Antoine de Sommaville, 1640, [V]]-616 p.; réimpr. : Slatkine Reprints, Genève, 1971.

OUELLET, Fernand, «Inventaire de la Saberdache de Jacques Viger», dans $R A P Q$, t. 36-37, 1955-1956 et 1956-1957, p. 33-176.

PARTRIDGE, Eric, A Dictionary of Slang and Unconventional English, $8^{\text {th }}$ ed., New York, Macmillan, 1984, XXIX-1400 p.

PidRom = Pidoux, Edmond, Le langage des Romands, $2^{\mathrm{e}}$ éd., Lausanne, Ensemble, 1984, 173 p.

PierReHUMBert, William, Dictionnaire bistorique du parler neuchatelois et suisse romand, Neuchâtel, Victor Attinger, 1926, 763 p. 
Piggott = Piggott, Glyne Leroy et A. Grafstein, An Ojibwa Lexicon, Ottawa, National Museum of Canada, 1983, IX-377 p.

PLar = Le petit Larousse illustré, Paris, Larousse, 1993, 1784 p.; 1998, 1787 p.

Platt $=$ PLATT, L., Dictionnaire critique et raisonné du langage vicieux ou réputé vicieux; ouvrage pouvant servir de complément au dictionnaire des difficultés de la langue franfaise par Laveaux, Paris, Aimé André, Libraire, 1835, XI-463 p.

PoirAcad = PoIRIER, Pascal, Glossaire acadien, t. 1, Nouveau-Brunswick, Université Saint-Joseph, 1953; t. 2-5, Moncton (N.-B.), Centre d'études acadiennes, Université de Moncton, 1977 (ms. de 1927).

POIRIER, Claude, "Le lexique québécois : son évolution, ses composantes", dans Culture populaire et littératures au Québec, sous la dir. de René Bouchard, Saratoga (California), Anma Libri, 1980, p. 43-80.

PoITEvin, Prosper, Nouveau dictionnaire universel de la langue franfaise, Paris, C. Reinwald, Libraire-Éditeur, 1856, vol. 1; 1860, vol. 2.

PoTIER, Pierre-Philippe, «Façons de parler proverbiales, triviales, figurées, etc., des Canadiens au XVIII sièclem, dans BPFC, vol. III, 1904-1905, p. 213$220 ; 252-255$; 291-293; vol. IV, 1905-1906, p. 29-30; 63-65; 103-104; 146$149 ; 224-226 ; 264-267$.

PPQ $=$ DULONG, Gaston et Gaston BERGERON, Le parler populaire du Québec et de ses régions voisines. Atlas linguistique de l'Est du Canada, gouvernement du Québec, ministère des Communications en coproduction avec l'Office de la langue française, Québec, Éditeur officiel du Québec, 1980, 10 vol. (Enquêtes effectuées de 1969 à 1973. Dans les références, le numéro qui suit le sigle PPQ renvoie au numéro de la question; les lettres $s$ ou $x$ qui accompagnent quelquefois le numéro, proviennent respectivement des rubriques "Réponses complémentaires» et «Vocabulaire de contexte»).

$\mathrm{PRob}=$ Le Nouveau Petit Robert : dictionnaire alphabétique et analogique de la langue franfaise, nouv. éd. remaniée et amplifiée, sous la direction de Josette ReyDebove et Alain Rey, Paris, Dictionnaires Le Robert, 1993, XXXV-2490 p.

ProvFlore $=$ PROVANCHER, Léon, Flore canadienne ou description de toutes les plantes des forêts, champs, jardins et eaux du Canada : donnant le nom botanigue de chacune, ses noms vulgaires francais et anglais, indiquant son parcours géographique, Québec, J. Darveau, 1862, 2 vol. Illustrations.

QUEMADA, Bernard, Matériaux pour l'bistoire du vocabulaire français : datations et documents lexicographiques, $2^{\mathrm{e}}$ série, Paris, Didier, puis C. Klincksieck, depuis 1970.

QuemDict = QUEMADA, Bernard, Les dictionnaires du français moderne, 1539-1863: étude sur leur histoire, leurs types et leurs méthodes, Paris, Didier, 1967, $682 \mathrm{p}$.

QUESNEL, Joseph, «L'Anglomanie ou le Diner à l'angloise, 1802», dans La Barre du jour, vol. 1, $\mathrm{n}^{\mathrm{os}} 3-5$, juill.-déc., 1965, p. 117-141.

Random House Dictionary of the English Language, $2^{\text {nd }}$ ed. unabridged, ed. by Stuart Berg Flexner, New York, Random House, 1987, XIII-2478 p.

$\mathrm{RAPQ}=$ Rapport de l'archiviste de la province de Québec, t. 1-40, 1920-1960; Rapport des Archives du Québec, à partir du t. 41, 1963.

ReadLouis = READ, William A., Louisiana-French, Baton Rouge, Louisiana State University Press, 1931, XXIV-253 p. 
ReyDAngl = REY-DEBOVE, Josette et Gilberte GAGNON, Dictionnaire des anglicismes: les mots anglais et américains en français, collection "Les usuels», Paris, Le Robert, 1980, XIX-1152 p.

ReyExpr = REY, Alain et Sophie CHANTREAU, Dictionnaire des expressions et locutions, collection «Les usuels», $2^{e}$ éd., mise à jour, Paris, Dictionnaires Le Robert, 1993, XV-888 p.

ReyHist = Dictionnaire historique de la langue francaise, sous la direction de Alain Rey, Paris, Dictionnaires Le Robert, 1992, 2 vol., 2383 p.

RézOuest = RÉzEAU, Pierre, Dictionnaire des régionalismes de l'Ouest entre Loire et Gironde, Les Sables-d'Olonne, Le Cercle d'or, 1984, 302 p.

RézVend = RÉzEAU, Pierre, Un patois de Vendée $:$ le parler rural de Vouvant, Paris, Klincksieck, 1976, 352 p. Illustrations.

RézVoc = RÉzEAU, Pierre, Le «Vocabulaire poitevin* (1808-1825) de Lubin Mauduyt, Tübingen, Max Niemeyer Verlag, 1994, 368 p.

RHAF = Revue d'bistoire de l'Amérique franfaise, trimestriel, Montréal, Institut d'histoire de l'Amérique française, depuis 1947.

Richelet $=$ RICHELET, Pierre, Dictionnaire françois, contenant les mots et les choses, plusieurs nouvelles remarques sur la langue françoise : ses Expressions Propres, Figurées E̊ Burlesques, la Prononciation des Mots les plus difficiles, le Genre des Noms, le Regime des Verbes: Avec les Termes les plus connus des Arts $\mathcal{E}^{\circ}$ des Sciences. Le tout tiré de l'usage et des bons auteurs de la langue franfoise, Genève, Jean Herman Widerhold, 1680, 2 vol.; réimpr. : Slatkine Reprints, Genève, 1970.

RIVARD, Adjutor, «La francisation des mots anglais», dans BPFC, vol. V, 19061907, p. 252-264.

$\mathrm{RJ}=$ The Jesuit Relations and Allied Documents : Travels and Explorations of the Jesuit Missionaries in New France, 1610-1791, ed. by Reuben Gold Thwaites, Cleveland, Burrows Brothers Company, 1896-1901, 73 vol.; réimpr. : Pageant Book Company, New York, 1959, 36 vol.

$\mathrm{RLiR}=$ Revue de linguistique romane, trimestriel, Paris, Société de linguistique romane, depuis 1925 .

RobNorm = ROBIN, Paul-Eugène, et coll., Dictionnaire du patois normand en usage dans le département de l'Eure, Évreux, Imprimerie C. Hérissey, 1879-1882, 2 vol.; réimpr. : Laffitte Reprints, Marseille, 1978, XXIV-458 p.

RobViger = ROBERT, Jean-Claude, "Jacques Vigem, dans $D B C$, vol. VIII, de 1851 à 1860,1985 , p. 1010-1015.

Rohlfs = RoHLFs, Gerhard, Le gascon : études de philologie pyrénéenne, $2^{\mathrm{e}}$ éd., Tübingen, Max Niemeyer Verlag, 1970, 248 p.

RollFaune = Rolland, Eugène, Faune populaire de la France. Noms vulgaires, dictons, proverbes, légendes, contes et superstitions, Paris, Maisonneuve \& $\mathrm{Cie}$, 1877-1911, 13 t. en 6 vol.; réimpr. : G.-P. Maisonneuve et Larose, Paris, 1967,13 t. en 7 vol.

RollFlore = ROLLAND, Eugène, Flore populaire ou bistoire naturelle des plantes dans leurs rapports avec la linguistique et le folklore, Paris, Librairie Rolland, 18961914, 11 t. en 8 vol. ; réimpr. : G.-P. Maisonneuve et Larose, Paris, 1967, 11 t. en 6 vol.

RossPron $=$ RosseT, Théodore, Les origines de la prononciation moderne étudiées au $X V T T^{e}$ siècle d'après les remarques des grammairiens et les textes en patois de la banlieue parisienne, Paris, Colin, 1911, 421 p. 
RoussAnnedda = ROUSSEAU, Jacques, «L'annedda et l'arbre de vie», dans $R H A F$, t. VIII, $\mathrm{n}^{\circ} 1$, juin 1954, p. 171-212.

RoussBouch $=$ RousSEAU, Jacques, $«$ Pierre Boucher, naturaliste et géographe», dans BouchHist, 1964, p. 262-401.

RoussP1 = RoussEAU, Jacques, "Les noms populaires des plantes au Canada français», dans Études sur le parler franfais au Canada, 1955, p. 135-173.

RoY, Camille, Historiens de chez nous : études extraites des essais et nouveaux essais sur la littérature canadienne, Montréal, Beauchemin, $1935,190 \mathrm{p}$.

RoyViger = RoY, Camille, «Jacques Viger», dans BPFC, vol. VIII, 1909-1910, p. 4255 ; également dans Historiens de chez nous (du même auteur), 1935, p. 67-83.

Sagard = SAGARD, Gabriel Théodat, Le grand voyage du pays des Hurons, situé en l'Amerique vers la Mer douce, ès derniers confins de la Nouvelle France, dite Canada. Avec un Dictionnaire de la Langue Huronne pour la commodite de ceux qui ont à voyager dans le pays, E' n'ont l'intelligence d'icelle langue, Paris, Denys Moreau, 1632, [24]-380 p.

Sainéan = SAINÉAN, Lazare, Le langage parisien au XIX $X^{e}$ siècle, Paris, E. de Boccard, 1920, XVI-590 p.

SimTour = Simon, Jean-Pascal et Marie-Rose Simoni-Aurembou, Dictionnaire du français régional de Touraine, Paris, Éditions Bonneton, 1995, 158 p.

STAPFER, Paul, Récréations grammaticales et littéraires, $2^{\mathrm{e}}$ éd., Paris, Librairie Armand Colin, 1910, 285 p.

TAVERDET, Gérard et Françoise DuMAS, Anthologie des expressions en Bourgogne, Marseille, Rivages, 1984, $177 \mathrm{p}$.

THUROT, Charles, De la prononciation franfaise depuis le commencement du XVT' siècle, d'après les témoignages des grammairiens, Paris, Imprimerie nationale, vol.1, 1881, et vol 2, 1883.

$T L F=$ Trésor de la langue franfaise. Dictionnaire de la langue du XIX et du XX $X^{e}$ siècle (1789-1960), sous la dir. de Paul Imbs, Paris, Éditions du Centre national de la recherche scientifique, 1971-1994, 16 vol.

TraLiLi = Travaux de linguistique et de littérature, publ. par le Centre de philologie et de littératures romanes de l'Université de Strasbourg, Paris, C. Klincksieck, depuis 1963.

TraLiQ = Travaux de linguistique québécoise, vol. 1, publ. par Marcel Juneau et Georges Straka, Québec, Les Presses de l'Université Laval, 1975, 355 p.; vol. 2, publ. par Lionel Boisvert, Marcel Juneau et Claude Poirier, 1978, VIII201 p.; vol. 3, 1979, 327 p.; vol. 4, publ. par Jean-Marcel Léard, 1983, 251 p.

Trévoux $=$ TrÉvoux, Dictionnaire universel franfois et latin, Paris, Estienne Ganeau, 1704, 3 vol.; $2^{e}$ éd., Paris, Florentin Delaulne et autres, 1721, 5 vol.; $3^{\complement e ́ d ., ~ P a r i s, ~ J u l i e n-M i c h e l ~ G a n d o u i n, ~ 1732, ~} 5$ vol.; $5^{\mathrm{e}}$ éd., Paris, Compagnie des libraires associés, 1752,8 vol.; $6^{e}$ éd., 1771,8 vol.

Troyes $=$ Troyes, Pierre, chevalier de, Journal de l'expédition du chevalier de Troyes à la baie d'Hudson, en 1686, édité et annoté par Ivanhoë Caron, Beauceville, La Compagnie de l'Éclaireur, 1918, IX-136 p.

VALMONT DE BOMARE, Jacques-Christophe, Dictionnaire raisonné universel d'histoire naturelle : contenant l'bistoire des animaux, des végétaux et des minéraux, avec l'bistoire et la description des drogues simples tirées des trois règnes, Paris, Didot, 1764, 5 vol. 
VassPic = VASSEUR, Gaston, Dictionnaire des parlers picards du Vimeu (Somme) avec considération spéciale du dialecte de Nibas, Amiens, Musée de Picardie, 1963, $690 \mathrm{p}$.

Vaugelas = VAUGELAS, Claude Favre de, Remarques sur la langue franfoise utiles à ceux qui veulent bien parler et bien escrire, Paris, Augustin Courbé, 1647, 615 p.

VerrAnj = VERRIER, A.-J. et R. ONILLON, Glossaire etymologique et historique des patois et des parlers de l'Anjou, Angers, Germain et G. Grassin, 1908, 2 vol., XXXII-528 et $587 \mathrm{p}$.

VIGER, Jacques, «Néologie canadienne, ou Dictionnaire des mots créés en Canada \& maintenant en vogue; - des mots dont la prononciation \& l'ortographe sont différentes de la prononciation \& ortographe françoises, quoique employés dans une acception semblable ou contraire; et des mots étrangers qui se sont glissés dans notre langue», dans $B P F C$, vol. VIII, 1909-1910, p. 101-103; 141-144; 183-186; 234-236; 259-263; 295-298; 339-342 (ms. de 1810).

Volney $=$ VOLNEY, Constantin-François de Chassebœuf, comte de, Tableau du climat et du sol des Etats-Unis d'Amérique. Suivi d'éclaircissemens sur la Floride, sur la colonie Franfaise au Scioto, sur quelques colonies Canadiennes et sur les Sauvages, Paris, Courcier, Imprimeur-Libraire et Dentu, ImprimeurLibraire, 1803, 2 vol.

VurpBeauj = VURPAS, Anne-Marie et Claude MiCHeL, Dictionnaire du franfais régional du Beaujolais, Paris, Éditions Bonneton, 1992, 191 p.

WALlOT, Jean-Pierre, «Ross Cuthbert», dans DBC, vol. IX, de 1861 à 1870, 1977, p. 206-207.

WARTBURG, Walther von, Bibliographie des dictionnaires patois, Paris, Librairie É. Droz, 1934, $146 \mathrm{p}$.

Webster $=$ Webster's Third New International Dictionary of the English Language Unabridged, Editor in chief : Philip Babcock Gove and the Merriam-Webster editorial staff, Springfield (Mass.), G. \& C. Merriam Co., 1981, 2662 p.

WOLF, Lothar, «Le langage de la Cour et le français canadien. Exemples de morphologie et de syntaxe», dans Canadiana Romanica, Français du Canada Français de France, vol. 6, 1991, p. 115-123.

YON, Armand, L'abbé H.-A. Verreau : éducateur, polémiste, historien, Montréal, Fides, 1946, 208 p. 
This page intentionally left blank 


\section{INDEX LEXICAL}

L'Index contient tous les termes présentés en vedette dans les manuscrits de Viger, ainsi que tous les mots cachés. Y figurent, en outre, tous les termes qui, comme équivalents ou synonymes des lexies étudiées, présentent un intérêt lexicologique dans le cadre de notre étude. Un certain nombre d'entre eux ont fait l'objet de commentaires dans l'étude linguistique. On trouvera également dans cet Index les mots et les expressions qui figurent dans les listes données dans les Appendices.

abât de neige, de pluie $23,27,28,41$, $100,133,156,241,266$; (sous bordée) 44,102

âbre $23,25,41,133,141,156,249$

abriller v. tr. et pron. 245

achigan 16, 43, 232, 246

acquet (avoir plus d' ) 42, 157; (sous quitte) 82,120

affaire (avoir de qqn) 42, 154, 157

affairé (être ) 257, 265

agriculteur (sous habitant) 69, 113; 261

ahuri 259

ahurissant, ante adj. et nom 41,99 , $157,241,249$

aimable n. masc. «mondain» (sous désapointer) 55; 157

aimer qqch. comme la colique 252

Allemand (avoir, être une tête d' ) 42, 157, 261

aller voir la créature (sous créature) 54 , 182

aller voir sa blonde (sous blonde) 48 , 164,263 allumer v. intr. $40,99,157,241,248$, 260,266

amanché (être mal ) 263

amancher $23,40,99,151,158,248$, 259 ; (sous ramancher) 120

amant (sous cavalier) 53, 104, 178

amante (sous blonde) 48, 164; (sous cavalier) 178

amarrer 28, 40, 158, 248

âme (avoir 1' clouée, rivée dans le corps) 258

amidon 41; (sous empois) 57, 109, 249

amis (être jusqu'au plat) 256

amont la côte $28,41,154,158,248$

amour (faire l' ) (sous farôder) 64, 193

amoureux comme une chatte 262

amoureux de 36 blondes (sous blonde) 48,164

apichimon $16,42,100,232,247,250$, 259,266

appâter 255

appeler $\left(s^{\prime} \sim\right)$ «se nommer» 258 
appeler (s' joli-cœur) (sous joli-ccour)

$28,71,115,203,265$

appointement $26,40,100,235$

appointer 40, 100, 235, 248, 266

après (s'agripper qqch.) 252

après-dinée (sous plaisant) 81, 119;

(sous relevée) 82, 120; 158

araignée n. masc. $23,42,147,158$, 249

arbre de vie (sous épinette) 62, 189, 246

arbre du paradis (sous épinette) 62, 189,246

argent n. fém. 23, 41, 146, 158, 249

argent blanche 258

argent sous le pouce 255

arignée $42,136,158$

arracheur de dents (mentir comme un ) 260

arrêter qqch. 250

arriette (une belle ) 265

arse $42,100,134,158,265$

assemblée (Chambre d' ) (sous payer) 78,118

astigoiner 255

a'tic "caribou" 246, 247

attiffer (s' ) 255

attisée $17,19,20,43,159,256$

aveindre 251

avisse $40,144,159,248$

avoine (manger de l' ) 245, 259, 263

awapou 247

babines (flanquer un tapin par les ) 260

bâclée (affaire ) 254

bagage 49, 159; (sous drigaille) 57

baggatiway 248

bagoulard 264

bâiller le bec 265

baise mon pouce (être comme ) 252 baiser «tromper» 47, 159, 241

baiser en guedou «tromper" (sous baiser) 47,159 ; (sous guedou) 67

baiser en pincettes «tromper» (sous baiser) 47, 159; (sous pincettes) $81 ; 259$

baiser en pincettes «embrasser» (sous baiser) 47; (sous pincettes) 214

balancer (se $\sim$ ) (sous berlanciller) 44, $101,161,162$

balançoire (sous berlancille) 44, 101, 161

balayer 249

balier $44,152,160,264$

balle 250

banal n. masc. 45, 160, 250

banc-lit 261

bandon n. fém. 45, 160, 250

bandon (avoir, donner ) 45, 160

baragouin (sous micmac) 73; (sous ratapiat) 84

barbeau «insecte» 251

barbes (s'en licher les $\sim$ ) (sous licher) 71,115

barbots 254

barbots (faire des ) 254

Barrabbas (connu comme $\sim$ a la passion) 261

bas (débouler en $\sim$ de) 252

Basque (être un malin ) 46, 160, 251

Basque (malin comme un ) 46, 160

Bastonnois 247

batailleur, euse 45,250

batelée 265

batons de traine (avoir les jambes comme des ) 253

battoir (sous pelote) 80; 213

baudrier (sous belt) 47

bavasser $26,48,160,253,265$

beau comme un fâro de campagne (sous faro) 64, 111, 192 
beauté (une ) 48, 161, 265

bec-de-grue (sous gérémium) 67, 198

béguer 252

béguer (ne pas pour dire qqch.) 252

Bejin (dire à la $\sim$ qu'elle te peigne) 263

belette (passer en ) 256

belle (la manquer ) (sous manquer) 75,205

belt $47,235,251$

bèr $26,45,161$

berdas 143,250

berdasser 143, 250

berlancille 44, 101, 135, 161, 248

berlanciller $44,101,135,153,161$, 248

berline $27,43,162$

berloque $45,143,162,249$

berre 249

bête à la grande queue 256

bête-puante 251

bête puante (lever le cul comme une ) 252

bètot 254

beurrée $27,48,103,162,263,266$

beurrer 17, 19, 48, 103, 162, 263

beurrer (se les mains) 48, 103, 163

bicler 45, 102, 163, 249

bicleux, euse adj. et nom $27,45,102$, $150,163,264$

bière (n'être pas de la petite ) 255

bill 251

biner 46, 163, 245, 250; (sous corner) 52

blague 265

blague (fin-fin la ) 256

blancs (avoir les quatre pieds ) «être libre» 256

blancs (faire de cinq sols six ) 265 bled d'Inde (sous coton) 54; (sous épiochon) 59 ; (sous sucet) 87; 163

bled d'Inde (avoir, donner un beau $\sim$ ) (sous coton) 54 ; (sous bled d'Inde) 163

bled d'Inde (sec comme un coton de ) (sous coton) 54; 264

bled dinde (éplucher du ) 264

bleds (couper les $\sim$ ) (sous couper) 51

bleds (scier les $\sim$ ) (sous couper) 51; 224

blême comme un linge 261

bleuet $49,163,246$

blonde 48, 164, 241

blonde (aller voir sa ) 48, 164, 263

blonde (fort sur la ) 48, 164

blondes (amoureux de $36 \sim$ ) 48, 164

blouser (se ) 261

bœuf (robe de ) 82, 222, 249

bœuf (soulier de $\sim$ ) (sous soulier) 86, $121,225,250,261$

boufs (mettre la charrue devant les $\sim$ ) 245

boire à la grande tasse 256

boire comme un trou 260

bois (coureur des ) 247

bois de requise 253

bois (donner le $\sim$ pour les écopeaux) 253

bolle 249

bombarde 27, 44, 164, 261; (sous trompe) 88

bombe $23,27,28,43,101,164,248$, 260, 266; (sous canard) 49

bonnet blanc et blanc $\sim 258$

bord 45, 165, 250, 254

bordée (de neige) 27, 44, 102, 165 , 249; (sous abât) 41, 100; (sous giboulée) 112

bordel $27,43,165$

borgne 250 
boucane $23,43,101,166,248,266$

boucaner (de la viande, etc.) 23, 101, 166

boucaner «dégager de la fumée» 43, 101, 166

Bouche (parler comme la $\sim$ ) 260

boudin 250

boudin (faire du ) 48, 166, 241, 262

bouette 255

bougon $22,27,45,47,166,250,262$

bougonner 254

bougre à bougre 262

bougrine 261

bouillir 258

bouillir (faire la marmite) 258

bouilloire 27, 28, 266; (sous bombe) 43, 101, 165; (sous canard) 49, 174

boule (tête de $\sim$ ) (sous tête) 90, 227, 247,265

bouler qqn 258

bouquin «embouchure d'une pipe " $44,167,241,249,260$

bourasse $46,139,167$

bourasser $46,167,250$

bourasseur, euse 25, 46, 150, 167

bourdignon 262

Bourgogne «Burgoyne» (sous bourgogner) 43

bourgogner 13, 43, 235

bourguignon 44, 167, 249

bout d'homme 27; (sous bougon) 48; (sous nijon) 76, 117; 245

bouts-de-canot 247

boyard $44,137,168,248$

brailler comme un veau 252

bran de scie 248

brancard «civière» (sous boyard) 44; 248 brancard «menoirs» 44; (sous travail) 88

brandiller (se $\sim$ ) (sous berlanciller) 44, $101,161,162 ; 248$

brandilloire (sous berlancille) 44, 101, $161 ; 248$

branler dans le manche 245

braque (fou comme ) 255

brasse (faire une sottise de $\sim$ ) 255

brâsser (le poële, le chaudron, etc.) $23,26,46,102,133,168,259$

brâsser "réprimander" $46,102,168$, 262

braye $47,135,144,168$

brayer (du chanvre, du lin) 47, 135 , 168,251

bredas «remue-ménage * $16,47,143$, 169

bredas (faire le $\sim$ ) «faire la lessive, le blanchissage, le lavage du linge» $47,143,169,260$

bredasser v. tr. et intr. $16,47,143$, 169,260

bredasserie $47,149,170$

bredassier, ière $47,150,170$

brigade 247

Brillant (prendre du café comme la ) 49,170

brimbale 249

brin 250

brique de savon 255,265

brocanter 264

brouscailler v. tr. et pron. 46, 138 , $152,170,250$

brousquailler qqn 260

brousse-poil (à ) 85, 144, 170, 220, 257,265

brun (faire ) 49, 170, 257

brunante $26,49,149,171$

brunante (à la ) 49, 171, 257

brune (sous brunante) 49, 171 
brune (sur la $\sim$ ) (sous brunante) 49, 171

bûche (être une ) 262

buffet 45, 171, 249

buffle (sous robe de bocuf) 82, 223

Burgoyne 13; (sous bourgogner) 43, 235

busc (courir au ) 252

butard 251

butin $23,44,101,171,248,263,264$; (sous drigaille) 57; (sous gaspiller) 68

cabaret (sous cantine) 53, 104, 176

cabaretier, ière (sous cantinier) 53, $104,105,176$

cabriolet 248

cabrouet 26, 50, 171, 248

câdre 50, 105, 133, 172, 249, 254

café (prendre du $\sim$ comme la Brillant) 49,170

cage $27,52,172$

cahot $26,50,172,260$

cahots (abattre les ) 51, 173

cajeux 27, 52, 173

calèche (sous brancard) 44; (sous débarquer) 55, 106; (sous ramancher) 82,$120 ; 173$

calumet (pierre à ) 246, 247

camper 247

canadien, ienne nom et adj. (sous arse) 100; (sous bourgogner) 43; (sous brâsser) 102; (sous butin) 101; (sous cantine) 104; (sous cantinier) 53; (sous cantinière) 105 ; (sous ramancher) 82; (sous traineau) $88 ; 173$

canard $27,28,49,174,248,260,266$;

(sous bombe) 43, 101

canard (un beau ) 252

cannevette (sous cantine) 53, 104; 174 canot (sous canotée) 54, 105; (sous canoter) $53 ; 174$

canot d'écorce (sous canotée) 105; 174,247

canotée $18,23,54,105,149,175$

canoter $53,105,175,241,263$

canoteur, euse 54, 105, 175

Cantin (la chienne à $~$ lui monte sur le dos) 260

cantine epetit coffre» $26,53,104,175$

cantine «cabaret, taverne» 53, 104, 175,263

cantinier, ière 53, 104, 105, 176

capuche 52, 103, 176, 250, 261, 266

carcajou 246

caribou 16, 26, 50, 233, 246, 247, 249

cariole 27, 50, 176, 248, 259; (sous berline, bordel) 43; (sous robe de bceuf) 82; (sous travait) 88

carotte à Moreau 254

cartron 23, 51, 142, 177, 249

casque 52,177

casseau $52,177,250$

cassette "petit coffre" (sous embarquer) 57,109

castonade $50,143,177$

cause (à $\sim$ donc) 255

cavalier $53,104,177,263$

cèdre «thuya occidentalis » 247

celle-ci, celle-là 250

chambre (sous espérer) 58; 178

Chambre d'assemblée (sous payer) 78 , 118

chance 250

chanceux 250

chandelle (sous tuer) 87

chandelle (brûler la par les deux bouts) 263

chandelle (éviter une belle ) 54, 178 changé (être ) «pâle, défait» 255 
chanter comme un perdu 257

chanter comme un rêve 252

chardron $23,50,142,178,248$

charger $50,178,248$

charretée (sous voyage) 90, 124, 232

charrue (mettre la $\sim$ devant les bœufs) 245

chat échaudé craint l'eau froide 245, 259

chat sauvage 249

chat sauvage (courir comme un $\sim$ ) 254

chaton(n)er 53, 104, 178

chatte (amoureux comme une $\sim) 262$

chatter (sous chaton(n)er) 53, 104, 178

chaud (vite pendant que c'est $\sim$ ) 253

chaudière (faire ) 247

chaudière (faire à part) 262

chaume 250

chauve-souris 250

chemin (faire passer qqn par un où il n'y aura pas de pierres) 262

chemin uni comme un chemin de cartes 261

chemise (devoir plus à sa peau qu'à sa ) 254

cheniquer 51, 236, 249

cheniqueur, euse 52, 149, 236

chétif (un ) 253

cheval (dur comme un $\sim$ ) 253

chèvre (curieux comme la $\sim$ à Jacques

Cartier) 257

chez nous 250

chien couchant (faire le $~ 255$

chien de plomb (leste comme un $\sim$ ) 245,261

chien et loup (entre $\sim$ ) (sous brunante) 49,257

chien (garder un $\sim$ de sa chienne) 258

chien (malade comme un $\sim) 256$ chien (marcher comme un $\sim$ qui vient des vêpres) 252

chien (ne pas valoir les quatre fers d'un ) 261

chien (reçu comme un $\sim$ à vêpres) 260 chien (reçu comme un $\sim$ dans un jeu de quilles) 260

chienne (la $\sim$ à Cantin lui monte sur le dos) 260

chienner (sous chienneter) 52, 104, 179,266

chienneter 52, 104, 152, 179, 266

chiens (les gros $\sim$ ne mordent pas) 253

chiens (temps à ne pas mettre les dehors) 261

chiffonner 256

chipoter 254

chopine (payer ) (sous houiller) 113

chou (haut comme un $\sim) 252$

civière 54 ; (sous boyard) 44

clairon «aurore boréale» 54,179

claque (sous mèche) 116; 179

clisse «éclisse» (sous traine) 88, 229, 248,264

clisse (traîne de ) 88, 229, 248, 264

cochonner qqch. 263

coendou (sous port-épic) 80, 216

col noir "cravate» 262

colique (aimer qqch. comme la ) 252

comfortable 27, 52, 103, 236

confessionnals $23,54,146,179,257$

confortable 248

confortatif (sous comfortable) 52, 103; 179

connaittre qqch. comme sa poche 263 connu comme Barrabbas a la passion 261

coppre 54, 237, 263

coque (avoir un ceil à la $\sim$ et l'autre à la mouillette) 245 
cor (à et à crì) 257

corde (avoir de la $\sim$ de pendu) 263 cordeaux 27, 49, 105, 179, 248, 251 corner «enrager» $52,180,245,250$ corner efrapper de ses cornes" (sous ébrayer) 60,$108 ; 180$

cornichon 250

corporal 51, 145, 180, 249

corps vide comme un fanal 265

côte $22,23,51,53,104,180,181,249$

côte (amont la ) (sous amont) 28, 41, $154,158,248$

côte (grand' ) (sous côte) 53, 104

côtes (coureur de $\sim$ ) (sous côte) 104, 181

côtes (courir les ) (sous côte) 53, 104, 181, 245, 257

coton «épi de bled d'Inde dégarni de ses grains" 54, 181

coton «tige de bled d'Inde» (sous sucet) $87 ; 181$

coton de bled d'Inde (sec comme un ) (sous coton) $54 ; 264$

couche chaude 250

couette n. fém. "queue de cheveux" $50,103,181,248,261$

couette n. masc. «lit de plume" 50 , $103,182,248$

couguar 246,247

couler comme un sas 254

coup de terre (sous retontir) 84

coup (faire ) 50, 63, 182, 248

couper le sifflet 256

couper les bleds 51

coureur de côtes (sous côte) 104, 181

coureur des bois 247

courir au busc 252

courir comme un chat sauvage 254

courir la galipote (sous galipote) 67, 197,263 courir le gallop 252

courir les côtes (sous côte) 53, 104, $181,245,257$

couronel $51,137,141,182,249$

courroies 27, 49, 105, 180, 248, 251

cracher (avoir une mine à $\sim$ dessus) 254

cracher sur les tisons 245

crapaud (écrapoutiller, écrapoutir qqn comme un $\sim$ ) (sous écrapoutiller) $61,108,187$

créature 54,182

créature (aller voir la ) 54, 182

créature (fréquenter la ) 54, 182

Cree ou Cris (malin comme un $~$ ) 52 , 103, 183

cri (à cor et à ) 257

crible $27,52,237,251$

crier comme un sourd 257

criquet 251

Cris (v. Cree)

cristail 256

croc au sel (manger à la ) 245

crocheter des pois $51,183,249,260$

crocheteur 51,183

crocs «moustache» ; «dents» 254

crosse (jeu de la ) 247, 248

croûtes (avoir (bien) des à manger) 245,265

cuiller "cueillir" 262

cuire au four de qqn 258

cul (ne pas valoir son plein d'eau chaude) 265

culasse 251

culotte (porter la $\sim$ ) 255

culottes (ramer sans $\sim$ ) 245

curieux comme la chèvre à Jacques Cartier 257

dans (mettre ses bas $\sim$ ses jambes) 255 
dans (mettre ses souliers $\sim$ ses pieds) 255

dans (se mettre $\sim$ son lit) 255

débarbouiller (se ) 263

débarquer 23, 27, 55, 106, 183, 248, 259

déboutonner (se ) 57, 256

débrailler 251

décanillé, ée 57

décaniller $56,107,251$

décent, ente $56,107,237,249$

décharge 247

dégelée «dégel» 26, 56, 183

dégelée (donner une $\sim$ ) (sous dégelée) 56

dégelée (fricasser une $~$ ) (sous fricasser) 64,197

dégobillage 56, 107, 184

dégobiller (sous dégobillage) 56, 107, 262

dégobillis (sous dégobillage) 56, 107 ; 184

dégradé «arrêté» 258

dégrader qqn «le laisser en arrière» 251,258

délivrer les lettres 251

déluge (après moi le ) 256

démancher $23,26,27,55,106,184$, 248,259

démonté 261

denier «monnaie française» (sous coppre) 54

dent (ne pas en avoir pour une creuse ح) 265

dents (faire grincher les ) 253

dents (gricher les (des) ) (sous gricher) 69, 200

déplanter qqch. 252

derrière (se lever le $\sim$ le premier) 254

désap(p)ointer 55, 105, 237, 248, 259

désargenter 265 désoublier 254

détailleur (sous cantinier) 53, 176

deusse 23, 56, 140, 184, 250

déventes 253

dévergondée 251, 255

dévider 251

diable (farine $d u \sim$ retourne en son) 262

diable (malin comme sept fois le $\sim$ ) 255

diable (n'être pas le $\sim$ ) «pas difficile» 255

diable (que le $\sim$ ) «en diable» 257,258

dinde (jouqué comme un ) 253

dirigé, ée (être de) 56, 107, 238

diriger 250

docteur en souppe salée 257

donner (en sur les épicailles) (sous épicailles) 61, 189,263

donner une dégelée 56

donner une ramasse 84

doré (poisson ) 81, 216, 246, 247

dormir comme une soupe (sous soupe) 87,225

douilleter qqn 260

dram $27,56,238$

dresser 251

drette (un mal a ) 257

drigaille 16, 57, 184

driller 251

droit comme un piquet 263

drôlesse 263

dur comme un cheval 253

eau (être plein $d^{\prime} \sim$ ) 254

eau (faire de 1' $) 247$

eau (nager à grande ) 256

ébaroui, ie (sous s'ébarouir) 61, 185

ébarouir (s' ) 61, 184, 251

éborgner 62 
ébourifler 251

ébrâillé, ée (sous ébrâiller) 60, 110, 185

ébraillée n. fém. (sous ébrâiller) 60 , 110,185

ébrâiller v. pron. $17,23,60,110,133$, $151,185,251$

ébrayer $16,60,108,185$

ébrèché 255

écarquiller (sous s'éjârer) 61; 185

écartiller (sous s'éjârer) $61 ; 186$

éclopé 251

écœurant, ante adj. et nom 59,109 , $186,241,261$

écœuré, ée adj. et nom (sous écourer) $59,109,186$

écœurer 59, 109, 186, 241, 250

écolté, ée (sous écolter) 59, 109

écolter v. tr. et pron. 59, 109, 151

écopeau 26, 59, 144, 186, 250, 262

écopeau (sec comme un ) 59, 186

écopeaux (donner le bois pour les $\sim$ ) 253

Écossois (fier comme un ) 260

Écossois (galeux comme un $\sim$ ) 60 , 186

écrapoutillé, ée (sous écrapoutir, écrapoutiller) $61,108,187$

écrapoutiller v. tr. et pron. 61,108 , 153,187

écrapoutiller qqn comme un crapaud $61,108,187$

écrapoutiner 264

écrapoutir v. tr. et pron. $26,61,108$, 187

écrapoutir qqn comme un crapaud 61,108

écroi $58,187,249$

égrafignure (sous graffigner) $68 ; 187$

égratigner (sous graffigner) 68

égratignure (sous graffigner) 68 éjârer (s' ) 61, 133, 188, 264

élan (sous orignal) 77, 118, 246, 247

élingué «étiré» 253

embardée (prendre une belle ) 255

embarquer $57,109,188,248,259$

embarrasser 250

emberlicotter qqn 250, 261

emmancher 248

empêtrer 251

empocheter 61, 152, 188, 265

empois $57,109,188,249$; (sous amidon) 41

empoisonner «empester» 254

ençà $59,188,250$

en Canada (dans le titre) 39; (sous ginseng) 69, 112; (sous travail) 88; (sous office) 118; (sous tuer) $122 ; 155$

ennuyant (sous aburissant) 41, 99; 188

envarié, ée $58,148,189$

épais (un saint ) 262

éparpiller qqch. 253

épaule (regarder par-dessus l' ) 264

épicailles (en donner sur les ) 61, 189,263

épilepsie (sous mal) 75; 251

épinette 62, 189, 246

épiochon 59, 189, 250

éplucher du bled dinde 264

épotraillé $62,138,148,190$

épouffer (s' ) «s'échapper» 257

équilibre 60

équipage 62,190

équipé (mal ) (sous équiper) 61, 190, 257,265

équipement 247

équiper qqn 61, 190

équiper (se faire ) 61

escabeau $26,59,190,241,250$ 
escargot (tête d' ) (sous tête) 90, 190, 265

escarpolette (sous berlancille) 44, 101, 161

esclave 247

escolter 59, 151, 251

escousse 249,258

espèce (un de) (sous matelat) 74

espérer «attendre, patienter» 19,26 , $58,190,249,260$

essayer à 253

estomac "poitrine "; "seins" (sous écolter) 59, 109; (sous ébrâiller) 60, 110 ; (sous pesant) 81; 191

estropié, ée (sous équiper) 61

estropier (s' ) 253

étage 250

étage (premier ) 58, 191

étaim fine 252

étal 250

étau «étal» 59, 149, 191

étau "étoc" 256

étaux pl. (sous étau) 59, 146

étriper qqn 255

étrivant, ante adj. et nom $20,60,108$, 148,191

étriver qqn $20,60,108,191,251$

étriver (faire qqn) 20,60, 108, 191, 255

exemple (par ) 23, 60, 108, 259

factieux 253

factionnaire 63,248

faire chaudière 247

faire chaudière à part 262

faire coup 50, 63, 182, 248

fanal (corps vide comme un ) 265

fanals $23,28,66,146,192,257$

faner (sous foin) 64, 192

fanfan (sous moucher) 74; 192

fard $26,28,63,192$ farine du diable retourne en son 262

farine (remettre sur ) 245

farro $64,111,192,259$

farro (beau comme un $\sim$ de campagne) (sous faro) 64, 111, 192

farôder 64, 111, 193

fart 249

faucille 249

fectif, ive 65,193

femme (parler comme une ) 256

fendre des éclats 258

fener (sous foin) 64, 192, 193, 251

fenouil n. fém. 23, 63, 110, 146, 193, 246, 249

fenouillette $23,63,110,111,193,246$

fer à flasquer (sous flasquer) 62, 110, 196

ferlassement 66, 193

ferlasser $66,143,193,258$

fers (ne pas valoir les quatre $\sim$ d'un chien) 261

fesser 26, 27, 63, 110, 193

fictif, ive adj. 251

fier comme un Écossois 260

fier (se ) 250

fièrement $64,194,261$

fièvres (trembler les $~ 262$

fignoler 65, 111, 194, 263

fignoleur 25, 65, 111, 194

filer doux 254

filer dru 254

fille (sous amancher) 40; (sous balier) 44; 194

fille publique (sous ébrâiller) 60,110; (sous fille) 194

fin (tout seul) 257

fine-boutique $22,23,64,65,111$, 194, 251, 262

fin-fin la Blague 256

finir avec qqch. 65, 238 
finition 22, 112, 195

fiston 252

flambe $25,65,195,256$

flamb(1)ant, ante $65,111,142,195$, 241

flamb(l)ant (tout neu(f)) 65, 111, 195, 257, 266

flamb(l)ant (tout $\sim \mathrm{nu}(\mathrm{d})) 65,111$, 195,257

flandrin 26, 64, 195, 261

flâner 64, 196, 261

flasque «flaque» 62,196

flasque adj. et nom $62,110,196,261$

flasquer «avoir peur» 62, 110, 196

flasquer (fer à ) 62, 110, 196

foin 64

foin sauvage 246

folle avoine 247

fondeur de cloches (sot comme un $~$ ) 245

forfaite (à $\sim) 253$

fort comme un Turc 264

fort sur la blonde 48,164

fou comme braque 255

fouillouses 250

four (cuire au de qqn) 258

four (en falloir autant que de pelotes de neige pour chauffer un ) 260

fourgonner 254

fourneau 247

fourré (sous plated) 81, 240

frais (porc ) 264

françois (soulier ) (sous soulier) 86, $121,225,250,261$

frasil 249

fréde adj. fém. 63, 135, 196, 249

fréquenter la créature 54,182

fréte adj. et nom $63,135,140,196$, 249 fréte comme glace (sous fréte, fréde) 63, 196

fréte (geler de ) 63

frette 262

fricasser 64,197

fricasser une dégelée, une ramasse (sous fricasser) 64, 197

fricasser (se de qqch.) 64, 197

frimat 249

frime (faire de rien) 256

fripper $66,197,257$

fripponner (sous fripper) 66; 197

froiser qqn 250

frôler (se contre qqn) 255

fumer «corner, biner» 245,262

fumer une touche (sous touche) 90 , $123,228,264$

fusil sans plaque (partir comme un $\sim$ ) 252

gâ ((petit) ) 23, 25, 66, 112, 133, 197

gaband 258

gaffe 256

gaffer qqn 256

gale (qui a la $\sim$ la gratte, qui ne l'a pas l'atrappe) 265

galeux comme un Écossois 60, 186

galimafrée 251

galipote (courir la ) 67, 197, 263

gallop (courir le $\sim) 252$

game(-coq) 69, 238, 251

garde-corps 67, 198, 241, 248

garder un chien de sa chienne 258

gaspiller 26, 68, 198, 264; (sous butin) 44,101

gat (petit ) "gars» 258

gavache n. masc. et fém. $67,112,198$, 262

geindre 256

geler de fréte (sous fréte) 63

Gens de terres 247 
georges (faire ses ) 68, 113, 142, 198, 264

géraniome (sous gérémium) 67

géranium (sous gérémium) 67

gérémium 67, 134, 142, 198, 250

giboulée (de neige) 27, 69, 112

ginseng (tomber comme le ) 23, 69, $112,198,265$

glace (fréte comme ) (sous fréte, fréde) 63,196

glouton 246

glumer $68,199,251$

go (tout de ) 264

go-et-gon (à ) 245

gober des mouches 265

gogo (à ) 264

goret 257

gouette n. fém. «goitre» $25,67,138$, $139,147,199,248,261$

gouliâ $23,68,113,133,199,264$

gouliafre (sous gouliâ) $68,113,199$

gourdin 250

graffigner $26,68,199,264$

graisse 69,199

graisser 69,200

graissoux, ouse adj. et nom 67,150 , 200,251

grand'côte (sous côte) 53, 104

gras comme un voleur 254

gratter 256

grelotter 263

griche-poil 69, 200, 265

griche-poil (à $\sim$ ) (sous regriche-poit) 85,220

gricher les (des) dents 69, 200

grichou 69, 200, 265

grichou (laid comme un ) 69, 200

grichou (malin comme un ) 69, 200

grincher (faire $\sim$ les dents) 253 griser (se ) 263

grossier comme un pain d'orge 262

grouiller 249

guedou (baiser en ) 67; (sous baiser) 47,159

guenille (sous amarrer) 40; (sous apichimon) 42; 201

guêpes (manger des ) 245, 259

gueusaille 19, 113, 201; (sous gueusasse) 67

gueusasse 19, 67, 113, 201, 261

gueux (la race des $\sim$ ne se perdra pas il n'y a pas mortalité de canaille) 260

guide 247,248

guides $27,67,112,248,251,259$;

(sous cordeaux) 49, 105, 180

guimbarde 27 (sous trompe) 88 ,

gum 247

habitant, ante adj. et nom 69,113 , 201, 261; (sous âbre) 41; (sous allumer) 41,99; (sous apichimon) 42, 100; (sous berline, bordel) 43; (sous bouquin) 44; (sous charger) 50; (sous équilibre) 60; (sous bouiller) 70; (sous mâle) 74 ; (sous pesâ) 79; (sous pipe) 79, 119; (sous reintier) 84; (sous soulier) 86; (sous suspect) 86; (sous traîne) 87; (sous tauraille) 88; (sous vulgaire) 90

hardes (sous butin) 44, 101; (sous gaspiller) $68 ; 201$

hardi donc «courage» 256

hausse $70,113,238,249$; (sous mitasse) 116

haut comme un chou 252

haut mal (sous mal) 75, 204, 205, 251

herbe à dinde $70,202,246,257$

herbe à la puce $70,202,246,257$

hérisson (sous port-épic) 80, 216

homme du Nord 264 
houillé [ouillé] «fatigué de qqn, de qqch." (sous bouiller) 70, 113, 202

houiller [ouiller] (se $\sim$ ) «se gorger de vin» $70,113,202$

houiller *changer, troquer» 70,113 , 202, 249

hyvernement 247

icit 71, 140, 203

immatériel, elle 71, 114, 238

impotheques 253

impropre 71

improuver 28, 70, 114, 238, 249, 259

incendie n. fém. 23, 70, 147, 203, 248

incliner 248

inconsistant, ante $71,114,239$

indian path 247

indictement 251

indicter 251

induqué 253

ingérer (s' de qqch.) 258

inquilibre (être dans l' ) (sous équilibre) $60 ; 148,203,261$

interrompre (sans qqn) 257

Jacques Cartier (curieux comme la chèvre à ) 257

jambes (avoir les $\sim$ comme des batons de traine) 253

jeu de quilles (reçu comme un chien dans un ) 260

joie (un vive la $\sim$ ) 263

joli-cour (s'appeler, se nommer ) 28 , $71,115,203,265$

jouqué 251

jouqué comme un dinde 253

jouquer (se ) 71, 115, 203

jouquoir 71, 115, 203

jour de mitasse 255

Juifrèsse $71,114,203,261$

Juive 250 jument 70, 114, 204, 249, 266

laboureur (sous habitant) 69, 113

laid comme un grichou (sous grichou) 69,200

laine (se laisser manger la $\sim$ sur le dos) 265

lait «rhum» 247

lait (petit ) «babeurre» 265

lambin 261

lambiner 261

lamper 256

langue (une $\sim$ ̀̀ percer quatre $\mathrm{mu}$ railles) 256

lard (le sera à bon marché, les cochons s'embrassent) 245

larme (une $\sim$ ) «filet» 260

lèche-crachats (sous payer) 78; 204

légerte 72, 115, 141, 204, 257

leste comme un chien de plomb 245 , 261

lever le cul comme une bête puante 252

lever (se $\sim$ le derrière le premier) 254

lévier $26,72,145,204,250$

liaison en $\mathrm{z}$ (verbe à l'impératif + pronom $+z+$ en ou y) 251

liard 246, 247

lichefrite 72, 115, 136, 204

licher 71, 115, 136, 204, 249

licher (en (à qqn)) 71, 115, 204

licher (s'en les barbes) 71, 115, 204

linge (blême comme un ) 261

livre «monnaie de compte» (sous coppre) 54

long comme d'icite à demain 253

louche adj. (sous bicleux) 45, 102; (sous loucheur) 115; (sous virel'œil) $124 ; 204$

loucher (sous bicler) 45,$102 ; 249$ 
loucheur, euse adj. et nom 27,72 , $115,204,241,242$; (sous bicleux) 102

loup-cervier 246

lundi (qui a fait $\sim$, a fait mardi) 261

lurette (belle $\sim) 254$

lynx 246

main (mal à ) 253

mains (les $\sim$ dans ses poches) 256

mains (se beurrer les $\sim$ ) (sous beurrer) $48,103,163$

maïs (sous épiochon) 59

mais c'est que 261

major, ore 75, 239

majore (tierce ) (sous major) 75, 239

mal a drette (un ) 257

mal à main 253

mal caduc 75, 205

mal de raquette 247

mal de saint $75,205,251$

mal de saint Jean 75, 205, 251

mal (être amanché) 263

mal (haut $\sim) 75,205,251$

mal (le $\sim$ passe net comme sur la main) 255

mal (se trouver ) 261

mal (tomber d'un ) 75, 204, 251

malade comme un chien 256

mâle $74,116,205,249$; (sous tuque) 88,122

malin, igne $73,205,250,262$; (sous rustique) 84

malin comme sept fois le diable 255

malin comme un Basque 46, 160

malin comme un Cree (Cris) 52, 103, 183

malin comme un grichou 69

malin (être un Basque) 46, 160, 251

mamelle (enfant à la $\sim$ ) (sous bèr) 45

manche (branler dans le $\sim$ ) 245 manche de balai (sec comme un ) 264

manche de pipe 73, 205; (sous bouquin) 44

manche d'une poêle 73,248

manchon(n)ier 26, 75, 205

manger à la croc au sel 245

manger à se mettre le ventre sur le dos 258

manger (avoir (bien) des croûtes à ) 245,265

manger de l'avoine 245, 259, 263

manger des guêpes 245, 259

manger qqn 255

manquer «faillir» 75, 206

manquer $(\mathrm{la} \sim$ belle) 75, 205

marcher après qqn 252

marcher comme un chien qui vient des vêpres 252

mardi (qui a fait lundi, a fait $\sim$ ) 261

marier «épouser» $19,20,73,116$, 206, 249, 259

marinade (sous marinage) $73 ; 206$

marinage $73,148,206,251$

maringouin 246

marmite (faire bouillir la ) 258

marri "faché» 260

martre (prendre $\sim$ pour renard) 245

maskinongé $16,75,233,248$

masquinongé 233, 246, 247

matelat $74,207,249$

matelot «insecte» $72,207,246,249$

matériel, elle 75, 239, 250

mâtin 263

matois (fin ) 262

mauvaiseté 75, 207

mauve des jardins (sous passe-rose) 81 , $212 ; 246$

méchant 73, 207, 253; (sous apichimon) 42, 100; (sous rustique) 84 
mèche "grande distance" 74,116 , 207,249 ; (sous pipe) 79, 119

mèche "extrémité d'un fouet" 74 , $116,207,242,249$

mècher $26,27,74,116,207$; (sous moucher) 74

même (à ) (sous à même) 42; 153

menoirs 28, 74, 207, 248; (sous brancard) 44 ; (sous travail) 88

mentir comme un arracheur de dents 260

mer (si la bouilloit) 258

mère orignal (sous orignal) 118

meuble «récipient» (sous casseau) 52; (sous plated) $81 ; 208$

micmac 73, 208, 250

micoine $16,73,116,234,250$

micouène 116,234

mieux (faire qqch. comme un des $\sim$ ) 263

mil 251

mine (avoir une $\sim$ à cracher dessus) 254

minot (sous empocheter) 61

mirer (se ) 262

mirlifichures «colifichets" 255

mistimus ((en) ) 23, 75, 117, 208

mitaine (sous manchonier) $75 ; 208$, 249

mitaine (être comme une $\sim$ sans pouce) 260

mitaines (le vent se tourne du côté des ) 245

mitasse 16, 74, 116, 234, 249; (sous hausse) 70,113

mitasse (jour de ) 255

miton mitaine (onguent -) 264

mitonner qqn «douilleter» 250,260

moindrement (le, la $~ 22,72,75$, $154,208,261$

moins (ni plus, ni ) 258 mondaine (orge ) $28,73,116,145$, 208

monsieur (faire le $\sim$ ) 264

montagne (là-bas sur la ) 259

Montréaliste 75, 150, 209

mortifier (faire ) 263

moucher 26, 27, 74, 209

mouches (gober des $\sim$ ) 265

mouches (on n'attire pas les $\sim$ avec du fiel) 262

mouiller «pleuvoir» $27,72,115,209$, $249,260,266$

mouillette 249

mouillette (avoir un oeil à la coque et l'autre à la ) 245

moule (fait au ) «au tour» 254

moulin (chacun à son tour comme au ) 254

moulin de La Chine (être comme le ) 245

moustic 246

moutarde (prendre au nez comme la ) 256

moyennement 255

mucre 258

mulon de foin 73, 209, 251; (sous foin) 64 ; (sous veuilloche) 91

mur (à moi le ) 245

nager à grande eau 256

nation sauvage (sous Cree) 103

naturel, elle 76, 117, 209

naturellement 263

nége (sous abât) 100; (sous bordée) 102 ; (sous giboulée) 112; 134

neige (pelote de $\sim$ ) (sous pelote) 80, 212

ni quoi, ni qu'est-ce 245,264

nijon 27, 76, 117, 209, 262; (sous bougon) 48

niveleux, euse 76, 210, 250

nix (for stein) 76, 210, 251 
nix-nix 76

noix amère, douce 264

nord (homme du ) 264

nord-est (sec comme ) 264

notureau 76, 141, 210, 249; (sous porchais) 80

obligation 248

obligé, ée (être pour) 78, 239, 248, 259

œil (avoir un à la coque et l'autre à la mouillette) 245

office $20,77,118,210,239,248$

oiseau (comme l' sur la branche) 257

oiseau blanc $26,78,211,246,247$

onglée 249,262

onguent miton mitaine 264

opportunité 248

ordonné, ée (être de) 78, 117, 239, 251

oreille (entrer par une $\sim$, sortir par l'autre) 261

oreille de soulier 77

orge mondaine (sous mondaine) 73 , 116,145

orge mondé 249

orges (faire ses $\sim$ ) (sous georges) 68, 113

originer $20,78,117,239,251$

orignal $23,77,118,211,246,249$

orignal (mère ) 118

ortolan (sous oiseau blanc) 26, 78; 211

ostiné 254

o'tic «caribou» 246, 247

otocas $16,78,137,235,246$

oubli 250

oublie 254

ouète $24,77,138,211$

ouette 250

ouiller (v. houiller) paille 250

pain d'épice (sec comme un ) 264

pain d'orge (grossier comme un $\sim$ ) 262

pain (perdre un sur sa fournée) 245, 263

paire (une $\sim$ de soufflets) 256

palette 80

pantère 246, 247

papier brouillard 249

papier cassé 249

papier-nouvelle 80, 211, 250

papier-public (sous papier-nouvelle) 80,211

papier tenture 250

paradis (arbre du ) (sous épinette) 62, 189,246

paré, ée 78, 118, 211, 248, 262

paresse (relever du péché de ) 262

parler comme la Bouche 260

parler comme saint Paul 260

parler comme une femme 256

parler du nez 261

parler françois à qqn 256

paroli (avoir un beau ) 253

partir comme un fusil sans plaque 252

passe-rose 26, 81, 212, 246

passer en belette 256

patira (en faire un ) 252

Paul (parler comme saint ) 260

pauvre comme un rat d'église 260

pauvreté (tomber comme la $\sim$ sur le monde) 256

payer chopine (sous bouiller) 113

payer ses complimens, ses respects, une visite $20,27,28,78,118$, $240,248,260$

paysan (sous habitant) 113, 261

peau (devoir plus à sa qu'à sa chemise) 254 
pêcher «trouver» 258

peigné (un mal ) 263

peinture $(\mathrm{en} \sim) 254$

peinturer (sous cariole) 50; 212

pelle (renvoyer qqn la $\sim$ au etc.) 263

pelleter $81,212,242,251$

pelote «balle»; «balle du jeu de paume» 80, 212, 249; (sous retontir) 84

pelote de neige 80,212

pelotes de neige (en falloir autant que de $\sim$ pour chauffer un four) 260

pendu (avoir de la corde de $~ 263$

pendu (sec comme un ) 265

perdre un pain sur sa fournée 245 , 263

perdrix 81, 213, 246, 247

perdu (chanter comme un ) 257

pesâ 79, 133, 213, 249, 260

pesant n. masc. $81,213,263$

pésant, ante adj. (sous berline) $43 ; 134$

peter $23,27,80,119,214,242$; (sous retontir) 84

pièces 247

pieds (avoir les quatre blancs) «être libre» 256

pieds (les lui brûlent) 245

pierre à calumet 246, 247

piéter (se ) 253

piler sur qqn 255

pincettes (baiser en $\sim$ ) «tromper» (sous baiser) 47, 159; (sous pincettes) $81 ; 259$

pincettes (baiser en $\sim$ ) «embrasser» (sous baiser) $47 ; 214$

pipe (manche de $\sim$ ) (sous manche) 73 , 205 ; (sous bouquin) 44

pipe "mesure de distance» 26, 79, 119, 214, 249; (sous mèche) 116

pique-bois 258

piquet (droit comme un ) 263

\section{Piroli 257}

pistolage «sorte de limaçon» 80,214 pivac «bivouac» 258

place (dans la ) (sous écrapoutir) 61; 214,264

plaindre (se $\sim$ le ventre plein) 256

plairie 28, 79, 142, 215, 249

plaisant, ante $19,81,119,215$

plancher des vaches 91,265

plat (être amis jusqu'au ) 256

plated 81, 240, 251

plein (couteau $\sim$ de graisse) 254

plein ((fin) tout $\sim) 251,254$

pluie (faire la $\sim$ et le beau temps) 257

plus (ni $\sim$, ni moins) «cependant» 258

poche (sous pochetée) $81 ; 215,265$

poche (connaître qqch. comme sa $\sim$ ) 263

pochetée 81,215

poison n. fém. 254

poisson blanc $81,216,246$

poisson doré $81,216,246,247$

porcelaine (sous pistolage, pucelage) 80 porcelaine (sous porceline, pourceline) 80

porceline $80,136,216$

porc-épic 80,216

porc frais 264

porchais $80,216,250$; (sous notureau) 76

portage 247

port-épic 80, 145, 216

portépis 251

porter (ne pas à terre) 253

poste à Saupin 260

postillon (sous verbalement) 91, 124

pou (être comme un sur une gale) 264

pouce (argent sous le $\sim) 255$ 
pouce (être comme baise mon ) 252 pouce (être comme une mitaine sans ) 260

poudine $79,119,147,216$

pouding (sous poudine, poutine) 79 , 119

poudrer $18,79,217$

poudrerie $18,79,217,249,259$

poulailler 253

pouliche 250

pourceline $80,136,137,217,250$

poutine $79,119,217$

praline 247

prendre une belle embardée 255

presque «preste» 253

presquement «prestement» 253

preuve (à ) 245

procès-verbal (avoir la tête comme un ) 245

propre comme un sou 256

prouvable $28,80,119,217,250,262$

pucelage «sorte de limaçon» 80,218 , 250

quatre à quatre (descendre les escaliers ) 263

Québecquois 82,218

Québéquois 82, 218

quelouer «clouer» 258

quêter $82,120,218$

quêteur, euse $25,82,120,218,251$

quêteux 254

queue (bête à la grande ) 256

quitte (avoir plus de $~$ ) 23, 82, 120 , 219

quitter 82, 219

rabougris 255

racéré, ée 83

racérer $26,83,219$

racopié (son père tout $\sim$ ) 252 rafistoler 261

raison 253

raisons (donner de mauvaises $\sim$ ) 253

râle 251

râler 251

ramancher «conter, raconter des choses insensées». 19, 82, 120, 219,248

ramancher "raccommoder, réparer" 82, 120, 219, 248, 259; (sous amancher) 40,99

ramasse 84,219

ramasse (donner une ) 84; (sous dégelée) 56

ramasse (fricasser une $\sim$ ) (sous fricasser) $64,197,262$

ramer sans culottes 245

rapapillotter 261

rapide 247

rapiéceter 152,220

rapiesté, ée $85,121,257$

rapiester $85,121,152,220$

raquette (sous pelote) 80,$213 ; 249$

raquette (mal de $\sim) 247$

rat d'église (pauvre comme un 260

ratapiat $84,220,263$

raz (à terre) 265

raz (tout à $\sim) 265$

razer 265

(re)brousse-poil (à ) 85, 220

refection (content a $\sim) 256$

régal 253

régaler (se ) 253

regarder par-dessus l'épaule 264

regriche-poil (à ) 85, 220

regricher 85,220

reintier $84,121,221$

relevée $82,120,221,248$

remercier pour 84, 259; (sous obligé) 78 
remettre 250

remettre sur farine 245

remou(s) 83

remue-ménage 250

renâcler 262

renard (prendre martre pour ) 245

renchausser 255

rênes (sous cordeaux) 27, 49, 105, 180, 251; (sous guides) 67, 112

renflé comme une souppe au lait 260 renne (sous caribou) 50, 246

renoter 254

rente de l'argent «intérêt» 255

requise (bois de ) 253

résous $84,121,153,221,249$

respect (sur votre $\sim$ ) 265

ressoudre 258

retailles 251

retaper qqn (sous baiser) 47; (sous guedou) 67; (sous pincettes) 81; 221, 258

retentir (sous retontir) 84

retontir "rebondir" $26,84,221$

retontir "retentir» 84, 222, 251

retraire (sous retraiter, retrayer) 83, $121 ; 222$

retraiter «battre en retraite» 83,120 , 240, 249

retraiter "retirer» $83,120,222,249$

retrayer "retirer" $83,121,153,222$, 249

rêve (chanter comme un $) 252$

reveil matin 257

revirer (se ) 254

rhimb de vent 85,222

ribote (faire ) 264

ricaner 257

ric-à-rac 245

rigole 249 rime (n'avoir pas de ) 257

rin de vent 257

rire jaune 253

risée de vent 257

riz sauvage 247

robe de bœuf $82,222,249$

Roger bon temps 263

rôle $27,83,240$

rose-d'outremer (sous passe-rose) 26, 81,212

rose-tremière (sous passe-rose) 26,81 , 212

roue (être comme une troisième $\sim$ à une charrette) 260

roulin (avoir du ) 254

roustaut 263

rubrique 251

rustique 84, 223, 250, 262

sable (jeter du dans les yeux) 265

sacacoua 247

sagamité 252

saint épais (un ) 262

salopries «saletés, ordures» 256

saoul 257

saoul (boire, jouer, manger son ) 256

sarpidon 86, 122,134, 150, 223

sas (couler comme un ) 254

sas (passer au gros ) 254

sasser 254

Saupin (poste à ) 260

sauvage adj. et nom (sous apichimon) 100; (sous boucaner) 101; (sous hausse) 70, 113; (sous micoine) 73, 116; (sous mitasse) 116; (sous traine de clisse) 88; 223

sauvage n. fém. (sous sauvagesse) 87 , 122,224

sauvage (nation ) (sous Cree) 103

sauvage (riz ) 247 
sauvage (soulier $\sim$ ) (sous soulier) 86, $121,225,261$

sauvagesse $87,122,223$

savant comme un livre 260

savon (brique de ) 255, 265

savon (donner un ) 262

savonner «réprimander» (sous brâsser) 46,$102 ; 224$

sciau 261

scieau 249

scier les bleds (sous couper) $51 ; 224$

sec comme nord-est 264

sec comme un coton de bled d'Inde (sous coton) 54, 264

sec comme un écopeau (sous écopeau) 59,186

sec comme un manche de balai 264

sec comme un pain d'épice 264

sec comme un pendu 265

secquement 256

semer qqn 258

sentinelle n. masc. 85, 147, 224, 248

servante du curé (n'être pas la ) 245

seul (tout fin ) 257

sieau $27,86,122,136,224$

sifflet (couper le $~ 256$

soc (sous reintier) 84,$121 ; 224$

soc de porc frais 264

soigner 86

sol «monnaie» (sous voyage) 90, 263

sols (faire de cinq six blancs) 265

son (farine du diable retourne en $\sim$ ) 262

sorcière $86,224,249$

Sorel «habitant de Sorel» 87, 224

sortir d'entrer 259

sortir dehors 259

sot comme un fondeur de cloches 245 sou «monnaie de billion» (sous coppre) 54

sou (propre comme un ) 256

soulier de bœuf $86,121,225,250$, 261

soulier françois $86,121,225,250,261$

soulier sauvage $86,121,225,261$

soupe (dormir comme une $\sim$ ) 87, 225

soupe (trempe comme une $\sim$ ) (sous trempe) $89,123,230,260$

soupirail 257

souppe au lait (renflé comme une $\sim$ ) 260

souppe (docteur en salée) 257

souppe (tremper la $\sim) 260$

sourd (crier comme un ) 257

souris-chaude $86,225,246$

stellà $86,151,225$

stellci(t) $86,151,225$

stroud blanket 247

style (dans le grand, le haut ) 27, 85, 240, 249

sucet $26,87,226$

suerie 245

superbe 250

sur «chez" $86,122,155,226$

sur, ure $87,122,261$

sur (pour le $\sim$ ) «certain» 258

surir $87,122,251$

sus «chez» 155,262

suspect, ecte $86,122,226,250$

tabac du diable «jusquiame» 265

tabaconiste 265

tabagiste 265

tambour 249

tanner qqu 259

tantòt 255

tapin $89,123,226$ 
tapin (flanquer un $\sim$ par les babines) 260

tapisserie 89, 250

tapon $89,123,226,251$

tarabusquer 258

tasse (boire à la grande ) 256

tata (sur un ) 258

tauraille $88,227,249$

taure (sous tauraille) $88 ; 227,249$

taurmenter (sous étriver) 60, 191

taverne (sous cantine) 53, 104, 176

tavernier, ière (sous cantinier) 53, 104, 105, 176

temps à ne pas mettre les chiens dehors 261

tenseulement 263

terre (coup de $\sim$ ) (sous retontir) 84

terre (ne pas porter à $~ 253$

terres (Gens de ) 247

tête (avoir la $\sim$ comme un procèsverbal) 245

tête d'Allemand (avoir, être une ) 42, 157,261

tête de boule $90,227,247,265$

tête d'escargot 90, 190, 265

tête (ne pas avoir la $\sim$ aux pieds) 245

thétière 88, 143, 227

tierce majore (sous major) 75, 239

tigre 246, 247

tinter (sous retontir) 84 ; (sous tinton) 90

tinton $23,90,124,227,251,265$

tiran de soulier 77

tirans $89,227,249$; (sous clairon) 54

tire $18,27,89,227,249$

tisons (cracher sur les ) 245

tomber comme la pauvreté sur le monde 256

tomber comme le ginseng (sous ginseng) $23,69,112,198,265$ tomber comme une tourte 257

tomber d'un mal (sous $m a l$ ) 75, 204, 251

tondre n. masc. «amadou» 88,147 , $228,246,249$

touche (fumer une ) $90,123,228$, 264

tour (chacun à son comme au moulin) 254

tournailler qqn 253

tourniquet 90,228

tourte (tomber comme une $\sim$ ) 257

tourte (tuer roide comme une $\sim$ ) 257

tout de bon 253

train «occupations quotidiennes du ménage» 89, 123, 228

train «tapage» 259, 261

train (en ) «légèrement ivre» 89,123 , $229,242,250$

traîne $87,229,248,259$; (sous travail) 88

traîne de clisse 88, 229, 248, 264

traîneau $27,88,229,248$; (sous cariole) 50 ; (sous traine) 87

travail 28, 74, 88, 230, 248, 259; (sous brancard) 44

trèfe $89,139,230$

trèfle 250

trembler (faire ) 245, 250, 254

trembler les fièvres 262

trempe ((tout) ) 26, 89, 123, 230, 260

trempe comme une soupe 89,123 , 230,260

tremper la soupe 260

tricherie (la $\sim$ retourne à son maître) 262

tricoler $89,123,230,250$

tripe de roche 247

tripoter 264

troisse $23,89,140,230,250$ 
trompe $27,88,230,248$

trou (boire comme un ) 260

trouver (se mal) 261

tuer «éteindre» 87, 122, 231, 248, 259

tuer roide comme une tourte 257

tuque $18,88,122,231,249,261$;

(sous mâle) 74, 116

Turc (fort comme un ) 264

urson (sous port-épic) 80, 216

vaches (plancher des $\sim$ ) 91, 265

vaisseau «navire» (sous envarié, ée) 58

vaisseau "récipient" 122

valeur (de ) 245, 250, 260

valoir (ne pas $\sim$ les quatre fers d'un chien) 261

valoir (ne pas son plein cul d'eau chaude) 265

valtreux (être un ) 252

varengues 247

veau (brailler comme un ) 252

vent (le se tourne du côté des mitaines) 245

vent (rhimb de $\sim$ ) (sous rbimb) 85 , 222

vent (rin de $\sim$ ) 257

vent (risée de ) 257

vêpres (marcher comme un chien qui vient des ) 252

vêpres (reçu comme un chien à ) 260

verbalement $91,124,231,251,255$ verglas 250

veuilloche $91,231,251$; (sous foin) 64

vie (arbre de $~$ ) (sous épinette) 62, 189,246

vire-l'œil $27,90,124,231,250,264$;

(sous bicleux) 102

vire-main (dans un ) 256

vite pendant que c'est chaud witement» 253

vive (un la joie) 263

vocation 253

voie (sous voyage) 90, 124; 231

voir (voyons 260

voleur (gras comme un $\sim$ ) 254

vomir tripes et boyaux 261

vouloir que (je voudrois que cela seroit) 259

voyage (d'eau) $90,124,232,242,249$, 261

voyageur (sous jument) $70 ; 232,247$, 248

vulgaire $23,90,124,232,250,262$

waac «tripe de roche» 247

warrant 251

washes 247

wattap 247

yeux (avoir les $\sim$ à la perdition de son âme) 253

yeux (jeter du sable dans les $\sim$ ) 265 